\title{
RBU: A COMBINED MONTE CARLO REACTOR-BURNUP PROGRAM FOR THE IBM 709
}

\author{
ATL Job C-13 \\ By \\ E. J. Leshan \\ J. R. Burr \\ M. Temme \\ G. T. Thompson \\ J. R. Triplett
}

September 30,1959

A Division of American-Standard Advanced Technology Laboratories Mountain View, California

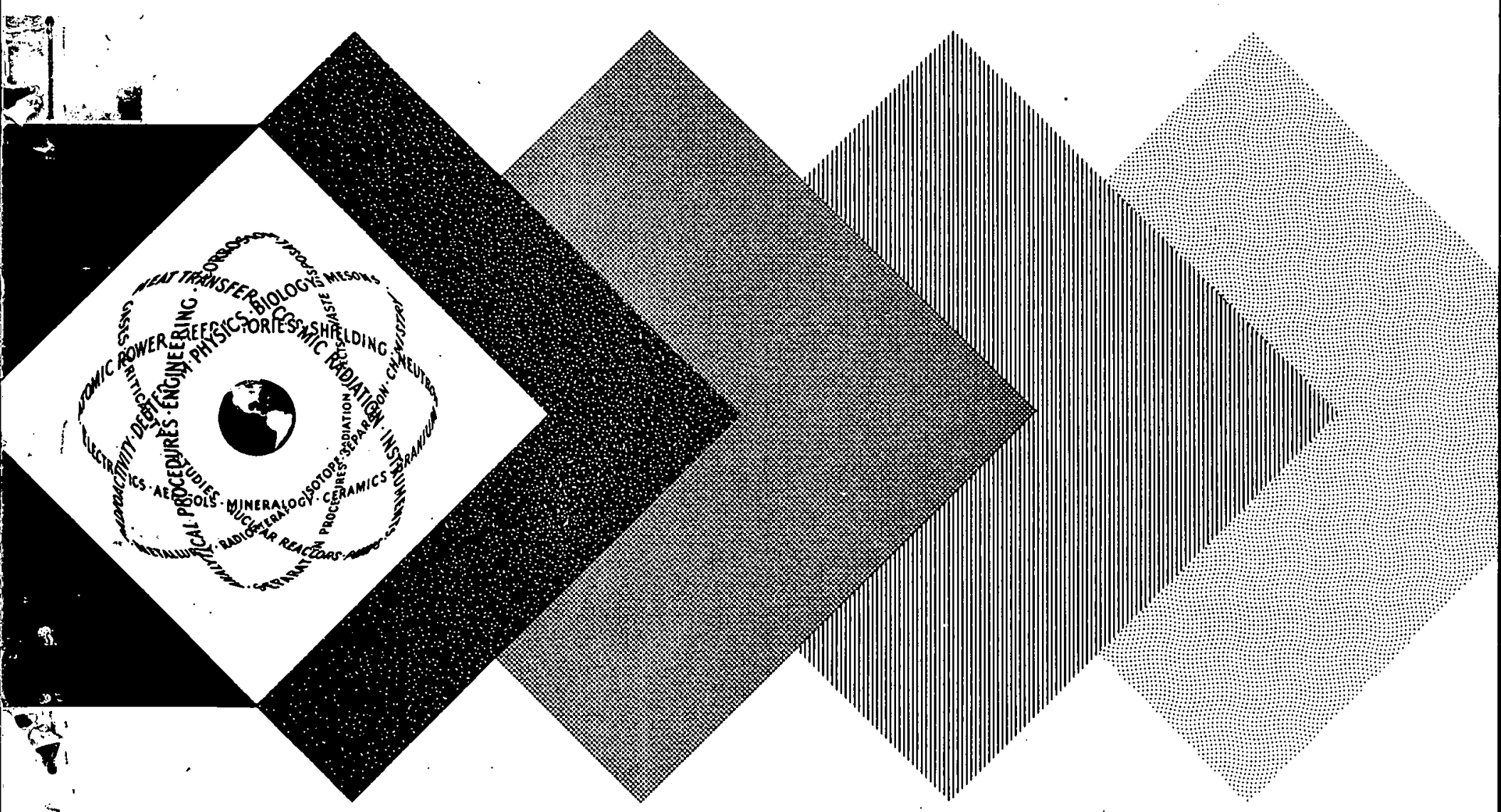




\section{DISCLAIMER}

This report was prepared as an account of work sponsored by an agency of the United States Government. Neither the United States Government nor any agency Thereof, nor any of their employees, makes any warranty, express or implied, or assumes any legal liability or responsibility for the accuracy, completeness, or usefulness of any information, apparatus, product, or process disclosed, or represents that its use would not infringe privately owned rights. Reference herein to any specific commercial product, process, or service by trade name, trademark, manufacturer, or otherwise does not necessarily constitute or imply its endorsement, recommendation, or favoring by the United States Government or any agency thereof. The views and opinions of authors expressed herein do not necessarily state or reflect those of the United States Government or any agency thereof. 


\section{DISCLAIMER}

Portions of this document may be illegible in electronic image products. Images are produced from the best available original document. 


\section{LEGAL NOTICE}

This report was prepared as an account of Government sponsored work. Nelther the United States, nor the Commission, nor any person acting on behalf of the Commiselon:

A. Makes any warranty or representation, expressed or implied, with respect to the accuracy, completeness, or usefulness of the Information contalned in this report, or that the use of any information, apparatus, method, or process disclosed in this report may not infringe privately owned righte; or

B. Assumes any liabilities whth respect to the use of, or for damages resulting from the use of any information, apparatus, method, or process disclosed in thls report.

As used in the above, "person acting on behalf of the Commission" includes any employee or contractor of the Commission, or employee of such contractor, to the extent that such employee or contractor of the Commission, or employee of such contractor prepares, disseminates, or provides access to, any information pursuant to his employment or contract with the Commission, or his employment with such contractor.

This report has been reproduced directly from the best available copy.

Printed in USA. Price $\$ 4.00$. Avallable from the Office of Technical Services, Department of Commerce, Washington 25, D. C.

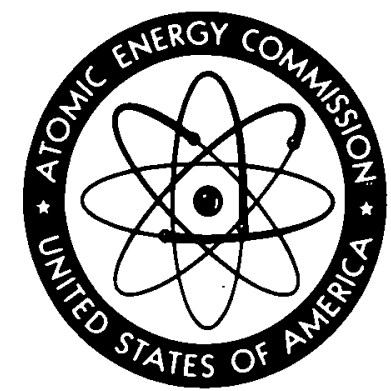


ATL-A-101

RBU: A COMBINED MONTE CARLO

REACTOR-BURNUP PROGRAM FOR THE IBM 709

ATL Job C -13

30 September 1959

$\hat{\mathrm{i}}$

$n$

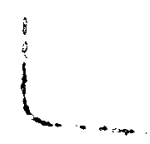

a.

E. J. Leshan

J. R. Burr

M. Temme

G. T. Thompson

ADV ANCED TECHNOLOGY

LABORATORIES
Prepared under

Contract No: AT(04-3)-109

Project Agreement K

for the U. S. Atomic Energy Commission

Division of Reactor Development

by

\author{
J.R. Triplett \\ HANFORD LABORATORIES \\ OPERATION
}

ADVANCED TECHNOLOGY LABORATORIES

A Division of American-Standard

369 Whisman Road

Mountain View, California 


\section{ACKNOWLEDGMENT}

It is a pleasure to acknowledge the technical and literary advice of a number of people who have contributed important ideas to this work. Among them are E. R. Cohen, Joel Franklin, E. G. Joki, R. E. Moore, W. H. Newkirk, R. van Norton, M. J. Nowak, S. Oleksa, C. M. Rice, R. D. Richtmyer, M. Sachs, M. Walt, and J. W. Webster. The inclusion of these names in a list of this sort cannot satisfactorily express our appreciation for the help they have given.

This work was performed under Contract No. AT(04-3)-109 with the U. S. Atomic Energy Commission under the technical guidance and supervision of John Triplett of Hanford Laboratories Operation. 
CONTENTS

\section{$\underline{\text { Section }}$}

Abstract

1

II Introduction

III Microscopic and Macroscopic Fluxes

IV General Outline of the RBU Method

V . The Monte Carlo Portion of RBU

A. Geometry

B. Description of a Material

11

C. General Outline of the Monte Carlo Portion

D. Sampling from Initial Neutron Tape

E. Unresolved Resonances

F. Resolved Resonances

G. Between Collisions Routine 18

H. Collision Routine 25

I. Census Time 32

J. Reflection at Outer Boundaries

VI Monte Carlo Constants

VII The Diffusion Calculation

A. The Difference Equations

B. Numerical Method

C. Acceleration.

D. Specific Numerical Procedure

VIII The Diffusion Constants

IX Iteration on the Reactivity

$\mathrm{X}$. The Burnup Calculation

A. The Predictor-Corrector Method

B. Changing the Time Interval 
$\begin{array}{lll}\text { XI Burnup Constants } & 62\end{array}$

XII Cross Section Preparation Routine 64

XIII Mathematical Details _ 69

A. Selection from Probability Distributions $\quad 69$

B. The Calculation of Psi and Chi $\quad \therefore \quad 72$

C. Resonance Equations $\quad 79$

D. Derivation of the Backward Difference Equations 84

E. Derivation of the Diffusion Difference Equations 85

XIV Tape Layouts $\quad 89$

A. RBU Instruction Tape, Tape $1 \quad 89$

B. Basic Library (Preliminary) 91

C. Cumulative Ċross Section Tape (Preliminary) 96

D. Tape 2 100

E. Tape $3 \quad 102$

F. Tape $4 \quad 111$

G. Tape 5 (and 7) 113

H. Tape $6 \quad 117$

I. Tape $8 \quad 121$

J. Tape $9 \quad \cdot 122$

K. RBU Output Tape, Tape 10 , 126

XV Memory Maps . 135

A. Diffusion Code 、 135

B. Burnup Code 143

C. Monte Carlo $\quad 149$

XVI List of Input Data (Tentative) $\quad 160$

A. Material and Energy Group Set Data 160

B. Geometric Information 164

C. Burnup Data $\quad$. 167

D. Diffusion Data . $\quad 167$ 
E. Criticality Data $\quad 169$

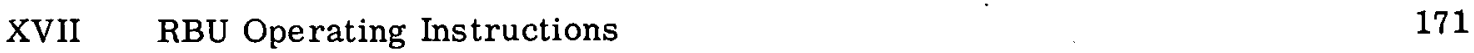

A. Making Instruction Tape 171

B. Translating Input (Input Routine) 17.2

C. Problem Execution and Restart (without changes) 172

D. Restart with Changes 173

E. 'RBU Monitor $\quad 175$

F. Tape Position and Restart Cards 178

G. Input Change Cards 179

H. Tape Position Block 180

XVIII RBU Flow Charts $\quad$. 181

RBU De-Bugging Flow Chart - 1 sheet

Overall Flow Chart - 2 sheets

Condensed Monte Carlo Flow Chart - 3 sheets

Monte Carlo - 12 sheets

Pre-Diffusion - 1 sheet

Diffusion Calculation - 6 sheets

Post Diffusion -2 sheets

Pre-Burnup - 1 sheet

Burnup - 2 sheets

Input Routine (Preliminary) - 8 sheets

Pre-Pre and Pre Monte Carlo - 12 sheets

Post Monte Carlo - 12 sheets 


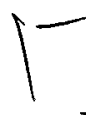

I. ABSTRACT

RBU is a digital computer program for the detailed calculation of the neutron, reactivity, and isotopic history of a reactor in which relatively exact models of the geometry and physical processes are included to permit reliable predictions of fuel costs and reactor performance. The program uses the monte carlo method to obtain the fine structure of the neutron flux in three space dimensions and energy. Using this fine structure, cross sections are averaged over space and energy to obtain the neutronic properties for equivalent homogeneous one-dimensional regions of space and ranges of energy. These are used in diffusion calculations to obtain the macroscopic flux distribution throughout the reactor. The consumption and production of isotopes is computed for a time step by the solution of sets of partial differential equations involving both the macroscopic and microscopic fluxes. With the new concentrations, diffusion calculations are performed again to obtain macroscopic fluxes for the next time step. At variable intervals, monte carlo calculations are again performed to determine the changes in microscopic flux distributions. The cycle is repeated until conditions on the reactivity or other properties dictate the end of the calculation. Programmed control rod manipulation may be included in the calculation. The monte carlo, diffusion, or burnup portions of the program may be used separately.

. The unresolved resonance range is treated by random selection of resonance parameters from appropriate distributions using the Doppler broadened single level Breit-Wigner formula. Resolved resonances are treated similarly with the exception that specific values of the resonance parameters are used. The effects of molecular binding and thermal motion of the nuclei on near-thermal scattering are treated by a simple model capable of incorporating the pertinent physical theory and data. 


\section{INTRODUCTION}

The purpose of this report is to describe a method of predicting the reactivity and the distribution of isotopes in a nuclear power reactor as functions of its operating history.

The problem has, of course, been frequently approached using approximations of varying complexity, the accuracy and applicability of which are, to some extent, uncertain. In the method presented in this report, an attempt is made to eliminate many of the uncertain approximations in order to increase the range of applicability of the calculations. A digital computer program, RBU, using this method and developed for the IBM 709 by Advanced Technology Laboratories (ATL), a Division of American Radiator \& Standard Sanitary Corporation, under contract to the Hanford Laboratories, performs the calculations described.

In this report, a statement of the problem will be given first; second; the concepts of microscopic and macroscopic flux are mathematically described; third, the method of approach is outlined; fourth, various sections are presented which describe the monte carlo portion of the code, the diffusion calculation, the burnup calculation, and the preparation of the various constants and cross sections needed in the program. Finally the details of the use and structure of the machine code are given.

Essentially the problem is one of determining the spatial and energy distribution of the neutron flux; the reactivity at a given time may be computed from the integrals over volume and energy of the products of various nuclear constants with the flux and from the integral over the outer surface of the reactor of the normal component of the flux. Similarly, the rate at which the various isotopes are consumed and produced at each point in the reactor may be computed from the integral over energy of the product of neutron flux and the cross sections for the relevant processes and from the decay constants for those nuclides which are radioactive. The determination of the neutron flux is complicated by the rapid variation of some cross sections with energy, of some concentrations with position in the reactor, and by the three-dimensional structure of the system. Consequently, a very fine coordinate spacing is required for a reliable solution until sufficient experience 
has been acquired to permit generally valid approximations to be made. For this reason, and because of the complex geometry involved in some fuel elements and control systems of interest, the monte carlo method is used in RBU for some of the neutron flux calculations. The program then uses the monte carlo results to obtain constants with which most of the flux determinations can be made by simpler methods. The neutron flux distribution, as a function of time in a reactor, is also effected by the movements of control rods used to maintain criticality and to flatten flux. In RBU, this control rod motion is closely simulated by changes in the nuclear constants of special regions in a sequence which is determined by input specifications and, to an extent, determined by computed reactivity. 


\section{MICROSCOPIC AND MACROSCOPIC FLUXES}

In the following section, the theoretical basis for the process of averaging over heterogeneities and of forming a one-space dimensional model for a three dimensional system is briefly discussed.

The neutron transport equation

$$
\begin{aligned}
\frac{1}{v} \frac{\partial \phi(\vec{r}, \vec{v})}{\partial t} & =\frac{1}{v} \vec{v} \cdot \vec{\nabla} \phi(\vec{r}, \vec{v})-\Sigma_{t}(\vec{r}, v) \phi(\vec{r}, \vec{v}) \\
& +\int \overrightarrow{d v}^{\prime}\left[\frac{1}{4 \pi} v_{f}\left(\vec{r}, v^{\prime}\right) \Sigma_{f}\left(\vec{r}, v^{\prime}\right) x_{f}(v)\right. \\
& \left.+\frac{1}{4 \pi} \nu_{\text {in }}\left(\vec{r}, v^{\prime}\right) \Sigma_{\text {in }}\left(\vec{r}, v^{\prime} \rightarrow v\right)+\Sigma_{s}\left(\vec{r}, \vec{v}^{\prime} \rightarrow \vec{v}\right)\right] \phi\left(\vec{r}, \vec{v}^{\prime}\right)
\end{aligned}
$$

express the rate at which the density of neutrons increases at each point in phase space in terms of the neutron flux density, its gradient and the various nuclear cross sections. If the region between $(\vec{r}, v, \vec{\Omega})$ and $(\vec{r}+\Delta \vec{r}, v+\Delta v, \vec{\Omega})$ is donoted as cell $i$, Equation (1) may be integrated over the cell as follows:

$$
\int \mathrm{dvdr} \frac{1}{\mathrm{v}} \frac{\partial \phi}{\partial \mathrm{t}}=-\int_{\mathrm{i}} \mathrm{dvd \vec {r }} \frac{1}{\mathrm{v}} \overrightarrow{\mathrm{v}} \cdot \vec{\nabla} \phi \ldots \text { etc. }
$$

The integrals may be replaced by the products of the volume of a cell and the average value of the integrand in the cell. The index i can be replaced by coarse variables, $\vec{R}, \vec{V}$, which are essentially identical to $\vec{r}$ and $\vec{v}$, except that they imply a macroscopic point of view. Finally, one obtains an equation identical to(1) in appearance.

$$
\begin{aligned}
\frac{1}{V} \frac{\partial \phi(\vec{R}, \vec{V})}{\partial t} & =-\frac{1}{V} \vec{v}^{\prime} \cdot \vec{\nabla} \phi(\vec{R}, \vec{V})-\Sigma_{t}(\vec{R}, V) \phi(\vec{R}, \vec{V}) \\
& +\int d \vec{V}^{\prime}\left[\frac{1}{4 \pi} \cdot \nu_{f}\left(\vec{R}, V^{\prime}\right) \Sigma_{f}\left(\vec{R}, V^{\prime}\right) x_{f}(V)\right. \\
& \left.+\frac{1}{4 \pi} \nu_{\text {in }}\left(\vec{R}, V^{\prime}\right) \Sigma_{\text {in }}\left(\vec{R}, V^{\prime} \rightarrow V^{\prime}\right)+\Sigma_{s}\left(\vec{R}, V^{\prime}, \vec{V}^{\prime}, V\right)\right] \phi\left(R, V^{\prime}\right)
\end{aligned}
$$

in which the use of the variables $\vec{R}$ and $\vec{V}$ as arguments of a function implies that it is a local average. 
Equation (3) is considerably simpler than (1) for two reasons. First, the locally averaged cross sections vary slowly with position in the reactor and with energy and.may be treated as constants over relatively large regions. Secondly, the locally averaged fluxes vary slowly with position, so that it is often satisfactory to replace (3) by the diffusion theory approximation to it.

The local averages used in Equation (3) are defined according to their use in (1)

$$
\begin{aligned}
& \left.\phi(\vec{R}, \vec{V})=\int d v \int d \vec{r} \phi \overrightarrow{(r}, \vec{v}\right) / \Delta \\
& \Sigma_{t}(\vec{R}, v)=\left[\int d v \int \overrightarrow{d r} \vec{t}_{t}(\vec{r}, v) \phi(\vec{r}, \vec{v})\right] / \phi(\vec{R}, \vec{v}) \Delta,
\end{aligned}
$$

et cetera,

where $\Delta$ represents the four dimensional cell volume.

For a generally (but not necessarily an exactly) cylindrical system, (3) may be integrated over two of the cylindrical coordinates $\theta$ and $Z$, so that an equation involving only one variable, $\mathrm{R}$, the distance from the cylindrical axis is obtained:

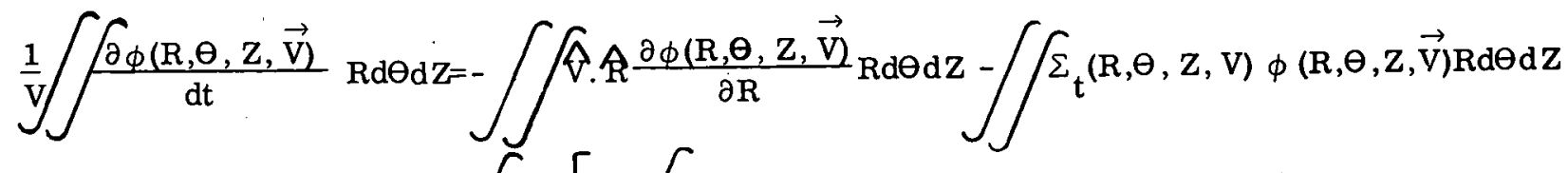

$$
\begin{aligned}
& +\int d^{\prime} \cdot\left[\frac{1}{4} \pi \int \nu_{f}\left(R, \Theta, Z, V^{\prime}\right) \Sigma_{f}\left(R, \Theta, Z, V^{\prime}\right) x_{f}(V) R d \Theta d z\right) \\
& +\frac{1}{4 \pi} \quad \nu_{\text {in }}\left(R, \theta, Z, V^{\prime}\right) \Sigma_{\text {in }}\left(R, \Theta, Z, V^{\prime} \rightarrow V\right) \operatorname{Rd} \theta d Z \\
& +\quad \Sigma_{s}\left(R, \Theta, Z, V^{\prime}, \overrightarrow{V^{\prime}} \cdot \overrightarrow{V^{\prime}}\right) \phi\left(R, \Theta, Z, V^{\prime}\right) \\
& -\hat{V} \cdot \hat{z}\left[\int \phi(R, \theta, H, V) R d \theta-\int \phi(R, \theta,-H, V) R d \theta\right] \text {, }
\end{aligned}
$$

Where $\widehat{V}, \widehat{R}$, and $\widehat{\mathrm{Z}}$ are appropriate unit vectors. 
The last term accounts for the leakage at the ends, $\mathrm{Z}= \pm \mathrm{H}$ of the reactor;

actually $\mathrm{H}$ may be a function of $\mathrm{R}$ without affecting the validity of (5).

The appearance of Equation (5) may be simplified by replacing the integrals over $\Theta$ and $\mathrm{Z}$ by quantities which have been averaged over these variables. Average quantities are indicated by underlining. On dividing by $4 \pi \mathrm{RH}$, one obtains,

$$
\begin{aligned}
\frac{1}{\mathrm{~V}} \frac{\partial \phi(\mathrm{R}, \overrightarrow{\mathrm{V}})}{\partial t} & =-\hat{\mathrm{V}} \cdot \hat{\mathrm{R}} \frac{\partial \Phi(\mathrm{R}, \overrightarrow{\mathrm{V}})}{\partial \mathrm{r}}-\underline{\Sigma}_{\mathrm{t}}(\mathrm{R}, \mathrm{V}) \Phi(\mathrm{R}, \overrightarrow{\mathrm{V}}) \\
& +\int \mathrm{d}^{\prime} \cdot\left[\frac{1}{4 \pi} \nu_{\mathrm{f}}\left(\mathrm{R}, \mathrm{V}^{\prime}\right) \underline{\Sigma}_{\mathrm{f}}\left(\mathrm{R}, \mathrm{V}^{\prime}\right) x_{\mathrm{f}}(\mathrm{V})\right. \\
& \left.+\underline{\Sigma}_{\mathrm{S}}\left(\mathrm{R}, \mathrm{V}^{\prime}, \overrightarrow{\mathrm{V}}^{\prime} \cdot \overrightarrow{\mathrm{V}}\right)\right] \Phi\left(\mathrm{R}, \overrightarrow{\mathrm{V}}^{\prime}\right)-\underline{\mathrm{L}}(\mathrm{R}, \overrightarrow{\mathrm{V})}
\end{aligned}
$$

where

$$
\begin{aligned}
& \Phi(R, \vec{V}) \equiv \frac{1}{4 \pi H} \int_{-H}^{H} d Z \int_{0}^{2 \pi} d \Theta \phi(R, \Theta, Z, \vec{V})
\end{aligned}
$$

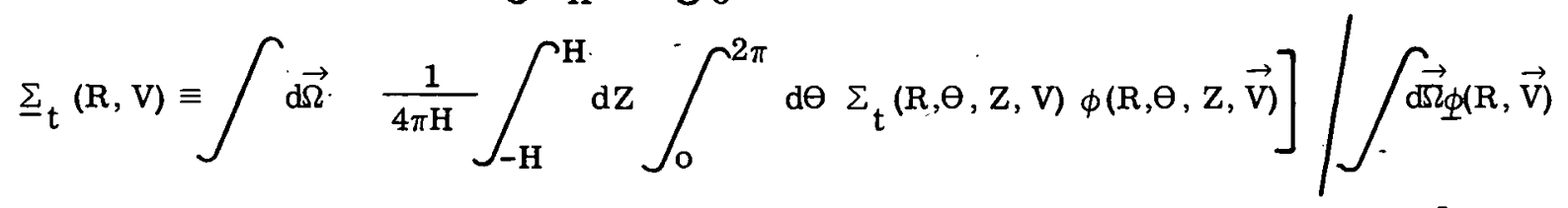

$$
\begin{aligned}
& \begin{array}{l}
L(R, \vec{V})=\frac{1}{4 \pi H} \quad \widehat{V} . \hat{z}\left[/ \phi(R, \theta, H, V) d \theta-\int \phi(R, \theta,-H, \vec{V}) d \theta\right] \text {, } \\
\text { et cetera. }
\end{array}
\end{aligned}
$$

There is a minor approximation implied in (5) in that the flux used as a weighting function must be averaged overall directions of motion of the neutron in the calculation of $\Sigma_{s}$, but this approximation should not introduce appreciable errors.

Equations (6) and (7) show how a three space dimensional cylindrical reactor may be studied in terms of a one-space dimensional neutron transport equation, if the behavior of the neutron flux as a function of the other spatial quantities is known from other 
considerations. The usual one dimensional diffusion approximation may be obtained from Equation (6). As a result of the averaging carried out in (3) and (4), this approximation is broadly valid because of the slowly varying nature of the functions involved. In RBU, the determination of the microscopic variations of the flux is made at infrequent intervals by the monte carlo method. From these microscopic fluxes are computed the averages of equation (7). These are then used in the principal neutron flux calculations made with the diffusion equation. 


\section{GENERAL OUTLINE OF THE RBU METHOD}

In RBU the calculation of the neutron flux is performed in two ways. Dependence on $\Theta$ and $\mathrm{Z}$ and the rapid variation of neutron flux within small ranges of energy and small volumes -- microscopic variations -- are computed by the monte carlo method. The gross dependence of flux on energy and radial distance from the cylindrical axis is computed using diffusion theory; the associated fluxes, regions, and groups are called macroscopic. The cross sections for macroscopic calculations are obtained in a manner similar to that outlined above by using the microscopic fluxes as weighting functions. Diffusion coefficients are obtained from the monte carlo results as the ratio of the net current leaving a macroscopic region to the normal gradient of the neutron flux at the surface bounding that region. Using this definition of the diffusion coefficient instead of the standard one involving the transport mean free path makes the integral of the diffusion equation over a region agree identically with the integral of the transport equation over the same region, and thus extends the validity of the diffusion approximation. The monte carlo calculations may be performed separately on isolated portions of the reactor, each containing a number of microscopic regions; such a portion is called a monte carlo system. The relationship between separate monte carlo systems is established by imposing as a boundary condition the requirement that the ratio between the emergent and incident neutron currents at the surfaces bounding the monte carlo systems agree with values tabulated for each macroscopic energy group. These ratios, called albedoes in this report (although they may be greater than unity), are determined from the macroscopic fluxes. The relationship between separate monte carlo systems is further established by using the macroscopic fluxes as normalizing functions for the fluxes in the microscopic regions represented.

The program may be instructed to modify the contents of a sequence of specified microscopic regionssequentially so that the reactor remains just critical as determined by a diffusion calculation. In this case the program iterates between the adjustment routine and the 
diffusion calculation until criticality is achieved. When a satisfactory diffusion calculation has been completed, the program prepares to determine the change in isotopic concentrations in some microscopic regions resulting from the exposure in the time steps which have taken place since the last burnup calculation on those regions. This involves the solution of a set of partial differential equations for isotope accretion and depletion. Not all microscopic regions are "burned up" after each time step. The number of time steps -that is, diffusion calculations -- between burnups is determined for each material by comparing the difference between two estimates of the concentrations with an upper and lower error tolerance limit. The program will thus adjust the time steps represented by burnup calculations for each material and, occasionally, will also adjust the time intervals represented by the diffusion calculations. Initial values of these time intervals are required as input and will be used until the program acquires sufficient information to change them.

When the isotopic concentrations in a region and, consequently, the microscopic fluxes have changed sufficiently to appreciably alter the effective average cross sections, it is desirable to repeat the monte carlo calculations to obtain new microscopic fluxes. The number of diffusion time steps between monte carlo calculations is specified by a number given as input for each monte carlo system.

The program continues cyclically-- consuming and accumulating isotopes, computing fluxes by diffusion theory to use in the isotope accretion and depletion calculation and re-evaluating the microscopic fluxes to use as weighting functions for the diffusion equations -- until an indication is reached that the calculation should be stopped. This indication is that either the maximum possible adjustments have been made in the compositions of all the microscopic regions in the sequence of control regions, or that the time variable has reached a predetermined value.

The general outline just described is shown as a flow chart in Figure 1. 


\section{THE MONTE CARLO PORTION OF RBU}

The RBU monte carlo is essentially similar to a previous monte carlo program, GMC. Modifications have been introduced in order to improve the speed and versatility of the program and to extend the treatment of such phenomena as resonances, neutron thermalization and anisotropic scattering.

\section{A. Geometry}

The geometric description of a monte carlo system is made by first specifying the parameters of the set of B surfaces bounding the regions in the system. A surface is identified by the index $b$. The surfaces which the program recognizes are of the form

$$
\mathrm{r} \equiv \mathrm{A}\left(\mathrm{x}-\mathrm{x}_{\mathrm{o}}\right)^{2}+\mathrm{B}\left(\mathrm{y}-\mathrm{y}_{\mathrm{o}}\right)^{2}+\mathrm{C}\left(\mathrm{z}-\mathrm{z}_{\mathrm{o}}\right)^{2}-\mathrm{K}=0
$$

In order to increase computing speed, certain special cases of (8) are specifically provided for: These are

$$
\begin{aligned}
& r \equiv\left(x-x_{0}\right)^{2}+\left(y-y_{0}\right)^{2}-K=0 \\
& r \equiv x-K=0 \\
& r \equiv y-K=0 \\
& r \equiv z-K=0
\end{aligned}
$$

Surfaces are specifically assigned one of the forms (8), (9), (10), (11), or (12) by associated with them a value of an index $D_{b}$ of $0,1,2,3$, or 4 respectively.

A microscopic region of space, $h$, is defined by listing the indices (values of $b$ ) for the surfaces bounding it. For each of these surfaces it is also necessary to specify whether an index $\mathrm{g}_{\mathrm{b}_{\mathrm{h}}}$ has the value plus or minus one. A surface $\mathrm{b}_{\mathrm{h}}$ of region $\mathrm{h}$ has $\mathrm{a}$ positive value of $g_{b_{h}}$ if the boundary function $r_{b}$ is negative for values of the coordinates $x$, $y$, and $z$ within region $h$ with respect to boundary $b$. The term $g_{b_{h}}$ is negative if $r$ is positive for values of $\mathrm{x}, \mathrm{y}$, and $\mathrm{x}$ inside region $\mathrm{h}$ with respect to $\mathrm{b}$. A simpler way to look at $\mathbf{g}_{h}$, and which works for most surfaces, is that it is positive for outer surfaces of a region and negative for inner surfaces. 
The boundary function is defined by one of the following equations depending on the value of $D$.

$$
r=\left\{\begin{array}{l}
A\left(x-x_{0}\right)^{2}+B\left(y-y_{0}\right)^{2}+C\left(z-z_{0}\right)^{2}-K \\
\left(x-x_{0}\right)^{2}+\left(y-y_{0}\right)^{2}-K \\
y-K \\
x-K \\
o r \\
z-K
\end{array}\right.
$$

Additional quantities associated with a region are: an index $\mathrm{m}_{\mathrm{h}}$, specifying the material contained in region $h$; a value of $I_{h}$, the importance of region $h, k_{h}$, the macroscopic region within which $h$ is contained; $B_{h}$, the total number of bounding surfaces used to described $\mathrm{h} ; \perp_{\mathrm{b}_{\mathrm{g}}}$, which indicates whether or not leakage from region $\mathrm{h}$ through boundary $b$ contributes to the perpendicular leakage to be used for region $k_{h}$ in the diffusion calculation and $\mathrm{Q}_{\mathrm{b}_{\mathrm{h}}}, \mathrm{Q}_{\mathrm{b}_{\mathrm{h}}^{\prime}}$, indices of the microscopic regions (in order of likelihood) which a neutron leaving region $h$ through boundary $b$ will probably enter.

Region $h$ belongs to a set, $f_{h}$, of microscopic regions. The neutron fluxes computed in the monte carlo are listed as the fluxes, $\phi_{\text {if }}$ in group $i$, region set $\mathrm{f}$.

B. Description of a Material

As mentioned above, the description of a region, $h$, includes an index, $m_{h}$, which determines which of the $M$ mixtures of isotopes is contained in region $h$. The information about a material required by the monte carlo portion of RBU is collected and computed from a basic detailed library of isotopic properties, and from the input by two portions of the program, the Cross Section Averaging Routine and the Pre-Monte Carlo. These portions of the program, described in later sections of this report, assemble the nuclear data for each material and each microscopic energy group, i. In brief these data consist of mean free paths, $\lambda_{\mathrm{mi}}$; emission probabilities for the pertinent collision types, $P_{c_{\mathrm{mi}}}$; atomic 
weights of the scatterers, $a_{\mathrm{mi}}$; prescriptions for the inelastic scattering spectra; probability distributions for velocity increments for near thermal scattering; parameters for the resonances for those scatterers in the material which have them; and finally coefficients for anisotropic scattering.

\section{General Outline of the Monte Carlo Portion}

Particles -- that is, the position, velocity, and time coordinates of particles -are followed for an interval of time from 0 to $\mathrm{T}$ or until they are terminated before $\mathrm{T}$ by capture or by escape from the system. The determination of variables, such as distance between collisions and directions and velocities after collision is made by random selection from the relevant probability distributions. At each collision, analytic calculations are made of the values of certain averages for a universe of neutrons with the coordinates with which particles emerge from the collision. These averages are the expectation values of the first flight contributions for the monte carle time interval of such quantities of interest as the neutron flux in each microscopic group, $i$, and region set, $f_{z}$ and the currents and normal gradients of the currents at the boundaries of macroscopic regions in each macroscopic group. Thus, the monte carlo method is used to establish a collision distribution from which the quantities of interest are computed analytically.

To each microscopic group and region, an importance is assigned by the input; the number of neutrons represented by a particle in region $h$ and group $i$ is $\frac{1}{I_{j} I_{h}}$, the reciprocal of the product of the importances of the group and region in which it is. Since neutrons are conserved when particles cross from one group or region to another, the effect of this is to make the intensity with which a group and region is studied a function of its importance as well as of the actual neutron population.

The particles to be studied are obtained initially from a tape containing those which reached census time, $T$, in the previous monte carlo time step for this system. For the first time step, of course, they must be obtained elsewhere; they may either be particles saved from previous calculations or particles generated by a special generator routine. 
A period of time between 0 and $\mathrm{T}_{0}$, during which the particles are followed without recording their history, is inserted in order to permit the population to come into equilibrium with the configuration of the system. In order to accelerate this process, the number of particles in each region and group actually used is modified from the census of the previous time step according to the variation in flux between previous monte carlo calculations of this system.

D. Sampling From. Initial Neutron Tape

On reading a particle from the initial neutron (I.V.) tape, the program first checks to be sure that the region $\mathrm{h}$ indicated by its coordinates is actually the region in which the particle is located.

The condition that a particle be in zone $h$ is that, for all boundaries, $b_{h}$ of $h$

$$
\mathrm{g}_{\mathrm{b}} \cdot \mathrm{r}_{\mathrm{b}}(\mathrm{x}, \mathrm{y}, \mathrm{z}) \leq 0
$$

The method used to determine whether or not a set of coordinates lies within a region imposes a limitation on the possible region shapes. Generally speaking, this is that no two boundaries of a region may meet in such a way as to produce a concavity. This does not affect the generality of the geometry since a set of regions may have any shape. If the particle is not in zone $\mathrm{h}$, the program tests all other regions to determine which region it is in.

The procedure of checking to be sure that a particle read from the I. V. tape is actually in the indicated zone is inserted in order to make it possible to interchange I. V. tapes between problems. During the normal operation of $\mathrm{RBU}$ and in particular after the first monte carlo time step, the zone index associated with a particle may be assumed to be correct. The program, in fact, will insist upon this and will bypass the zone checking routine unless the monte carlo system number is negative.

Having determined the correct region number and substituted this for $h$, the program next decides how many times this set of initial coordinates is to be followed. Included in a set of coordinates is a number, $N$, the number of particles having the exact values of 
$\mathbf{x}, \mathbf{y}, \mathbf{z}, \mathrm{V}, \alpha, \beta$, and $\gamma$, given in that set. The term $\mathbf{N}$, in other words, is the number of particles represented by a set of coordinates. The fact that $\mathrm{N}$ may be different from unity is a result of the sampling procedure about to be described and of the manner in which particles come to be written on the census tape. The modification of $\mathrm{N}$, on reading a set of coordinates from the I. V. tape is made to accomplish two purposes.. First, the distribution of particles on the I. V. tape is to be distorted in order to accelerate the approach to equilibrium. This introduces a factor $A_{\text {if }}^{t}$ in the number of particles to be made of the particle read from the I. V. tape. (The superscript $t$ indicates the monte carlo time step number.) Secondly, the total number of particles to be followed is to be adjusted to approximate the number, $\mathrm{N}_{\mathrm{MC}}$ which the programmer considers to be necessary for the required statistics. $\mathrm{N}$, then, is changed to

$$
=_{\mathrm{if}}^{\mathrm{t}} \frac{\mathrm{N}_{\mathrm{MC}}}{\mathrm{N}_{\mathrm{I} . \mathrm{V} .}^{\mathrm{t}}} \mathrm{N}+\xi=
$$

The symbol $€ \neq$ signifies "greatest integer in" and $\xi$ is a random number (see

Section XIII A).By adding a random number and taking the greatest integer, the program selects integral values of quantities in such a way that the average of many such selections approaches the appropriate number -- in this case

$$
A_{\text {if }}^{t} \frac{N_{M C}}{N_{\text {I. V. }}^{t}} N
$$

The term $\mathrm{N}_{\mathrm{I}}^{\mathrm{t}} \mathrm{V}$. is the total number of particles on the I. V. tape. It may be assumed to be equal to the number of particles which reached census time in the previous monte carlo time step. Actually the multiplication by $A_{\text {if }}^{t} \frac{\mathrm{N}_{M C}}{N_{\text {I. V. }}^{t}}$ is done at the end of the previous. time step. The only part of equation (30) performed at the time a particle is read from the I. V. tape is the addition of a randum number and the selection of the largest integral in the sum. 


\section{E. Unresolved Resonances}

The next stage of the monte carlo program involves the knowledge of the mean free paths in the region in which the particle has just been found to be. In most cases this is obtained from tables prepared in the pre-monte carlo routine. Some materials, however, contain isotopes the cross sections of which are, for part of the energy range of interest, most conveniently described by parameters specifying single level Breit Wigner resonances -or distributions of those parameters. For each of these resonance isotopes, there are two energy ranges in which the Breit Wigner description is used. In the lower: (resolved) range, a table of values of resonance parameters, for particular resonances in that isotope, specify the cross section. In the higher (unresolved) range, the resonance parameters are determined by selection from appropriate probability distributions. In the unresolved range, the quantities specified are:

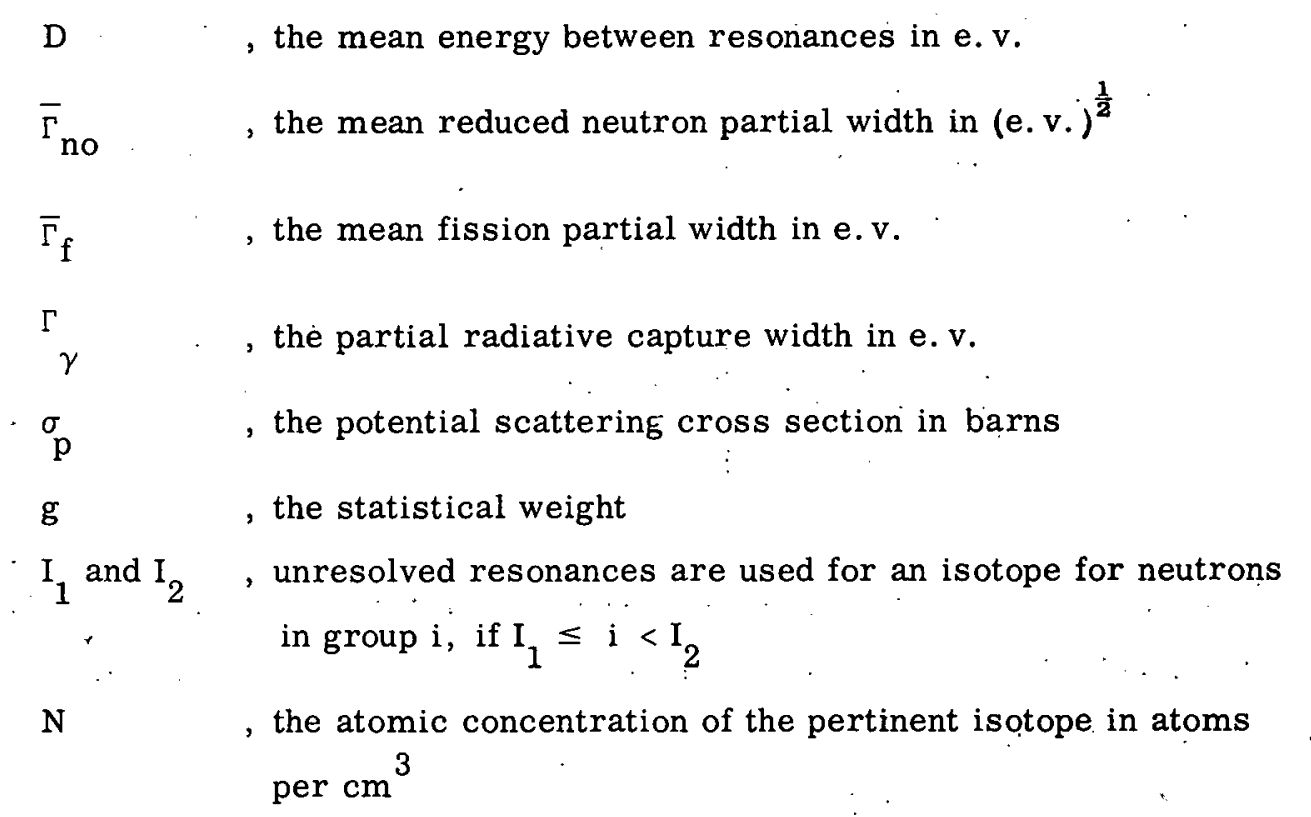

The values of these are specified for each of the resonance isotopes in the region. For each of these isotopes the actual value of the cross sections seen by a particle at an individual event is determined by random selection from the appropriate probability distributions. The probability that 


$$
u \equiv\left(\frac{\Gamma_{\text {no }}}{\bar{\Gamma}_{\text {no }}}\right)^{\frac{1}{2}}
$$

be between $u$ and $u+d u$ is given by the Porter-Thomas distribution

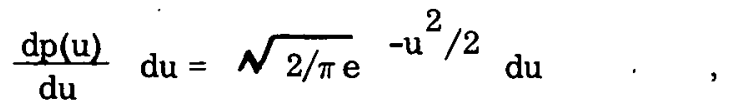

and the probability that

$$
\mathrm{v} \equiv \Gamma_{\mathbf{f}} / \bar{\Gamma}_{\mathbf{f}}
$$

be between $v$ and $v+d v$ is

$$
\frac{d P(v)}{d v} d v=e^{-v} d v
$$

The manner.in which values of $\Gamma_{\mathrm{n}_{\mathrm{o}}}$ and $\Gamma_{\mathrm{f}}$ are selected from these distributions is described in Section XIII $\mathrm{A}_{\gamma} \mathrm{I}_{\gamma}$ is assumed to be constant for all unresolved resonances for a particular isotope.

The cross section at $\mathrm{E}$, the energy of the neutron, is approximated by the sum of those contributed independently by two resonances whose peaks lie at

$$
\mathrm{E}_{\mathrm{o}_{\mathrm{a}}}=\left[\frac{\frac{a}{a+1} \mathrm{v}^{2}}{1.9132 \mathrm{D}} \neq \mathrm{D}\right. \text { and }
$$

where $\mathrm{V}$ is the neutron velocity in $\mathrm{cm} /$ microsecond. Values of the partial widths are selected for each of the two resonances as described above and the cross section due to each is evaluated from

$$
\begin{array}{cc}
\Gamma_{\mathrm{n}}=\Gamma_{\mathrm{n}}, \sqrt{\mathrm{E}} & \text { in e.v. } \\
\Gamma=\Gamma_{\gamma}+\Gamma_{\mathrm{f}}+\Gamma_{\mathrm{n}}, & \text { in e.v. } \\
\theta=1.7987 \times 10^{-4} \frac{\mathrm{v}^{2} \mathrm{~T}}{Q \Gamma^{2}}\left(\frac{Q}{Q+1}\right){ }_{-16}^{2} & \text { (dimensionless) }
\end{array}
$$




$$
\mathrm{X}=2\left[\frac{\mathrm{v}^{2}}{1.9132} \frac{a}{a+1}-\mathrm{E}_{0}\right] / \Gamma \quad \text { (dimensionless) }
$$

where $Q$ is the mass number of the resonance isotope and $T$ is the temperature of the material in degrees Kelvin; from

$$
\begin{gathered}
\sigma_{\mathrm{s}_{\mathrm{o}}}=2.6035 \times 10^{6} \mathrm{~g}\left(\frac{1+a}{a}\right) \frac{\Gamma_{\mathrm{n}}^{2}}{\mathrm{E}_{\mathrm{o}} \Gamma^{2}}\left(1+\mathrm{D}^{*} \mathrm{v}^{2}\right)\left(1+l^{*} \mathrm{~V}\right)^{2} \\
\sigma_{\mathrm{c}}=3.6006 \times 10^{6} \mathrm{~g}\left(\frac{1+a}{a}\right)^{3 / 2} \frac{1}{\mathrm{~V} \sqrt{\mathrm{E}}} \frac{\Gamma_{\mathrm{n}} \Gamma_{\gamma}}{\Gamma^{2}}\left(1+\mathrm{D}^{*} \mathrm{v}^{2}\right)\left(1+\ell^{*} \mathrm{~V}\right)^{2} \\
\sigma_{\text {fo }}=\sigma_{\mathrm{co}} \frac{\Gamma_{\mathrm{f}}}{\Gamma_{\mathrm{g}}}
\end{gathered}
$$

and from

$$
\begin{aligned}
& \sigma_{\mathrm{s}}=\sigma_{\mathrm{s}_{\mathrm{o}}} \psi(\mathrm{X}, \theta)+2 \sqrt{\sigma_{\mathrm{p}} \mathrm{g} \sigma_{\mathrm{s}_{\mathrm{o}}}} \quad \chi(\mathrm{X}, \theta)+\sigma_{\mathrm{p}} \\
& \sigma_{\mathrm{c}}=\sigma_{\mathrm{c}} \psi(\mathrm{X}, \theta) \\
& \sigma_{\mathrm{f}}=\sigma_{\mathrm{f}} \psi(\mathrm{X}, \Theta) \\
& \text { (barns) }
\end{aligned}
$$

The factors $\frac{\mathrm{D}^{\prime}}{\mathrm{D}}=1+\mathrm{D}^{*} \mathrm{~V}^{2}$ and $\ell^{\prime} / \ell=\left(1+\ell^{*} \mathrm{~V}\right)^{2}$ are rough approximations included to account for the variation of level density with energy and for the presence of resonances with angular momentum higher than $\ell=0 . \mathrm{D}^{*}$ and $\ell^{*}$ are constants specified for each each isotope in the Basic Library, the broadened resonance functions are defined by

$$
\psi(\mathrm{X}, \theta)=\frac{1}{2 \sqrt{\pi \theta}} \int_{-\infty}^{\infty} \frac{\mathrm{e}^{-\left[\frac{(\mathrm{X}-\mathrm{Y})^{2}}{4 \theta}\right]}}{1+\mathrm{Y}^{2}} \mathrm{dY}
$$




$$
\chi(X, \theta)=\frac{1}{2 \sqrt{\pi \theta}} . \quad \frac{Y e^{-\frac{(X-Y)^{2}}{4 \theta}}}{1+Y^{2}} d Y
$$

The evaluation of $\psi$ and $\chi$ is described in Section XIII A and the derivation of (22) and (23). is described in Section XIIIC.

\section{F. Resolved Resonances}

Resolved resonances are treated in a manner similar to that described above for unresolved resonances. The difference is that the values of the parameters, $E_{0}, \Gamma{ }_{n}$, and $\Gamma_{f}$ are specified. For neutrons in velocity groups between $I_{3 w m}$ and $I_{2 w m}$, the cross sections for the $w^{\text {th }}$ resonance scatterer in material $\mathrm{m}$ are computed using Equation (22) with values of $\mathrm{E}_{\mathrm{o}}, \Gamma_{\mathrm{n}}, \Gamma_{\gamma}, \mathrm{g}$, and $\Gamma_{\mathrm{f}}$ obtained from one of the lists of resonance parameters stored in the memory. An index e associated with the $\mathrm{w}^{\text {th }}$ resonance scatterer of material $m$ identifies the list to be used for it. As in the unresolved region, the cross sections computed are the sum of independent contributions of two resonances, these being the ones with values of $E_{0}$ on either side of the reduced energy, $\frac{v^{2} \frac{a}{a+1}}{1.9132}$ of the neutron. The values of the other resonance parameters are the same as those used in the unresolved region for the same scatterer. For neutrons within the resolved range but above the highest resolved resonances, the lower energy resonance used in the highest one is the target isotopes resolved table, while the higher energy resonance is chosen as in the unresolved range. For neutrons of energy below the lowest resolved resonance but within the resolved range, only one resonance, the lowest one in the table, is used.

\section{G. Between Collisions Routine}

In this stage of the calculation, a group of $\mathrm{N}$ particles, each having identical coordinatés, which have resulted either from a collision or from selection from the $\mathrm{I}$. V. tape, are followed to their individual next collisions, to census time, or to loss, through leakage, from the system. In the course of this process, the expectation values of the 
contributions of these particles to quantities of interest are computed region by region for the enetire path the particles have the possibility of following in first flight until they leak or reach census time. The distance a particle at position $x, y, z$ traveling in direction $\alpha, \beta, \gamma$ will have to go to reach boundary $\mathrm{b}$ of region $\mathrm{h}$ is

where

$$
s_{b}=\frac{-e+g_{b h} \sqrt{e^{2}-r}}{h}
$$

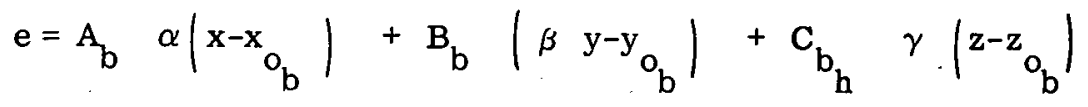

$$
\begin{aligned}
& =\mathrm{A}_{\mathrm{b}} \quad \alpha^{2}+\mathrm{B}_{\mathrm{b}} \cdot \beta^{2}+\mathrm{C}_{\mathrm{b}} \gamma^{2} \\
& r=A_{b}\left(x-x_{o_{b}}\right)^{2}+B_{b}\left(y-y_{o_{b}}\right)^{2}+c_{b}\left|z-z_{o_{b}}\right|^{2}-K_{b}
\end{aligned}
$$

For the special boundaries of Equations (9) through (12) these formulas reduce to

$$
\begin{aligned}
& \mathrm{e}=\alpha\left|\mathrm{x}-\mathrm{x}_{\mathrm{o}_{\mathrm{b} .}}\right|+\beta\left(\mathrm{y}-\mathrm{y}_{\mathrm{o}_{\mathrm{b} .}}\right) \\
& =\alpha^{2}+\beta^{2} \\
& r=\left|x-x_{o_{b}}\right|^{2}+\left(y-\left.y_{o_{b}}\right|^{2}-k_{b}\right. \\
& \mathrm{S}_{\mathrm{b}}=-\frac{\mathrm{x}-\mathrm{K}}{\alpha} \text { if } \frac{\mathrm{x}-\mathrm{K}}{\alpha}<0 \\
& \mathrm{~S}_{\mathrm{b}}=-\frac{\mathrm{y}-\mathrm{K}}{\beta} \text { if } \frac{\mathrm{y}-\mathrm{K}}{\beta}<0 \\
& \mathrm{~S}_{\mathrm{b}}=-\frac{\mathrm{z}-\mathrm{K}}{\gamma} \text { if } \frac{\mathrm{z}-\mathrm{K}}{\gamma}<0 \\
& D=1
\end{aligned}
$$


The distance to boundary is computed by means of one of Equations (24) through (27) for each of the boundaries of the region in which the particle is, the smallest positive value so obtained being accepted as the actual distance. In order to minimize computing time on calculations involving (24) (that is when $\mathrm{D}_{b}=0$ or 1), Sturm's Theorem of the locations of roots of a quadratic equation is used to decrease the number of boundaries for which the calculation is actually made.

The between collisions routine begins with a set of $\mathrm{N}$ particles at a particular position and with a particular velocity. These represent $\frac{N}{I_{j h}}$ neutrons where $I_{j}$, and $I_{h}$ are the importance of group $\mathrm{j}$ and the importance of region $\mathrm{h}$ respectively. By random selection from the appropriate distribution, a distance to collision is determined for each of the $\mathrm{N}$ particles. These are the distances each will go before having a collision provided it does not first cross a boundary into a new region or reach census time. For each of those particles which have a collision before leaving the region, the program transfers to the collision routine, computes the new velocity and direction, and stores the coordinates of the resultant particles in a region of memory known as born storage. At an appropriate stage of the program, they will be followed further. The remaining particles (those which have not had collisions in the region in question) are advanced just over the boundary into the next region, provided they do not first reach census time, and $\mathbf{N}$ is diminished by the number which had failed to reach the boundary. In order to insure that the particles do get past the boundary when they are advanced, the distance to boundary is multiplied by $\delta$, a number slightly greater than unity, when used in the equation for the new coordinates of the particle. Associated with this set of particles is a number, $\pi$, representing the expectation value of the number of neutrons of the set reaching the region in which they are being considered. This is initially set to $\frac{N}{I_{h} I_{j}}$ when the set of particles is obtained from born storage or the I. V. tape, and is diminished by the factor $e^{-S} b^{/ \lambda}$ each time the program advances the particles a distance $S_{b}$ through a region. 
The expectation value of the path length (which is, of course, proportional to the flux) swept out by particles starting at a distance $S$ from the boundary of a region is

$$
\phi_{\text {if }}=\lambda \operatorname{Tr}\left(1-\mathrm{e}^{-\mathrm{S} / \lambda}\right)
$$

Since the quanity of interest here, however, is the track length during the period $\mathrm{T}_{\mathrm{o}} \leq \mathrm{t} \leq \mathrm{T},(28)$ is modified by using for $\mathrm{S}$ the distance the particle could possibly travel in that period in region $\mathrm{h}$ and by multiplying by the attenuation taking place during the period (if any) during which $\mathrm{t}<\mathrm{T}_{\mathrm{o}}$. Thus

$$
\phi_{\text {if }}=\lambda \pi \mathrm{p}_{1}\left(1-\mathrm{p}_{2}\right)
$$

where

$$
\begin{aligned}
& p_{1}= \begin{cases}\exp -\frac{\left(T_{0}-t\right) V}{\lambda} & \text { if } t<T_{0} \text { and }\left(T_{0}-t\right) V<S_{b} \\
0 & \text { if } t<T_{0} \text { and }\left(T_{0}-t\right) V \geq S_{b} \\
1 & \text { if } t \geq T_{0}\end{cases} \\
& p_{2}= \begin{cases}e^{-S_{b} / \lambda} & \text { if }(T-t) V \geq S_{b} \text { and } t \geq T_{0} \\
e^{-(T-t) V / \lambda} & \text { if }(T-t) V<S_{b} \text { and } t \geq T_{0} \\
e^{-\left(T-T_{0}\right) \cdot V / \lambda} & \text { if }(T-t) V<S_{b} \text { and } t<T_{0}\end{cases} \\
& e^{-\left(S_{b}-\left(T_{0}-t\right) V / \lambda \mid\right.} \text { if }(T-t) V \geq S_{b} \text { and } t<T_{0}
\end{aligned}
$$

The average cross sections to be used in the diffusion and burnup calculations for the isotopes having resonances are obtained from expectation value calculations also. For a particle in macroscopic group $\mathrm{j}$ in a region containing material $\mathrm{m}$, the appropriate tallies 


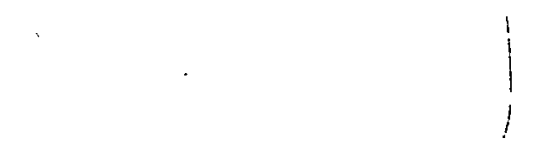

for the $w^{\text {th }}$ resonances isotopes of material $m$ are

$$
\begin{aligned}
& \left(\Sigma_{\mathrm{s}} \phi\right)_{\mathrm{w}_{\mathrm{m}} \mathrm{j}}=\pi \mathrm{p}_{1}\left(1-\mathrm{p}_{2}\right) \Sigma_{\mathrm{s}} \lambda \\
& \left(\Sigma_{\mathrm{f}} \phi\right)_{\mathrm{w}_{\mathrm{m}} \mathrm{j}}=\pi \mathrm{p}_{1}\left(1-\mathrm{p}_{2}\right) \Sigma_{\mathrm{f}^{\prime}} \\
& \left(\Sigma_{\mathrm{c}} \phi\right)_{\mathrm{w}_{\mathrm{m}} \mathrm{j}}=\pi \mathrm{p}_{1}\left(1-\mathrm{p}_{2}\right) \Sigma_{\mathrm{c}} \lambda
\end{aligned}
$$

For particles crossing from one macroscopic region to another, the expectation value of the components of the neutron current and of the gradient of the neutron flux normal to the interface between these regions are also tallied. These tallies are useditocompute the diffusion coefficients. The appropriate quantities for particles reaching the interface between macroscopic regions $k$ and $k^{\prime}$ in macroscopic group $\mathrm{j}$ are

$$
\begin{aligned}
& \mathrm{L}_{k j}=\pi\left(1-\omega_{b j}\right)=-L_{k^{\prime} j} \\
& (\hat{n} \cdot \vec{\nabla} \phi)_{k j,}, \quad \pi\left(1-c_{b j}\right), \frac{\Delta}{\lambda}=-(\hat{n} . \vec{\nabla} \phi)_{k^{\prime} j} \\
& \Delta=x+\delta+\rho
\end{aligned}
$$

Where $\lambda$ is the mean free path for the microscopic group and 'region in which the particle is when it reaches the boundary, $\omega_{b j}$ is the albedo for boundary $b$ and macroscopic group j.

$$
\begin{aligned}
x & =\mathrm{A} \quad \alpha\left(\mathrm{s}-\mathrm{s}_{\mathrm{o}}\right) / \mathscr{D} \\
\delta & =\mathrm{B} \beta\left(\mathrm{y}-\mathrm{y}_{\mathrm{o}}\right) / \mathscr{D} \\
\rho & =\mathrm{C} \gamma\left(\mathrm{z}-\mathrm{z}_{\mathrm{o}}\right) / \mathscr{D} \\
\mathscr{D} & =\left[\alpha^{2} \mathrm{~A}^{2}\left(\mathrm{x}-\mathrm{x}_{\mathrm{o}}\right)^{2}+\beta^{2} \mathrm{~B}^{2}\left(\mathrm{y}-\mathrm{y}_{\mathrm{o}}\right)^{2}+\gamma^{2} \mathrm{C}^{2}\left(\mathrm{z}-\mathrm{z}_{\mathrm{o}}\right)^{2}\right]^{\frac{1}{2}}
\end{aligned}
$$


$A, B, C, x_{o}, y_{o}, z_{o}$ are the parameters for the relevant boundary. For boundaries of the special forms, $\mathrm{D}=2,3$, or 4 ,

$$
\begin{aligned}
& D=1 \quad \chi=\alpha\left(x-x_{0}\right) / \mathscr{D} \\
& \delta=\beta\left(y-y_{0}\right) / \varnothing \\
& \rho=0 \\
& \mathscr{D}=\left[\left(\mathrm{x}-\mathrm{x}_{0}\right)^{2}+\left(\mathrm{y}-\mathrm{y}_{\mathrm{o}}\right)^{2}\right]^{\frac{1}{2}} \\
& \mathrm{D}=2 . \quad x=\alpha \\
& \delta=\rho=0 \\
& \mathrm{D}=3 \quad \delta=\beta \\
& x=\rho=0 \\
& \mathrm{D}=4 \quad \chi=\rho=0 \\
& \rho=\gamma
\end{aligned}
$$

The expectation value of the leakage out of the system from microscopic region $h$ through boundary $b_{h}$ is tallied separately for those region-boundary combinations for which the quantity $\perp_{b_{h}}$ in the input is non-zero. These tallies, used to obtain the perpendicular leakage term in Equation (6), are recorded by macroscopic region and group

$$
\mathcal{L}_{\mathrm{kj}}=\pi\left(1-\omega_{\mathrm{bj}}\right)
$$

When the monte carlo program is used as a part of RBU, it will generally be true that $\omega_{b j}$ is zero for any boundary for which $\perp_{b_{h}}$ is not zero and vice versa.

Certain other quantities are also computed, some of these primarily for code and input checking purposes.

$\mathrm{P}_{\text {I. V. }}: \quad$ Number of sets of initial coordinates followed

$\mathrm{N}_{\text {I. V. }} \quad$ Number of initial neutrons followed 
$n_{\text {I. V. }}$ Number of initial particles followed

Reject

Particles : Number of particles rejected as initially outside the system

Reject

Neutrons: Number of neutrons rejected as initially outside the system

$P_{c v}: \quad$ Number of sets of coordinates reaching census time

NC.V. Number of neutrons reaching census

$\eta_{\text {c. v. }}$ Number of particles reaching census

$\mathrm{N}_{\mathrm{To}_{0}}: \quad$ Number of neutrons reaching $\mathrm{T}_{\mathrm{o}}$.

$n_{\text {To: }} \quad$ Number of particles reaching $T_{0}$

$\left\langle\mathrm{N}_{\mathrm{To}_{0}}\right\rangle: \quad$ Expectation value of number of neutrons reaching To

$<\mathrm{N}_{\mathrm{BO}}>$ : Expectation value of the number of neutrons escaping from the system by leakage

$\mathrm{N}_{\mathrm{BO}}$ : Number of neutrons escaping from the system by leakage

$\mathrm{N}_{\mathrm{B}}$ : $\quad$ Net number of neutrons lost at internal boundaries

(due to statistical fluctuations associated with the use of weights)

$\mathrm{N}_{\mathrm{BW}}$ : Number of neutrons lost at albedo surfaces

$\eta_{\mathrm{c}}: \quad$ Number of particles entering collision

$\mathrm{N}_{\mathrm{C}}: \quad$ Number of neutrons entering collision

$<\mathrm{N}_{\mathrm{c}}$ : $\quad$ Expectation value of the number of neutrons entering collisions

$<\mathrm{Ne}>$ : $\quad$ Expectation value of the number of neutrons emerging from collisions

$\left\langle\mathrm{Ne}^{\prime}\right\rangle$ : $\quad$ Sum overall collisions taking place of the mean number of emergent neutrons for that collision.

$\mathrm{Ne}$ : Number of neutrons emerging from collision 
There are the quantities referred to as "code check quantities",

As described above, for each particle a determination is made individually of whether or not the particle is to have a collision before reaching the boundary of the region it is crossing The determination is made by random selection of $\mathrm{S}_{\mathrm{c}}$ from the distribution.

$$
\frac{\mathrm{dP}\left(\mathrm{S}_{\mathrm{c}}\right)}{\mathrm{dS} \mathrm{c}_{\mathrm{c}}}=-\frac{1}{\lambda} \mathrm{e}^{-\mathrm{S}_{\mathrm{c}} / \lambda} .
$$

For each particle for which $S_{c}$ is less than both $S_{b}$ and $S_{T}=(T-t) V$, the program transfers to the collision routine in which the results of the collision are determined. The coordinates of any particles emitted in the collision are stored temporarily in a region of memory known as born storage and the program returns to the between collisions routine to process the remaining particles and the remainder of the undeflected path.

\section{H. Collision Routine}

Among the material data, as was mentioned previously, is a set of numbe ${ }^{\mathbf{P}_{\mathrm{c}}} \mathrm{mi}_{\mathrm{mi}}$, the number of neutrons produced due to collision process $\mathrm{c}$ per neutron colliding in material $\mathrm{m}$ and microscopic group $\mathrm{i}$. The different values of the index correspond to the different collision types, elastic, inelastic and fission and (where necessary as in the use of elastic collisions with light nuclei and inelastic collisions where the scatterers present have different inelastic scattering spectra) to the different isotopes present.

On entering the collision routine the program determine whether or not to select new coordinates for particles to be emitted by a collision process. This decision depends on whether or not

$$
\mathrm{N}^{\prime}=\left\{\mathrm{P}_{\mathrm{c}_{\mathrm{mi}}}+\xi \neq .\right.
$$

is zero.

The program then proceeds, if $N^{\prime} \neq 0$, to determine the direction and velocity of the emergent particles by methods dependent on the collision type and to be described below. 
In the event that a change in velocity takes place, it may be necessary to modify $\mathrm{N}^{\prime}$ when the new velocity is known because of the group weights. If $I_{j}$ and $I_{j}$, are respectively the importance of the group in which the particle is incident and that of the one in which it emerges

$$
N=f \begin{aligned}
& I_{j \prime} \\
& I_{j}
\end{aligned} N^{\prime}+\xi \neq
$$

If $\mathrm{N}$ is not zero the particle's coordinates are stored in born storage and the program considers the collision corresponding to the next value of $\mathrm{c}$.

Radiative capture collisions are automatically accounted for in this process by their effect in diminishing the value of $\sum_{c} P_{c}$ mi . The other collision types will now be discussed.

\section{Elastic Scattering}

The simplest case for elastic collisions occurs for heavy scatterers when the energy of the incident neutron is below the minimum (specified by I") for anisotropic scattering and when it is not-in a range in which moderation by heavy nuclei is considered. Under these circumstances, that is when

$$
\begin{aligned}
& a_{r}>a_{h} . \\
& I_{\mathbf{m}}>\text { i or } \mathrm{i}>\mathrm{I}_{L_{\mathrm{m}}} \\
& \mathrm{i}>\mathrm{I}_{\text {aniso }}
\end{aligned}
$$

the neutron's velocity does not change. The emergent direction cosines are selected at random from an isotropic universe as described in section XIII A. In (38), $a_{r}$ is the atomic weight of the scatterer; $a_{h}$ is the atomic weight defining light and heavy scatterers; $I_{\text {aniso }}$ is the lowest energy group (highest group number) in which anisotropic scattering is considered for $\mathrm{r}^{\text {th }}$ scatterer and $\mathrm{I}_{\mathrm{H}_{\mathrm{m}}}$ and $\mathrm{I}_{\mathrm{L}_{\mathrm{m}}}$ are the upper and lower energy group limits (lower and upper group numbers) of the range in which heavy scatterers are per mitted to moderate the neutrons. 
If either of the first two conditions of (38) fail, the relationship between the center of mass and laboratory systems must be taken into account. In the isotropic case, random direction cosines $\alpha^{\prime \prime}, \beta^{\prime \prime}$, and $\gamma^{\prime \prime}$ are chosen from an isotropic distribution and the value of $\mu$, the cosine of the angle between the incident and emergent directions in the center of mass system is determined.

$$
\mu=\alpha \alpha^{\prime \prime}+\beta \beta^{\prime \prime}+\gamma \gamma^{\prime \prime}
$$

The emergent direction cosines in the laboratory system, $\alpha^{\prime}, \beta^{\prime}, \gamma^{\prime}$, and the emergent velocity $V^{\prime}$ are then calculated from

$$
\begin{aligned}
& \alpha^{\prime}=\left(a_{\mathrm{r}} \alpha^{\prime \prime}+\alpha\right) / \mathrm{c} \\
& \beta^{\prime}=\left(a_{\mathrm{r}} \beta^{\prime \prime}+\beta\right) / \mathrm{C} \\
& \gamma^{\prime}=\left(a_{\mathrm{r}} \gamma^{\prime \prime}+\gamma\right) / \mathrm{C} \\
& \mathrm{V}^{\prime}=\mathrm{vC} /\left(1+a_{\mathrm{r}}\right)
\end{aligned}
$$

where

$$
\mathrm{c}=\left[1+a_{\mathrm{r}}^{2}+2 \mu a_{\mathrm{r}}\right]^{\frac{1}{2}}
$$

In order to conserve computing time and storage space for the $\mathrm{P}_{\mathrm{c}_{\mathrm{mi}}}$, the same value of $a_{\mathrm{r}}$ is used for all heavy scatterers in (40). This permits the lumping of all heavy scatterers in one value of $\mathrm{c}$. The atomic weight normally used for these is 238 .

When scattering is anisotropic, that is when $\mathrm{i}<\mathrm{I}_{\text {aniso }}$, a simple modification causes the selection of the angle of scattering in the center of mass system to be made from

$$
\frac{\mathrm{dP}(\mu)}{\mathrm{d} \mu}=\mathrm{a}_{0}+\mathrm{a}_{1} \mu+\mathrm{a}_{2} \mu^{2}+\mathrm{a}_{3} \mu^{3} .
$$

The method is to compute $\mu$ from

$$
\mu=\alpha \alpha^{\prime \prime}+\beta \beta^{\prime \prime}+\gamma \gamma^{\prime \prime}
$$

as above, but to accept the random cosines $\alpha^{\prime \prime}, \beta^{\prime \prime}, \gamma^{\prime \prime}$ only if

$$
\xi a_{m} \leq a_{0}+a_{1} \mu+a_{2} \mu^{2}+a_{3} \mu^{3}
$$


In (43), $\xi$ is a number chosen at random from a uniform distribution between zero and one. If (43) is not satisfied by $\mu$ and $\xi$, a new set $\alpha^{\prime \prime}, \beta^{\prime \prime}, \gamma^{\prime \prime}, \xi$ are chosen. Thus, the probability of accepting a direction is proportional to (41). The values of $a_{0}, a_{1}, a_{2}$, $a_{3}$, and $a_{m}$ used in (43) are found in the listings of material data separated according to microscopic energy group and scatterer. The term $a_{m}$ is the largest possible value of $\mathrm{dP}(\mu) / \mathrm{d} \mu$ for $-1 \leq \mu \leq 1$ and is computed in the portion of the program in which the monte carlo constants are calculated. For the collective heavy scatterer in a material only one set of $a_{\ell}$ is stored for each group, this being a concentration and cross section weighted average over the heavy scatterers present computed by the pre-monte carlo portion of the program.

If moderation is unimportant $\alpha^{\prime \prime}, \beta^{\prime \prime}, \gamma^{\prime \prime}$ are used as the new laboratory system direction cosines; if it is to be considered, as determined by the first two of Equation (38), $\alpha^{\prime \prime}, \beta^{\prime \prime}$, and $\gamma^{\prime \prime}$ are used to compute the laboratory system direction cosines and velocity by (40).

When the energy of the particle is comparable with the thermal kinetic energy of the scattering nuclei, two further modifications are made to order to account for the effect of molecular and crystal binding of the scattering atoms and the effect of their thermal motion. The first of these is that, if the incident velocity is such that $i>I-G_{v}$, the atomic weight used in (40) is permitted to vary with the incident microscopic group. Secondly, if the emergent velocity in the center of mass system is less than $\mathrm{V}_{\mathrm{T}_{\mathrm{m}}}$, the new velocity in the center of mass system is modified by adding to it an incremental velocity $\overrightarrow{\Delta V}$ is chosen by random selection from one of the probability distribution tables stored in memory for this purpose. An index associated with the scatterer determines which table is to be used. The direction cosines of $\vec{\Delta} \mathrm{V}, \alpha^{\prime \prime \prime}, \beta^{\prime \prime \prime}$, and $\gamma^{\prime \prime \prime}$, are selected at random from an isotropic distribution and finally the emergent velocity is 


$$
\begin{aligned}
& \mathrm{v}_{\mathrm{e}}=\left\{\Delta \mathrm{v}^{2}+2 \frac{\mathrm{v} \Delta \mathrm{v}}{a+1}\left[\alpha^{\prime \prime \prime}\left(a \alpha^{\prime \prime}+\alpha\right)+\beta^{\prime \prime \prime}\left(a \beta^{\prime \prime}+\beta\right)+\gamma^{\prime \prime}\left(a \gamma^{\prime \prime}+\gamma\right)\right]\right. \\
& \left.+\left[a^{2}+1+2 a\left(\alpha \alpha^{\prime \prime}+\beta \beta^{\prime \prime}+\gamma \gamma^{\prime \prime}\right)\right] \frac{\mathrm{v}^{2}}{(a+1)^{2}}\right]^{\frac{1}{2}} \\
& \alpha_{\mathrm{e}}=\frac{\frac{\mathrm{v}}{a+1}\left(a \alpha^{\prime \prime}+\alpha\right)+\Delta \mathrm{V} \alpha^{\prime \prime \prime}}{\mathrm{v}_{\mathrm{e}}} \\
& \beta_{\mathrm{e}}=\frac{\frac{\mathrm{v}}{a+1}\left(a \beta^{\prime \prime}+\beta\right)+\Delta \mathrm{V} \beta^{\prime \prime \prime}}{\mathrm{v}_{\mathrm{e}}} \\
& \gamma_{\mathrm{e}}=\frac{\frac{\mathrm{v}}{a+1}\left(a \gamma^{\prime \prime}+\gamma\right)+\Delta \mathrm{v} \gamma^{\prime \prime \prime}}{\mathrm{v}_{\mathrm{e}}}
\end{aligned}
$$

When moderation is not significant, (44) reduces to a simpler set of equations.

\section{Inelastic Scattering}

Inelastic scattering in this program is assumed to be isotropic in the center of mass system. Although this may not always be the case in nature, neither the experimental data nor the importance of the effect justify including anisotropic inelastic scattering in the program at this time. Should the situation change in the future, the appropriate modifications can easily be made.

The emergent velocity in the center of mass system is chosen from either the evaporation model spectrum or one of the probability distribution tables stored in the memory for this purpose. The program determines whether to use the evaporation model or, if not, which table to use by the value of number, $s$, which is part of the data stored with each isotope in the basic library of data. If $S^{\prime}$ is negative, it is interpreted as the name of the inelastic spectrum table; otherwise, it is interpreted as the resonance spacing to be used in the evaporation model. 
Using the evaporation model, the emergent velocity, $V^{\prime}$, is chosen from

$$
\begin{aligned}
& \frac{\mathrm{dp}\left(\mathrm{V}^{\prime}\right)}{\mathrm{dV}^{2}}=\mathrm{CV} \mathrm{\prime}^{2} \mathrm{e}^{-\mathrm{V}^{\prime 2} / \mathrm{T}_{\mathrm{N}}} \\
& \mathrm{T}_{\mathrm{N}} \equiv \mathrm{DV}
\end{aligned}
$$

$\mathrm{V}$ is the incident velocity of the neutron and $\mathrm{C}$ is a normalization constant.

The method of selecting from this distribution is described in Section XIII A.

When the inelastic spectrum is determined by one of the probability distribution tables, the method is to compare a random number $\xi$ successively with the probabilities $P_{i}$, of the table to find the pair for which

$$
P_{i}>\xi \geq P_{i+1}
$$

The emergent velocity in the center of mass system is then

$$
v^{\prime \prime}=\left(v^{2}-v_{i}^{2}\right)^{\frac{1}{2}}
$$

provided the value so obtained is positive. To assure that it will be, $\xi$ is drawn from a. (uniform) distribution between zero and the entry, $\mathrm{p}_{\mathrm{j}}$, in the probability table such that

$$
\mathrm{v}_{\mathrm{j}}^{2} \leq \mathrm{v}^{2}<\mathrm{v}_{\mathrm{j}-1}^{2}
$$

$p_{i}$ and $v_{j}^{2}$ that corresponding to $p_{j}$. In this way the table is used to select an energy loss (proportional to $v_{i}^{2}$ ) according to the inelastic $\gamma$ ray spectrum. In the event that the incident velocity is less than the smallest $v_{i}$, that is if $v^{2}<v_{17}^{2}$ no inelastically scattered particle is permitted to emerge.

If the atomic weight of the scatterer is high, that is if

$$
a>a_{\mathrm{h}}
$$

the center of mass velocity and direction is used for the laboratory system values.

Otherwise the laboratory system values $\mathrm{V}^{\prime}, \alpha^{\prime}, \beta^{\prime}, \gamma^{\prime}$ are computed by adding vectorially the velocity of the center of mass system. 


$$
\begin{aligned}
& V^{\prime}=\left[V^{\prime \prime}+v^{2}\left(\frac{1}{a+1}\right)^{2}+2 V^{\prime \prime} V \frac{\alpha \alpha^{\prime \prime}+\beta \beta^{\prime \prime}+\gamma \gamma^{\prime \prime}}{a+1}\right]^{1 / 2} \\
& \alpha^{\prime}=\left(V^{\prime \prime} \alpha+\frac{V \alpha}{1+a}\right) / V^{\prime} \\
& \beta^{\prime}=\left(V^{\prime \prime} \beta^{\prime \prime}+\frac{V \beta}{1+a}\right) / V^{\prime} \\
& \gamma^{\prime}=\left(V^{\prime \prime} \gamma^{\prime \prime}+\frac{v \gamma}{1+a}\right) / V^{\prime}
\end{aligned}
$$

and (47) and (48) are modified to

$$
v^{\prime \prime}=\left[v^{2}\left|\frac{a}{1+a}\right|^{2}-v_{i}^{2}\right]^{1 / 2} \text { and } v_{j}^{2} \leq v^{2} \cdot\left(\frac{a}{a+1}\right)^{2}<v_{j}^{2}-1
$$

Since it is assumed that the evaporation model spectrum will only be used for heavy nuclei, no provision is made for using the center of mass system incident velocity in (45). The use of the inelastic spectrum tables, implies the approximation has been made that the spectrum of $\gamma$ particles emitted is independent of the incident energy except that it has been truncated to prevent the emission of neutrons at negative energies.

\section{Fission}

Fission neutrons are assumed to be isotropically emitted. The emergent velocity is obtained by random selection from the fission spectrum table, $\left(p_{n}, v_{n}^{2}\right)$. A random number, $\xi$, is compared with the successive $p_{n}$ to obtain an $n$ such that

$$
\mathrm{p}_{\mathrm{n}}>\xi \geq \mathrm{p}_{\mathrm{n}+1}
$$

Then

$$
v^{2}=v_{n}^{2}+\frac{\xi^{-p} p_{n}}{p_{n+1}-p_{n}}\left(v_{n+1}^{2}-v_{n}^{2}\right)
$$

Thus $\mathrm{V}$ is obtained by linear interpolation in the square of $\mathrm{V}$ from the fission spectrum tables. 


\section{Census Time}

For those particles for which $S_{T}$, the distance to census time, is less than $S_{c}$ or $S_{b}$ the coordinates are advanced to the position at which $t=T$. That is

$$
\begin{aligned}
& \mathrm{x}+\alpha\left(\frac{\mathrm{T}}{\delta}\right) \rightarrow \mathrm{x} \\
& \mathrm{y}+\beta\left(\frac{\mathrm{T}}{\delta}\right) \rightarrow \mathrm{y} \\
& \mathrm{z}+\gamma\left(\frac{\mathrm{T}}{\delta}\right) \rightarrow \mathrm{z} \\
& 0 \rightarrow \mathrm{t}
\end{aligned}
$$

and the new coordinates are written. on the census tape with a value of $\mathrm{N}$ equal to the number of particles of the set for which $S_{T}<\left(S_{c}, S_{b}\right)$. In (52) $\delta$ is a number slightly greater than unity used whenever a particle is advanced to a new position to assure that round off errors do not place the particle in a different region from the anticipated one.

\section{J. Reflection at Outer Boundaries}

When a particle is advanced, in the between collisions routine, to an outer boundary of the system it may be reflected back into the system. The reflection coefficient or albedo, $\omega_{b j}$, a function of boundary and of macroscopic group, may have any positive value. It is computed from the macroscopic fluxes by Equation (63). On reflection, the

value of $\pi$, the intensity of the expectation beam is multiplied by $\omega_{b j}$, while the number of reflected particles, $\mathrm{N}^{\prime}$, is obtained from the incident number, $\mathrm{N}$, by

$$
\mathrm{N}^{\prime}=f \mathrm{~N} \omega_{b j}+\xi \neq
$$

The direction cosines $\alpha^{\prime}, \beta^{\prime}, \gamma^{\prime}$ are computed from the relations

$$
\begin{aligned}
& \alpha^{\prime}=\alpha-2 \lambda \frac{\Delta}{\alpha} \\
& \beta^{\prime}=\beta-2 \delta \frac{\Delta}{\beta} . \\
& \gamma^{\prime}=\gamma-2 \rho \frac{\Delta}{\gamma}
\end{aligned}
$$

where $\chi, \delta, \rho$, and $\Delta$ are defined by (31) and (32) or (33) with $x, y, z$, the point at which the particle hits the boundary and $\dot{\alpha}, \beta$, and $\gamma$ the incident direction. 


\section{MONTE CARLO CONSTANTS}

Storage assignments, geometric data, etc., for the constants for a monte carlo calculation on a system do not change during the course of calculation so that all such quantities are computed only once for each monte carlo system just preceding the first monte carlo time step. The quantities which do change with time are the acceleration factors, $\mathrm{A}_{\mathrm{ih}}^{\mathrm{t}}$; and the quantities dependent on the isotope concentrations in each material, that is, the concentrations themselves which are used in the resonance calculation; the mean free paths, $\lambda_{\mathrm{mi}}$; the collision neutron emission numbers, $\mathrm{P}_{\mathrm{mi}}, \mathrm{P}_{\mathrm{mi}}{ }_{\text {inel }}$, and $\mathrm{P}_{\mathrm{mi}}$; and the anisotropic scattering coefficients, $\mathrm{a}_{0_{\mathrm{rmi}}}, \mathrm{a}_{1} \mathrm{rmi}, \mathrm{a}_{2} \mathrm{rmi}$, and $\mathrm{a}_{3}$ for the heavy scatterers which are actually combinations of the heavy scattering isotopes. in a material. The equations by which these are calculated are

$$
\begin{aligned}
& \lambda_{\mathrm{mi}}=\left[\sum_{\mathrm{a}} \mathrm{m}_{\mathrm{am}}^{\mathrm{N}_{\mathrm{ai}} \times 10^{-24}}\right]^{-1} \\
& \mathrm{P}_{\mathrm{mi}}=\lambda_{\mathrm{mi}} \sum^{\prime} \mathrm{N}_{\mathrm{am}}\left(\nu_{\text {inel }} \sigma_{\text {inel }{ }_{\mathrm{ai}}} \times 10^{-24}\right.
\end{aligned}
$$

where the primed sum indicates all isotopes in $m$ having the same value of $S^{\prime}$, the inelastic spectrum indication. There will be $\mathrm{R}_{\mathrm{m}}^{\prime}$ of the $\mathrm{P}_{\mathrm{mi}}{ }_{\text {inel }}$ for group $\mathrm{i}$ region $\mathrm{m}$.

$$
\begin{gathered}
\mathrm{P}_{\mathrm{mi}_{\mathrm{f}}}=\lambda_{\mathrm{mi}} \sum_{\mathrm{in}} \mathrm{m}_{\mathrm{am}}\left(\nu_{\mathrm{f}} \sigma_{\mathrm{f}}\right)_{\mathrm{ai}} \times 10^{-24} \\
\mathrm{P}_{\mathrm{mi}}=\lambda_{\mathrm{mi}} \sum^{\prime \prime} \mathrm{N}_{\mathrm{am}} \sigma_{\mathrm{el}} \times 10^{-24}
\end{gathered}
$$

for heavy isotopes.

$$
\mathrm{P}_{\mathrm{mi}}=\lambda_{\mathrm{mi}} \mathrm{N}_{\mathrm{am}} \sigma_{\mathrm{el}} \times 10^{-24}
$$

for light isotopes. 
The doubly primed sum indicates all isotopes in $\mathrm{m}$ having atomic weight greater than $a_{h}$, an input constant. These are heavy isotopes, moderation by which is assumed to be unimportant except in the resonance range. For scattering by these isotopes outside the range $\mathrm{I}_{\mathrm{L}_{\mathrm{m}}}>\mathrm{i} \geq \mathrm{I}_{\mathrm{m}}$ moderation is neglected; within that range a constant atomic weight $a_{h}$ is used. For these isotopes the anisotropic scattering coefficients must be averaged:

$$
a_{{ }_{\mathrm{mi}}}=\left[\sum^{\prime \prime} \mathrm{N}_{\mathrm{am}} \sigma_{\mathrm{ai}}{ }_{\mathrm{el}}{ }^{a_{\ell}}\right] / \sum_{\mathrm{ai}}^{\prime \prime} \mathrm{N}_{\mathrm{am}}{ }^{\sigma_{\mathrm{ai}}}
$$

The calculation of $a_{m}$ is made as follows:

Let

$$
a(\mu)=a_{0}+a_{1} \mu+a_{2} \mu^{2}+a_{3} \mu^{3}
$$

Two cases may be distinguished, the cubic and quadratic forms:

$$
\begin{aligned}
& \text { 1. } a_{3} \neq 0 \\
& a_{2}^{2}-3 a_{1} a_{3} \geq 0 \\
& a_{m}=\max [a(-1), a(1), a(\mu)] \\
& \text { where } \mu_{-}=\frac{-a_{2}-\sqrt{a_{2}^{2}-3 a_{1} a_{3}}}{3 a_{3}} \\
& \text { If } a_{2}^{2}-3 a_{1}, a_{3}<0 \\
& a_{m}=\max [a(-1), a(1)] \\
& 2 . a_{3}=0 \\
& \text { If } a_{2} \neq 0 \\
& a_{m}=\max \left[a(-1), a(1), a\left(\mu_{0}\right)\right]
\end{aligned}
$$


where $\mu_{0}=-\frac{a_{1}}{2 a_{2}}$

If $\mathrm{a}_{2}=0$ (the linear form)

$$
a_{m}=\max [a(-1), a(1)]
$$

The acceleration factors $A_{\text {if }}^{t}$ are obtained for the third and subsequent monte carlo step flux tallies as the ratio of the flux in group $i$, region $h$ in the last monte carlo step to that in the one before

$$
A_{\text {ih }}^{t}=\frac{\phi_{\text {if }}^{t-1}}{\phi_{\text {if }}^{t-2}}
$$

The albedoes are obtained from the diffusion theory fluxes. Albedoes are nonzero at interfaces between monte carlo systems.

For each system, $\mathrm{s}$, the index, $\mathrm{K}_{\mathrm{s}}$, of the highest macroscopic region number in the system is specified in the input. Albedoes for the right (outer) boundary of a system are computed by means of equation (63) from the diffusion fluxes at the system boundaries, $\mathrm{N}_{\mathrm{K}}$, for $\mathrm{K}=\mathrm{K}_{\mathrm{S}}$. Albedoes at the left boundary are obtained as the reciprocals of the corresponding values for the preceding system. $M$ is a correction factor supplied in the input for each boundary of each system ( 2 per system). For the left boundary of system 1 and the right boundary of system $S^{\prime}$, the albedoes are obtained by substituting equation (69) into equation (63) and solving for the $\omega_{j}$ in terms of the $\mathrm{B}_{\mathrm{o}_{j}}$ and $\mathrm{B}_{\mathrm{N}_{j}}$.

$$
\omega_{j}=\frac{\phi_{N_{k}}+2 D_{k}\left[\frac{\phi_{k}{ }^{j-\phi} N_{k}-1 j}{\Delta r_{k}}\right]}{\phi_{N_{k}}-2 D_{k}\left[\frac{\phi_{k}{ }_{k}^{j-\phi} N_{k}-1, j}{\Delta r_{k}}\right]} M
$$




$$
\begin{gathered}
\omega_{o_{j}}=\frac{1-2 \frac{{ }_{o_{j}}}{1-B_{o_{j}}}}{1+2 \frac{{ }_{o_{j}}}{1-B_{o_{j}}}} M_{1-} \\
c_{N_{j}}=\frac{1+2 \frac{B_{N}}{1-B_{N}}}{1-2 \frac{B_{N_{j}}}{1-B_{N_{j}}}} M_{k+}
\end{gathered}
$$

In equation (63), $\mathrm{N}_{\mathrm{k}}$ is the mesh point at the boundary separating region $\mathrm{k}$ and region $\mathrm{k}+1$, and $\mathrm{D}_{\mathrm{k}}$ and $\Delta \mathrm{r}_{\mathrm{k}}$ are respectively the diffusion coefficients and mesh spacing in region k. In equation (63), indices referring to the boundary and to the macroscopic energy group have been suppressed. For each boundary between two systems, then, an albedo is computed for each macroscopic group for each of the two systems. Since the RBU Program begins with a monte carlo calculation performed before the diffusion fluxes are available, it is necessary to input a set of albedoes for the first monte carlo steps. 


\section{THE DIFFUSION CALCULATION}

As was discussed in the first section of this report, most of the neutron flux and reactivity calculations in $\mathrm{RBU}$ are made using the diffusion approximation. The fluxes and other quantities obtained from the monte carlo portion of the program are used to obtain constants for a one space dimensional diffusion calculation. In this, the spatial variable, r, may be either a cartesian coordinate, the radial variable of a set of cylindrical coordinates or the radial variable of a set of spherical coordinates. Its range is divided into a set of discrete points, $R_{0} \leq r_{n} \leq R_{N^{*}}$. The macroscopic region boundaries, as represented in this one dimensional model form a subset $R_{k}$ of the $r_{n}$. Within any macroscopic region, the mesh spacing and the nuclear properties of the system are assumed independent of $r$.

The second independent variable of the diffusion calculation is the neutron energy, $\mathrm{E}$. The range of this variable, also, is represented by a set of discrete values $E_{J} \leq E_{j} \leq E_{0}$ which correspond to the limits of the macroscopic groups discussed earlier.

In terms of the continuous variables, $r$ and $E$, the diffusion equation is $\nabla\left[\mathrm{D}(\mathrm{r}, \mathrm{E}) \nabla_{\phi}(\mathrm{r}, \mathrm{E})\right]-\Sigma_{\mathrm{t}}(\mathrm{r}, \mathrm{E}) \phi(\mathrm{r}, \mathrm{E})$ $+\chi_{i n}(r, E) \int_{E}^{E} \Sigma_{i n}\left(r, E^{\prime}\right)\left(\nu_{i n} r, E^{\prime}\right) \phi\left(r, E^{\prime}\right) \frac{d E^{\prime}}{\int_{0}^{E^{\prime}} \chi_{i n}\left(r, E^{\prime \prime}\right) d E^{\prime \prime}}$

$$
\begin{aligned}
& +\sum_{A} \int_{E}^{\min \left(E / \alpha_{A}, E_{o}\right)} \frac{\Sigma_{S_{A}}\left(r, E^{\prime}\right) \phi\left(r, E^{\prime}\right) d^{\prime}}{\left(1-\alpha_{A}\right) E^{\prime}} \\
& =\frac{-x_{f}}{k} \int_{E_{J}}^{E_{o}} \Sigma_{f}\left(r, E^{\prime}\right) \nu_{f}\left(r, E^{\prime}\right) \phi\left(r, E^{\prime}\right) d E^{\prime}
\end{aligned}
$$


The usual approximations namely that $\chi_{\text {in }}$ is independent of the incident energy except for truncation and renormalization at that value and that elastic scattering is isotropic in the center of mass system have been made in writing (64).

Since the properties of a diffusion calculation on a thermal or intermediate reactor are not very sensitive to the details of the inelastic scattering spectrum and since experimental data does not warrant more detail, the first of these appears perfectly satisfactory. The second approximation also should introduce no detectable error in most calculations; in a sense the use of diffusion theory implies this. Should it become significant in special cases, however, minor changes in the pre-diffusion portion of the program or use of a set of synthetic scatterers can eliminate its effect on the third term of equation (64). No such assumption is involved in the calculation of $D$, which is obtained directly from the monte carlo.

$$
\left.\begin{array}{l}
-\nabla\left[D_{j}(r) \nabla \cdot \phi_{j}(r)\right]+T_{j}(r) \phi_{j}(r)=H_{j}(r) \\
H_{j}(r)=\sum_{j^{\prime}=1}^{j-1} T_{j j^{\prime}}(r) \phi_{j^{\prime}}(r)+\chi_{j} P(r) \\
P(r)=\frac{1}{k} \sum_{j^{\prime}=1}^{J} F_{j^{\prime}}(r) \phi_{j^{\prime}}(r)
\end{array}\right\} .
$$

The nuclear constants appearing in (65a) and $(65 \mathrm{~b})$ are defined in the section of this report on Diffusion Constants.

\section{A. The Difference Equations}

With the discrete spatial mesh equation (65) becomes, on dropping the subscript $\mathrm{j}$ :

$$
-a_{n} \phi_{n-1}+b_{n} \phi_{n}-c_{n} \phi_{n+1}-d_{n}=0
$$


where

$$
\begin{aligned}
& a_{n}=\left(D_{n-}\right) \frac{r_{n-1 / 2}^{\rho}}{\Delta r_{n-}} \\
& b_{n}=a_{n}+c_{n}+\left(T_{-n-}\right) r_{n}^{\rho} \frac{\Delta r_{n-}}{2}+\left(T_{n+}\right) r_{n}^{\rho} \frac{\Delta r_{n+}}{2} \\
& c_{n}=\left(D_{n+}\right) \frac{r_{n+1 / 2}^{\rho}}{\Delta r_{n+}} \\
& d_{n}=r_{n}^{\rho} \frac{\Delta r_{n-}}{2}+r_{n-}+r_{n}^{\rho} \frac{\Delta r_{n+}}{2} H_{n+} . \\
& H_{n-}=\sum_{j^{\prime}=1}^{j-1} T_{n-, j j^{\prime}} \phi_{n j^{\prime}}+\chi_{j} P_{n-} ; H_{n+}=\sum_{j^{\prime}=1} T_{n+, j j^{\prime}} \phi_{n j^{\prime}}+\chi_{j} P_{n+}
\end{aligned}
$$

where

$$
\begin{aligned}
& \rho \quad=0,1 \text {, or } 2 \text { for plane, cylindrical, or spherical geometries respectively } \\
& \Delta \mathrm{r}_{\mathrm{n}+}=\mathrm{r}_{\mathrm{n}+1}-\mathrm{r}_{\mathrm{n}} \\
& \mathrm{r}_{\mathrm{n}-}=\mathrm{r}_{\mathrm{n}}-\mathrm{r}_{\mathrm{n}-1}
\end{aligned}
$$

In (66), (67), and (68), minus signs associated with an index, $n$, indicate values of mesh spacing, power, and nuclear constants appropriate where $r<r_{n}$ of mesh point $n$ while plus signs indicate those appropriate where $r>r_{n}$. When $r_{n}$ does not coincide with a region boundairy, $\mathrm{R}_{\mathrm{k}},(67)$ and $(68)$ reduce to

$$
\begin{aligned}
& a_{n}=D_{n} \frac{r_{n-1 / 2}^{\rho}}{\Delta r_{n}} \\
& b_{n}=a_{n}+c_{n}+T_{n} r_{n}^{\rho} \Delta r_{n} \\
& c_{n}=D_{n} \frac{r_{n+1 / 2}^{\rho}}{\Delta r_{n}} \\
& d_{n}=r_{n}^{\rho} \Delta r_{n} H_{n} \cdots
\end{aligned}
$$




$$
H_{n}=\sum_{j^{\prime}=1}^{j-1} T_{n, j j^{\prime}} \phi_{n j^{\prime}}+\chi_{j} P_{n}
$$

At the boundaries of the mesh, $R_{0}$ and $R_{N}$, the equations for $a_{n_{j}}, b_{n, j}, c_{n, j}$, and $d_{n, j}$ are modified to agree with the boundary conditions,

$$
\begin{aligned}
& -\left(1-B_{0, j}\right) D_{0, j} \frac{\phi_{1, j}-\phi_{0, j}}{\Delta r_{0+}}+B_{0, j} \phi_{0, j}=0 \\
& \left(1-B_{N, j}\right) D_{N, j} \frac{\phi_{N, j}-\phi_{N-1, j}}{\Delta r_{N}-}+B_{N, j} \phi_{N, j}=0
\end{aligned}
$$

which correspond to the physical boundary conditions

$$
\begin{aligned}
& -\left[1-\mathrm{B}_{0}(\mathrm{E})\right] \mathrm{D}\left(\mathrm{R}_{0}, \mathrm{E}\right) \nabla \phi\left(\mathrm{R}_{0}, \mathrm{E}\right)+\mathrm{B}_{0}(\mathrm{E}) \phi(\mathrm{R}, \mathrm{E})=0 \\
& {\left[1-\mathrm{B}_{\mathrm{N}}(\mathrm{E})\right] \mathrm{D}\left(\mathrm{R}_{\mathrm{N}}, \mathrm{E}\right) \nabla \phi\left(\mathrm{R}_{N}, \mathrm{E}\right)+\mathrm{B}_{\mathrm{N}}(\mathrm{E}) \phi\left(\mathrm{R}_{N}, \mathrm{E}\right)=0}
\end{aligned}
$$

When used in $R B U$, it is generally true that $R_{0}=0, B_{0}(E)=0, B_{N}(E)=0.3194$, the generality of (70) is included in the program to make the use of the diffusion portion without the remainder of RBU convenient.

Equation (69) leads to the following equations for $n=0$

$$
\begin{aligned}
& a_{0, j}=0 \\
& b_{0, j}=c_{0, j}+B_{0, j} \Delta r_{0+} \\
& c_{0, j}=\left(1-B_{0, j}\right) D_{0} \\
& d_{0, j}=0
\end{aligned}
$$

and for $\mathrm{n}=\mathrm{N}$

$$
\begin{aligned}
& a_{N, j}=\left(1-B_{N, j}\right) D_{N, j} \\
& b_{N, j}=a_{N, j}+B_{N, j} \Delta r_{N-}
\end{aligned}
$$

* In order to obtain the correct variation of the extrapolation length with energy in the diffusion calculation, one uses the actual physical radius of the reactor for the diffusion mesh and allows the extrapolation distance $0.7104 \lambda$ to be accounted for by the value of ${ }^{\mathrm{B} j}$. 


$$
\begin{aligned}
& c_{N, j}=0 \\
& d_{N, j}=0
\end{aligned}
$$

\section{B. Numerical Method}

The spatial integration subject simultaneously to boundary conditions at both ends of the mesh is performed with the use of auxiliary functions $p_{n, j}$ and $q_{n, j}$. Defining these by

$$
\phi_{n, j}=p_{n, j} \phi_{n+1, j}+q_{n, j}
$$

leads to

$$
\begin{aligned}
& p_{n, j}=\frac{c_{n, j}}{b_{n, j}-a_{n, j} p_{n-1, j}} \\
& q_{n, j}=\frac{d_{n, j}+a_{n, j} q_{n-1, j}}{b_{n, j}-a_{n} j^{p_{n-1, j}}} \\
& p_{-1, j}=q_{-1, j}=0
\end{aligned}
$$

A process of finding a set of $\phi_{n, j}$ which satisfy equations (66) and (69) when a set of $P_{n}$ is known is the following. The $H_{n, 1}$ are computed using (68) from $x_{1}$, and $P_{n}$. From these, using (67), (68), (71), and (72) the $a_{n, 1}, b_{n, 1}, c_{n, 1}$, and $d_{n, 1}$ are computed. The $p_{n, 1}$ and $q_{n, 1}$ may then be computed using equation (74) successively for $n=0$, $\mathrm{n}=1, \ldots \mathrm{n}=\mathrm{N}$. Beginning now with $\mathrm{n}=\mathrm{N}$, the $\phi_{\mathrm{n}, 1}$ are computed with equation (7.3). Note that by (72) and (74), $\mathrm{d}_{\mathrm{N} 1}$ may be computed without any assumption about ${ }^{(} \phi_{\mathrm{N}+1,1}$ ). With the $\phi_{\mathrm{n}, 1}, \chi_{2}$, and $\mathrm{P}_{\mathrm{n}}$, the $\mathrm{H}_{\mathrm{n}, 2}$ are now computed and the process above repeated for $j=2$. Continuing in this fashion, all the $\phi_{n, j}$ are found.

The $\phi_{n, j}$ may be used to compute a set of $P_{n}$ from the discretized version of $(65 b)$

$$
P_{n}=\frac{1}{k} \sum_{j^{\prime}=1}^{J} F_{n j^{\prime}} \phi_{n j^{\prime}}
$$

where $k$, the multiplication, is defined by

$$
h \equiv \frac{1}{V} \sum_{n=0}^{N} \sum_{j^{\prime}=1}^{J}\left[F_{n-j^{\prime}} \Delta r_{n-}+F_{n+, j^{\prime}} \Delta r_{n+}\right] \phi_{n, j^{\prime}} \frac{r_{n}}{-41-}
$$


so that

$$
\begin{aligned}
& \sum_{n=0}^{N} P_{n} \Delta V_{n}=V \\
& \left.V=\sum_{n=0}^{N} r_{n}^{\rho} \frac{\Delta r_{n-}}{2}+\frac{\Delta r_{n+}}{2}\right) \\
& \Delta r_{o-} \equiv \Delta r_{N+} \equiv P_{o^{-}} \equiv P_{N+} \equiv 0
\end{aligned}
$$

For values of $\mathrm{n}$ in (76) which do not correspond to region boundaries, the terms reduce to $\mathrm{F}_{\mathrm{n}} \phi_{\mathrm{n}} \mathrm{r}_{\mathrm{n}}^{\rho} \Delta \mathrm{r}_{\mathrm{n}}$ and $\mathrm{r}_{\mathrm{n}}^{\rho} \Delta \mathrm{r}_{\mathrm{n}}$.

The $\mathrm{P}_{\mathrm{n}}$ so obtained may be used to repeat the calculation of the $\phi_{\mathrm{jn}}$ and the iterative process continued. Ultimately the values of $k_{,} \phi_{n j}$, and $P_{n}$ found in this way converge to a consistent solution.

\section{Acceleration}

For reactors in which the multiplications of the second and higher modes are only slightly smaller than that of the first, convergence will be slow; for this reason the procedure described above is modified to improve the rate. The modifications used in RBU are made in the calculation of $\mathrm{H}_{n, j}$ in (68) and (68a) and correspond to a combination of the Extrapolated Liebman method and the Aitken $\delta^{2}$ process. The $\phi_{n, j^{\prime}}$ in the first term of $\mathrm{H}_{n, j}$ appear only for $\mathrm{j}^{\prime}<\mathrm{j}$. It is therefore possible to use as was indicated in the preceding description of the process, values obtained in the current iteration, iteration $p$. The $\phi_{n, j}$ occurring in the second term (see equation 75 ) appear for all values of $j^{\prime}$ between 1 and $J$ inclusive some of which are known only for iteration $p-1$. For $j^{\prime}<j$, however, the values for iteration $p$ are available and it is desirable to use them. Inserting the iteration number in (75) with this modification we have for the $\mathbf{P}_{n}^{(p)}$ from which to compute the $\phi_{n, j+1}^{(p)}$

$$
P_{n(j)}^{(p)}=\frac{1}{k(j)}\left[\sum_{j^{\prime}=1}^{j} F_{n j^{\prime}} \phi_{n, j^{\prime}}^{(p)}+\sum_{j^{\prime}=j^{\prime}+1}^{J} F_{n j^{\prime}} \phi_{n, j^{\prime}}^{(p-1)}\right]
$$


The group subscript, $j$, in (77) is placed in parenthesis to indicate that while $P_{n}$ and $k$ are not strictly a function of $j$, the method used here for their evaluation, makes the values vary according to the group being worked on at any stage of the calculation. The value of $h$ appearing in (77) is defined in (76) as before but it must now be re-evaluated at each group.

There is one further modification to be made to obtain the final form of (75). This is to use for $\mathrm{P}_{\mathrm{n}(0)}^{(\mathrm{p}+1)}$ a value which is extrapolated beyond that given by (77) in accordance with our information about $P_{n(0)}^{(p)}$.

$$
\mathrm{P}_{n(0)}^{(\mathrm{p}+1)}=\mathrm{P}_{\mathrm{n}(\mathrm{j})}^{(\mathrm{p})}+\omega \mathrm{P}_{\mathrm{n}(\mathrm{J})}^{(\mathrm{p})}-\mathrm{P}_{\mathrm{n}(0)}^{(\mathrm{p})}
$$

where $\omega$ is an acceleration factor supplied as input and $\mathbf{P}_{n(0)}^{(p)}$ is the source from which the fluxes of the first group for iteration p are computed. Equation (77) implies a complete re-evaluation of $P_{n}$ for each group. Actually, this is not necessary since only the changes in $k$ and $P_{n}$ resulting from the difference $\phi_{n, j}^{(p)}-\phi_{n, j}^{(p-1)}$ need be considered. Thus,

$$
\begin{aligned}
& p_{n(j)}^{(p)}=\frac{1}{h_{(j)}}\left[k_{(j-1)} P_{n(j-1)}+F_{n, j}\left(\phi_{n, j}^{(p)}-\phi_{n, j}^{(p-1)}\right)\right] \\
& h_{(j)}=k_{(j-1)}+\frac{1}{v} \sum_{n}\left(\phi_{n, j}^{(p)}-\phi_{n, j}^{(p-1)}\right)\left(F_{n-j} \Delta r_{n-}+F_{n+j} \Delta r_{n+}\right) \frac{r_{n}^{\rho}}{2}
\end{aligned}
$$

In solving equations of the form $\mathrm{F}(\alpha)=\alpha$ by an iterative procedure of the type

$$
\alpha_{p+1}=F\left(\alpha_{p}\right)
$$

it is possible to estimate the value of the root from the values of three successive iterates by Aitken's $\delta^{2}$ process. The error in the $\mathrm{p}^{\text {th }}$ iterate is

$$
\alpha-\alpha_{p}=F(\alpha)-F\left(\alpha_{p-1}\right)
$$

which may be expanded in a Taylor's series about $\alpha$ :

$$
\alpha-\alpha_{p}=\left(\alpha-\alpha_{p-1}\right) F^{\prime \prime}(\alpha)+\ldots
$$

Provided either that

$$
\alpha-\alpha_{\mathrm{p}} \ll 1 \text { or } \mathrm{F}^{\prime \prime}(\alpha) \ll \mathrm{F}^{\prime}(\alpha)
$$


the higher order terms may be dropped, and $\alpha$ can be determined from the values of three successive $\alpha_{\mathrm{p}}$ :

$$
\begin{aligned}
& \frac{\alpha-\alpha_{\mathrm{p}}}{\alpha-\alpha_{\mathrm{p}-1}}=\frac{\alpha-\alpha_{\mathrm{p}-1}}{\alpha-\alpha_{\mathrm{p}-2}} \\
& \alpha \approx \alpha_{\mathrm{p}}-\frac{\left(\alpha_{\mathrm{p}}-\alpha_{\mathrm{p}-1}\right)^{2}}{\alpha_{\mathrm{p}}-2 \alpha_{\mathrm{p}-1}+\alpha_{\mathrm{p}-2}}
\end{aligned}
$$

It is clear that the process for getting $P_{n}$ described above can be written as a set of coupled equations of the form (81). Equation (82) then takes the form

$$
\alpha_{n}-\alpha_{n}^{(p)}=\sum_{n^{\prime}=1}^{N}\left(\alpha_{n^{\prime}}-\alpha_{n^{\prime}}^{(p)}\right) \frac{\partial F_{n}\left(\alpha_{1}^{(p)}, \alpha_{2}^{(p)} \cdots \alpha_{N}^{(p)}\right)}{\partial \alpha_{n^{\prime}}}
$$

On the assumption that $\frac{\partial F}{d \alpha_{n^{\prime}}}$ is small for $n^{\prime} \neq n,(84)$ appears to be a valid method to estimate $P_{n}$. For this reason after each set of three iterations carried out as described in (79) the set of $P_{n}$ used in (68a) for the next iteration is obtained from (84) rather than from (79). Equation (84), however, involving, as it does, a ratio of differences, may become inaccurate when the successive $\alpha_{p}$ differ only in a few of the least significant digits. For this reason, when the precision of the differences involved becomes less than fifteen bits for a particular mesh point, the program ceases to use the Aitken $\delta^{2}$ process for that mesh point.

\section{Specific Numerical Procedure}

In the following paragraphs, the numerical process of manipulating the $\mathrm{P}_{n}$ and $k$ is outlined in detail. For clarity the discussion begins at a point in the diffusion calculation during the $p^{\text {th }}$ iteration when fluxes for group $j-1$ have been calculated and the multiplication and source corrected to give a value of $k_{(j-1)}^{(p)}$ and a set of $P_{n(j-1)}^{(p)}$ for $n=0+, 1,2 \ldots N_{1}^{-}, N_{1}^{+}, \ldots . N^{-}$. Using the $P_{n(j-1)}^{(p)}$, the fluxes for group $j, \phi_{n, j}^{(p)}$, are found by means of equations (67) through (74). Then 


$$
k_{(j)}^{(p)}=k_{(j-1)}^{(p)}+\frac{1}{V} \sum_{n} \Delta v_{n} F_{n j}\left(\phi_{n, j}^{(p)}-\phi_{n, j}^{(p-1)}\right)
$$

where it is understood that

$$
\Delta V_{n} F_{n j}=\frac{r_{n}^{\rho}}{2}\left[F_{n-j} \Delta r_{n-}+F_{n+j} \Delta r_{n+}\right]
$$

if $n$ corresponds to a region boundary, and is simply $r_{n}^{\rho} F_{n j} \Delta r_{n}$ if $n$ does not correspond to a region boundary. Similarly,

$$
P_{n(j)}^{(p)}=\frac{1}{k_{(j)}^{k(p)}}\left[k_{(j-1)}^{(p)} P_{n(j-1)}^{(p)}+F_{n j}\left(\phi_{n, j}^{(p)}-\phi_{n, j}^{(p-1)}\right)\right]
$$

This process is continued for each group until the $\mathrm{P}_{\mathrm{n}(\mathrm{J})}^{\mathrm{p}}$ and $h_{(\mathrm{J})}^{\mathrm{p}}$ have been found. If

$$
\left|k(\mathrm{p})-k_{(\mathrm{J})}-k_{(\mathrm{J})}^{(\mathrm{p}-1)}\right|>\epsilon_{3} \text { or if, for any } n, \frac{\left|\mathrm{P}_{\mathrm{n}(\mathrm{J})}^{(\mathrm{p})}-\mathrm{P}_{\mathrm{n}(\mathrm{J})}^{(\mathrm{p}-1)}\right|}{\mathrm{P}_{\mathrm{n}(\mathrm{J})}^{\mathrm{p}} .}>\epsilon_{4}
$$

another iteration is to be carried out. If three successive iterates obtained by (88) are not available, then the next iteration is similar to that just described but, before beginning it, the latest sources are accelerated.

$$
\begin{aligned}
& P_{n(0)}^{(p+1)}=P_{n(J)}^{(p)}+\omega\left(P_{n(J)}^{(p)}-P_{n(0)}^{(p)}\right) \\
& h_{(0)}^{(p+1)}=k_{(J)}^{(p)}
\end{aligned}
$$

and the $\phi_{\mathrm{n}, \mathrm{j}}^{(\mathrm{p})}$ are replaced by

$$
\phi_{n, j}^{(p)}+\omega\left(\phi_{n, j}^{(p)}-\frac{h_{(j)}^{(j)}}{h_{2}^{(p)}} \phi_{n, j}^{(p-1)}\right) \rightarrow \phi_{n, j}^{(p)}
$$

If the use of equation (90) and (91) leads to any negative $P_{n}$ or $\phi_{n j}$; then the treatment by $(90)$ and (91) for all mesh points is bypassed for that time step. With the $P_{n(0)}^{(p+1)}$ a set of $\phi_{n, 1}^{(p+1)}$ are found. Equations (86) and (88) may then be solved and the process continued. 
If three set of $\mathrm{P}_{\mathrm{n}(0)}$ which were obtained from (88), are available the Aitken $\delta^{2}$ process is used to recompute the $\mathrm{P}_{n(0)}^{(\mathrm{p}+1)}$. In this case $\mathrm{P}_{n(0)}^{(\mathrm{p}+1)}$ is computed by (90) as before but

$$
P_{n}^{\left(p+1^{\prime}\right)}=P_{n(0)}^{(p+1)}-\frac{\left(P_{n(0)}^{(p+1)}-P_{n(0)}^{(p)}\right)^{2}}{P_{n(0)}^{(p+1)}-2 P_{n(0)}^{(p)}+P_{n(0)}^{(p-1)}}
$$

is computed for those $\mathrm{n}$ for which the differences in the numerator and denominator of (93) have fifteen or more significant bits. For values of $n$ for which this is not the case $P_{n}^{\left(p+1^{\prime}\right)}=P_{n(0)}^{(p+1)}$ as obtained from $(90)$. Then the $P_{n(0)}^{(p+1)}$ are replaced by

$$
\frac{V P_{n}^{\left(p+1^{\prime}\right)}}{\sum_{n^{\prime}} \Delta v_{n^{\prime}} P_{n^{\prime}}^{\left(p^{\prime}+1^{\prime}\right)}} \rightarrow P_{n(0)}^{(p+1)}
$$

(If the sign of $\left(\mathrm{P}_{\mathrm{n}(0)}^{(\mathrm{p}+1)}-\mathrm{P}_{\mathrm{n}(0)}^{(\mathrm{p})}\right.$ is the same as the sign of $\mathrm{P}_{\mathrm{n}(0)}^{(\mathrm{p}+1)}-2 \mathrm{P}_{\mathrm{n}(0)}^{(\mathrm{p})}+\mathrm{P}_{\mathrm{n}(0)}^{\mathrm{p}-1}$ the Aitken $\delta^{2}$ process is bypassed. As can easily be seen from equation (92), using the $\delta^{2}$ acceleration in this case would introduce oscillations.) Unfortunately, this set of sources bears no simple relationship to any available set of fluxes so that the groupwise correction procedure of (86) and (88) may not be used. Instead the same set of sources, that computed in (93), is used to calculate the fluxes $\phi_{n, j}^{(p+1)}$ for all $j$. When the $\phi_{n, j}^{(p+1)}$ have been computed, a new multiplication and set of sources is computed from

$$
k_{(0)}^{(p+2)}=\frac{1}{v} \sum_{n} \Delta v_{n} \sum_{j} F_{n j} \phi_{n, j}^{(p+1)}
$$

and

$$
P_{n(0)}^{(p+2)}=\frac{1}{k_{(1)}^{(p+2)}} \sum_{j} F_{n j} \phi_{n, j}^{(p+1)}
$$

Iteration $p+2$ then proceeds by the use of (86) and (88). 
At any time step other than the first, the program begins with a set of $P_{n}$ which was the result of the last iteration performed on the previous time step. The new fluxes, multiplication and sources are then computed in the same way as that used following the Aitken extrapolation, that is, without the groupwise correction procedure. In the first time step, these sources are taken as flat and equal to unity. 


\section{THE DIFFUSION CONSTANTS}

The nuclear constants of Equation (65) are, in general, weighted averages of the microscopic group and region constants used in the monte carlo portion of the program with the monte carlo fluxes used as the weight functions. The exceptions to this are the diffusion coefficients $D_{j k}$, the perpendicular leakage cross section, and, in addition, all constants whose equivalent, in the monte carlo portion are computed from Breit Wigner resonance functions.

For those macroscopic groups and regions not encompassing a resonance range in any of its microscopic groups and regions, a typical macroscopic group average cross section is

$$
\Sigma_{j m p}=\sum_{a \text { in } m} N_{a_{m}} \sigma_{a j m p} \times 10^{-24}
$$

where

$$
\begin{aligned}
& \sigma_{\text {ajmp }}=\sum_{i \text { in } j}\left[\sigma_{\text {aip }} \phi_{i m}\right] / \sum_{\substack{i \text { in } j \\
m^{\prime} \text { in } k_{m}}} \phi_{i ı n^{\prime}} \\
& \phi_{\text {im }}=\sum_{\text {f containing } m} \phi_{\text {if }}
\end{aligned}
$$

The subscript a refers to a particular isotope, $\mathrm{p}$ specifies a cross section type and $\mathrm{m}$ is the index of the material contained in region $h$, a region of region set $f$. The summation over all $f$ containing $m$ to obtain quantities characteristic of a macroscopic region in this and several other equations, and for that matter the whole idea of burning up materials. with macroscopic fluxes, implies thateach material can appear in only one macroscopic region. Although material $\mathrm{m}$ in region $\mathrm{k}$ may initially be physically identical with material $\mathrm{m}^{\prime}$ in region $\mathrm{k}^{\prime}$, that will no longer be true after a few burn up steps. For this reason, the two must have different $m$ indices in the calculation. 
The average over the microscopic region sets in a macroscopic region is

$$
\Sigma_{\mathrm{jkp}}=\sum_{\mathrm{m} \text { in } \mathrm{k}}\left[\Sigma_{\mathrm{jmp}} \phi_{\mathrm{jm}}\right]
$$

The calculations corresponding to (96) are made after each monte carlo step while those corresponding to (95) and (97) are made after each burnup step and each monte carlo step.

The calculations corresponding to (96) are specifically

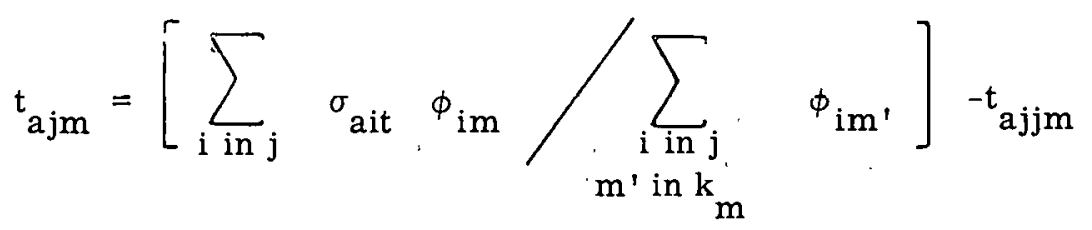

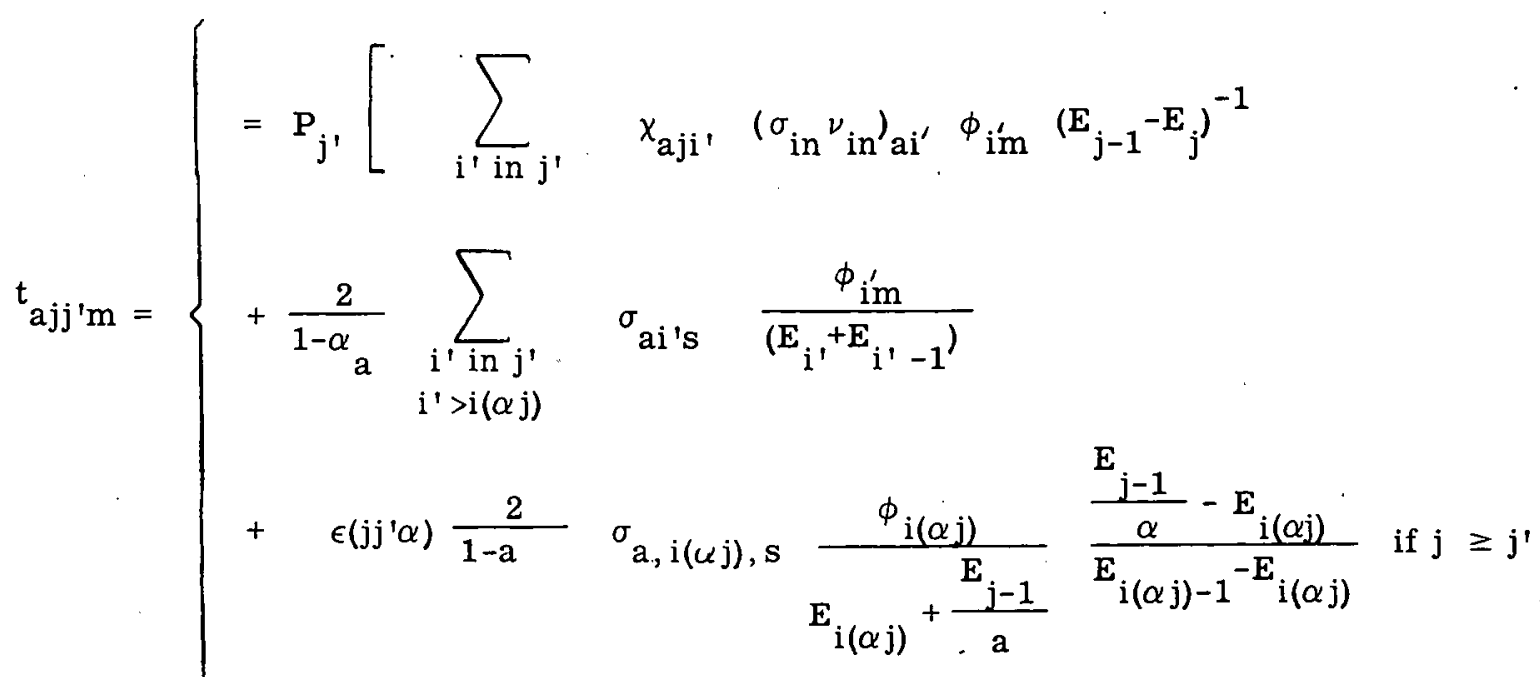

$$
\begin{aligned}
& =0, \quad \text { if } j<j \text {, }
\end{aligned}
$$

where $\mathrm{k}_{\mathrm{m}}$ is the region containing $\mathrm{m}$.

In Equation $(99)$ i $(\alpha ; j)$ is the group such that

$$
E_{i(\alpha j)}<\frac{E_{j-1}}{\alpha} \leq E_{i(\alpha j)-1}
$$


while

$$
\begin{aligned}
\epsilon_{\left(j^{\prime} \alpha\right)} & =0 \text { if } \frac{E_{j-1}}{\alpha} \geq E_{j^{\prime}-1} \\
& =1 \text { if } E_{j^{\prime}}<\frac{E_{j-1}}{\alpha}<E_{j^{\prime}-1}
\end{aligned}
$$

and

$$
P_{j^{\prime}}=\left(E_{j^{\prime}-1}-E_{j^{\prime}}\right) / \sum_{\substack{i^{\prime} \text { in } j^{\prime} \\ m^{\prime} \text { in } k_{m}}} \phi_{i^{\prime} m^{\prime}}
$$

The term $\chi_{a j i}$, is obtained from the evaporation model constants or inelastic spectrum table $\left[\mathrm{P}_{\mathrm{n}},(\mathrm{V})_{\mathrm{n}}^{2}\right]$

$$
x_{a j i^{\prime}}=\left[P_{n_{1}(j)}-P_{n_{2}}(j)\right] / P_{n_{3}\left(i^{\prime}\right)}
$$

where $P_{n_{1}}(j)$ is the entry in the table such that

$$
\left(V^{2}\right)_{n_{1}(j)+1}>1.9132\left(E_{i},-E_{j}\right)>\left(V^{2}\right)_{n_{1}(j)}
$$

$P_{n_{2}(j)}$ is the entry in the table such that

$$
\left(V^{2}\right)_{n_{2}\left(i^{\prime}\right)+1}>1.9132\left(E_{i}-E_{j-1}\right)>\left(V^{2}\right)_{n_{2}(j)}
$$

and $P_{n_{3}\left(i^{\prime}\right)}$ is the entry such that

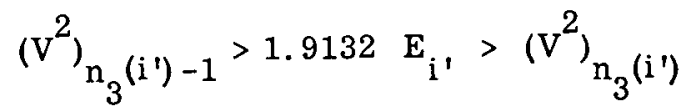


For isotopes using the evaporation model

$$
x_{a j i^{\prime}}=\frac{e^{-E_{j} / T}\left(E_{j}+T\right)-e^{-E_{j-1} / T}\left(E_{j-1}+T\right)}{T-e^{-E_{i} / T} \cdot\left(E_{i}+T\right)}
$$

where

$$
T=1.3832 \quad \sqrt{E_{i}}
$$

The fission coefficient is

$$
\mathrm{f}_{\mathrm{ajm}}=\frac{\left(E_{\left.j-1^{-E_{j}}\right)}\right.}{\sum_{\substack{i \text { in } j \\ m^{\prime} \text { in } k_{m}}} \phi_{i m^{\prime}}}\left[\sum _ { i \text { in } j } \left(\sigma_{\left.f \nu_{f}\right)_{a i}} \phi_{i m}\right.\right.
$$

while the fission spectrum coefficient. $\chi_{f}$ is obtained from the monte carlo fission spectrum data $\left(P_{n}, V_{n}\right)$

$$
\begin{aligned}
x_{f j}=\left[P_{n}(j-1)-P_{n(j)}\right. & +\frac{E_{j-1}-0.5227 v_{n(j-1)}^{2}}{0.5227\left(v_{n(j-1)+1}^{2}-v_{n(j-1)}^{2}\right)} \frac{\left(P_{n(j-1)+1}-P_{n(j-1)}\right)}{\left(E_{j-1}-E_{j}\right)} \\
& \left.-\frac{E_{j}-0.5227 v_{n(j)}^{2}}{0.5227\left(v_{n(j)+1}^{2}-v_{n(j)}^{2}\right)} \frac{\left(P_{n(j)+1}-P_{n j}\right) \mid}{E_{j-1}-E_{j+1}}\right]
\end{aligned}
$$

where $n(j)$ and $n(j-1)$ are defined by

$$
\begin{aligned}
& -0.5227 \cdot v_{n(j-1)}^{2} \leq E_{j-1}<0.5227 v_{n(j-1)+1}^{2} \\
& 0.5227 v_{n(j)}^{2} \leq E_{j} \leq 0.5227 v_{n(j)+1}^{2}
\end{aligned}
$$


While preparing the diffusion constants, an additional constant $\Sigma_{f_{j k}}$ is computed by equations identical to that for $f_{a j m}$ and $F_{j k}$ except for the replacement of $\sigma_{f} \nu_{f}$ by $\sigma_{f}$. This is used in the calculation of Burnup Constants to obtain the average value of $\nu$.

The diffusion coefficient is not computed individually for each isotope and material but is obtained for each macroscopic group and region directly from monte carlo tallies. Therefore $\mathrm{D}_{\mathrm{jk}}$ does not change as a direct result of a burnup step but is modified only after a monte carlo calculation.

$$
D_{j k}=\frac{L_{k j}}{(\hat{n} \cdot \vec{\nabla} \phi)_{k j}}
$$

For those materials and groups involving resonances, the contributions to the nuclear constants $t_{a j m}, t_{a j j}{ }^{\prime}$ ' $f_{a j m}$ are obtained by using the quantites in (109) for the values $\sigma_{\text {ait }}^{\prime}, \sigma_{\text {ais }}, \sigma_{\text {aic, }}$ and $\left(\sigma_{\mathrm{f}} \nu_{\mathrm{f}}\right)$ ai in (98), (99), and (105) in groups in which a has unresolved resonances:

$$
\begin{aligned}
& \left(\tilde{\sigma}_{f} \nu_{f}\right)_{a i}=\left(\Sigma_{f} \phi\right)_{\text {ajm }}^{\prime} \nu_{\text {far }} / \sum_{i \text { in } j} \cdot \phi_{\text {im }} N_{a m} \\
& \tilde{\sigma}_{\text {aic }}=\left(\sum_{\mathrm{c}} \phi\right)_{\text {ajm }} \\
& \tilde{\sigma}_{\text {ais }}=\left(\sum_{\text {in }} \phi\right)_{\text {ajm }} \phi_{\text {im }} N_{\text {am }} \\
& \tilde{\sigma}_{\text {ait }}=\tilde{\sigma}_{\text {fai }}+\tilde{\sigma}_{\text {aic }}+\tilde{\sigma}_{\text {ais }}
\end{aligned}
$$

Note that the quantities on the left of (109) will actually be independent of $i$ within a given macroscopic group $j$ since the monte carlo unresolved resonances tallies on the right are only made by macroscopic group. The term $\nu_{\text {far }}$ is the value given for unresolved resonance groups in a. These quantities are computed just after a monte carlo step using the $\mathrm{N}_{\text {am }}$ which were used in the monte carlo. 
The microscopic diffusion calculation constants described above are computed after each monte carlo step. Macroscopic values are obtained from Equation (95) and (97) after each burnup or monte carlo step. For example,

$$
\mathrm{T}_{\mathrm{jK}}=\sum_{\mathrm{m} \text { in } \mathrm{k}} \sum_{\mathrm{a} \text { in } \mathrm{m}} \mathrm{N}_{\mathrm{a}} \mathrm{t}_{\mathrm{ajm}} \times 10^{-24}
$$

To the $\mathrm{T}_{\mathrm{kj}}$ thus obtained must be added the quantity

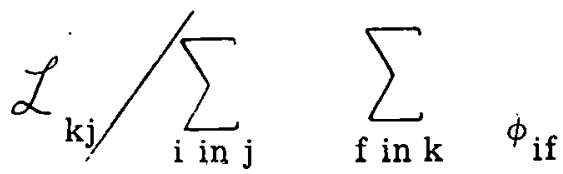

to account for the perpendicular leakage usually represented by the pseudo cross section $\mathrm{B}^{2} \mathrm{D}$. 


\section{ITERATION ON THE REACTIVITY}

The input contains a list of material indices listed in a sequential order which represent the reactivity regulation system of the reactor. Reactivity is altered by changing the concentiations in a material in this sequence. For each of the control materials, two sets of concentrations are listed in the input; the concentrations in actual use at any time are a mixture of these. When the required adjustment of reactivity is greater than that whic $h$ can be obtained by use of one of the pure concentration sets for the material, the program passes on to and begins to adjust the next region set in the sequence.

Explicitly, the control materials are those with microscopic region set numbers included in the input list:

$$
m_{1}, m_{2}, m_{3}, \ldots \ldots, m_{\alpha}
$$

Associated with each index $c_{\ell}$ in this list are two sets of concentrations: ${ }_{1} \mathrm{~N}_{\mathrm{qm} \ell}$ and ${ }_{2} \mathrm{~N}$ qm and a number $\mathrm{P}_{\ell}$ which specifies the mixture. The concentrations actually in. each control material, as identified by the concentrations stored in the normal places for the material are given by

$$
P \ell=\frac{\mathrm{N}_{\mathrm{nm \ell}}-\mathrm{N}_{\mathrm{gm} \ell}}{2_{\mathrm{qm} \ell}-\mathrm{N}_{\mathrm{qm} \ell}}
$$

A number $\ell$ indicates which of the $\mathcal{L}$ materials is currently modifiable for adjustment of criticality. On completion of a diffusion calculation, the program determines if the difference between the multiplication and unity is within specified tolerance limits, that is, if $|k-1| \leq \epsilon_{5}$. If it is, then no adjustment of control need be made, and the program preceeds to the next portion of the calculation, normally a burnup step.

If the criticality criterion is not satisfied, then a change is made in the contents of material $\mathrm{m}_{\ell}$. A set of numbers $\mathrm{C}_{\ell}$ stored in the computer provides an estimate of the dependance of $\mathrm{k}$ on $\mathrm{P}_{\ell}: \frac{\mathrm{d} k}{\mathrm{dP}}=\frac{1}{\mathrm{C}_{\ell}}$. The two sets of concentrations are chosen in such a way, that for each $\ell$, the second set of concentrations, ${ }_{2}{ }^{N}{ } m_{l}$, will lead to higher reactivity 
than the first set, ${ }_{2} \mathrm{~N}_{\mathrm{m}_{\ell}}$. Thus, $0 \leq \mathrm{P}_{\ell} \leq 1$ and $\mathrm{C}=0$. The $\mathrm{C}_{\ell}$ are initially input; following a change in $\mathrm{P}_{\ell}$ and the subsequent change in $k, C_{\ell}$ is recomputed from $\frac{\Delta \mathrm{P} \ell}{\Delta k}$. If a further change in $\mathrm{P}_{\ell}$ is required to adjust reactivity, the appropriate $\Delta \mathrm{P}_{\ell}$ is computed from this new value of $\dot{C}_{l}$, by $\Delta \mathrm{P}_{l}=\mathrm{C}_{l}(1-h)$. With the new value of $\mathrm{P}_{\ell}$, new concentrations are computed from (110). The process is continued until $|k-1| \leq \epsilon_{5}$ In the course of this series of trials, if a $P_{\ell}$ outside the range $0 \leq P_{\ell} \leq 1$, is indicated, the program will first use the extreme value of $P_{2}$ in this range, and on the next iteration will, if further adjustments are required, increase or decrease $\ell$ by one to go to the next control material. If the adjustment required is a decrease in $k$, will be decreased; if an increase in $k$ is required, $l$ will be increased. An attempt to bring $l$ outside the range $0=\ell \leq \mathcal{L}$ results in a stop signifying expiration of the reactivity life. 


\section{THE BURNUP CALCULATION}

The change in concentration of an isotope in RBU is governed by the differential equation.

$\frac{\partial N_{a m}}{\partial t}=\sum_{a^{\prime}} N_{a^{\prime} m}\left[\sum_{j} \sigma_{a^{\prime} \rightarrow a, j, k, m} \phi_{j k}+\lambda_{a^{\prime} \rightarrow a}\right]-N_{a m}\left[\sum_{j} \sigma_{a, j, k, m^{\phi}}+\lambda_{a}\right]$

$\mathrm{N}_{\mathrm{am}}$ is the atomic concentration of isotope a in material $\mathrm{m} ; \sigma_{\mathrm{a}} \rightarrow \mathrm{a}, \mathrm{j}, \mathrm{k}, \mathrm{m}$ is the microscopic cross section of isotope $a^{\prime}$ in macroscopic energy group $j$ and material $m$ for reactions producing isotope a obtained by monte carlo flux weighted averaging over the microscopic region sets containing material $m ; \phi_{j k}$ is the diffusion neutron flux density in macroscopic group $\mathrm{j}$ averaged over region $\mathrm{k} ; \lambda_{\mathrm{a}^{\prime} \rightarrow \mathrm{a}}$ is the radioactive decay constant of isotope a' for disintegrations producing isotope $a ; \sigma_{a, j, k, m}$ is the cross section of isotope $a$ in group $\mathrm{j}$ and material $\mathrm{m}$ for reactions which destroy isotope $\mathrm{a}$; and $\lambda_{a}$ is the total radioactive decay constant of isotope a. The "cross sections" in (111) contain factors to account for the energy span of group $j$ and for the reactor power level. Cross sections and fluxes in (111) are obtained from microscopic group cross sections by a procedure similar to that discussed in the previous section on Diffusion Constants. The calculation of these and the fluxes will be described in the section on Burnup Constants; in this section the discussion is limited to the solution of the sets of equations represented for each of the isotopes present in a material $\mathrm{m}$. For this purpose (111) may be rewritten

$$
\stackrel{f}{a m}_{a}=\Sigma_{a^{\prime}} \mathrm{N}_{a^{\prime} \mathrm{m}} \mathrm{G}_{\mathrm{a}^{\prime}} \text { (all } \mathrm{a}^{\prime} \text { in } \mathrm{m} \text {; all } \mathrm{m} \text { in the problem). }
$$

Equation (112) is solved to give the values of the $\mathrm{N}_{\text {am }}$ at discrete points in time, the time interval, in general, being different for each material. The time interval is expressed as the product $\mathrm{N}_{\mathrm{BU}} \Delta \mathrm{t}$ where $\Delta \mathrm{t}$ is the basic time step for the problem - the interval during which all macroscopic fluxes are assumed constant. Thus, a diffusion 
calculation is made at each interval $\Delta t$. Following a diffusion step, a burnup calculation is done for some material and, of course, it is the changes in concentrations resulting from a burnup step (or the changes in macroscopic region cross sections resulting from a monte carlo) that causes the results of two successive diffusion calculations to differ. For this reason at least one of the $\mathrm{N}_{\mathrm{BU}}$ must be unity and, no $\mathrm{N}_{\mathrm{BU}}$ can be less than unity. The solution of (112) is carried out by means of a predictor-corrector method due to Adams so that, after the first two burnup steps on a material, an estimate of the precision is available. This estimate for each isotope in a region is compared with a pair of tolerance limits, $\epsilon_{1}$ and $\epsilon_{2}$ to determine whether $\mathrm{N}_{\mathrm{BU}}$ should be increased or decreased.

\section{A. The Predictor-Corrector Method}

Calculations of the concentrations are made using estimates of the first through fourth derivatives obtained from the differences of values of the previous first derivatives. Thus predictions are made with

$$
\begin{aligned}
& \mathrm{p}^{\rho}=\mathrm{N}^{\rho-1}+\mathrm{N}_{\mathrm{BU}} \Delta \mathrm{t} \sum_{\mathrm{i}=0}^{3} \alpha_{i}\left(\nabla^{\mathrm{i}} \stackrel{\mathrm{N}}{\rho-1}\right. \\
& \alpha_{0}=1, \alpha_{1}=\frac{1}{2}, \alpha_{2}=\frac{5}{12}, \alpha_{3}=\frac{3}{8}
\end{aligned}
$$

using the backward differences

$$
\begin{aligned}
& \stackrel{\circ}{\nabla} \rho \equiv y^{\rho} \\
& \nabla_{\mathrm{y}} \rho \equiv \mathrm{y}^{\rho}-\mathrm{y}^{\rho-1} \\
& \nabla^{2} y^{\rho} \equiv \frac{1}{\nabla y} \rho-\frac{1}{\nabla y}{ }^{\rho-1}=y^{\rho}-2 y^{\rho-1}+y^{\rho-2} \\
& \frac{\mathrm{i}}{\nabla \mathrm{y}} \rho \equiv \stackrel{\mathrm{i}}{\nabla}-1^{\mathrm{y}} \rho-\stackrel{\mathrm{i}}{\nabla}-1_{\mathrm{y}} \rho-1
\end{aligned}
$$

In $(113)^{*}$ the superscript $\rho$ indicates the time at which the concentrations and their rates of change apply. The term ${ }_{p} N^{\rho}$ is the concentration at time $t_{\rho}$ estimated from data available

* Equation (113) as well as Equation (115) and (118) are derived in Section X.III D. 
at time $\mathrm{t}_{\rho}-\mathrm{N}_{\mathrm{BU}} \Delta \mathrm{t} \equiv \mathrm{t} \rho-1$. The data available at this time is the concentration, $\mathrm{N}^{\rho-1}$, the rate of change at $\mathrm{t}_{\rho-1}, \stackrel{\circ}{\mathrm{N}}^{\rho-1}$; and the higher order differences of $\stackrel{\circ}{\mathrm{N}},\left(\frac{\dot{\mathrm{i}}}{\nabla} \stackrel{\mathrm{N}}{\mathrm{N}}\right) \rho-1$ $(i=1,2,3)$ which y.ield information on the second through fourth derivatives of $\mathrm{N}$ in recent time steps. The zeroth order difference, $(\stackrel{\circ}{\nabla} \mathrm{N}){ }^{\rho-1}=\stackrel{\circ}{N}^{\rho-1}$ is obtained from (112) using the fluxes and (corrected) concentrations at $t_{\rho-1}$. The fluxes used for this purpose depend, of course, on the properties of all regions in addition to those containing material $\mathrm{m}$; the implications of this will become more apparent in the subsequent discussion.

When the rate of change of concentration of t $\rho$ is available, another estimate of $\mathrm{N}^{\rho}$ may be made; this is termed the corrector:

$$
\begin{aligned}
& \mathrm{c}^{\rho}=\mathrm{N}^{\rho-1}+\mathrm{N}_{\mathrm{BU}} \Delta \mathrm{t} \sum_{\mathrm{i}=0}^{j} \beta_{\mathrm{i}}\left(\nabla^{\mathrm{i}} \stackrel{\mathrm{N}}{\rho}^{\rho}\right) \\
& \beta_{0}=1, \beta_{1}=-\frac{1}{2}, \beta_{2}=-\frac{1}{12}, \beta_{3}=-\frac{1}{24}
\end{aligned}
$$

The agreement between ${ }_{\mathrm{p}} \mathrm{N}^{\rho}$ and ${ }_{\mathrm{c}} \mathrm{N}^{\rho}$ is a measure of the extent to which the time interval $\mathrm{N}_{\mathrm{BU}} \Delta \mathrm{t}$ is satisfactory:- If the two disagree excessively this is an indication either that changes in concentration of other isotopes in the same material or higher terms in the expansion of the rate of depletion of the isotope in question lead to a requirement for a shorter time step or that indirect effects of changes in other materials are causing errors. These indirect effects may be understood as follows.

Consider two materials $\mathrm{A}$ and $\mathrm{B}$ and suppose that at the time $t$ the changes in concentration of one of the isotopes in material $B$ begin to produce rapid changes in the fluxes in regions containing materials $\mathrm{A}$ and $\mathrm{B}$. If the predictor in material $\mathrm{A}$ is computed from fluxes obtained before this rapid change and the corrector is computed from fluxes obtained after the calculation of new concentrations in material $B$, an excessive disagreement between the predicted and corrected concentrations of isotopes in material A may be expected. 
This will cause the program to decrease $\mathrm{N}_{\mathrm{BU}} \cdot$. If $\mathrm{N}_{\mathrm{BU}_{\mathrm{A}}}>\mathrm{N}_{\mathrm{BU}}$ this course of action will lead to an improvement in the accuracy of calculation in material A because the changes in fluxes will be more frequently taken into account. If $\mathrm{N}_{\mathrm{BU}_{\mathrm{A}}}{ }^{\prime} \leq \mathrm{N}_{\mathrm{BU}_{\mathrm{B}}}$, decreasing $\mathrm{N}_{\mathrm{BU}}$ cannot be expected to improve the accuracy in material $A$. The same changes which caused disagreement in $A$, will presumably cause disagreement in $B$, however, so that $\mathrm{N}_{\mathrm{BU}_{\mathrm{B}}}$ will be decreased leading to higher accuracy in both materials. After the effects of the decrease in $\mathrm{N}_{\mathrm{BU}_{\mathrm{B}}}$ have been felt, the program may find it possible to restore $\mathrm{N}_{\mathrm{BU}}$ to its former value.

\section{B. Changing the Time Interval}

The changes in the $\mathrm{N}_{\mathrm{BU}}$ are controlled through the comparison of relative errors in predicted and corrected concentrations with two tolerance limits, $\epsilon_{1}$ and $\epsilon_{2}$. If

$$
\left|\frac{p^{\mathrm{N}}-c^{\mathrm{N}} \mathrm{a}}{\mathrm{c}^{\mathrm{N}} \mathrm{a}}\right|<\epsilon_{2}
$$

for all isotopes in material $\mathrm{m}$, then $\mathrm{N}_{\mathrm{BU}}$ will be increased by a factor of two for the next prediction. If

$$
\left|\frac{p^{N}{ }^{-} c^{N} a}{c^{N}}\right|>\epsilon_{1}
$$

for any isotope in material $\mathrm{m}$ then $\mathrm{N}_{\mathrm{BU}}$ will be decreased by a factor of 2 for the next prediction.

When an $\mathrm{N}_{\mathrm{BU}}$ is decreased, backward differences, $\stackrel{i}{\nabla}$, corresponding to the new time interval are required for the next prediction. The philosophy followed here is that $\epsilon_{1}$ is sufficiently small that when a relative error greater than $\epsilon_{1}$ is detected at time $t$, the data available will still be satisfactory for prediction to the time point at $t+\mathrm{N}_{\mathrm{BU}} \Delta \mathrm{m}$. (In this expression $\mathrm{N}_{\mathrm{BU}}$ is the new value.) With this point of view, the rates of change 
of concentration at recent intermediate points may be found using Newton's interpolation formulas. If $f(t)$ is the latest value just computed, $N_{B U} \Delta t$ the new time interval and subscripts on the $\nabla^{i}$ indicate whether they are associated with the old or new interval

$$
\begin{aligned}
& \stackrel{\circ}{N}\left(t-2 N_{B U^{\Delta}} \Delta t\right)=\stackrel{\circ}{N}(t)-\nabla_{\text {old }}^{1} \stackrel{\circ}{N}(t) \\
& \stackrel{\circ}{N}\left(t-N_{B U} \Delta t\right)=\sum_{i=0}^{3} \gamma_{i}\left|\nabla_{\text {old }}^{i} \stackrel{\circ}{N}(t)\right| \\
& \stackrel{\circ}{N}\left(t-3 N_{B U^{\Delta t}}{ }^{3}\right)=\sum_{i=0}^{3} \gamma_{i}^{\prime} \mid \nabla_{\text {old }}^{i} \stackrel{\circ}{N}(t) i \\
& \gamma_{0}=1, \gamma_{1}=-\frac{1}{2}, \gamma_{2}=-\frac{1}{8}, \gamma_{3}=-\frac{1}{16} \\
& \gamma_{0}^{\prime}=1, \gamma_{1}^{\prime}=-\frac{3}{2}, \quad \gamma_{2}^{\prime}=\frac{3}{8}, \quad \gamma_{3}^{\prime}=\frac{1}{16} \\
& \nabla^{\circ} \stackrel{0}{N}(t)=\stackrel{\circ}{N}(t) \\
& \nabla_{\text {new }}^{1} \stackrel{\circ}{N}(t)=\stackrel{\circ}{N}(t)-\stackrel{\circ}{N}\left(t-N_{B U} \Delta t\right) \\
& \nabla_{\text {new }}^{2} \stackrel{\circ}{N}(t)=\stackrel{\circ}{N}(t)-2 \stackrel{\circ}{N}\left(t-N_{B U} \Delta t\right)+\stackrel{\circ}{N}\left(t-2 N_{B U} \Delta t\right) \\
& \nabla_{\text {new }}^{3} \stackrel{\circ}{N}(t)=\stackrel{\circ}{N}(t)-3 \stackrel{\circ}{N}\left(t-N_{B U} \Delta t\right)+3 \stackrel{\circ}{N}\left(t-2 N_{B U} \Delta t\right)-\stackrel{\circ}{N}\left(t-3 N_{B U} \Delta t\right)
\end{aligned}
$$

When an $\mathrm{N}_{\mathrm{BU}}$ is to be increased the $\nabla_{\text {new }}^{\mathrm{i}}$ are computed for the larger time interval. If the terms ${ }_{p} N(t)$ and ${ }_{c} N(t)$ are the values of concentration which agreed sufficiently well to indicate the possibility of increasing the time interval to the new value $N_{B U} \Delta t$, then the quantities available are

$$
\begin{aligned}
& \left.\stackrel{\circ}{\mathrm{N}}(\mathrm{t}) \equiv \dot{\nabla}_{\text {old }}^{\circ} \stackrel{\circ}{\mathrm{N}}(\mathrm{t}) ; \stackrel{\circ}{\mathrm{N}} \mid \mathrm{t}-\frac{1}{2} \mathrm{~N}_{\mathrm{BU}} \Delta \mathrm{t}\right) \equiv \nabla_{\text {old }}^{\mathrm{O}} \stackrel{\circ}{\mathrm{N}}\left(\mathrm{t}-\frac{1}{2} \mathrm{~N}_{\mathrm{BU}} \Delta \mathrm{t}\right) ; \\
& \left.\nabla_{\text {old }}^{1} \mid \mathrm{t}-\frac{1}{2} \mathrm{~N}_{\mathrm{BU}} \Delta \mathrm{t}\right) ; \nabla_{\text {old }}^{2} \stackrel{\circ}{\mathrm{N}}\left(\mathrm{t}-\frac{1}{2} \mathrm{~N}_{\mathrm{BU}} \Delta \mathrm{t}\right) ; \nabla_{\text {old }}^{3} \stackrel{\circ}{\mathrm{N}}\left(\mathrm{t}-\frac{1}{2} \mathrm{~N}_{\mathrm{BU}} \Delta \mathrm{t}\right)
\end{aligned}
$$


From these $\stackrel{\circ}{N}\left(t-N_{B U} \Delta t\right)$ and $\stackrel{\circ}{N}\left(t-2 N_{B U} \Delta t\right)^{\prime}$ may be found leading to values $\nabla_{\text {new }}^{0} \stackrel{\circ}{N}(t)$, $\nabla_{\text {new }}^{1} \stackrel{\circ}{N}(t)$, and $\nabla_{\text {new }}^{2} \stackrel{\circ}{N}(t)$ by equations similar to (121).

, There are a number of complications modifying the procedures described so far in this section. These arise primarily from the fact that the set. of $\nabla^{i}$ is not always complete. The modifications need not be discussed in detail here, essentially the program uses what information is available, terminating the series in (114), (115), and (118) before the $\nabla^{3}$ term if fewer $\nabla^{i}$ are available.

A second modification arises from the necessity that no $\mathrm{N}_{\mathrm{BU}}$ be less than unity and that at least one $\mathrm{N}_{\mathrm{BU}}$ be equal to unity. After each burnup in which the changes in the $\mathrm{N}_{\mathrm{BU}}$ described above have been made for each of the materials for which the relative difference between predicted and corrected values dictates a change, all $\mathrm{N}_{\mathrm{BU}}$ are examined to determine if these conditions hold. If any $\mathrm{N}_{\mathrm{BU}}$ is one half, the basic time stép, $\Delta t$, is decreased by a factor of two and all $\mathrm{N}_{\mathrm{BU}}$ multiplied by two. Since this does not affect any values of $N_{B U_{m}} \Delta t$, it is not necessary to change any of the $\nabla_{\text {am }}^{i}$. Similarly if all $\mathrm{N}_{\mathrm{BU}}$ are greater than one, $\Delta t$ is doubled and the $\mathrm{N}_{\mathrm{BU}}$ are halved. Because of the fact that increasing a particular $\mathrm{N}_{\mathrm{BU}}$ may result in a change in the basic time step, $\Delta t$, affecting the points in time at which it is possible to do burnup calculations on other materials, an increase in an $\mathrm{N}_{\mathrm{BU}}$ can only be made at certain times. Synchronization of all materials is assured by requiring that the time at which the change in $\mathrm{N}_{\mathrm{BU}}$ is to be made is an integral multiple of the new time step $2 \mathrm{~N}_{\mathrm{BU}_{\mathrm{m}}} \Delta \mathrm{t}$. 


\section{BURNUP CONSTANTS}

The evaluation of constants for the burnup calculation proceeds in two steps, as does that of the diffusion constants. Following a monte carlo calculation, is a computation of the effective cross sections for macroscopic groups per unit concentration in each material and per unit flux density in diffusion region $\mathrm{k}$ containing that material

$$
\begin{aligned}
& \sigma_{a \rightarrow a, j, k, m}=2.72 \times 10^{-6}\left(E_{j-1}-E_{j}\right) p\left[\sum_{i} \sigma_{j} \sigma_{a \rightarrow a, j}^{\prime} \phi_{i m k}\right] / \sum_{m^{\prime} \text { in } k_{m}} \phi_{i^{\prime}} \\
& \phi_{\text {im }}=\sum_{\text {f, containing } \mathrm{m}} \phi_{\text {if }}
\end{aligned}
$$

Equation (121) contains several factors included for convenience which makes the quantity on the left not strictly a cross section. $P$ is the input value of the operating power density in kilowatts per cubic centimeter. It is actually obtained by dividing the total thermal power output of the reactor by the volume represented in the diffusion calculation. The volume is given by $\frac{1}{\rho+1} \mathrm{R}_{\mathrm{N}}{ }^{\rho+1}$. The constant $2.72 \times 10^{-6}$ includes a factor of $10^{-24}$ to convert barns to square centimeters, a factor to convert power density to density of fissions per second, and a factor to convert time from days to seconds. ${ }^{*}$ To obtain this constant the energy released per fission was assumed to be $198 \mathrm{mev}$.

To obtain the rate of production of isotope a in atoms per $\mathrm{cm}^{3}$ per day per unit concentration of isotope $a^{\prime}$ (in material $\mathrm{m}$ ) by reactions represented by $\sigma_{\mathrm{a}^{\prime} \rightarrow \mathrm{a}^{\prime}}$ it is necessary to multiply by the diffusion fluxes integrated over region $\mathrm{k}$.

$$
\dot{\mathrm{N}}_{\mathrm{am}}=\mathrm{N}_{\mathrm{a}^{\prime} \mathrm{m}} \sum_{\mathrm{j}} \sigma_{\mathrm{a}^{\prime} \rightarrow \mathrm{a}, \mathrm{jkm}} \phi_{\mathrm{jk}}+\text { terms due to other reactions }
$$

where

$$
\begin{aligned}
& \phi_{j \mathrm{k}}^{\prime}=\frac{\Delta \mathrm{r}_{\mathrm{k}}}{\mathrm{v}_{\mathrm{k}}}\left[\frac{1}{2} \mathrm{r}_{\mathrm{N}_{\mathrm{k}-1}}^{\rho} \phi_{\mathrm{N}_{\mathrm{k}-1}}+\sum_{\mathrm{n}=\mathrm{N}_{\mathrm{k}-1}+1}^{\mathrm{N}_{\mathrm{k}-1}} \mathrm{r}_{\mathrm{n}}^{\rho} \phi_{\mathrm{jn}}+\frac{1}{2} \mathrm{r}_{\mathrm{N}_{\mathrm{k}}}^{\rho} \phi_{\mathrm{jN}}\right] \\
& \phi_{\mathrm{jk}}=\nu \phi_{\mathrm{jk}}^{\prime}
\end{aligned}
$$

* Although the unit used for time in the monte carlo portion of RBU is microseconds, the burnup portion uses days. 


$$
\mathrm{v}_{\mathrm{k}}=\Delta \mathrm{r}_{\mathrm{k}}\left[\frac{1}{2} \mathrm{r}_{\mathrm{N}_{\mathrm{k}-1}}^{\rho}+\sum_{\mathrm{n}=\mathrm{N}_{\mathrm{k}-1}+1}^{\mathrm{N}_{\mathrm{k}}-\mathrm{i}} \mathrm{r}_{\mathrm{n}}^{\rho}+\frac{1}{2} \mathrm{r}_{\mathrm{N}}^{\rho}\right]
$$

The factor $\nu$ in (122) is the average number of neutrons per fission arising from the fact that the $\Theta_{n j}$ are normalized to one source neutron per unit volume.

$$
\nu=\frac{\sum_{k} \sum_{j} F_{k j} \phi_{k j}}{\sum_{k} \sum_{j} \Sigma_{f_{k j}} \phi_{k j}^{\prime}}
$$

The appropriate cross sections to be used in equation (121) are determined from the table of direct descendants and modes of production prepared by the cross section preparation routine from the basic library. Cross sections which may be included in (121) are $\sigma_{c}, \sigma_{n, 2 n}$, and $\sigma_{f}$.

As indicated in equation (111), another term in (122) involves the total destruction cross section for isotope a. This is $\sigma_{c}+\sigma_{n, 2 n}+\sigma_{f}$. Finally (111) involves the radioactive decay terms $\lambda_{a^{\prime} \rightarrow a}$ and $\lambda_{a}$ which are used exactly as they appear in the list of properties of the isotopes and are in reciprocal days. The appropriate identification between $\lambda_{a^{\prime} \rightarrow a}$ and $a$ is made through the table of direct descendants and modes.

For isotopes having random resonances in a group $i$ within group $j$, the $\sigma_{a^{\prime} \rightarrow a, i}$ in (121) are not available from basic cross section data. They are instead obtained as in equation (109) directly. from the monte carlo tallies. 


\section{CROSS SECTION PREPARATION ROUTINE*}

The purpose of the cross section preparation routine, a part of the input program, is to assemble data on isotope properties from a basic library of isotopic data and a relatively small amount of problem input. The input program selects the necessary data from the library, organizes it into cross sections corresponding to the energy group structure specified in the program input, and writes the information on a magnetic tape to be used by the main RBU program. In addition to the basic library and problem input, a tape, containing a cumulation of all previous output of the cross section preparation routine, which pertained to the same energy group structure as the present problem, is used by the input program. The input program thus makes use of any of the necessary information that happens to be already available on the cumulative tape and, in addition to the tapes required for a particular problem, produces a new cumulative tape. The essential data, aside from the basic library to be supplied to the cross section preparation routine is: the energy limits of the microscopic groups, list of isotopes initally present in the reactor, and the ambient temperatures in the regions in which these isotopes are. Using this information and the basic library, the routine determines any additional isotopes which will appear in the reactor during operation as a result of burnup and it computes the average cross sections for each microscopic energy group for each of the isotopes to be required. The temperature is used in the calculation of Doppler broadened resonances.

In addition to this essential information, some additional data may be supplied for the treatment, in the monte carlo part of the calculation, of low energy scattering. For those isotopes requiring it, special atomic weights for use in the center of mass-laboratory system conversion on near thermal collisions may be given and probability distributions of velocity increments to be added to the emergent velocities in such collisions may be supplied. The special atomic weights consist of a set of $\mathrm{G}_{\mathrm{q}}-1$ numbers to be used in groups

* The input program including the cross section preparation routine is presently being coded by members of the staff of the Hanford Laboratories Operation. It will be described in detail in a report shortly to be issued by them. 
$I-G_{. q}+2$ through I for the $q^{\text {th }}$ isotope in the input. The probability distributions are given in the form of a table of probabilities and velocity increments for each of several

distributions; the distribution to be used for a particular isotope is identified by specifying with the isotope's input data an index referring to the appropriate table. An index of zero associated with an isotope signifies that velocity increments need not be used for it.

The basic library includes a table giving all the descendants, direct and indirect of each isotope in the library, and a table of the direct descendants only of each isotope with an index indicating whẹther the transformation is the result of neutron capture $(n, 2 n)$, fission, or radioactive decay reactions. The index indicating fission may have one of six values to indicate which of the six fission yields given with the parent isotope data applies to this particular descendant. In addition, the general nuclear data includes tables of spectra for inelastic collisions. An index may be given for each isotope in the isotope data selecting one of these tables to be associated with it. Also included in the general nuclear data are sets of resonance structure parameters. The $\rho^{\text {th }}$ set includes values of $\Gamma_{\mathrm{n}}, \Gamma_{\mathrm{f}}, \Gamma_{\gamma}, \mathrm{g}$ and $\mathrm{E}_{\mathrm{o}}$ for each of $\mathrm{N}_{\mathrm{E}_{\mathrm{o}_{\rho}}}$ resolved resonances, a value of $\nu_{\mathrm{fr}}$ and $\sigma_{\mathrm{p}}$ for all resonances for isotopes to which the set applies and a value of $\bar{\Gamma}_{\mathrm{n}}{ }^{\prime} \Gamma_{\gamma}$, $\widetilde{\Gamma}_{\mathrm{f}}, \mathrm{g}$ and $\mathrm{D}$ for the unresolved resonances. In addition, values of $I_{1 \rho}, I_{2}$ and $I_{3 \rho}$ define the resolved and unresolved ranges. An index $\rho$ associated with each isotope indicates which set is to be used for it.

The data associated with a particular isotope in the basic library consists of a general section and a section giving the energy dependent properties. The general data includes the isotope's name or index, its atomic weight, six fission yields, the mean lifetime for radioactive decay, the energy below which elastic scattering is isotropic, the potential scattering cross section for use in resonances, either an index selecting one of the inelastic spectrum tables or a level spacing for use in the evaporation model inelastic spectrum, and an index selecting one of the sets of resonance parameters.

The energy dependent data is given for a set of ranges the widths of which vary from range to range and from isotope to isotope, being chosen as is approriate to the range and isotope in question. 
With each range (for each isotope) is associated an index, $\delta$, identifying the manner in which the properties vary within that range. The possible types of variation are described in Table I

TABLE I

\section{VARIATION OF CROSS SECTIONS WITHIN THE ENERGY RANGE $\mathrm{E}_{\mathrm{n}} \leq \mathrm{E} \leq \mathrm{E}_{\mathrm{n}-1}$}

\begin{tabular}{|c|c|c|c|c|c|}
\hline Quantity & $\delta=1$ & $\delta=2$ & $\delta=3$ & $\delta=4$ & $\delta=5$ \\
\hline$\sigma_{\mathrm{e} 1}$ & Linear & Constant & Lineal & Breit Wigner & $\begin{array}{l}\text { No group constants are } \\
\text { computed when } \delta=5 . \delta=5\end{array}$ \\
\hline$\sigma_{\mathbf{c}}$ & Linear & Linear & $\frac{1}{\mathrm{~V}}$ & Breit Wigner & $\begin{array}{l}\text { is used when the cross } \\
\text { sections in the monte carlo }\end{array}$ \\
\hline$\sigma_{f}$ & Linear & Linear & $\frac{1}{\mathrm{~V}}$ & Breit Wigner & $\begin{array}{l}\text { are to be computed from the } \\
\text { resonance tables alone and the }\end{array}$ \\
\hline$\sigma_{\text {inel }}$ & Linear & Linear & $\begin{array}{l}\text { Assumed not } \\
\text { present }\end{array}$ & $\begin{array}{l}\text { Assumed not } \\
\text { present }\end{array}$ & $\begin{array}{l}\text { diffusion and burnup cross } \\
\text { sections from the monte }\end{array}$ \\
\hline$\nu_{\mathrm{f}}$ & Constânt & Constant & Constant & Constant & carlo resonance tallies. ${ }^{*}$ \\
\hline$\nu_{\text {inel }}$ & Constant & Constant & $\begin{array}{l}\text { Assumed not } \\
\text { present }\end{array}$ & $\begin{array}{l}\text { Assumed not } \\
\text { present }\end{array}$ & \\
\hline$a_{0}$ & Constant & Constant & Constant & Constant & \\
\hline$a_{1}$ & Constant & Constant & Constant & Constant & . \\
\hline$a_{2}$ & Constant & Constant & Constant & Constant & \\
\hline
\end{tabular}

* $\delta=1,2$ or 3 may also be used in the resonance range in which case the main program will use the sum of the group values and the values computed from the resonance tables.

In Table I the term linear means that the cross section is assumed to have the form

$$
\begin{aligned}
& \sigma(E)=\sigma_{n}+\frac{\sigma_{n-1}-\sigma_{n}}{E_{n-1}-E_{n}}\left(E-E_{n}\right) \\
& E_{n} \leq E \leq E_{n-1}
\end{aligned}
$$

The term $\frac{1}{V}$ means that

$$
\begin{aligned}
& \sigma(\mathrm{E})=\frac{\sigma_{\mathrm{n}}}{\mathrm{E}^{1 / 2}} \\
& \mathrm{E}_{\mathrm{n}} \leq \mathrm{E} \leq \mathrm{E}_{\mathrm{n}-1}
\end{aligned}
$$


The term Brit Wigner if $\delta=4$ means that

$$
\begin{aligned}
& \sigma_{\mathrm{s}}(\mathrm{E})=\sigma_{\mathrm{s}_{\mathrm{o}}} \psi(\mathrm{X}, \theta)+2\left(\frac{\mathrm{Q}+1}{\mathrm{a}}\right) \sqrt{\sigma_{\mathrm{p} \mathrm{s}_{\mathrm{o}} \mathrm{g}}} \times(\mathrm{X}, \theta)+\sigma_{\mathrm{p}}, \\
& \sigma_{\mathrm{c}}(\mathrm{E})=\frac{\sigma_{\mathrm{c}_{\mathrm{o}}}}{\sqrt{\mathrm{E}}} \psi(\mathrm{X}, \theta), \\
& \sigma_{\mathrm{f}}(\mathrm{E})=\frac{\sigma_{\mathrm{o}}}{\sqrt{\mathrm{E}}} \psi(\mathrm{X}, \theta),
\end{aligned}
$$

where

$$
\begin{aligned}
& x=\frac{2\left[\left(\frac{a}{a+1}\right) E-E_{0}\right]}{\Gamma} \\
& \Theta=3.446 \times 10^{-4} \frac{\mathrm{ET}}{\mathrm{A \Gamma}_{\Gamma}^{2}}\left(\frac{a}{a+1}\right)^{2} \\
& \psi(\mathrm{X}, \Theta)=\frac{1}{2 \sqrt{\pi \Theta}} \cdot \int_{-\infty}^{\infty} \frac{\mathrm{e}^{-(\mathrm{X}-\mathrm{Y})^{2} / 4 \theta}}{1+\mathrm{Y}^{2}} \mathrm{dy} \\
& \chi(X, \Theta)=\frac{1}{2 \sqrt{\pi \Theta}} \int_{-\infty}^{\infty} \frac{\mathrm{Ye}^{-(\mathrm{X}-\mathrm{Y})^{2} / 4 \theta}}{1+\mathrm{Y}^{2}} \mathrm{dY} \text {, } \\
& \sigma_{\mathrm{s}_{\mathrm{o}}}=\frac{2.607 \times 10^{6}}{\left(\Gamma_{\mathrm{n}}+\Gamma_{\gamma}^{\prime}+\Gamma_{\mathrm{f}}\right)^{2}} \frac{\Gamma_{\mathrm{n}}^{2}}{\mathrm{E}_{\mathrm{o}}} \mathrm{g}\left(\frac{1+a}{a}\right) \\
& \sigma_{c_{0}^{-}}=\frac{2.607 \times 10^{6}}{\left(\Gamma_{n}+\Gamma_{\gamma}+\Gamma_{f}\right)^{2}} \frac{\Gamma_{n}}{\sqrt{E_{o}}} \Gamma_{\gamma} g\left(\frac{1+a}{a}\right)^{3 / 2}, \\
& \sigma_{\mathrm{f}}=\frac{\Gamma_{\mathrm{f}}}{\Gamma_{\gamma}} \sigma_{\mathrm{c}} .
\end{aligned}
$$

$-67-$ 
Equations (127) and (128) are derived in section XII C. The evaluation of $\psi$ and $\chi$ is described in section XIII $B$. In the integration of Breit Wigner cross sections over a range $\mathrm{E}_{\mathrm{n}} \leq \mathrm{E} \leq \mathrm{E}_{\mathrm{n}-1}$, for $\delta=4$ the functions are evaluated at ten points within the range and integrated by Simpson's Rule.

When a resonance parameter table is specified, the cross sections are evaluated in the monte carlo portion of the main program as described in sections $V, E$ and $F$. This is equivalent to (128), except for the addition of factors to account for the higher angular momentum states and for the use of unresolved as well as resolved resonances. The significance of a range with $\delta=5$ is simply that no cross section values need be specified for that range because the entire cross section may be computed from the resonance parameter table. On the other hand, it is possible to have a range with $\delta=1,2$, or 3 within an isotope's resonance range. In such a case, the cross sections used by the new program will be the sum of those computed from the resonance tables and the group values. 


\section{MATHEMATICAL DETAILS}

\section{A. Selection from Probability Distributions}

\section{Random Numbers}

The process basic to all random selection techniques in $\mathrm{RBU}$ is the choosing of a number from a universe of numbers uniformly distributed between zero and one. Because of the importance of numbers selected from this distribution, they are generally called simply random numbers in discussions of monte carlo calculations. As in virtually all monte carlo calculations, the method is not actually random but involves the use of a recipe which leads to numbers which collectively have the properties of a set chosen by a random process; these are generally called pseudo-random numbers. The properties of importance here are that the mean of any reasonably large sample approaches the mid point of the sample range as the sample size increases, that they are distributed over the range with adequate fineness, that the frequencies of lengths of increasing and decreasing chains be distributed in the same way as those of actually random numbers and that no obvious regularities occur. It is usual to require also that the cycle length of the process be relatively long and it is, of course, also important that the process involve only a very small amount of computing time. In general, because of the complexity of monte carlo calculations, it is desirable to apply more rigid requirements than appear superficially to be necessary in selecting a random number generator.

A method which has been shown theoretically to produce numbers having the properties of those obtained from a random selection is the multiplicative method. In this technique

$$
\mathrm{R}_{\mathrm{n}+1}=\left(\mathrm{k} \cdot \mathrm{R}_{\mathrm{n}}\right) \bmod \rho
$$

where $\mathrm{k}$ and $\mathrm{R}_{\mathrm{O}}$ have certain properties and $\rho$ is the word length of the computer. In RBU

$$
\begin{aligned}
& \mathrm{k}=5^{15} \\
& \mathrm{R}_{\mathrm{o}}=(247162405723)_{8} \\
& \rho=2^{35}
\end{aligned}
$$




\section{Selection of Distance to Collision}

The selection of the number of mean free paths a particle will travel before a collision is made from the distribution

$$
\frac{\mathrm{dP}(\mathrm{S} / \lambda)}{\mathrm{d}(\mathrm{S} / \lambda)}=-\mathrm{e}^{-\mathrm{S} / \lambda}
$$

The method is based on the fact that equal increments in the logarithm of the cumulative probability represent equal intervals in the argument. It consists of selecting an interval in the logarithm of $P$ by repetitive comparison of a random number with 0.5 . For each successive random number less than 0.5 , the natural logarithm of two is added to the upper and lower limits of the range from which $S / \lambda$ is to be selected. The sequence of comparisons is stopped on the first random number greater than 0.5 thus determining an interval within which $S / \lambda$ is to be selected. A final random number determines, by a table lookup, which of eight possible values within the interval is used.

\section{Random Cosines}

The direction cosines are determined by a method which avoids the necessity of evaluating trigonometric functions just as the previous technique avoided logarithms. To do this $\alpha, \beta$, and $\gamma$ are defined in terms of spherical coordinates

$$
\begin{aligned}
& \alpha=\cos \phi \sin \theta \\
& \beta=\sin \phi \sin \theta \\
& \gamma=\cos \theta
\end{aligned}
$$

The value of $\gamma$ is then selected as a random number between -1 and +1 by

$$
\gamma=2 \xi_{1}-1
$$

where $\xi_{1}$ is uniformly distributed between 0 and 1 . Two more random numbers $\xi_{2}$ and $\xi_{3}$ are selected. These are accepted only if the sum of their squares is less than or equal to unity; otherwise two more are chosen until this condition is fulfilled. The ratio

$$
\frac{\xi_{2}}{\xi_{3}}
$$

is then the tangent of an angle, $\phi$, randomly chosen from a universe uniformly distributed between 0 and $\frac{\pi}{2}$. Finally, 


$$
\begin{aligned}
& \alpha= \pm \xi \sqrt[3]{\frac{1-\dot{\gamma}^{2}}{\xi_{2}^{2}+\xi_{3}^{2}}} \\
& \beta= \pm \xi_{2} \sqrt{\frac{1-\gamma^{2}}{\xi_{2}^{2}+\xi_{3}^{2}}}
\end{aligned}
$$

where the two signs are determined by selection of two random bits.

Although this method appears complicated, it is, due to its efficiency, faster than the obvious method of choosing and normalizing three random numbers the sum of whose squares is less than or equal to unity.

\section{Selection of Resonance Partial Widths}

The fission width is distributed as

$$
d P(v)=e^{-v} d v
$$

where

$$
v=\frac{\Gamma_{\mathbf{f}}}{\Gamma_{\mathrm{f}}}
$$

Selection of $\mathrm{v}$, then, is by the same method as that of the number of mean free paths to collision described above.

The reduced neutron width is distributed as

$$
d P(u)=\sqrt{\frac{2}{\pi}} e^{-u^{2} / 2} d u
$$

where

$$
u=\left(\frac{\Gamma_{\text {no }}}{\bar{\Gamma}_{\text {no }}}\right)^{1 / 2}
$$

The method used in RBU is to select two random numbers $L_{1}$ and $L_{2}$ from an exponential distribution as in the selection of a distance to collision above. If $\left(L_{1}-1\right)^{2} \leq 2 L_{2}$, $u$ is taken to be equal to $L_{1}$. Otherwise two more random logarithms are selected and the process repeated until a pair satisfying the condition is found. 
The probability than an $\mathrm{L}_{1}$ will be obtained and accepted is

$$
\begin{aligned}
P_{\operatorname{acc}}\left(L_{1}\right) & =\int_{\left(L_{1}-1\right)^{2} / 2}^{\infty} P\left(L_{1}\right) P\left(L_{2}\right) d L_{2} \\
& =e^{-L_{1} \int_{\left(L_{1}-1\right) / 2}^{\infty} e^{-L_{2}} \mathrm{dL}_{2}}
\end{aligned}
$$

which corresponds to (136).

\section{Selection from the Evaporation Model Inelastic Spectrum}

The probability distribution is

$$
\mathrm{dP}(\mathrm{E})=\mathrm{CE} \mathrm{e}^{-\mathrm{E} / \mathrm{T}} \mathrm{dE}
$$

The sum of two random logarithms has the distribution

$$
P(X)=\int_{0}^{X} e^{-Y} e^{-(X-Y)} d Y=X e^{-X}
$$

Thus the sum of two numbers, $\mathrm{X}$ and $\mathrm{Y}$, each distributed as $\mathrm{e}^{-\mathrm{E} / \mathrm{T}}$ has the distribution required.

B. The Calculation of PSI and CHI

\section{General}

Psi and Chi are defined as follows:

$$
\begin{aligned}
& \psi(\mathrm{X}, \theta)=\frac{1}{\sqrt{4 \pi \theta}} \cdot \int_{-\infty}^{\infty} \frac{\exp \left[-(\mathrm{Y}-\mathrm{X})^{2} / 4 \theta\right]}{1+\mathrm{Y}^{2}} d \mathrm{Y} \\
& \chi(\mathrm{X}, \theta)=\frac{1}{\sqrt{4 \pi \theta}} \int_{-\infty}^{\infty} \frac{\mathrm{Y}\left[\exp -(\mathrm{Y}-\mathrm{X})^{2} / 4 \theta\right]}{1+\mathrm{Y}^{2}} d y
\end{aligned}
$$

It is clear from the value of the integral of the exponential alone that

$$
0<\psi(\mathrm{X}, \theta) \leq 1 ;-1<\chi(\mathrm{X}, \theta)<1 .
$$

Further

$$
\psi(-\mathrm{X}, \theta)=\psi(\mathrm{X}, \theta) ; \chi(-\mathrm{X}, \theta)=-\chi(\mathrm{X}, \theta)
$$


For each of the two functions, two series are derived, one uniformly convergent and the other asymptotic. The selection of one of these two on the basis of speed and accuracy depends on the values of the arguments as described in the following discussion.

\section{The Calculation of Psi}

\section{a. Convergent Series}

The development of the uniformly convergent series for $\psi$ is considered first. By writing the factor $\frac{1}{1+\mathrm{Y}^{2}}$ as the Laplace transform of its inverse Laplace transform, we have

$$
\psi(\mathrm{X}, \theta)=\frac{1}{\sqrt{4 \pi \theta}} \int_{-\infty}^{\infty} \exp \left[-(\mathrm{Y}-\mathrm{X})^{2} / 4 \theta\right] \cdot \mathrm{dY} \int_{-\infty}^{\infty} \exp \left[-\left(1+\mathrm{Y}^{2}\right) \mathrm{t}\right] \mathrm{dt}
$$

Since the last integral converges uniformly, we may interchange the order of integration and then complete the square on $\mathrm{Y}$. Thus,

$\psi(\mathrm{X}, \theta)=\frac{1}{\sqrt{4 \pi \theta}} \int_{0}^{\infty} \exp -\left[\mathrm{t}+\frac{\mathrm{X}^{2}}{4 \theta}\left(1-\frac{1}{1+4 \theta t}\right) \mathrm{dt} \int_{-\infty}^{\infty} \exp \left[-\left(\frac{1+4 \theta t}{4 \theta}\right)\left(\mathrm{Y}-\frac{\mathrm{X}}{1+4 \theta t}\right)^{2}\right] \mathrm{dY}\right.$

Now if

$$
\begin{gathered}
\mathrm{z} \equiv\left(\frac{1+4 \theta t}{4 \Theta}\right)^{1 / 2}\left(\mathrm{Y}-\frac{\mathrm{X}}{1+4 \theta t}\right) \\
\psi(\mathrm{X}, \Theta)=\int_{0}^{\infty}(1+4 \theta)^{-1 / 2} \exp \left[-\left(\mathrm{t}+\frac{\mathrm{X}^{2}}{4 \Theta}-\frac{\mathrm{X}^{2}}{(1+4 \Theta t) 4 \Theta}\right)\right] \mathrm{dt} \int_{-\infty}^{\infty} \frac{1}{\sqrt{\pi}} \mathrm{e}^{-\mathrm{z}^{2}} \mathrm{dz}
\end{gathered}
$$

The last factor is unity. On changing the variable of integration to $u \equiv(1+4 \Theta t) / 4 \Theta$, $\psi(\mathrm{X}, \theta)$ may be expressed as

$$
\begin{aligned}
\psi(\mathrm{X}, \theta) & =\exp \left[-\left(\mathrm{X}^{2}-1\right) / 4 \theta\right](4 \theta)^{-1 / 2} \int_{1 / 4 \theta}^{\infty} \mathrm{u}^{-1 / 2} \mathrm{e}^{-\mathrm{u}} \mathrm{e}^{-\frac{1}{\mathrm{u}}\left(\frac{\mathrm{X}}{4 \theta}\right)^{2}} \mathrm{du} \\
= & (4 \Theta)^{-1 / 2}\left[\exp \left(-\left(\mathrm{X}^{2}-1\right) / 4 \theta\right)\right] \int_{1 / 4 \theta}^{\infty} \mathrm{e}^{-\mathrm{u}} \mathrm{u}^{-1 / 2} \sum_{\mathrm{i}=0}^{\infty} \frac{1}{\mathrm{i} !}\left[\frac{\mathrm{x}^{2}}{4 \theta} \frac{1}{\mathrm{u}}\right]^{\mathrm{i}} \mathrm{du} \\
& -73-
\end{aligned}
$$


Clearly the series may be integrated termwise. Define

$$
\begin{aligned}
& \Gamma_{0}(\theta)=\frac{e^{1 / 4 \theta}}{\sqrt{4 \theta}} \int_{1 / 4 \theta}^{\infty} u^{-1 / 2} e^{-u} d u \\
& \Gamma_{i}(\theta)=\frac{e^{1 / 4 \theta}}{\sqrt{4 \theta}} \int_{1 / 4 \theta}^{\infty} u^{-\frac{2 i+1}{2}} e^{-u} d u
\end{aligned}
$$

Then

$$
\Gamma_{i+1(\theta)}=\frac{2}{2 i+1}\left[(4 \theta)^{i}-\Gamma_{i}(\theta)\right]
$$

and

$$
\psi(X, \theta)=e^{-\frac{x^{2}}{4 \theta}} \sum_{i=0}^{\infty} \frac{1}{i !}\left(\frac{x}{4 \theta}\right)^{2 i} \Gamma_{i}(\theta)
$$

The term $\Gamma_{0}(\theta)$ is tabulated using the extensive tables of the incomplete gamma function. With $\Gamma_{0}(X, \theta)$ and equations $(149)$ and $(150)$, one may calculate $\psi(X, \theta)$ to any accuracy desired.

\section{b. Asymptotic Series}

To develop the asymptotic series we express $\psi(X, \theta)$ in slightly different

form.

$$
\psi=\frac{1}{\sqrt{\pi}} \int_{-\infty}^{\infty} \frac{e^{-u^{2}} d u}{1+(\lambda u+x)^{2}}
$$

where

$$
\lambda=\sqrt{4 \Theta} \text { and } u \equiv \frac{(\mathrm{Y}-\mathrm{X})}{\sqrt{4 \theta}}
$$

and expand in a Taylor series with $\lambda$ as argument. Thus,

$$
\begin{array}{r}
\psi(X, \lambda)=\left.\sum_{i=0}^{\infty} \frac{\lambda^{i}}{i !} \frac{\partial^{i} \psi(X, \lambda)}{\partial \lambda^{i}}\right|_{\lambda=0} \\
-74-
\end{array}
$$


Now the problem is to find

$$
\left.\frac{\partial^{i} \psi(x, \lambda)}{\partial \lambda^{i}}\right|_{\lambda=0}
$$

Clearly

$$
\psi(\mathrm{X}, 0)=\frac{1}{1+\mathrm{X}^{2}}
$$

\section{Consider}

$$
\begin{aligned}
F(X, \lambda) & \equiv \frac{1}{1+(\lambda u+X)^{2}}=\cdot \frac{\frac{1}{u}}{\frac{1}{u^{2}}+\left(\lambda+\frac{x}{\dot{u}}\right)^{2}} \frac{1}{u} \\
& =\frac{1}{u} \int_{0}^{\infty} e^{-\left(\lambda+\frac{x}{u} \mid t\right.} \sin \frac{t}{u} d t \\
& =\int_{0}^{\infty} e^{-(u \lambda+X) z} \sin z d z
\end{aligned}
$$

where

$$
z \equiv \frac{t}{u}
$$

Then

$$
\frac{\partial^{i} F}{\partial \lambda^{i}}=(-u) \int_{0}^{i} z^{i} e^{-(u \lambda+X) z} \sin z d z
$$

and

$$
\left.\frac{\partial^{i} \psi}{\partial \lambda^{i}}\right|_{\lambda=0}=\frac{1}{\sqrt{\pi}} \int_{-\infty}^{\infty}(-u)^{i} e^{-u^{2} d u} \int_{0}^{\infty} z^{i} e^{-X z} \sin z d z
$$

It is evident that for $\mathrm{i}$ odd

$$
\left.\frac{\partial^{1} \psi(\mathrm{X}, \lambda)}{\partial \lambda^{i}}\right|_{\lambda=0}=0
$$


When $i$ is even

$$
\int_{-\infty}^{\infty}(-u)^{i} e^{-u^{2} d u}=\frac{\sqrt{\pi} \cdot 1 \cdot 3 \cdot 5 \ldots(i-1)}{2^{i}}
$$

and $^{*}$

$$
\int_{0}^{\infty} z^{i} e^{-X z} \sin z d z=\Gamma(i+1)\left(x^{2}+1\right)^{-\frac{i+1}{2}} \sin \left[(i+1) \sin ^{-1}\left(1+x^{2}\right)^{-1 / 2}\right]
$$

Using the above results we may write

$\psi(\mathrm{X}, \theta)=\sum_{\mathrm{k}=0}^{\infty} \frac{1 \cdot 3 \cdot 5 \ldots(2 \mathrm{k}-1)}{2^{\mathrm{k}}}(4 \theta)^{\mathrm{k}}\left(\mathrm{X}^{2}+1\right)^{-\frac{2 \mathrm{k}+1}{2}} \sin \left[(2 \mathrm{k}+1) \sin ^{-1}\left(\mathrm{X}^{2}+1\right)^{-1 / 2}\right]$

The last series is asymptotic but will serve very well for some ranges of the parameters which complement the ranges over which (150) will converge rapidly.

\section{The Calculation of Chi}

By differentiating the integral form of $\psi(\mathrm{X}, \theta)$ with respect to $\mathrm{X}$, one determines that

$$
\chi(\mathrm{X}, \theta)=2 \theta \frac{\psi}{\mathrm{X}}+\mathrm{X} \psi
$$

Then by differentiating the convergent series for $\psi(\mathrm{X}, \theta)$ one obtains

$$
\chi(X, \theta)=e^{-\frac{x^{2}}{4 \theta}}\left(\frac{x}{4 \theta}\right) \sum_{k=0}^{\infty} \frac{1}{k !}\left(\frac{x^{2}}{4 \theta}\right)^{2 k} \Gamma_{k+1}(\theta)
$$

Similarly by differentiating the asymptotic series for $\psi(\mathrm{X}, \Theta)$ one finds

$\chi(\mathrm{X}, \theta)=\sum_{\mathrm{k}=0}^{\infty}\left|\frac{1.3 .5 \ldots(2 \mathrm{k}-1)}{2^{\mathrm{k}}}\right|(4 \Theta)^{\mathrm{k}}\left(\mathrm{x}^{2}+1\right)^{\frac{-2 \mathrm{k}+1}{2}}(2 \mathrm{k}+1) \sin ^{-1}\left(\mathrm{x}^{2}+1\right)^{-1 / 2}$

There is no need to calculate the trigonometric functions or the square root since

* D. Bierens DeHann, Nouvelles Tables d'Integrales Definies, Hafner Publishing Co., New York (1957). 


$$
\begin{aligned}
\frac{1}{\sqrt{x^{2}+1} \sin \left[(2 i+1) \sin ^{-1}\left(x^{2}+1\right)^{-\frac{1}{2}}\right]} & =\frac{1}{x^{2}+1} \text { when } i=0 \\
& =\frac{1}{\sqrt{x^{2}+1}}\left\{\sin \left[(2 i-1) \sin ^{-1}\left(x^{2}+1\right)^{-\frac{1}{2}}\right] \frac{x^{2}}{x^{2}+1}\right. \\
& \left.+\frac{2 x}{x^{2}+1} \cos \left[(2 i-1) \sin ^{-1}\left(x^{2}+1\right)^{-\frac{1}{2}}\right]\right\} \\
& \text { when } i \neq 0
\end{aligned}
$$

and

$$
\begin{aligned}
& \frac{1}{\sqrt{x^{2}+1} \cos \left[(2 i+1) \sin ^{-1}\left(x^{2}+1\right)^{-\frac{1}{2}}\right]=} \frac{x}{x^{2}+1} \text { when } i=0 \\
&=\frac{1}{\sqrt{x^{2}+1}}\left\{\frac{x^{2}}{x^{2}+1} \cos \left[(2 i-1) \sin ^{-1}\left(x^{2}+1\right)^{-\frac{1}{2}}\right]\right. \\
&\left.-\frac{2 x}{x^{2}+1} \sin \left[(2 i-1) \sin ^{-1}\left(x^{2}+1\right)^{-\frac{1}{2}}\right]\right\} \\
& \text { when } i \neq 0
\end{aligned}
$$

Hence one may calculate Psi and Chi simultaneously by the asymptotic series.

\section{Numerical Procedure}

The program uses the asymptotic series for values of $\mathrm{X}$ and $\theta$ for which it will give the accuracy desired and uses the convergent series elsewhere. The two regions are separated by a parabola whose coefficients must be determined experimentally. Moreover, a parabola which works for Psi may be, used for Chi although the convergent series will be "overworked" in the latter case. 
The convergent series are

where

$$
\psi^{a}(x, \theta)=e^{-\frac{x^{2}}{4 \theta}} \sum_{q=0}^{Q}\left(\frac{x^{2}}{4 \theta}\right)^{2 q} \alpha(4 \theta)
$$

$$
\alpha_{q}(4 \theta)=\frac{\Gamma_{q}(4 \theta)}{q !}
$$

$\Gamma_{0}(4 \theta)$ is tabulated in a table of approximately 50 values with its arguments, and

$$
\begin{aligned}
& \Gamma_{q+1}(4 \theta)=\frac{2}{2 q+1}\left[(4 \theta)^{q}-\Gamma_{q}(4 \theta)\right] \\
& X^{a}(x, \theta)=\frac{x}{4 \theta} e^{-\frac{x^{2}}{4 \theta}} \sum_{q=0}^{Q}\left(\frac{x^{2}}{4 \theta}\right)^{2 q} \alpha_{q}^{\prime}(4 \theta)
\end{aligned}
$$

where

$$
\alpha_{q}^{\prime}(4 \theta)=\frac{\Gamma_{q+1}(4 \Theta)}{q !}
$$

$Q$, the value of $q$ at which the series (167) and (167A) are terminated, is defined as the value at which

$$
\left\{\left[{\left.\frac{x}{4 \theta}\right)^{2 q}}^{2} \alpha_{q}(4 \theta)\right] / \sum_{i=0}^{Q}\left(\frac{x}{4 \theta}\right)^{2 q} \alpha_{q}(4 \theta)\right\} \leq 0.0156
$$

The asymptotic series are

where

$$
\psi^{b}(X, \theta)=\sum_{q=0}^{4} \frac{1 \cdot 3 \ldots(2 q-1)}{2^{q}} 4 \Theta^{q}\left(x^{2}+1\right)^{-\frac{2 q+1}{2}} \sin [(2 q+1) \alpha]
$$

$$
\begin{gathered}
\sin \alpha=\left(\mathrm{x}^{2}+1\right)^{-\frac{1}{2}}, \sin 2 \alpha=\frac{2 \mathrm{x}}{\mathrm{x}^{2}+1} ; \cos 2 \alpha=\frac{\mathrm{x}^{2}-1}{\mathrm{x}^{2}+1} \\
-78-
\end{gathered}
$$


and

$\sin [(n+2) \alpha]=\sin 2 \alpha \cos n \alpha+\cos 2 \alpha \sin n \alpha$

$\chi^{b}(x, \theta)=\sum_{q=0}^{4} \frac{1.3 \ldots(2 q-1)}{-2^{q}}(4 \theta)^{q}\left(x^{2}+1\right)^{-\frac{2 q+1}{2}} \cos [(2 q+1) \alpha]$

where

$$
\cos [(\mathrm{n}+2) \alpha]=\cos 2 \alpha \cos \mathrm{n} \alpha-\sin 2 \alpha \sin \mathrm{n} \alpha
$$

The conditions under which $\psi^{\mathrm{a}}$ is used are:

$$
\begin{aligned}
& 0 \leq \mathrm{X}<1.2 \\
& \text { and } \\
& 4 \Theta \geq 0.275 x^{2}+0.05 \\
& 1.2 \leq \mathrm{X}<10 \\
& \text { and } \\
& 4 \Theta \geq 0.12 \mathrm{x}^{2}+0.28 \\
& 10 \leq \mathrm{X}<100 \\
& \text { and } \\
& 4 \Theta \geq 0.105 \mathrm{x}^{2}+1.75 \\
& 100 \leq X<200 \\
& 4 \Theta \geq 0.095 \mathrm{x}^{2}+100
\end{aligned}
$$

\section{Resonance Equations}

The single level Breit-Wigner formula for the scattering and radiative capture cross sections near a resonance are

$$
\begin{aligned}
& \sigma_{s^{\prime}}\left(E^{\prime}\right)=\pi \pi_{0}^{2} g\left[\frac{\Gamma^{2}+\frac{2 \Gamma \mathrm{n}}{\lambda_{0} \sqrt{\pi}}\left(E^{\prime}-E_{0}\right) \sqrt{\sigma_{p}}}{\left(E^{\prime}-E_{0}\right)^{2}+(\Gamma / 2)^{2}}\right]+\sigma_{p} \\
& \sigma_{c}\left(E^{\prime}\right)=\pi x_{0} x_{g} \frac{\Gamma n^{\Gamma} \gamma}{\left(E^{\prime}-E_{0}\right)^{2}+(\Gamma / 2)^{2}}
\end{aligned}
$$

where $E^{\prime}$ is the energy in the center of mass system, $x_{0}$ is the reduced wave length in the center of mass system corresponding to energy $E_{o}$, and $\sigma_{p}$ is the potential scattering cross section.The energy in the center of mass system is the sum of the energies of the neutron and nucleus obtained using their center of mass system velocities. 


$$
E^{\prime}=\frac{1}{2} \frac{m M}{m+M}|\vec{v}-\vec{v}|^{2}
$$

where $m$ is the neutron mass, $M$ the nuclear mass and $\vec{v}$ and $\vec{V}$ their respective laboratory system velocities.

$$
E^{\prime}=\left[E+\frac{m}{M} E_{N}-V_{p} \sqrt{2 m E}\right] \frac{M}{m+M}
$$

where $E$ is the laboratory system neutron energy, $E_{N}$ that of the nucleus, and $v_{p}$ is the component of the velocity of the nucleus parallel to that of the neutron.

Neglecting the second term in (176)

$$
\begin{aligned}
& V_{p} \approx \frac{E-\frac{\dot{m}+M}{M} E^{\prime}}{\sqrt{2 m E}} \\
& d V_{p} \approx-\frac{m+M}{M} \frac{1}{\sqrt{2 m \bar{E}}} d^{\prime}
\end{aligned}
$$

Assuming a Maxwellian distribution of velocities for the nuclei, the probability of a particular value of $\mathrm{v}_{\mathrm{p}}$ is

$$
\begin{aligned}
P\left(V_{p}\right) d v_{p} & =\sqrt{\frac{M}{2 \pi K T}} \exp \left[-\frac{M v_{p}^{2}}{2 k T}\right] d v_{p} \\
& =\sqrt{\frac{M}{2 \pi K T}} \exp \left[-\frac{M}{2 K T}\left|E-\frac{m+M}{M} E^{\prime}\right|^{2} / 2 m E\right] \frac{M+m}{M \sqrt{2 m E}} d^{\prime} \\
& \equiv P\left(E^{\prime}: \text { E) } d E^{\prime}\right.
\end{aligned}
$$

The effective Doppler broadened cross section, $\sigma_{D}(E)$, is that for which $v \sigma_{D}(E)$ is equal to the actual reaction rate. 


$$
\begin{aligned}
& \sigma_{D}(E)=\frac{1}{v} \int_{0}^{\infty} P\left(E^{\prime}: E\right) \sigma\left(E^{\prime}\right)|\vec{v}-\vec{v}| d E^{\prime} \\
& \begin{array}{l}
=\int_{0}^{\infty} P\left(E^{\prime}: E\right) \quad \sigma\left(E^{\prime}\right) \cdot \sqrt{\frac{M+m}{M} \cdot \frac{E^{\prime}}{E}} d E^{\prime} \\
\left.\int_{0} \sqrt{\frac{M}{4 m \pi K^{\prime} E}}\left(\frac{M+m}{M}\right) \exp \left[-\frac{M}{2 K T} \mid E-\frac{m+M}{M} E^{\prime}\right)^{2} / 2 m E\right]
\end{array} \\
& {\left[\frac { \pi h ^ { 2 } } { 2 \frac { m M } { m + M } \sqrt { E _ { o } E ^ { \prime } } } g \frac { \Gamma _ { n } \Gamma _ { \gamma } } { ( E ^ { \prime } - E _ { o } ) ^ { 2 } + ( \Gamma / 2 ) ^ { 2 } } \left(\left.\frac{m+M}{M}\right|^{\frac{1}{2}}\left(\left.\frac{E^{\prime}}{E}\right|^{\frac{1}{2}}\right] \mathrm{dE}{ }^{\prime}\right.\right.}
\end{aligned}
$$

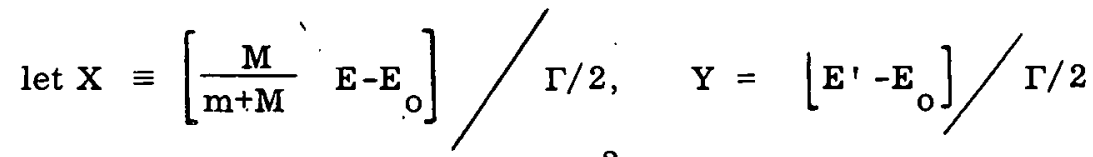

$$
\begin{aligned}
& \theta \equiv \frac{4 \mathrm{mEKT}}{\mathrm{M} \Gamma^{2}} \quad\left(\frac{\mathrm{M}}{\mathrm{m}+\mathrm{M}}\right)^{2}
\end{aligned}
$$

Then

$$
\begin{aligned}
& \sigma_{D_{\cdot \gamma}}(E)=\frac{1}{\sqrt{4 \pi \theta}} \frac{4 \pi h^{2}}{2 m E} g \frac{\Gamma_{n} \Gamma_{0}}{\Gamma^{2}}\left|\frac{m+M}{M}\right|^{3 / 2}\left(\frac{E_{0}}{E}\right)^{\frac{1}{2}} \\
& \int_{\frac{-2 E_{O}}{\Gamma}}^{\infty} \frac{\exp \left[-(X-Y)^{2} / 4 \theta\right]}{1+Y^{2}} d Y
\end{aligned}
$$

Only values of $\mathrm{Y}$ close to $\mathrm{X}$ contribute significantly to the integral in (182); since values of

$\mathrm{X}$ near $-\frac{2 \mathrm{E}}{\Gamma}$ occur only for $E$ near zero and since $\theta$ is proportional to $E$ only a negligible error is introduced by extending the lower limit to $-\infty$. Thus, defining

$$
\sigma_{\gamma_{0}} \equiv \frac{4 \pi \hbar^{2}}{2 m E_{0}} \mathrm{~g}\left(\frac{\mathrm{m}+\mathrm{M}}{\mathrm{M}}\right)^{3 / 2}\left(\frac{\mathrm{E}_{\mathrm{o}}}{\mathrm{E}}\right)^{\frac{1}{2}} \frac{\Gamma_{\mathrm{n} \gamma} \Gamma}{\Gamma^{2}}
$$


and

$$
\begin{aligned}
& \left.\psi \equiv \frac{1}{\sqrt{4 \pi \theta}} \int_{-\infty}^{\infty} \frac{\exp \left[-(X-Y)^{2} / 4 \theta\right.}{1+Y^{2}}\right] d Y \\
& \sigma_{D_{\gamma} \approx \sigma_{0}} \approx(X, \theta)
\end{aligned}
$$

Similarly

$$
\begin{aligned}
& \sigma_{D_{S}}(E)=\frac{1}{\sqrt{4 \pi \theta}} \quad \frac{4 \pi \hbar^{2}}{2 m E_{0}} g \quad \frac{\Gamma_{n}^{2}}{\Gamma^{2}}\left(\frac{m+M}{M}\right)^{3 / 2}
\end{aligned}
$$

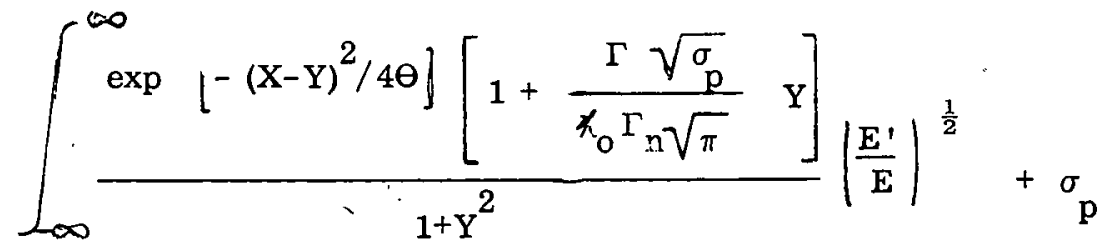

The factor $\left|\frac{E^{\prime}}{E}\right|^{\frac{1}{2}}=\left[\frac{\Gamma}{2 E}(Y-X)+\frac{M}{m+M}\right]^{\frac{1}{2}}$

$$
\approx\left(\frac{M}{m+M}\right)^{\frac{1}{2}}\left[1+\frac{m+M}{M} \frac{\Gamma}{4 E}(Y-X)+\ldots\right]
$$

over the significant portion of the range of $\mathrm{Y}$.

For all cases of interest this is adequately

approximated by $\left(\frac{M}{m+M}\right)^{\frac{1}{2}}$

Defining

$$
\sigma_{s_{0}} \equiv \frac{4 \pi \hbar^{2}}{2 m E_{0}} \mathrm{~g}\left(\frac{m+M}{M}\right)^{2} \quad \frac{\Gamma_{n}^{2}}{\Gamma^{2}}
$$

and

$$
\chi(X, \Theta) \equiv \frac{1}{\sqrt{4 \pi}} \quad \int_{-\infty}^{\infty} \frac{\mathrm{Y} \exp \left[-(\mathrm{X}-\mathrm{Y})^{2} / 4 \Theta\right]}{1+\mathrm{Y}^{2}} \mathrm{dY}
$$

$-82-$ 


$$
\sigma_{D_{s}}=\sigma_{s_{0}} \psi(\mathbf{X}, \theta)+2 \frac{m+M}{M} \sqrt{g \sigma_{p} \sigma_{s}} \chi(X, \theta)+\sigma_{p}
$$

The process of locating the central energies of unresolved resonances described in the main portion of this report assumes a constant density of resonances. In reality there are two phenomena which cause the level density to vary. One is the variation in the density of nuclear levels of zero angular momentum, the second is the appearance of higher angular momentum levels. A linear approximation for the level density as a function of neutron energy has been adopted, as sufficient for the description of cross sections by the resonance parameter tables in RBU:

$$
\mathrm{D}=\mathrm{D}_{\mathrm{o}}\left(1+\mathrm{D}^{*} \mathrm{v}^{2}\right)
$$

A rough approximation to the effect of resonances of angular momentum higher than zero can be obtained by the classical treatment of glancing collisions according to which the highest angular momentum which can contribute to an interaction between a neutron and a nucleus is given by

$$
\ell_{\max }=\mathrm{m} \mathrm{vR} / \hbar
$$

where $R$ is the radius of the nucleus given approximately by $1.45 \times 10^{-13} \mathrm{~A}^{1 / 3} \mathrm{~cm}$. If one assumes that all angular momenta capable, according to (191) of contribution to interactions, produce effectively similar resonances, it appears reasonable to write that the effective density of resonances is given by

$$
\mathrm{D}=\mathrm{D}_{\mathrm{o}}\left(1+\ell^{*} \mathrm{~V}\right)^{2}
$$

where the coefficient $\ell^{*}$ may be estimated as

$$
\ell^{*}=1.45 \times 10^{-13} \cdot \mathrm{A}^{1 / 3} \cdot \mathrm{m} / \mathrm{xi}
$$

in the absence of other information. 
The effect of higher angular momentum resonances, like the effect of the variation of nuclear level density is small enough that these rough approximations are probably satisfactory for reactor calculations but large enough that they should be included. In

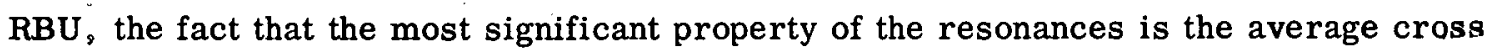
section, the product of the level density and the mean cross section within a resonance. leads to one further approximation. The variation in level density is treated as a variation in the cross section value and the level density is treated as a constant. One source of error associated with this approximation arises from the fact that the effect of cross section value variation is diminished by self-shielding while that of level density variation is not. It appears, however, in view of the small importance of the two phenomena considered here, that the approximate treatment is sufficient.

D. Derivation of the Backward Difference Equations

For a time interval, $\delta$, the backward differences of $\dot{N}$ are defined by

$$
\begin{aligned}
& \nabla^{0}(t)=\dot{N}(t) \\
& \nabla^{r}(t)=\nabla^{r-1}(t)-\nabla^{r-1}(t-\delta)
\end{aligned}
$$

Equation (195) may be solved for the values of $\dot{N}$ at preceding time steps:

$$
\begin{aligned}
& \dot{N}(t)=\nabla^{0}(t) \\
& \dot{N}(t-\delta)=\nabla^{0}(t)-\nabla^{1}(t) \\
& \dot{N}(t-n \delta)=\sum_{r=0}^{n}(-1)^{r}\left(\begin{array}{r}
n \\
r
\end{array} \nabla^{r}(t)\right.
\end{aligned}
$$

Equation (196) yields values of $\mathrm{N}$ at one point for each of the $\mathrm{R}$ backward differences available. The value of $\mathrm{N}$ at an arbitrary time, $t \mathbf{t}$, may then be approximated by a power series

$$
\dot{N}\left(t^{\prime}\right)=\sum_{r=0}^{R} A_{r}\left(t^{\prime}-t\right)^{r}
$$

Combining equations (196) and (197), one obtains a set of $\mathrm{R}$ simultaneous algebraic equations in the $\mathrm{A}_{\mathrm{r}}$. 


$$
\sum_{r=0}^{R} A_{r}(n \delta)^{r}=\sum_{i=0}^{-n}(-1)^{i}\left(\begin{array}{r}
-n \\
i
\end{array}\right) \nabla^{i} \text { (t) }
$$

For a given value of $R$, these may be solved by elimination to give

$$
\begin{aligned}
& \mathrm{A}_{1}=\frac{1}{\delta}\left[\nabla^{1}+\frac{1}{2} \nabla^{2}+\frac{1}{3} \nabla^{3}+\frac{1}{4} \nabla^{4}+\frac{1}{5} \nabla^{5}+\ldots\right. \\
& \mathrm{A}_{2}=\frac{1}{\delta}\left[\frac{1}{2} \nabla^{2}+\frac{1}{2}+\nabla^{3}+\frac{11}{24} \nabla^{4}+\frac{5}{12} \nabla^{5}+\ldots\right. \\
& \mathrm{A}_{3}=\frac{1}{\delta^{3}}\left[\frac{1}{6} \nabla^{3}+\frac{1}{4} \nabla^{4}+\frac{7}{24} \nabla^{5}+\ldots\right. \\
& \mathrm{A}_{4}=\frac{1}{\delta}\left[\frac{1}{24} \nabla^{4}+\frac{1}{21} \nabla^{5}+\ldots\right. \\
& \mathrm{A}_{5}=\frac{1}{\delta}\left[\frac{1}{120} \nabla^{5}+\ldots\right. \\
& \text { et cetera }
\end{aligned}
$$

For smaller values of $\mathrm{R}$ the same solutions hold if the $\nabla^{\mathrm{r}}$ for $\mathrm{r}>\mathrm{R}$ are set equal to zero.

By substituting the appropriate values of $t^{\prime}$ in (197) and using the coefficients of (199) for $\mathrm{R}=3$, the interpolation equation for $\mathrm{N}$ in the body of this report is obtained. Similarly . by integrating (197) and making the same substitution the prediction and correction formulae are found.

\section{E. Derivation of the Diffusion Difference Equations}

The diffusion equation in one dimension is.

$$
f(r)=-\frac{1}{r^{\rho}} \quad \frac{\partial}{\partial r} D(r) \quad r^{\rho} \quad \frac{\partial}{\partial r} \phi(r)-H(r)+T(r) \phi(r)=0
$$

To obtain an equation involving values of the neutron flux at the mesh points $r=r_{0}, r_{1} \ldots r_{N}$ only, one multiplies Equation (200) by $\mathbf{r}^{\rho}$ and integrates from

$$
\begin{aligned}
\frac{r_{n-1}+r}{2} \equiv r_{n-1 / 2} & \text { to } \frac{r_{n}+r}{2} \equiv r_{n+1 / 2} \\
& -85-
\end{aligned}
$$


Between $r_{n-1 / 2}$ and $r_{n}$ and between $r_{n}$ and $r_{n+1 / 2}$, the nuclear constants, $D$ and $T$, do not vary. Then assuming that $\phi$ and $H$ vary linearly between mesh points:

$$
\begin{aligned}
& \left.\left.\int_{r_{n-\frac{1}{2}}}^{r_{n+\frac{1}{2}}} r^{\rho} f(r) d r=-D_{n-} r^{\rho} \frac{\partial}{\partial r} \phi(r)\right]_{r_{n-\frac{1}{2}}}^{r_{n}}-D_{n+} r^{\rho} \frac{\partial}{\partial r} \phi(r)\right]_{r_{n}}^{r_{n+\frac{1}{2}}} \\
& +T_{n-} \int_{r_{n-\frac{1}{2}}}^{r_{n+\frac{1}{2}}} r^{\rho}\left[\phi_{n}+\frac{\phi_{n}-\phi_{n-1}}{\Delta r_{n-}}\left(r-r_{n}\right)\right] \quad d r \\
& +T_{n+} \int_{r_{n}}^{r_{n+\frac{1}{2}}} r^{\rho}\left[\phi_{n}+\frac{\phi_{n+1}-\phi_{n}}{\Delta r_{n+}}\left(r-r_{n}\right)\right] d r \\
& -\int_{r_{n-\frac{1}{2}}}^{r_{n}} r^{\rho}\left[H_{n-}+\frac{H_{n-}-H_{n-1}^{+}}{\Delta r_{n-}}\left(r-r_{n}\right)\right] d r \\
& -\int_{r_{n}}^{r_{n+\frac{1}{2}}} r^{\rho}\left[H_{n+} \frac{H_{n+1-}-H_{n+}}{\dot{\Delta}^{r}}\left(r-r_{n}\right)\right] \quad d r=0
\end{aligned}
$$

In (202) $H(r)$ is permitted to be discontinuous at mesh points while $\phi$ has been assumed to be continuous. 


$$
\begin{aligned}
& \int_{r_{n-1 / 2}}^{r_{n+1 / 2}} r^{\rho} f(r) d r=-D_{n-} \frac{\phi_{n}-\phi_{n-1}}{\Delta r_{n-}}\left[r_{n}^{\rho}-\left|r_{n}-\frac{1}{2} \Delta r_{n-}\right|^{\rho}\right] \\
& -\mathrm{D}_{\mathrm{n}+} \frac{\phi_{\mathrm{n}+1}-\phi_{\mathrm{n}}}{\Delta \mathrm{r}_{\mathrm{n}+}}\left[\left(\mathrm{r}_{\mathrm{n}}+\frac{1}{2} \Delta \mathrm{r}_{\mathrm{n}+} \|^{\rho}-\mathrm{r}_{\mathrm{n}}^{\rho}\right]\right. \text {. } \\
& \left.+T_{n-}\left[\frac{1}{\rho+1} r^{\rho+1} \phi_{n}+\frac{\phi_{n}-\phi_{n-1}}{\Delta r_{n-}} \mid \frac{1}{\rho+2} r^{\rho+2}-\frac{1}{\rho+1} r_{n} r^{\rho+1}\right)\right]_{n-1 / 2}^{r_{n}} \\
& \left.+T_{n+}\left[\frac{1}{\rho+1} r^{\rho+1} \phi_{n}+\frac{\phi_{n+1}-\phi_{n}}{\Delta r_{n t}} \mid \frac{1}{\rho+2} r^{\rho+2}-\frac{1}{\rho+1} r_{n} r^{\rho+1}\right)\right]_{n}^{r_{n}+1 / 2} \\
& \left.-\left[H_{n-1} \frac{1}{\rho+1} r^{\rho+1}+\frac{H_{n-}-H_{n-1}}{\Delta r_{n-}} \mid \frac{1}{\rho+2} r^{\rho+2}-\frac{1}{\rho+1} r_{n} r^{\rho+1}\right)\right]_{n-1 / 2}^{r_{n}} \\
& -\left[\mathrm{H}_{\mathrm{n}+} \frac{1}{\rho+1} \mathrm{r}^{\rho+1}+\frac{\mathrm{H}_{\mathrm{n}+1-\mathrm{H}_{\mathrm{n}}}}{\Delta \mathrm{r}_{\mathrm{n}}}\left(\frac{1}{\rho+2} \mathrm{r}^{\rho+2}-\frac{1}{\rho+1} \mathrm{r}_{n} r^{\rho+1}\right)\right]_{\mathrm{r}_{\mathrm{n}}}^{\mathrm{r}_{\mathrm{n}+1 / 2}}=0 \text { (203) }
\end{aligned}
$$

On substituting limits and collecting terms, one finds

$$
\begin{aligned}
& -a_{n} \phi_{n-1}+b_{n} \phi_{n}-c_{n} \phi_{n+1}+a_{n}^{\prime} H_{n-1}-b_{n}^{\prime} H_{n-}-b_{n}^{\prime \prime} H_{n+}+c_{n}^{\prime} H_{n+1}=0 \\
& a_{n}=D_{n-} \frac{r_{n-1 / 2}}{\Delta r_{n-}}+T_{n-} a_{n}^{\prime} \\
& b_{n}=a_{n}+c_{n}+T_{n-} \cdot \frac{r_{n}^{\rho+1}-r_{n-1 / 2}^{\rho+1}}{\left(\rho^{+1)}\right.}-T_{n+} \frac{r_{n}^{\rho+1}-r_{n+1 / 2}^{\rho+1}}{\rho^{+1}} \\
& c_{n}=\frac{r_{n+1 / 2}^{\rho}}{\Delta r_{n+}}+T_{n+} c_{n}^{\prime} \\
& a_{n}^{\prime}=\frac{r_{n}^{\rho+2}-r_{n-1 / 2}^{\rho+2}}{\rho^{++2 \Delta r_{n-}}-\frac{r_{n}^{\rho+2}-r_{n} r_{n-1 / 2}^{\rho+1}}{(\rho+1) \Delta r_{n-}}} \\
& c_{n}^{\prime}=\frac{r_{n}^{\rho+2}-r_{n+1 / 2}^{\rho+2}}{(\rho+2) \Delta r_{n+}}-\frac{r_{n}^{\rho+2}-r_{n} r_{n+1 / 2}^{\rho+1}}{(\rho+1) \Delta r_{n+}} .
\end{aligned}
$$




$$
\begin{aligned}
& b_{n}^{\prime}=a_{n}^{\prime+} \frac{r_{n}^{\rho+1}-r_{n-1 / 2}^{\rho+1}}{\rho^{+1}} \\
& b_{n}^{\prime \prime}=c_{n}^{\prime}-\frac{r_{n}^{\rho_{j}^{+1}}-r_{n+1 / 2}^{\rho+1}}{\rho+1}
\end{aligned}
$$

The approximation

$$
\mathrm{r}_{\mathrm{n}}^{\rho}-\mathrm{r}_{\mathrm{n}-1 / 2}^{\rho} \approx \frac{1}{2} \rho \mathrm{r}_{\mathrm{n}}^{\rho-1} \cdot \Delta \mathrm{r}_{\mathrm{n}-}
$$

is accurate to first order in $\Delta r$. With this approximation

$$
a_{n}^{\prime}=c_{n}^{\prime}=0
$$

and (205) reduces to

$$
-a_{n} \phi_{n-1}+b_{n} \phi_{n}-c_{n} \phi_{n+1}-d_{n-} H_{n-}-d_{n+} H_{n+}=0
$$

where

$$
\begin{aligned}
& a_{n}=\left(D_{n-}\right) \frac{r_{n-1 / 2}^{\rho}}{\Delta r_{n-}} \\
& b_{n}=a_{n}+c_{n}-T_{n}-r_{n}^{\rho} \frac{\Delta r_{n-}}{2}-T_{n+} r_{n}^{\rho} \frac{\Delta r_{n+}}{2} \\
& c_{n}=\left(D_{n+}\right) \frac{r_{n+1 / 2}^{\rho}}{\Delta r_{n+}} \\
& d_{n-}=r_{n}^{\rho} \frac{\Delta r_{n-}}{2}, \quad d_{n+}=r_{n}^{\rho} \frac{\Delta r_{n+}}{2}
\end{aligned}
$$

$-88^{\prime}-$ 


\section{TAPE LAYOUTS}

A. RBU INSTRUCTION TAPE, TAPE 1

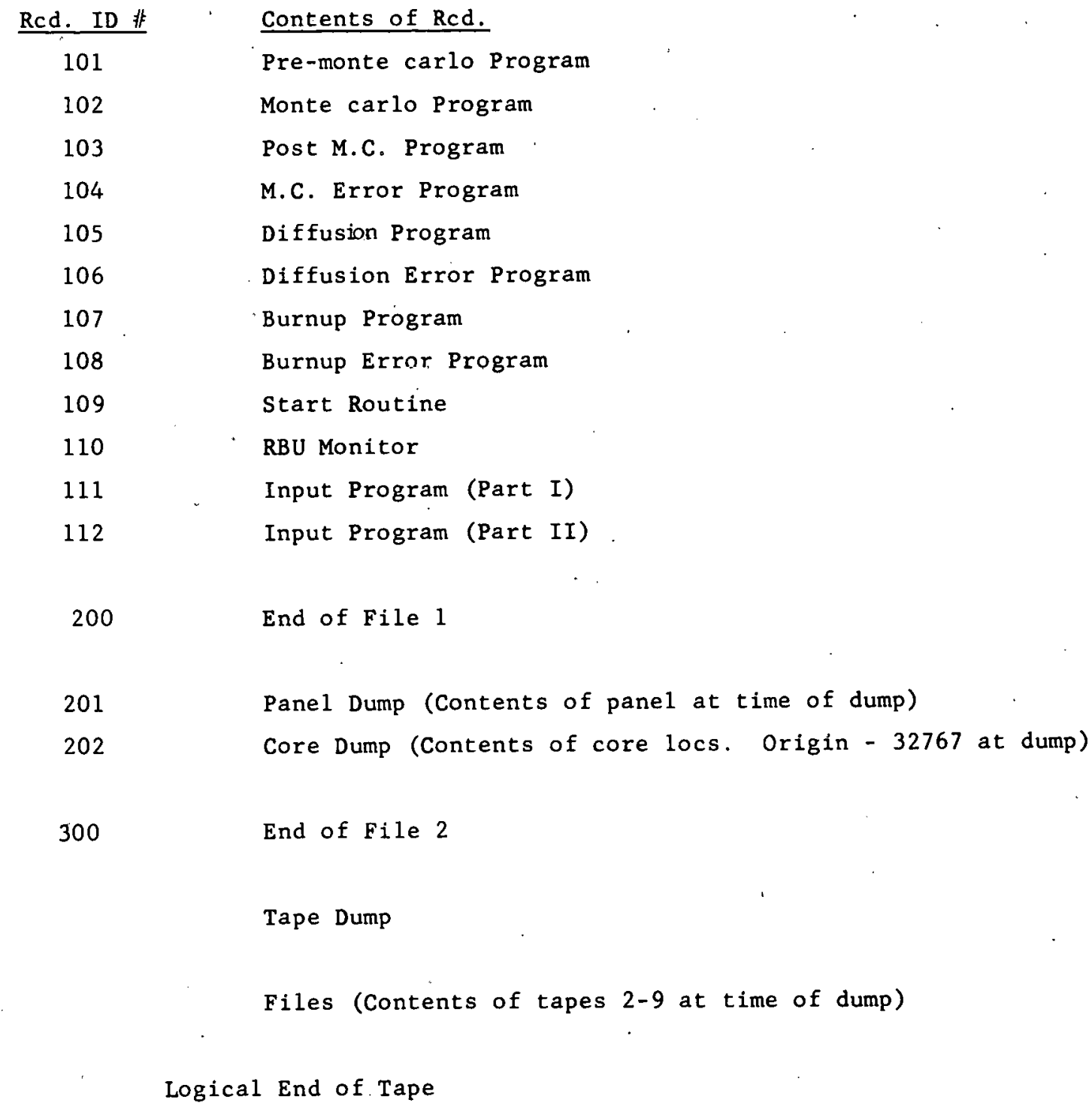


Note: Each record of files 1 and 2 begins with a 3-word ID sequence as follows:

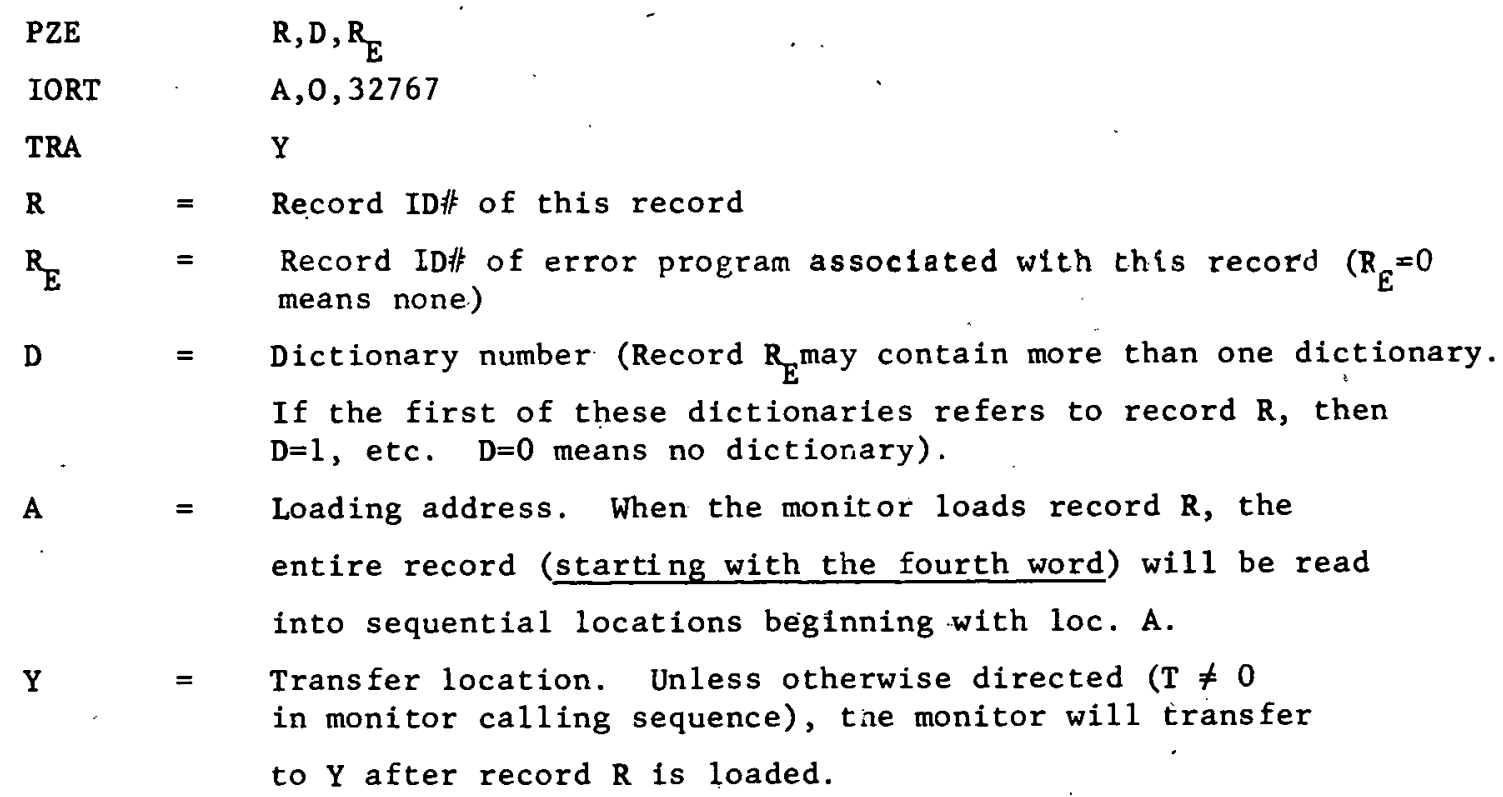


B. BASIC LIBRARY (PRELIMINARY)

Record 1

$A_{1}$

(4)

D'

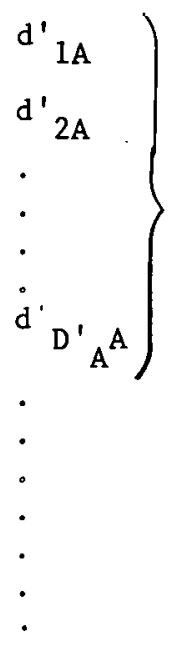

$S^{\prime}$

$\mathbf{s}^{\prime}$

$P_{\text {in } 1 s}$

$\mathrm{P}_{\text {in }}$

$\cdot$

-

$\dot{\mathrm{P}}_{\text {in }}$

$\Delta v^{2}$ in

$\Delta v^{2}{ }_{\text {in }}$ ls

-

$\Delta v^{2}{ }_{\text {in }}$
Isotope Name

0 if isotope does not contain any ranges for which $\delta=4$

Number of direct and indirect descendants of isotope A

Names of direct and indirect descendants of isotope A.

Repeat for each isotope in library

\section{Record 2}

Number of tables of inelastic spectra

Inelastic Spectrum Table Number

17 probabilities for the following list of $\Delta v^{2}$ for table s.

$$
\mathrm{P}_{\text {in }_{1}}=1, \mathrm{P}_{\text {in }_{17}}=0 \text {. }
$$

17 changes in $v^{2}$ corresponding to preceding list of $P_{\text {in }}$ for table $s$ in order of decreasing $v^{2}$. 
$\left.\begin{array}{l}\cdot \\ \cdot \\ \cdot \\ \cdot\end{array}\right\}$

Repeat for each inelastic spectrum

e

Number of tables of resonance parameters

$\rho$

Resonance parameter table number

${ }_{1}^{E_{p}}$

Maximum energy for unresolved resonances

$\mathrm{E}_{2}$

$E_{3}$

Minimum energy for unresolved resonances

Minimum energy for resolved resonances

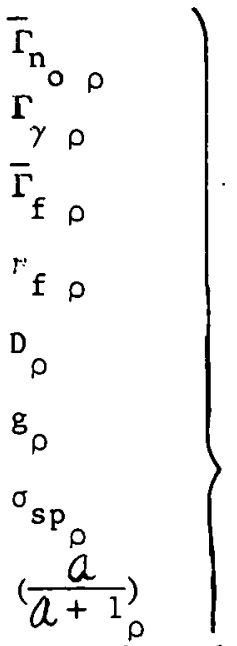

Resonance parameters for unresolved resonances for table $\rho$. A value of $\mathrm{D}_{\rho}$ of zero will cause the monte carlo program to bypass the use of unresolved resonances.

$5.563 \times 10^{3}\left(\frac{a+1}{a}\right)^{2}$

$3.6006 \times 10^{6}\left(\frac{a+1}{a}\right)^{3 / 2}$

$\mathrm{D}_{0}{ }^{*}$
$\ell_{0}^{*}$
$\sigma_{\mathrm{C}}^{*}$
$\sigma_{\mathrm{f}^{*}}$
$\mathrm{~N}_{\mathrm{E}}$

$\left.\begin{array}{l}\mathrm{E}_{\mathrm{o}_{\tau \rho}} \\ \Gamma_{\tilde{\tau} \rho} \\ \dot{\Gamma}_{\gamma_{\tau} \rho} \rho \\ \dot{\mathrm{g}}_{\tau} \rho\end{array}\right\}$

Number of resolved resonances in table $\rho$

Resonance parameters for the $r^{\text {til }}$ resolved resonance in the $\rho^{\text {th }}$ table of resonance parameters in order of increasing $\tau$ 


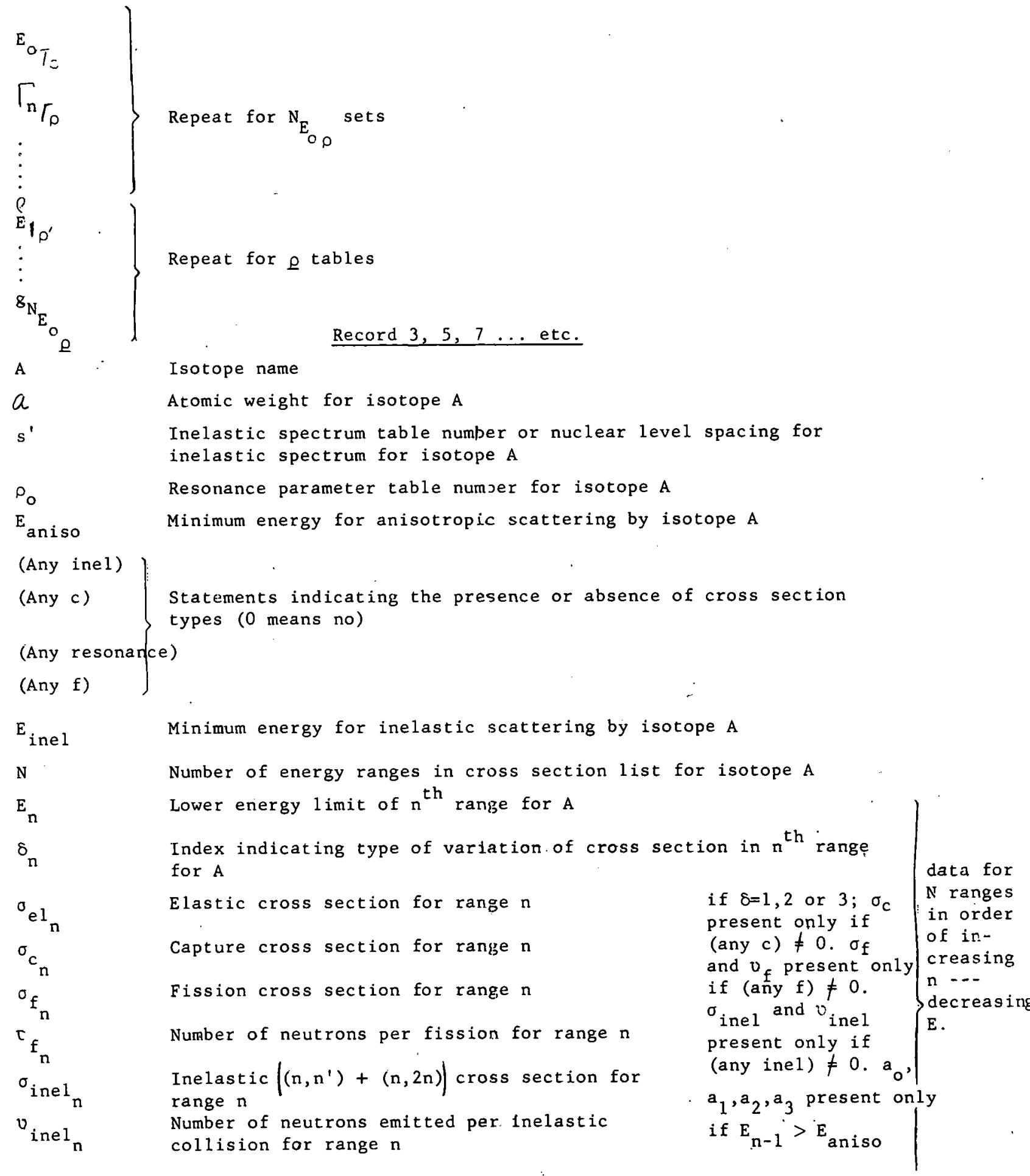




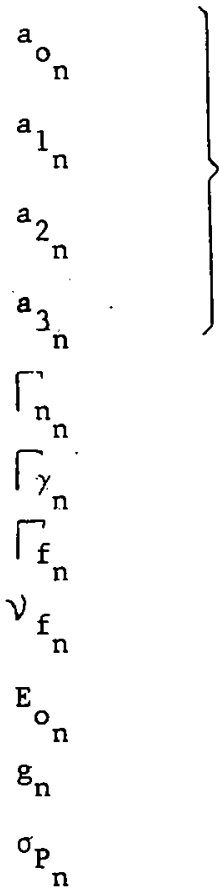

Anisotropic scattering coefficients

for range $n$

Neutron width for range $n$

Gamna width for range $n$

Fission width for range $n$

Number of neutrons per fission

$a_{0}, a_{1}, a_{2}, a_{3}$ present only

for range $n$

Peak energy for range $n$

Statistical weight for.range $n$

Potential scattering cross section

for range $n$

(Note: If $\delta=5$ no information is required

to replace the items from $\sigma_{e l}$ to $a_{3}$ above.

Repeat for each energy range

Record 4, 6, $8 \ldots$ etc.

$\mathrm{D}_{\mathrm{A}}$

Number of direct descendants of isotope $A$

$\mathrm{A}_{\mathrm{A}}$

Isotope produced by neutron capture in A

Ignored if (any $c$ ) $=0$ ]

$\mathrm{A}_{1} \quad$ : $\quad$ Isotope produced by $(n, 2 n)$ collisions with $A$

[Ignored if (any inel) $=0]$

$\mathrm{A}_{2}$

Isotope produced by decay with first decay constant in A

(Ignored if $\lambda_{1}=0$ )

A 3 Isotope produced by decay with second decay constant in $A$

(Ignored if $\lambda_{2}=0$ ) 
1

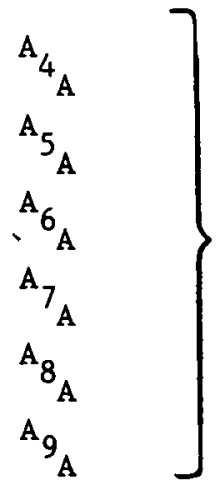

Isotopes produced by fissions in A . : : (Those including and beyond the first for which the corresponding $Y$ is zero will be ignore ed)

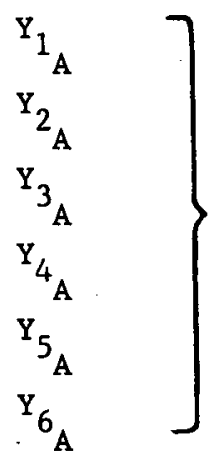

Fractional fission product yields for $\mathrm{A}_{4}$ through $\mathrm{A}_{9}$ $\left.{ }^{\lambda_{1}}{ }_{\mathrm{A}}^{\lambda_{1}}\right\}$ Radioactive decay constant for isotope A

$-95-$ 
C CUMULATIVE CROSS SECTION TAPE (PRELIMINARY)

File 1

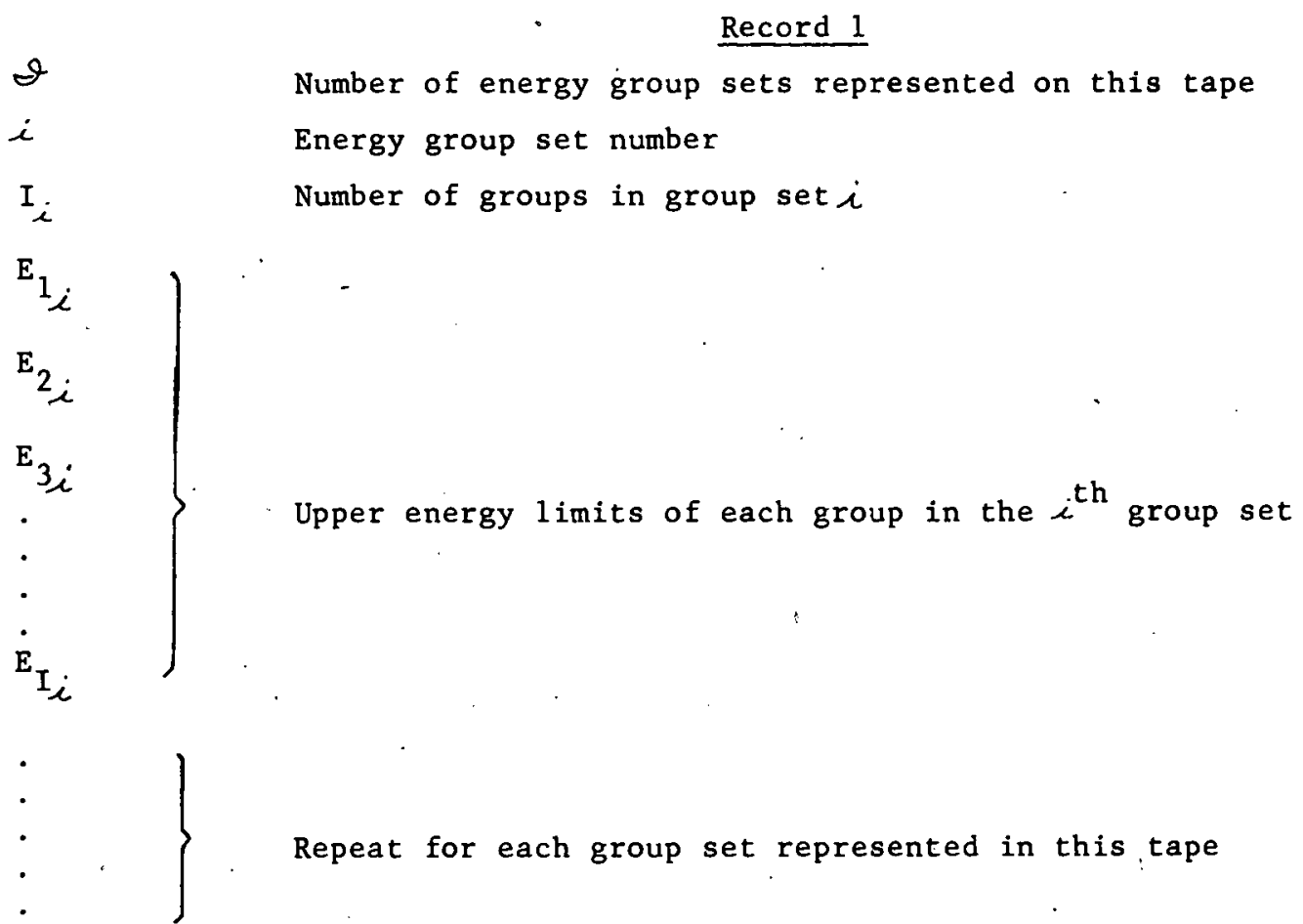

$\underline{\text { Record } 2}$.

$\mathrm{A}_{i}$

Name of first isotope listed in first group ser on this tape.

$\mathrm{T}_{1}$

Temperature of first isotope 1 isted in first group set on this tape or zero if isotope does not contain any ranges with $\delta=4$

$\mathrm{A}_{2}$

Name of second isotope listed in first group set on this tape

$\mathrm{T}_{2}$

Temperature of second isotope listed in first group set on this tape or zero if isutope does not contain any ranges with $\delta=4$

Record 3 through $1+d$

Same as Record 2 for each $i$ 
File 2 through $1+d$ (Each file refers to a group set)

$$
\frac{\text { Record } 1,3,5, \ldots 2 \mathrm{~N}_{i}-1}{\text { (Each record refers to an isotope) }}
$$

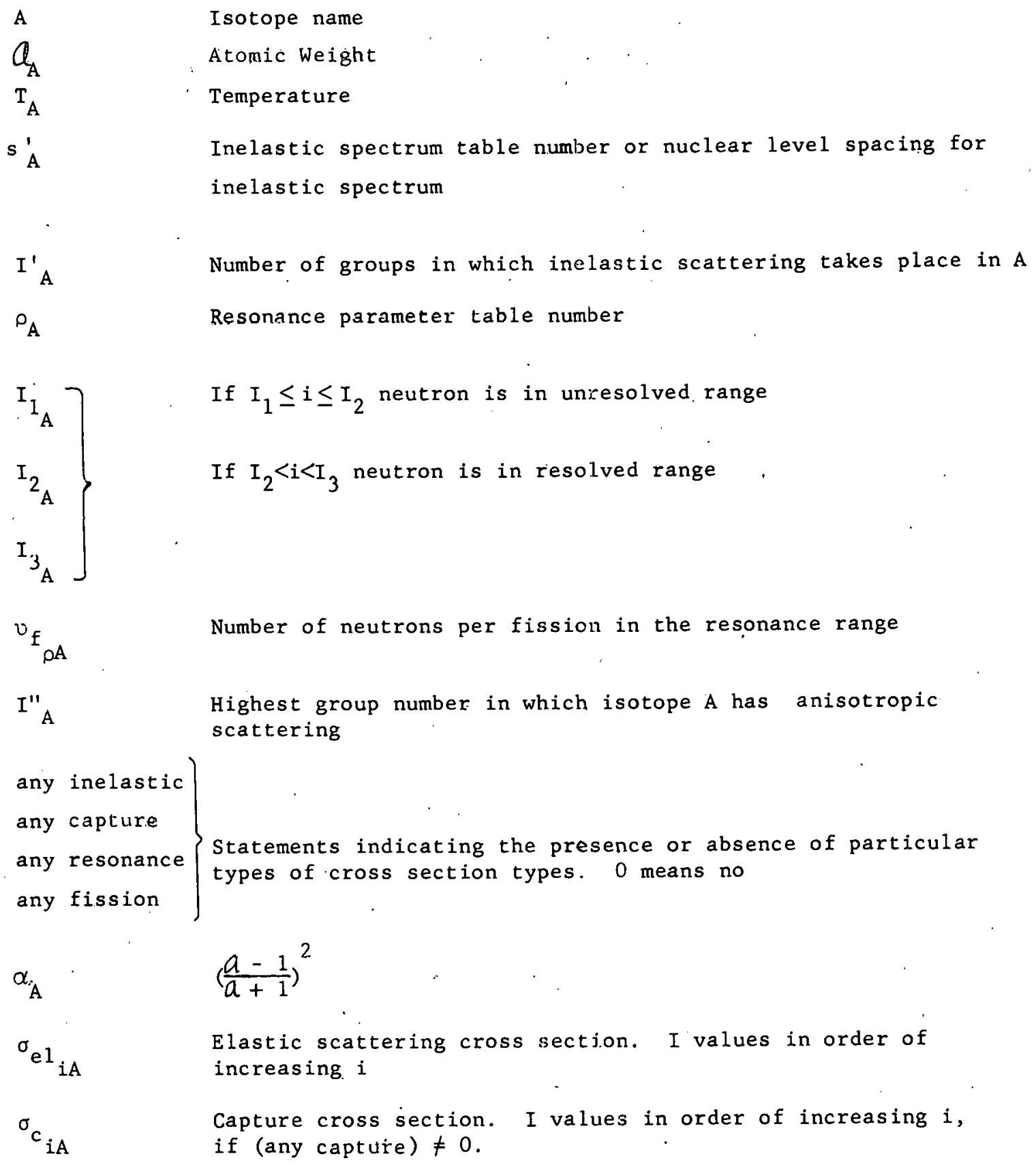




$$
\left.\begin{array}{l}
\sigma_{f_{i A}} \\
\left(v_{f} \sigma_{f}\right)_{i A} \\
\left(\sigma_{n, 2 n}\right)_{i A} \\
\left(v_{i n} \sigma_{i n}\right)_{i A} \\
\sigma_{T_{i A}} \\
a_{o_{i A}} \\
a_{1}{ }_{i A} \\
a_{2}{ }_{i A} \\
a_{3} \\
a_{m i}
\end{array}\right\} .
$$

$$
\mathrm{D}_{\mathrm{A}}
$$$$
\left(A^{\prime}, \mathrm{m}\right)_{1 A}
$$

Fission scattering cross section. I values in order of increasing $i$, if (any fission) $\neq 0$.

Fission neutron emission cross section. I values in order of increasing $i$, if (any fission) $\neq 0$.

$(n, 2 n)$ cross section. I' values if (any inel) $\neq 0$ in order of increasing $i$.

Inelastic neutron emission cross section. ' $I$ ' values if (any ine1) $\neq 0$ in order of increasing $i$.

$\sigma_{e l}+\sigma_{c}+\sigma_{f}+\sigma_{\text {inel }}$. I values

Anisotropic scattering coefficients for group $i$ isotope A. I" sets of $a_{0}, a_{1}, a_{2}, a_{3}$, and $a_{m}$.

Largest value for isotope $A$ in Eroup $i$ of $a_{0}+a_{1} \mu a_{2} \mu^{2}+a_{3} \mu^{3}$

Record 2, 4,6 ...2 $2 A_{i}$

Number of direct descendants of isotope $A$.

Isotope name and mode of production for first direct descendant of $A$.

Descendant in decrement

Mode in address: $0=$ capture

$1=\mathrm{n}, 2 \mathrm{n}$

2 = decay with first decay constant

3 = decay with second decay constant

4 = fission with first fission yield

$5=$ fission with second fission yield

6 = fission with third fission yield

7 = fission with fourth fission yield

8 = fission with fifth fission yield

$9=$ fission with sixth fission yield

Repeat for each descendant of isotope A 


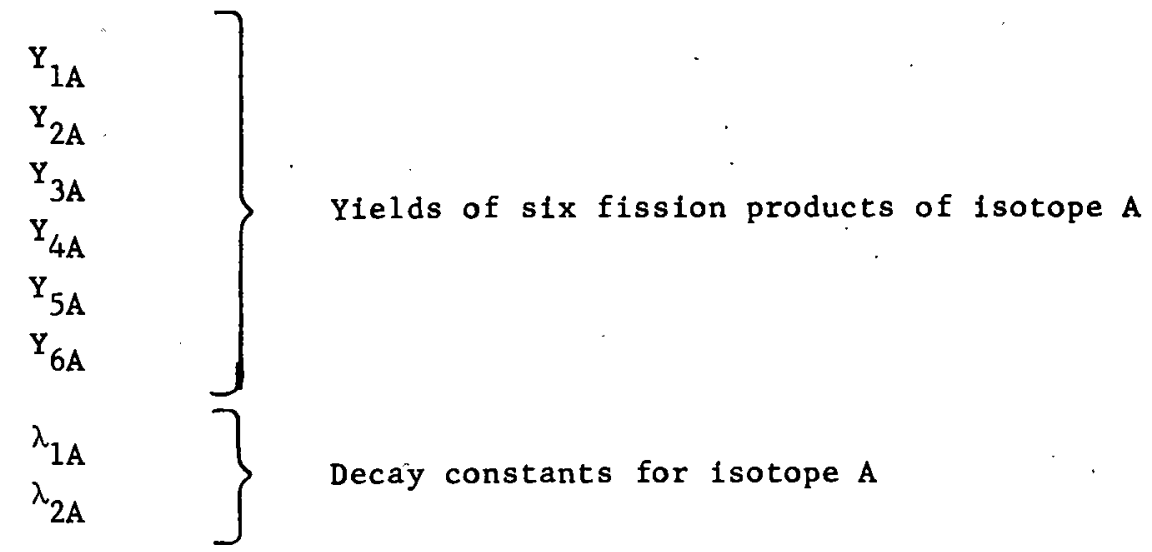


Record 1

$S \quad$ Number of systems

I

$\mathrm{J}$

Number of microscopic groups

Number of macroscopic groups

\section{$\underline{\text { Record } 2}$}

$\left.\begin{array}{c}\mathrm{M}_{1} \\ \mathrm{M}_{2} \\ \cdot \\ \dot{\mathrm{M}}_{\mathrm{S}}\end{array}\right\}$

Highest material number in each system

$\left.\begin{array}{l}\mathrm{Q}_{1} \\ \mathrm{Q}_{2} \\ \dot{\mathrm{Q}}_{\mathrm{S}}\end{array}\right\}$.

$\sum_{m} Q_{m}$ for each system. $Q_{m}$ is the number of isotopes in material m.

Lower energy limit in each microscopic group

Lower energy limit in each macroscopic group

•

$\left.\dot{\mathrm{E}}_{\mathrm{J}}\right)$

$\left.\begin{array}{c}\mathrm{v}_{1} \\ \mathrm{v}_{2} \\ \cdot \\ \mathrm{v}_{\mathrm{I}}\end{array}\right\}$

$Q_{\mathrm{H}}$

Atomic weight criterion for moderation on scattering in monte carlo. 
$\mathbf{P}$

Operating power density (kilowatts $/ \mathrm{cm}^{3}$ ) (volume used is $\frac{1}{\rho+1} R_{N}^{\rho+1}$ )

Record 3

s

t

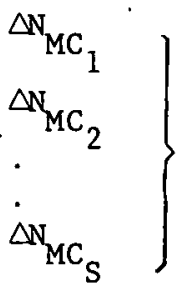

$\left.\begin{array}{l}\pi_{M C_{1}} \\ \pi_{M C_{2}} \\ \pi_{M C_{S}}\end{array}\right\}$

Address of $\mathrm{MC}_{1}$

Address of $\mathrm{MC}_{2}$

$\left.\begin{array}{l}\mathrm{X} \\ \mathrm{X} \\ z \\ \alpha \\ \beta \\ \gamma \\ V \\ t \\ i \\ \mathrm{~h} \\ \mathrm{~s}_{\mathrm{b}} \\ \mathrm{GBqq}^{\prime}\end{array}\right\}$

Number of diffusion time steps since last monte carlo on each system (initially $=\Delta \mathrm{N}_{\mathrm{MC}}$ )

Initially $(1225)_{8}$

Initially $(1227)_{8}$

File 2.

Record 4, $5 \ldots$

Census particle coordinates, 10 to a record (initially not present) 
$\underline{\text { TAPE } 3}$ One file for each system in order of increasing $s$

$$
\text { File } 1,2 \ldots s
$$

\section{Record 1}

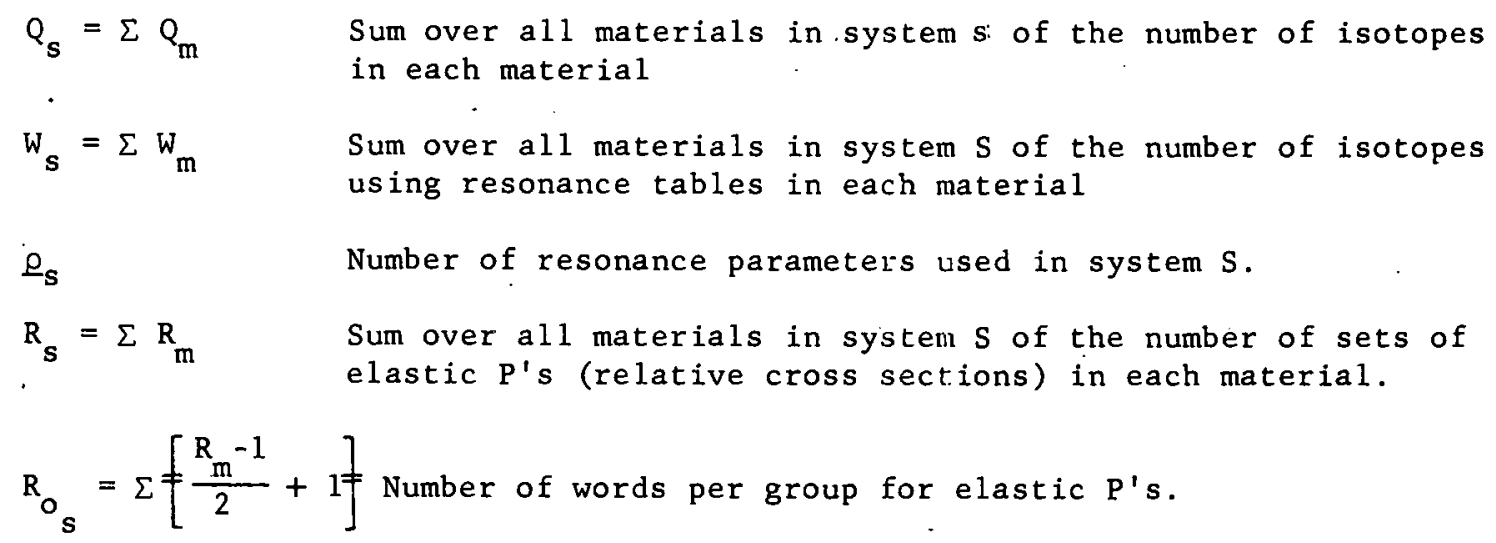


$\mathscr{Z}_{\mathrm{q}_{A}}^{\prime}$

$s^{\prime}$ A

$\varrho_{A}^{A}\left(\begin{array}{c}\rho_{A}-1 \\ =\sum \\ \rho^{\prime}=1\end{array} \varrho^{\prime} \rho^{\prime}\right)$

${ }^{1}$ iA $_{\text {in }}$

$\mathrm{I}_{2 \mathrm{~A}}$

$1_{3 \mathrm{~A}}$

${ }^{\circ} f_{A}$

$I^{\prime \prime} \mathrm{A}$

Total number of atomic weights in atomic weight list for this system previous to these pertaining to this isctope.

Inelastic spectrum table number or level spacing for inelastic spectrum for isotope $A$. If $S^{\prime}{ }_{A}<0,\left|S^{\prime}{ }_{A}\right|=$ spectrum table no.

If $S_{A}^{\prime}>0, S_{A}^{\prime}=1$ level spacing.

$P \Delta V$ table number for isotope $A$.

Total number of entries in resonance parameter tables preceeding resonance parameter table for isotope A.

Lowest group number (highest energy) containing resonances for isotope $A$.

Lowest group number (highest energy) containing resolved resonances for isotope A.

Highest group number (lowest energy) containiug resolved resonances for $A$.

Number of neutrons per fission for resonance range in isotope A.

Highest group number using anisotropic scattering for isotope A.

(any inel) ${ }_{\mathrm{A}}$

(any c) ${ }_{A}$

Statements indicating presence or absence of particular

(any resonances)

(any, $\left.{ }^{f}\right)_{A}$

$B_{\mathrm{A}}$

cross section types in isotope A. Zero if the type does not exist.

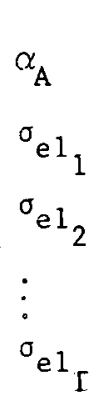

Number of atomic weights for isotope A.

$\left(\frac{a_{1}}{a+1}\right)^{2}$

Elastic scattering cross sections for group $i$, isotope A.

\footnotetext{
$\sigma_{c}$

$\mathrm{o}_{2}$

$\vdots$

$\sigma_{c}$
}

Capture cross sections if (any c) $\neq 0$. 


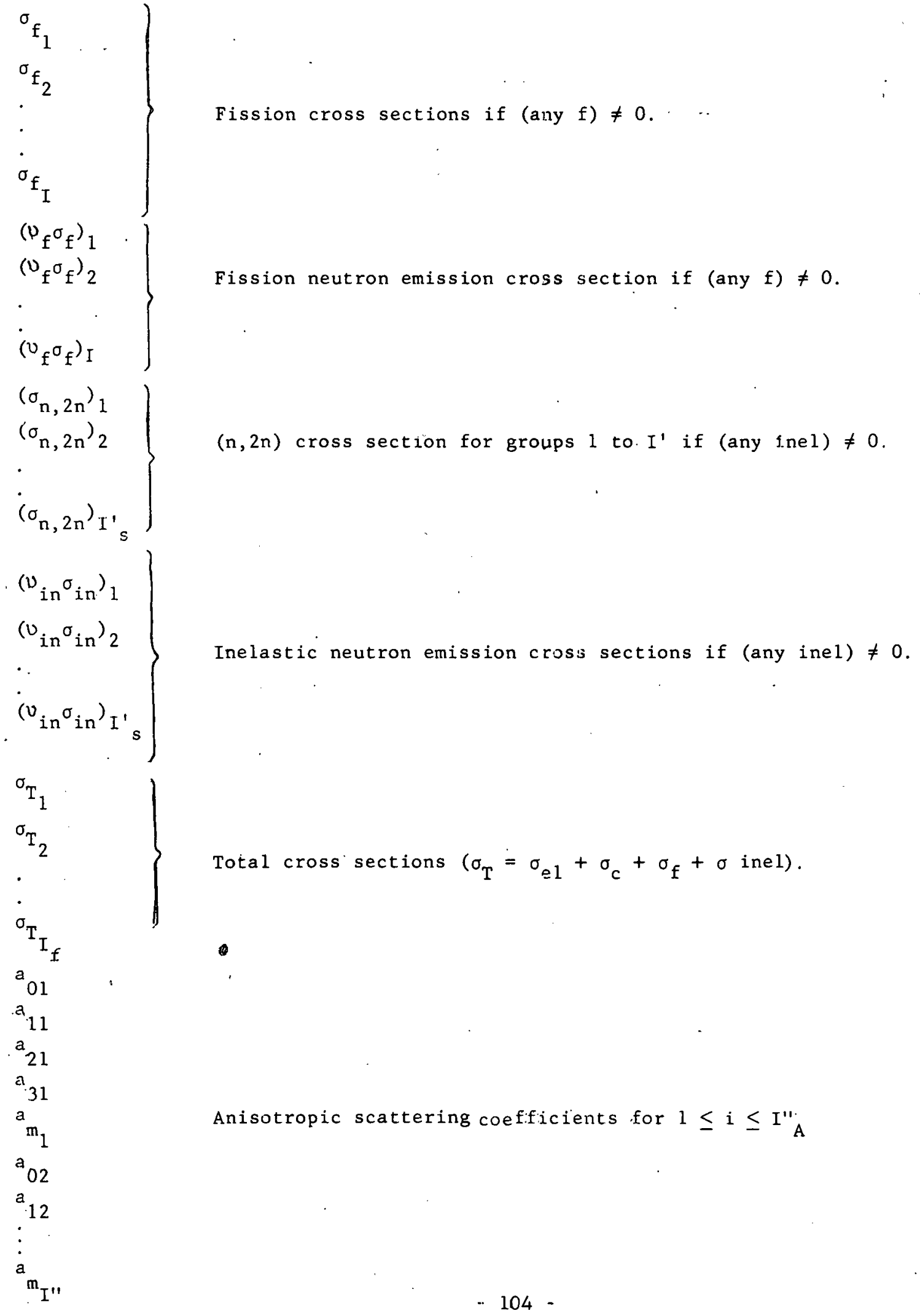


Inelastic scattering coefficient from microscopic group $i^{\prime}$ to macroscopic group $j$ for isotope $A, 1 \leq I^{\prime} \leq I^{\prime} s$ if

(any ine 1$) \neq 0_{j} I_{j}$ is the largest microscopic number in macroscopic group $\mathbf{j}$.

Record $3,4, \ldots, Q_{S}+1$

$\left.\begin{array}{ll}Q_{m}-q_{m} & \\ A & \\ \cdot & \end{array}\right\}$

Repeat for each isotope in system S.

$$
\text { Record } Q_{S}+2
$$

This record contains one record of zeroes for each system on which a monte carlo is never to be done.

$a_{1}$

$a_{I-G_{A}}+1$

$a_{I-G_{A}}+2$

$\dot{a}_{\mathrm{I}}$

First set of atomic weights for first isotope in system s..

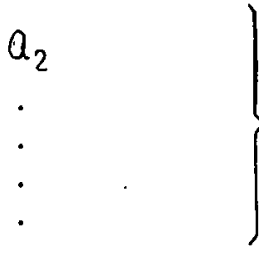

Repeat for each set of atomic weights in system $s$

$\mathrm{I}_{1} \mathrm{I}_{2} \mathrm{I}_{3}$

Resonance group ranges (12 bits each) for first resonance parameter table 


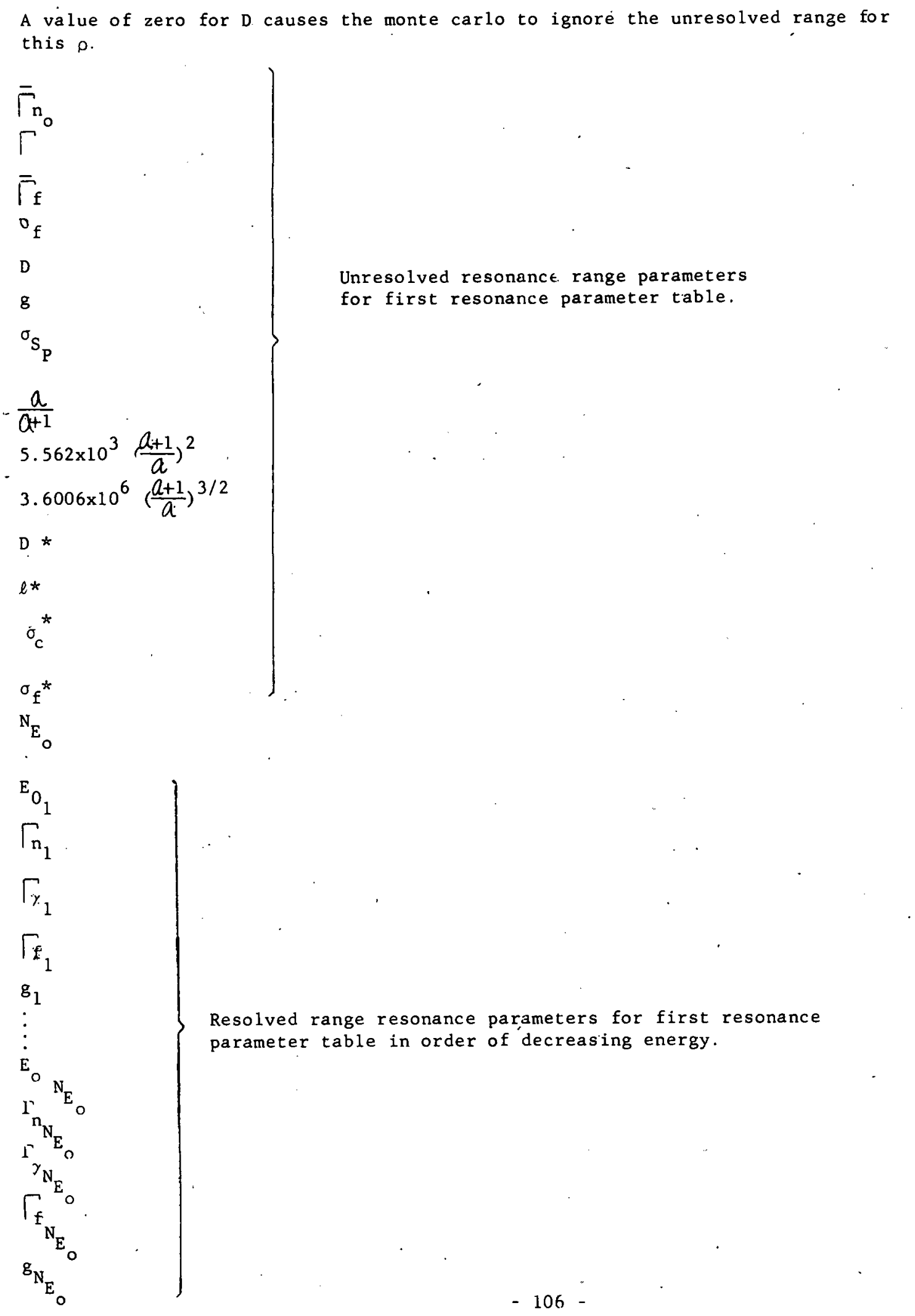




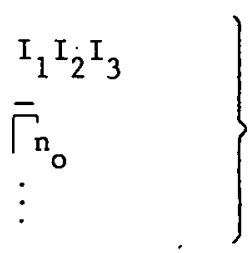

$\left.\begin{array}{l}P(\Delta V)_{1,1} \\ \vdots \\ P(\Delta V)_{17,1}\end{array}\right\}$

$\Delta \mathrm{v}_{1,1}$

:

:

$\Delta \mathrm{V}_{17,}$

$\mathrm{P}(\Delta \mathrm{V})_{1,2}$

:

$\dot{P}(\Delta V)_{17,2}$

$\Delta v_{1,2}$

$P(\Delta V)_{1, \xi_{\mathrm{s}}}$

:

$\Delta V_{17,5 s}$

$P_{1,1}$

$\mathrm{P}_{2,2}$

$:$

$P_{17,2}$

$\left.\begin{array}{l}\mathrm{v}^{2} \text { in }_{1,1} \\ \vdots \\ \mathrm{v}^{2} \text { in }_{17,2 !}\end{array}\right\}$

$\left.\begin{array}{l}\mathrm{P}_{1,2} \\ \vdots \\ \mathrm{P}_{17,2} \\ \mathrm{v}^{2}{ }_{\text {in }}, 2 \\ \vdots \\ \mathrm{P}_{1, \mathrm{~S}^{\prime}} \\ \vdots \\ \mathrm{v}^{2}{ }_{\text {in }}{ }_{17, \mathrm{~S}^{\prime}}\end{array}\right\}$
Repeat for each resonance parameter table used in system $s$.

Probabilities for first $P(\Delta V)$ (velocity increment on new thermal scattering). table, $\mathrm{P}_{1}=1.00, \mathrm{P}_{17}=0$.

Velocity changes for first $P(\Delta V)$ table in order of decreasing velocity.

Repeat for each $P(\Delta V)$ table.

Probabilities for first inelastic spectrum table.

$\mathrm{P}_{1}=1.00, \mathrm{P}_{17}=0.00$

- Energy changes for first inelastic spectrum table in order of decreasing velocity.

Repeat for each spectrum table. 


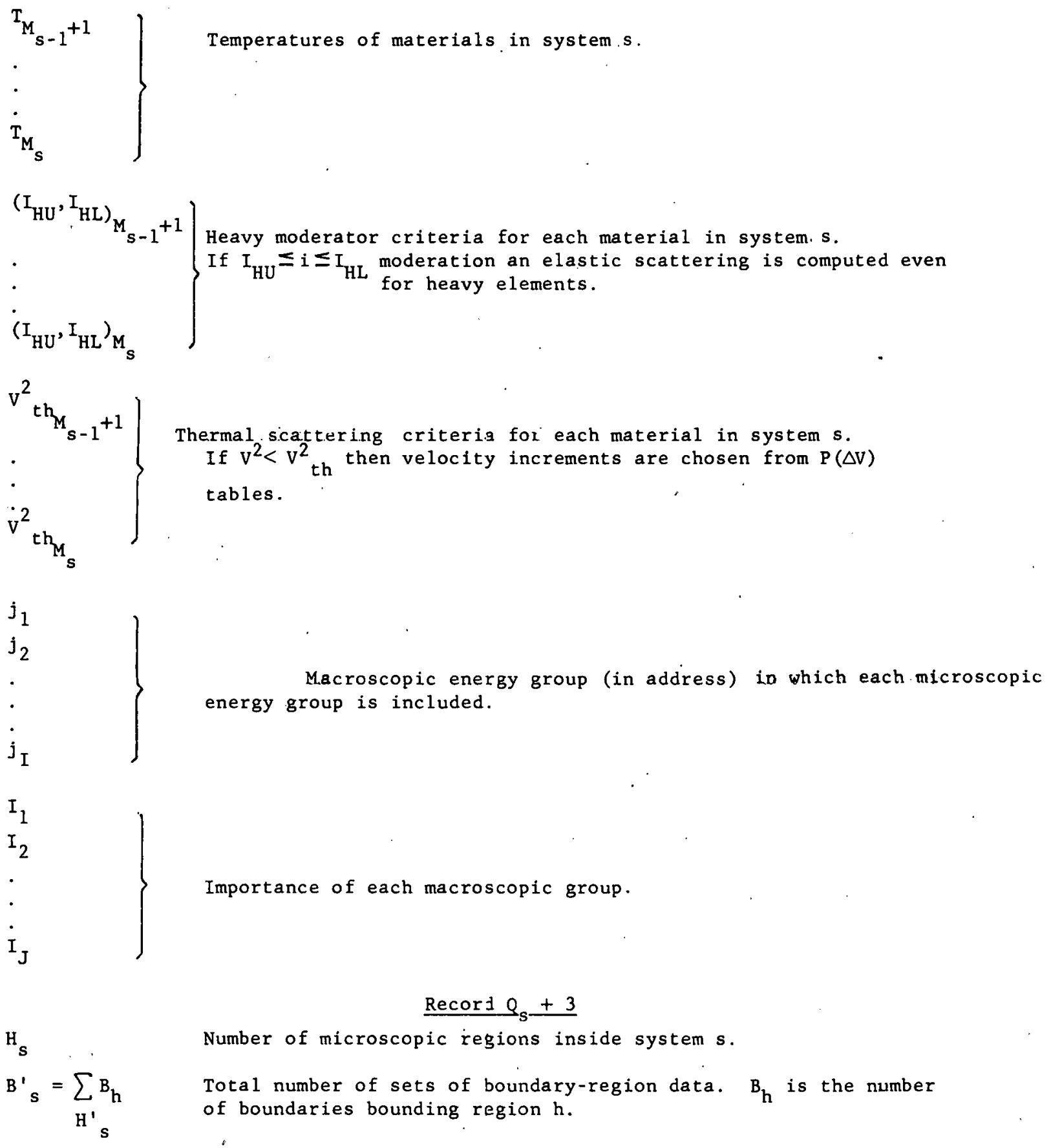

Heavy moderator criteria for each material in system. $s$. If $I_{H U} \leq i \leq I_{H L}$ moderation an elastic scattering is computed even for heavy elements.

$\left(\mathrm{I}_{\mathrm{HU}}, \mathrm{I}_{\mathrm{HL}}\right)_{\mathrm{M}_{\mathrm{S}}}$ $\mathrm{v}^{2}$

Thermal scattering criteria for each material in system $s$ If $V^{2}<V^{2}$ th then velocity increments are chosen from $P(\Delta V)$ tables. energy group is included. 

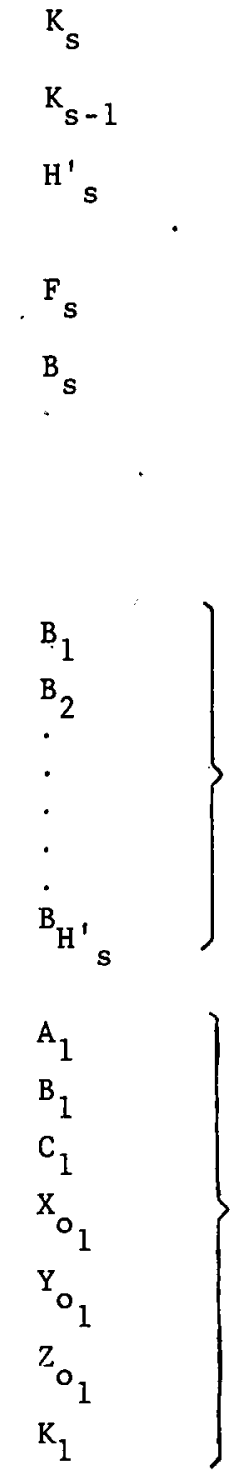

$\mathrm{d}, \mathrm{w}_{\mathrm{b}}$

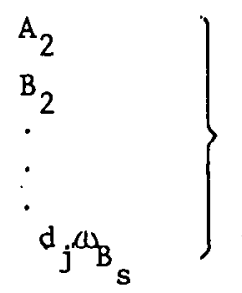

Highest macroscopic region number in system $s$.

Highest macroscopic region number in system $s-1$.

Number of microscopic regions defining system s including outside regions.

Number of region sets in system $s$.

Number of boundaries in system $s$.

$$
\underline{\operatorname{Record} \dot{Q}_{S}+4}
$$

This record contains one word of zeroes, if the system is one on which a monte carlo is never to be done.

Number of boundaries used to describe each region (in address portion of the word only).

Parameters in the boundary equation

$\mathrm{A}\left(\mathrm{X}-\mathrm{S}_{\mathrm{O}}\right)^{2}+\mathrm{B}\left(\mathrm{Y}-\mathrm{Y}_{\mathrm{O}}\right)^{2}+\mathrm{C}\left(\mathrm{Z}-\mathrm{Z}_{\mathrm{o}}\right)^{2}-\mathrm{K}=0$

for first boundary in the system

$\omega_{b}$ in address portion is the index (zero if none) of the set of albedoes to be used with the first boundary; $d$ in decrement portion is the boundary type.

Repeat for each boundary in the system. 


\section{$\underline{\operatorname{Record} Q_{s}+5}$.}

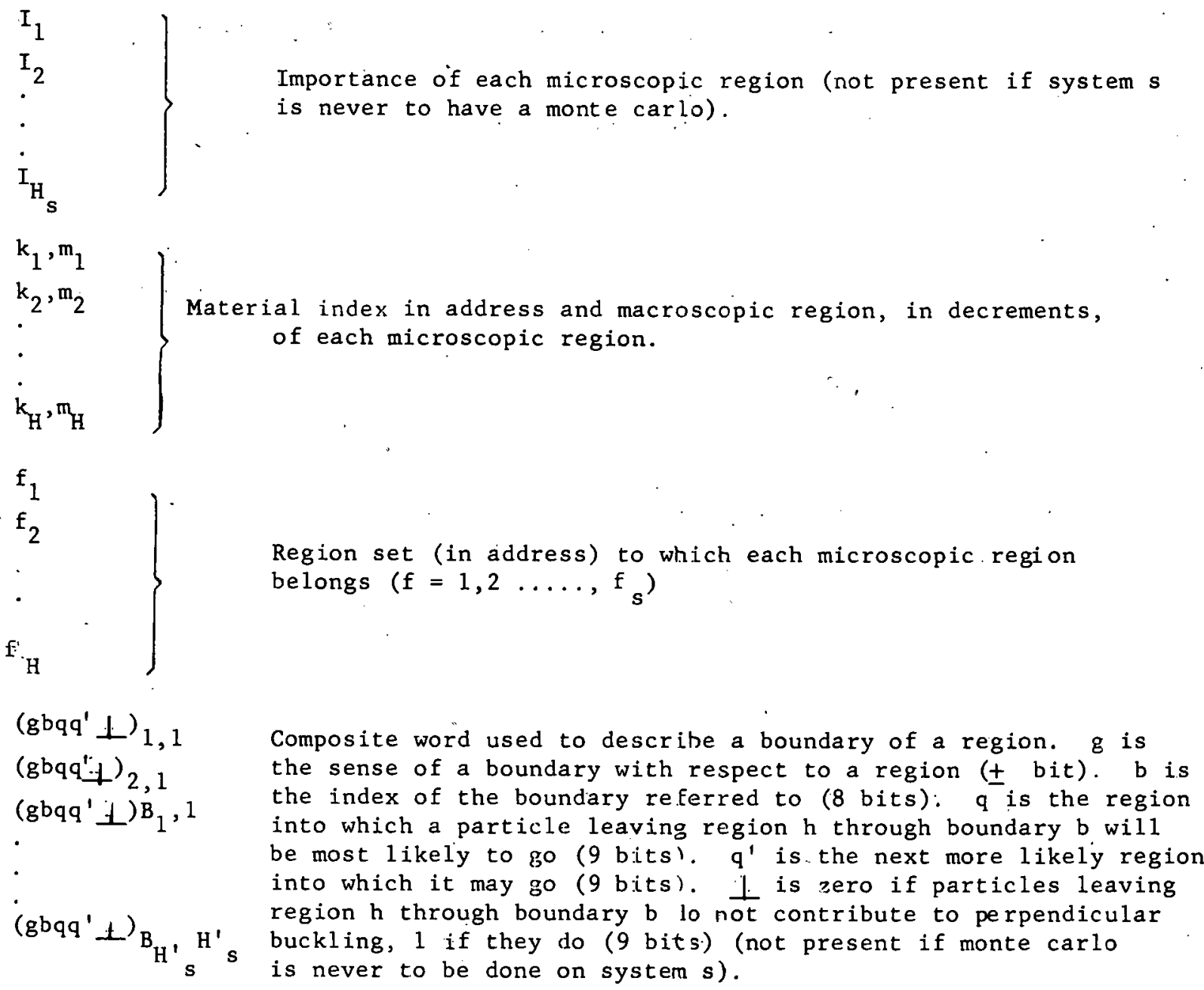

End of File

Repeat for each system. 
F. TAPE 4

Records 1, 3, .., 2m-1, ..,2 $2 \mathrm{M}-1$

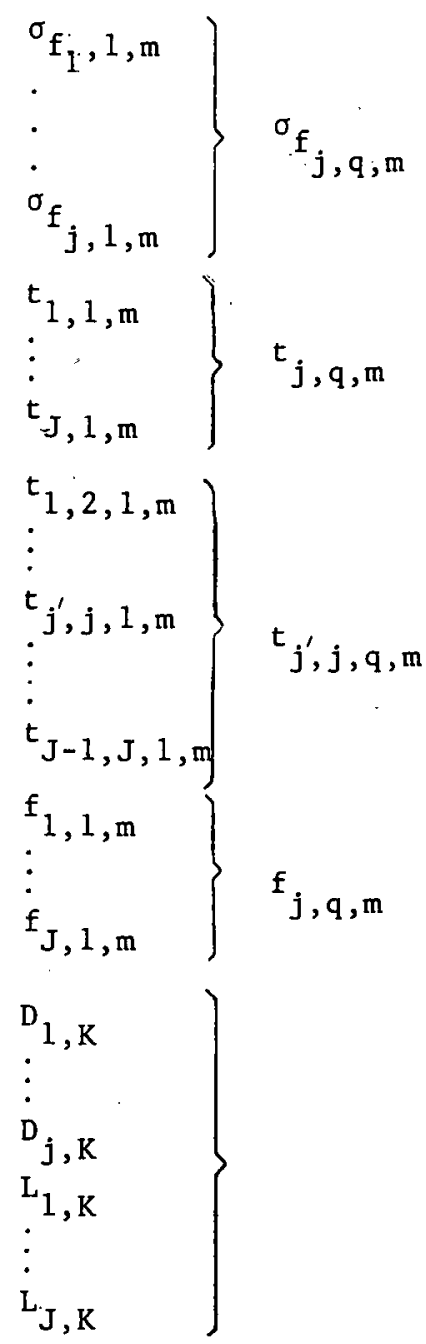

Diffusion calculation cross sections repeat for $\mathrm{q}=1,2, \ldots, \mathrm{Q}_{\mathrm{m}}$

Diffusion coefficients and perpendicular leakage cross sections for macroscopic region. $K$. Present only if $m=M_{K}$.

Records 2, 4, ..,2m, ., , 2M

$\left.\begin{array}{l}\sigma_{o 1,1, m} \\ \sigma_{0, J, 1, m}\end{array}\right\} \begin{aligned} & \text { Capture cross } \\ & \text { sections } \\ & \sigma_{o j, q, m}\end{aligned} \quad$ Not present if (any c) $q, m=0$.

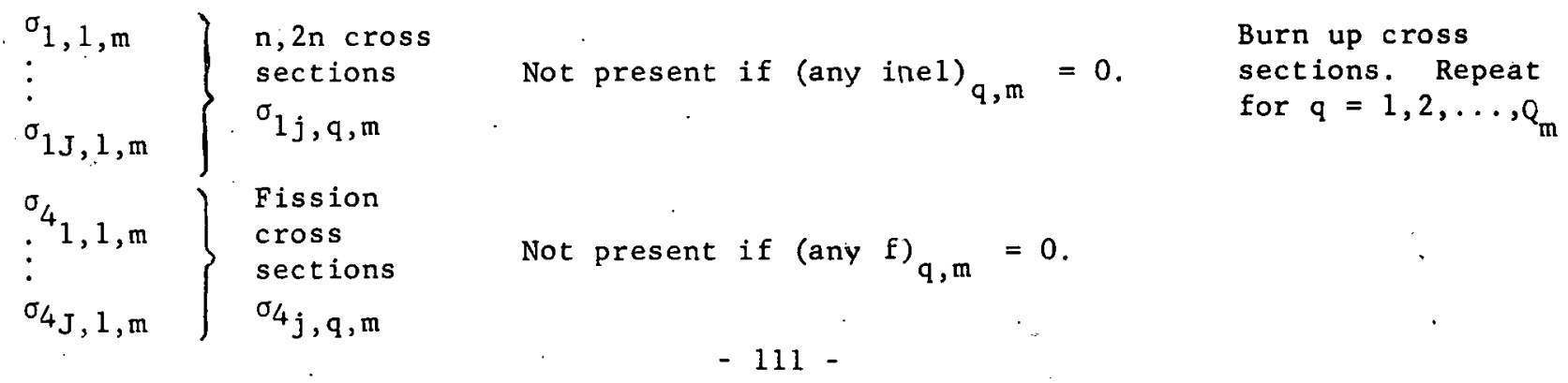


If $\sum_{q=1}^{Q_{m}}[($ any $c)+($ any inel $)+($ any $f)]=0$, replace by one word of zeroes. 
G. TAPE 5 (and 7)

File $1,3,5 \ldots 2 s-1$

Record 1

$\mathrm{s}$

t

$\mathrm{T}$

$T^{\prime}$

$\mathrm{T}_{\mathrm{o}}$

$\mathrm{N}_{\mathrm{MC}}$

$\mathrm{N}_{\text {IV }}$

$\mathrm{X}$

Y

Z

$\alpha$

$\beta$

$\gamma$

$\mathrm{v}$

t

i

h

$\mathrm{S}_{\mathrm{b}}$

(1)

$\alpha$

$\beta$

System number (if $s<0$ region in lex and distance to boundary stored with I.V.'s will be checked. In post monte carlo $s$ is made positive)

Time of previous monte carlo on system s.

Time at which particles reach census. Input routine sets this to $\mathrm{T}_{t}+\mathrm{T}_{o o}$ where $\mathrm{T}_{t}$ is the time interval during which tallies are to be made and $\mathrm{T}_{00}$ is the time during which particles are allowed to come into equilibrium before beginning the tallying in the first time step. After the first time step $T=T_{t}+T_{0}$.

Time to be used is this time step to allow particles to come to equilibrium. Input routine sets this to $\mathrm{T}_{00}$. In all but the first time step it is equal to $\mathrm{T}_{0}$.

Time to be used in all but the first time step to allow particles to come to equilibrium.

Desired number of particles for system s.

Number of I.V. particles for system $S$ on this tape.

$$
\text { Record 2, 3, } 4 \ldots \text { (Ten particles to a record }
$$
plus a possible incomplete record)

(If system $s$ is never to have a monte carlo, the particle coordinates are replaced by one record containing one word of zeroes.)

Particle coordinates, 10 sets to a record.

GBqq' $\perp$ 
File $2,4,6 \ldots 2 \mathrm{~S}$

Record 1

Monte carlo fluxes by microscopic group and region. set. (Initially replaced by one word equal to zero except for those systems on which a monte carlo is never to be done. In those cases, the initial contents of this record are as follows.

(The following data through $\mathcal{L}_{\mathrm{kJ}}$ replaces the flux tallies .. and is provided by the input routine - for systems on which a monte carlo is never to be done)

$\left.\begin{array}{c}\Phi_{11} \\ \Phi_{12} \\ \Phi_{13} \\ \Phi_{\mathrm{fi}} \\ \Phi_{\mathrm{F}_{\mathrm{S}} \mathrm{I}}\end{array}\right\}$

$F_{S} I$ words replacing flux tallies. These are given by $\phi_{f i}=\Psi_{f i} V_{f} \Delta E_{i}$ where the $\psi_{f i}$ are input data*t, $\Delta E_{i}$ is the energy span of group $i$ and $V_{f}$, the relative volume of region set $f$ obtained from $h \sum_{i n} f V_{h}$ **

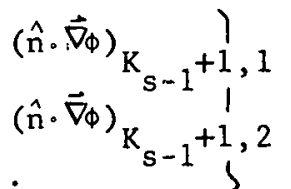
$:$ $\left(\hat{n} \cdot \vec{\nabla}_{\phi}\right)_{k, j}$ $:$ $\left.(\hat{n} \cdot \vec{\nabla} \phi)_{k_{s}, J}\right)$

$\left(\mathrm{K}_{\mathrm{s}}-\mathrm{K}_{\mathrm{s}-1}\right) \mathrm{J}$ words replacing normal gradient tallies. These are given by $\left(\hat{n} \cdot \vec{\nabla}_{\phi}\right)_{k j}=\left[3\right.$ f in k i in j $\left.\dot{A} \sum_{\text {in } m_{f}} N_{A m_{f}} \sigma_{t r_{A i}}{ }_{f i}\right] /$ f $\sum_{\text {in } k \text { i }} \sum_{i=1}{ }^{\phi} \mathrm{fi}$

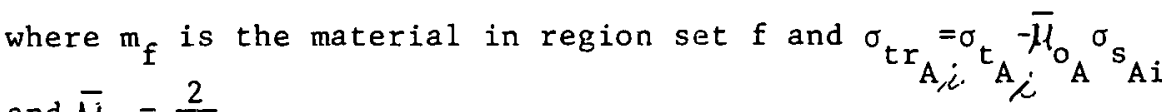
and $\bar{M}_{\mathrm{A}}=\frac{2}{3 \mathrm{~A}}$

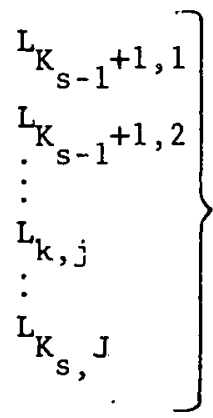

$\left(\mathrm{K}_{\mathrm{S}}-\mathrm{K}_{\mathrm{S}-1}\right) \mathrm{J}$ words replacing leakage tallies each equal to unity. 


$$
\left.\begin{array}{c}
\mathcal{L}_{\mathrm{k}_{\mathrm{s}-1}+1,1} \\
\mathcal{L}_{\mathrm{k}_{\mathrm{s}-1}+1,2} \\
\vdots \\
\mathcal{L}_{\mathrm{k}, \mathrm{j}} \\
\dot{\mathcal{L}} \mathrm{k}, \mathrm{J}
\end{array}\right\}
$$$$
\left(\mathrm{K}_{\mathrm{S}}-\mathrm{K}_{\mathrm{s}-1}\right) \mathrm{J} \text { words replacing perpendicular leakage tallies. }
$$$$
\text { These are given by }
$$$$
\mathcal{L}_{k j}=B_{k}^{2} \frac{1}{\left(n \cdot \nabla_{\phi}\right)_{k j}} \text { where the } B_{k}^{2} \text { are input data. ** }
$$

*The $v_{h}$ are available only for systems on which monte carlo's are never to be done. For conventence, they are found in the input in the locations normally occupied by the $I_{h}$.

**The $\mathrm{B}_{k}{ }^{2}$ and ${ }^{t} f i$ are special input data required only for systems on which a monte carlo is never done. $B^{2}{ }_{k}$ is normally something like $\frac{\pi^{2}}{\mathrm{H}^{2}}$ and $\psi_{f i}$ is a relative flux density.

$$
\text { Records 2, 4,6 ..2 }\left(M_{s}-M_{s-1}\right)
$$

Each record a material (initially each of these records contains one word equal to zero).

$$
\left.\begin{array}{l}
\sigma_{f_{1,1, m}} \\
\sigma_{f_{2,1, m}} \\
\sigma_{f_{J, 1, m}} \\
t_{1,1, m} \\
t_{J, 1, m} \\
t_{1,2,1, m} \\
t_{1,3,1, m} \\
t_{2,3,1, m} \\
\vdots \\
t_{j}{ }^{\prime}, j, 1, m \\
\vdots \\
\vdots \\
t_{J-1, J, 1, m} \\
f_{1,1} \\
\vdots \\
f_{J, 1} .
\end{array}\right\}
$$

Effective microscopic cross sections computed in post monte carlo for pre-diffusion, as indicated in equation (100), (101), and (103), these are not, strictly speaking, cross sections, since they have been normalized by $\Sigma \phi_{i m}$ instead of simply by $\phi_{\text {im }}$.

Repeat for $q=2, \ldots, Q_{m}$. 


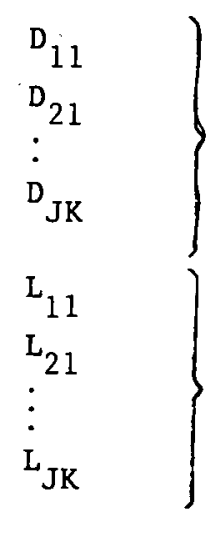

Diffusion coefficients. This section appears only for materials $\mathrm{m}=\mathrm{M}_{\mathrm{K}}$.

Perpendicular leakage cross sections. This section appears

only for materials $\mathrm{m}=\mathrm{M}_{\mathrm{K}}$

$\left.\underline{\text { Records } 3,5 \ldots 2\left(M_{S}-M\right.}{ }_{S-1}\right)+1$

$\left.\begin{array}{l}\sigma_{0_{1,1, m}} \\ \sigma_{0}{ }^{\sigma_{2,1, m}} \\ \sigma_{0} \\ \sigma_{\mathrm{J}, 1, \mathrm{~m}} \\ \vdots \\ \sigma_{1,1, \mathrm{~m}} \\ \sigma_{\mathrm{J}}{ }_{1,1, \mathrm{~m}} \\ \vdots \\ \sigma_{4} \\ \sigma_{\mathrm{J}, 1, \mathrm{~m}}\end{array}\right\}$

Each material a record, initially each of these records contains a word of zeroes.

Effective'microscopic cross sections computed in the post monte carlo for the pre-burnup for decomposition of isotope $q_{m}$. by mode $m$ by neutrons in group $j$. Modes in this series are 0 for capture (present only if (any $c) \neq 0 ; 1$ for $(n, 2 n$ ) (present only if (any inel) $\neq 0$, and 4 for all fission (present only if (any $f$ ) $\neq 0$. (Repeat for $q=2, \ldots, Q_{m}$ ).

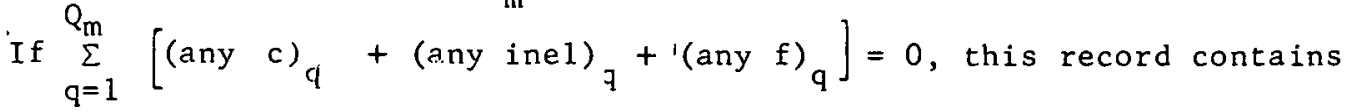
ane word of zeroes. 
H. TAPE 6

File 1

Record 1, 2, 3...M (each material a record)

(For a material on which a burnup is never done the record is simply one word equal to zero)

m

$\Sigma \mathrm{D} \quad$ Number of direct descendants in material $\mathrm{m}$

$q=1$

$\mathrm{D}_{1 \mathrm{~m}}$
$\mathrm{D}_{2}{ }_{\mathrm{m}}$
$\dot{\mathrm{D}}_{\mathrm{Q}_{\mathrm{m}}}$

\}

$\left(q_{1}, 1 ;\right.$ mode $)$

Direct descendant number (q) and mode of production ( $q$ in

decrement, mode in address) for isotope 1 in order of increasing

$\left(\mathrm{q}_{2,1} ;\right.$ mode $)$

mode number including only those required. Mode code: 0 = capture;

$\left(\mathrm{q}_{3,1} ;\right.$ mode $)$

$1=(n, 2 n) ; 2$ = decay with first decay constant; 3 = decay with second

decay constant; 4 through 9 = fission with respective fission yields

(only the descendants for fission modes up to but not including the

(q mode) first for which $y=0$ are present).

.

$\mathrm{q}_{\mathrm{d}, \mathrm{q}_{\mathrm{m}}^{\prime}} \quad\left(\mathrm{d}=1,2,3, \ldots, \mathrm{D}_{\mathrm{q}^{\prime}{ }_{\mathrm{m}}{ }^{\prime}}\right.$

$\left(\mathrm{q}_{\mathrm{D}_{\mathrm{m}}}, 1 ;{ }^{\text {mode }}\right)$

$\left(\mathrm{q}_{1,2} ;\right.$ mode $)$

$\left(\mathrm{q}_{2,2 ; \text { mode })}\right.$

Repeat for each isotope in material $\mathrm{m}$

-

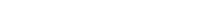

$y_{1,1}$

$\mathrm{y}_{2,1}$

$\mathrm{y}_{3,1}$

.

-

$y_{6,1}$

Fission yields for six descendants by fission of first isotope 
$\left.\begin{array}{l}y_{1,2} \\ y_{2,2} \\ \vdots\end{array}\right\}$

Repeat for each isotope in material $\mathrm{m}$

$\lambda_{1,1}$

Decay constant for mode 2, isotope 1 .

$\lambda_{2,1}$

Decay constant for mode 3 , isotope 1 .

$\left.\begin{array}{l}\lambda_{1, q}, 1 \\ \lambda_{2, q} \\ \lambda_{2, Q_{m}}\end{array}\right\}$

Repeat for each isotope in $m$

$\left.\begin{array}{cc}\sum_{\mathrm{q}=1}^{\mathrm{Q}_{\mathrm{m}}} & \\ \mathrm{D}_{1} & \\ \vdots & \mathrm{D}_{\mathrm{m}} \\ \vdots & \end{array}\right\}$

Repeat for each material (each material a record)

File 2

Record 1

$t$

$\Delta t$

$t_{\text {max }}$

M

$\mathrm{K}$

$\mathrm{J}$

$\sum_{m} Q_{m}$

$\epsilon_{1}$

$\epsilon_{2}$

time (initially zero)

Basic time step (days)

Time at which problem is to end (days)

Number of materials

Number of macroscopic regions

Number of macroscopic groups

Total number of isotopes

Tolerance limit for increase of tine step

Tolerance limit for decrease of time step

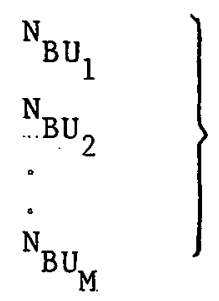

Number of diffusion steps between burnup calculations on each material 


$$
\left.\begin{array}{l}
\pi_{1} \\
\pi_{2} \\
\pi_{M}
\end{array}\right\}
$$

Diffusion steps since last burnup on each material. For materials not to be burned up (including all control materials), $\pi_{M}=1$, $\mathrm{N}_{\mathrm{BU}_{\mathrm{m}}}=32767$ (for those for which burnup calculations are to take* place $\pi_{m}=N_{B U_{m}}$ initially)

Number of backward differences of rates of change of concentration available for predictions on each material. Initially zero.

Number of isotopes in each material.

$\left.\begin{array}{l}M_{1} \\ M_{2} \\ \dot{M}_{K}\end{array}\right\}$

Highest material number in each macroscopic region.

\section{Record 2}

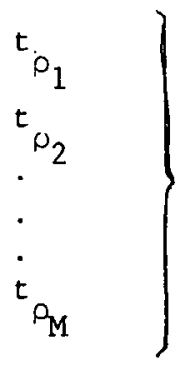

Time for which last concentration predictions on material $\mathrm{m}$ were made. Initially zero.

Latest corrected concentrations for each isotope of each material. These, like the predictors following, are initially equal to the input values. 


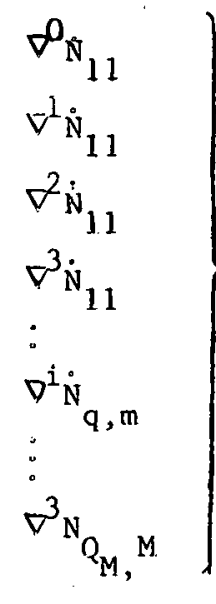

Backward differences for each isotope of each material (initially $\underset{m=1}{4 \pi} \bigcap_{m}^{M}$ words all ::ero)

\section{Record 3}

$t$

time

$\Delta$ t.

Basic time step

$\left.\begin{array}{c}\pi^{\prime} 1 \\ \pi^{\prime} 2 \\ \vdots \\ \pi_{M}^{\prime}\end{array}\right\}$

$\pi_{M}^{\prime}=0$ if a burnup was not done on material $\mathrm{m}$ in previous time step; 1 if it was.

\section{Record 4,5,...M +3}

$\left.\begin{array}{ll}p & N_{1} \\ p & N_{2} \\ : & \\ p & N_{Q_{m}}\end{array}\right\}$

Latest predicted concentrations for material m (initially equal to the input values) 
I. TAPE 8

\section{Record 1}

$\Phi_{11}$
$\Phi_{21}$
$\cdot$
$\cdot$
$\Phi_{\mathrm{J}}$
$\dot{\Phi_{\mathrm{JK}}}$

Diffusion Fluxes

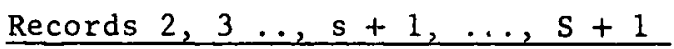

$\left.\begin{array}{c}\omega_{1} \\ \omega_{2} \\ \cdot \\ \omega_{\mathrm{J}}\end{array}\right\}$

Albedoes* for the inner (nearest the origin) boundary of system $s$.

Albedoes for the outer boundary if system $s$.

*Although albedoes are computed by the post-diffusion for the inner and outer surfaces of each system, the monte carlo only uses those indicated by the boundary data. 
I. TAPE 9

\section{Record 1}

$S$

M

$\mathrm{J}$

$\frac{1}{2} \mathrm{~J}(\mathrm{~J}-1)$

p

$\epsilon_{3}$

$\epsilon_{4}$

$\mathrm{J*}, 0$

W

$\mathcal{L}$

${ }^{\mathrm{B}}{ }_{\mathrm{w}}$

$\mathrm{V}$

$\mathrm{N}$

$\mathrm{K}$

R

$\left.\begin{array}{l}\Delta \dot{r}_{1} \\ \Delta r_{2} \\ \dot{ }_{2} \\ r_{K}\end{array}\right\}$

$\mathrm{B}_{01}$

$\mathrm{B}_{02}$

B

Number of monte carlo systems.

Number of materials

Number of macroscopic groups

Index giving the geometry of the diffusion calculation;

0 means slab, 1 cylindrical, 2 spherical.

Tolerance for convergence of effective multiplication

Tolerance for convergence of neutron source distribution.

Two's complement of number of macroscopic groups in decrement.

Acceleration factor.

Number of control materials.

Number of sets of albedoes (this is always equal to $2 \mathrm{~S}$ although the monte carlo calculation may not use them all.

$\sum_{n=0}^{N} \quad r_{n}^{\rho}\left(\frac{\Delta r}{2}+\frac{\Delta r_{n+}}{2}\right)$

Highest mesh point index.

Number of macroscopic regions:

Value of the coordinate in the fiffusion calculation at the left most (Zeroth) mesh point (normally zero).

Mesh spacing in macroscopic region $\mathrm{k}$.

Boundary condition parameters a : left end of mesh. 


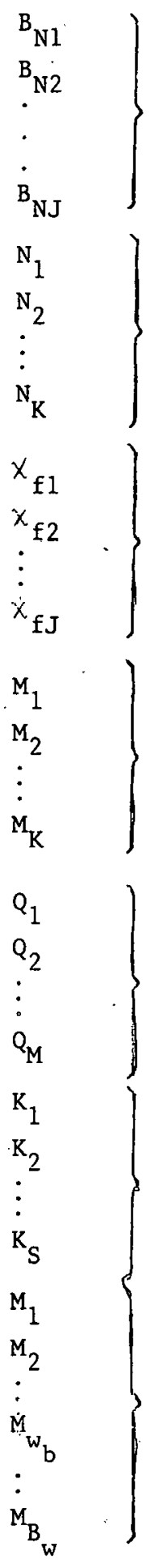

Boundary condition parameters at right endoof mesh.

Highest mesh point number in macroscopic region $\mathrm{k}$.

Fraction of fission neutron emerging from fission in group j per unit energy.

Highest material number in each nacroscopic region.

Number of isotopes in each material

Highest macroscopic region number (rightmost region) in each system. (Used as left region, in each pair of albedoes).

Correction factor used in computing the $w_{b}^{\text {th }}$ set of albedoes. 
Record 2

$\left.\begin{array}{c}\epsilon_{5} \\ \mathrm{M}_{1} \\ \mathrm{M}_{2} \\ \mathrm{M}_{\ell} \\ \vdots \\ \mathrm{M}_{\chi}\end{array}\right\}$

Material indices corresponding to control material indices 1 through $\mathcal{L}$

Concentrations of isotopes for each value of $c$ for each control material $(c=1,2)$

Record $3,4 \ldots K+2$

Each macroscopic region $a$ record.

$\mathrm{D}_{1 \mathrm{k}}$

$\mathrm{D}_{2 \mathrm{k}}$

:

$\mathrm{D}_{\mathrm{Jk}}$

$\mathrm{T}_{1 \mathrm{k}}$

$\mathrm{T}_{2 \mathrm{k}}$

:

$\mathrm{T}_{\mathrm{Jk}}$

$\mathrm{T}_{12 \mathrm{~K}}$

$\mathrm{T}_{13 \mathrm{k}}$

$\mathrm{T}_{23 \mathrm{k}}$

$\mathrm{T}_{1.4 \mathrm{k}}$

$\mathrm{T}_{24 \mathrm{k}}$

$\mathrm{T}_{34 \mathrm{k}}$

:

$\mathrm{T}_{\mathrm{j}}{ }_{\mathrm{jk}}$

:

$\mathrm{T}_{\mathrm{j}-1, \mathrm{~J}, \mathrm{k}}$

Diffusion cross sections. Initially zeroes. 


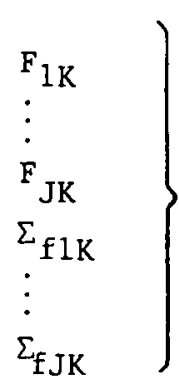

Diffusion cross sections.

$\underline{\operatorname{Record} K+3}$

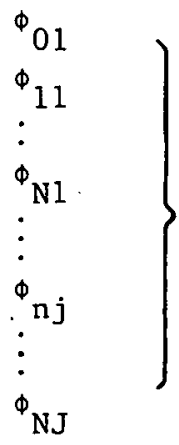

Diffusion fluxes per unit energy in each group at each mesh point. Initially zeroes.

$\underline{\operatorname{Record} \mathrm{K}+4}$

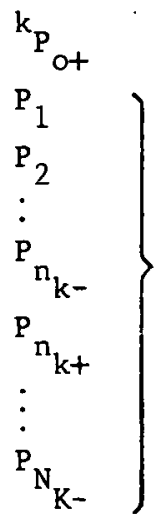

Effective multiplication and neutron source distribution. Initially, $\mathrm{B}_{\mathrm{n}}$ and $\mathrm{k}=1.0$.

\section{Record $K+5$}

Control index of active control region set

$\mathrm{P}_{\mathrm{a}}$

$P$ for above $\left(P=\frac{\mathrm{p}_{\mathrm{q}, \mathrm{m}_{\ell}}-\mathrm{N}_{\mathrm{q}, 1, \ell}}{\mathrm{N}_{\mathrm{q}, 2, \ell}-\mathrm{N}_{\mathrm{q}, 1, \ell}}\right)$

$c_{1}$
$c_{2}$
$\vdots$
$c$

$\frac{1}{c_{2}}=$ latest known value of $\frac{\mathrm{dk}}{\mathrm{dP}} \ell$

(Initially values specified in the input. 
K. RBU OUTPUT TAPE, TAPE 10

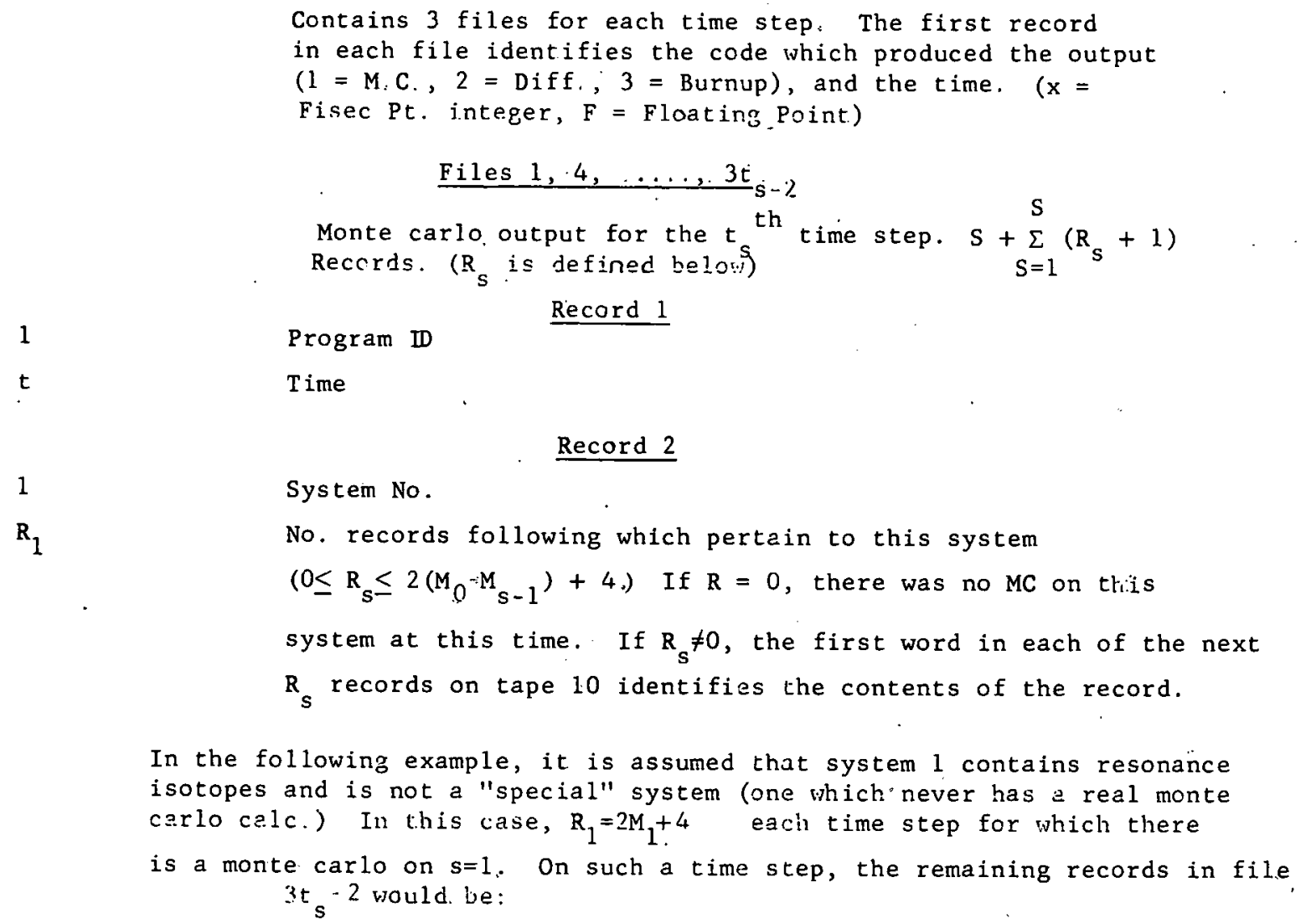

In the following example, it is assumed that system 1 contains resonance isotopes and is not a "special" system (one which"never has a real monte carlo calc.) In this case, $R_{1}=2 M_{1}+4$ each time step for which there

is a monte carlo on $s=1$. On such a time step, the remaining records in file $3 t_{s}-2$ would. be:

\section{Record 3 :}

1

N

1.0

N

$N / I_{j} I_{h}$ 1.0

N

$\mathrm{N} / \mathrm{I}_{\mathrm{j}} \mathrm{I}_{\mathrm{h}}$

$\pi\left(1-\mathrm{P}_{2}\right)$

$1 / I_{j} I_{h}$

$\pi\left(1-\mathrm{P}_{2}\right) \mathrm{P}_{\mathrm{T}}$

$N \perp / I_{j} I_{h}$

$P_{T} / I_{j} I_{h}$
ID code for Code Check Quantities

Particles Rejected

Initial coordinates

Initial Particles

Initial neutrons

Coordinates reaching census

Particles reaching census

Neutrons reaching census

Neutrons, expected, to collision.

Neutrons to collision

N. utrons, expected, from collision

Neutrons from collision

Neutrons, expected from collision 


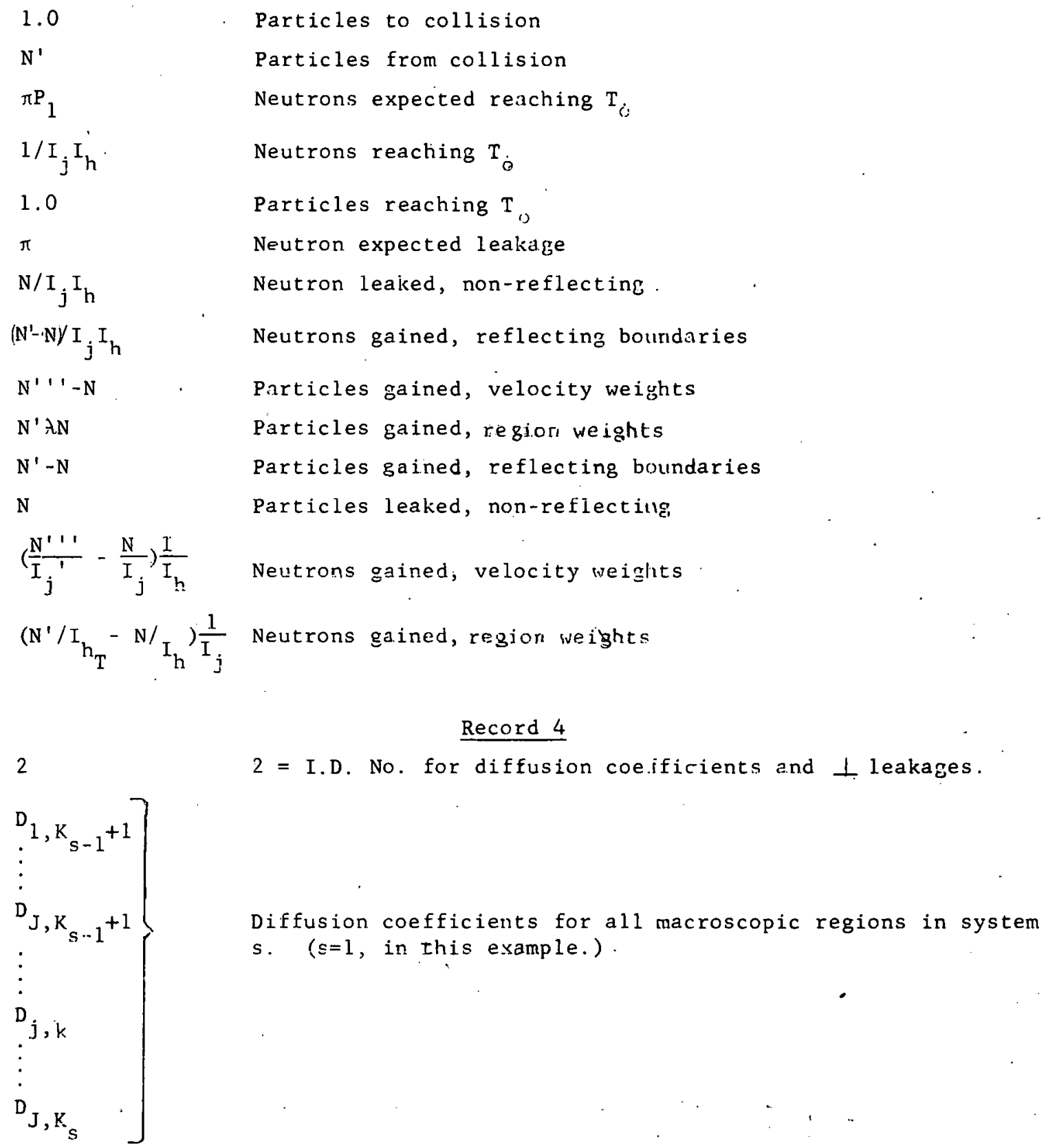




$$
\left.\begin{array}{l}
\left(B^{2} D\right)_{1, K_{s-1}}+1 \\
\vdots \\
\left(B^{2} D\right)_{J, K_{S-1}}+1 \\
\vdots \\
\left(B^{2} D\right)_{j, k} \\
\vdots \\
\left(B^{2} D\right)_{J, K_{S}}
\end{array}\right\}
$$

Perpendicular leakages for all macroscopic regions in system 1 .

\section{Record $5,6 \ldots 4+M_{1}$}

3

m

$$
\left.\begin{array}{l}
\sigma_{f_{1,1, m}} \\
\sigma_{f_{j, 1, m}} \\
\vdots \\
\sigma_{f_{J, 1, m}}
\end{array}\right\}
$$$$
\left.\begin{array}{l}
\mathrm{t}_{1,2,1, \mathrm{~m}} \\
\mathrm{t}_{1, j, 1, \mathrm{~m}} \\
\mathrm{t}_{1, J, 1, m} \\
\mathrm{t}_{2,2,1, \mathrm{~m}} \\
\vdots \\
\mathrm{t}_{\mathrm{j}^{\prime}, \mathrm{j}, 1, \mathrm{~m}} \\
\mathrm{t}_{\mathrm{J}-1, \mathrm{~J}, 1, \mathrm{~m}}
\end{array}\right\}
$$

I.D. code for microscopic diffusion constants Material index of the material to which the following data apply.

Fission cross sections* for first isotope of material m

Total cross sections* for first isotope of material $\mathrm{m}$
Transfer cross sections* from group $j^{\prime}$ to $j$ for first isotope of material m 


$$
\left.\begin{array}{l}
\mathrm{f}_{1,1, \mathrm{~m}} \\
\mathrm{f}_{\mathrm{j}, 1, \mathrm{~m}} \\
\mathrm{f}_{\mathrm{J}, 1, \mathrm{~m}}
\end{array}\right\}
$$$$
\text { Fission neutron production* cross sections for first isotope of }
$$$$
\text { material m }
$$

Microscopic diffusion cross sections* (for macroscopic groups) as above for second isotope in material $m$ of system $s$.

Diffusion cross sections for remaining isotopes of material m

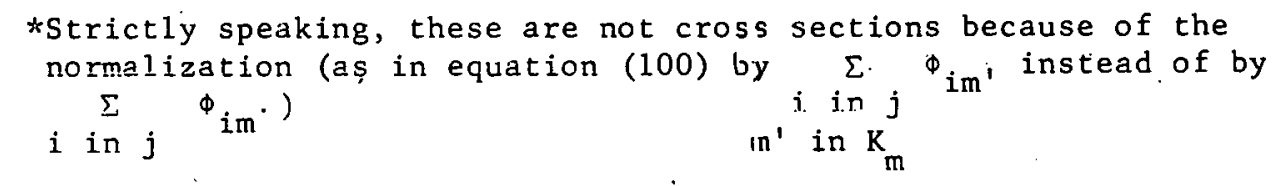

\section{$\underline{\text { Record } 6,8,10 \ldots 4+2 M_{1}}$}

4

m

$$
\left.\begin{array}{l}
\sigma_{\mathrm{o}, 1, \mathrm{~m}} \\
\sigma_{\mathrm{o}, \mathrm{J}, 1, \mathrm{~m}} \\
\sigma_{1,1,1, \mathrm{~m}} \\
\vdots \\
\sigma_{1, \mathrm{~J}, 1, \mathrm{~m}} \\
\sigma_{4,1,1, \mathrm{~m}} \\
\vdots \\
\sigma_{4, \mathrm{~J}, 1, \mathrm{~m}} \\
\sigma_{1,1,2, \mathrm{~m}} \\
\vdots \\
\sigma_{\mathrm{m}}, \mathrm{j}, \mathrm{q}, \mathrm{m} \\
\vdots \\
\sigma_{4, \mathrm{~J}, \mathrm{Q}},{ }^{\prime}
\end{array}\right]
$$

I.D. code for microscopic burnup constants.

Material index of the material to which the following data apply.

Effective microscopic cross sections* for burnup by decomposition of isotope $\mathrm{q}$ by mode $\mathrm{m}$ by neutrons in group $j$. Modes in this series are 0 for capture (present only if (any $c) \neq 0$ ); 1 for $(n, 2 n)$ (present only if (any inel) $\neq 0$ ); and 4 for fission (present only if (any $f_{-}$) $\neq 0$.

* Refer to note in Record $5,6 \ldots 4+M_{1}$, above. 


\section{$\underline{\text { Record } 5+M}$}

4

$\widetilde{\sigma}_{s, J U}, 1$

$\widetilde{\sigma}_{\mathrm{c}, \mathrm{JU}}, 1$

$\tilde{\sigma}_{f, J U}, 1$

$\tilde{v}_{s, J U_{s}}+1,1$

$\tilde{\sigma}_{c, J U_{s}}+1,1$

$\tilde{\sigma}_{f, U_{s}}+1,1$

:

$\tilde{\sigma}$

$\tilde{\sigma}_{\mathrm{s}, \mathrm{JL}}, 1$

$\tilde{\sigma}_{\mathrm{C}, \mathrm{JL}} \mathrm{s}$,

$\tilde{\sigma}_{f, J L}, 1$

I.D. code for resonance cross sections.

Resonance cross sections for macroscopic group. $\mathrm{JL}_{s}$, for

the first resonance isotope in syztem $s$.

$$
\begin{aligned}
& \vec{\sigma}_{r, j, w}=\left(\Sigma_{r}\right)_{w, j, m} / \sum_{j}{ }_{i . m}{ }^{N}, w \\
& \text { lor. wis } \\
& \text { w has resonafice.: }
\end{aligned}
$$

Repeat above for rescnance isotcpes $2,3, \ldots \ldots, w^{\prime}$,

\section{Record $6+2 M_{1}$}

I.D. No. for M.C. fluxes

Normalized monte carlo fluxes for sustem $s$.

$$
\underline{\operatorname{Record} s+\sum_{:=1}^{s-1} R+1}
$$

s

$\mathbf{R}_{\mathbf{S}}$

:

etc.

: 


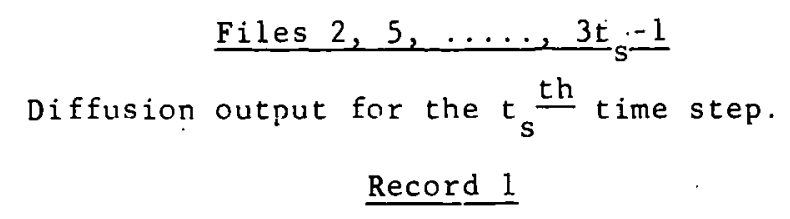

Program I.D.

Time

\section{$\underline{\text { Record } 2}$}

k

$l_{\mathrm{a}}$

$\mathrm{P}_{\mathrm{l}}$

$c_{\ell_{a}}$

Record 3

$\left.\begin{array}{l}\Phi_{1,0} \\ \vdots \\ \Phi_{j}, 0 \\ \vdots \\ \Phi_{\mathrm{J}, 0} \\ \vdots \\ \Phi_{\mathrm{j}, \mathrm{n}} \\ \Phi_{\mathrm{J}, \mathrm{N}}\end{array}\right]$

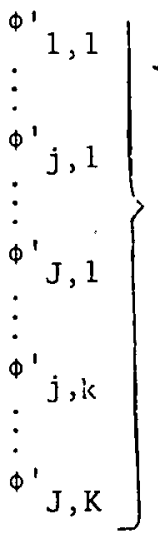

Diffusion fluxes, by macroscopic group and mesh point.

$\Phi_{j, n}$, averaged over each region.

\section{Record 4}

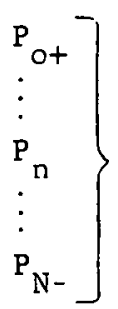

Power, by mesh point 
$\left.\begin{array}{l}\mathrm{P}_{1} \\ \vdots \\ \mathrm{P}_{\mathrm{k}} \\ \vdots \\ \mathrm{P}_{\mathrm{K}}\end{array}\right\}$

Power, by region

Record 5

$\left.\begin{array}{l}\omega_{1,1} \\ \omega_{2,1} \\ \vdots \\ \omega_{\mathrm{j}, 1} \\ \vdots \\ \omega_{\mathrm{J}, 1} \\ \omega_{1,2} \\ \vdots \\ \omega_{\mathrm{J}, 2} \\ \vdots \\ \omega_{\mathrm{j}, ~(\omega)} \\ \vdots \\ \omega_{\mathrm{J}, \mathrm{B}_{(\mathrm{U}}}\end{array}\right\}$

Albedoes, $2 \times \mathrm{JxS}$ words.

$\underline{\text { Record } 6}$

$\left.\begin{array}{l}\mathrm{T}_{1,1} \\ \mathrm{~T}_{2,1} \\ \vdots \\ \mathrm{T}_{\mathrm{j}, 1} \\ \vdots \\ \mathrm{T}_{\mathrm{J}, 1} \\ \vdots \\ \mathrm{T}_{\mathrm{j}, \mathrm{k}} \\ \vdots \\ \mathrm{T}_{\mathrm{J}, \mathrm{K}}\end{array}\right\}$

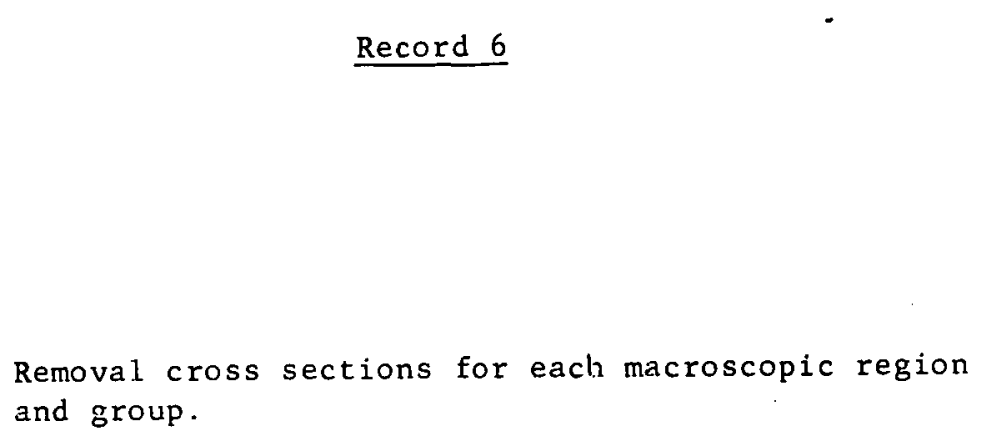
and group. 


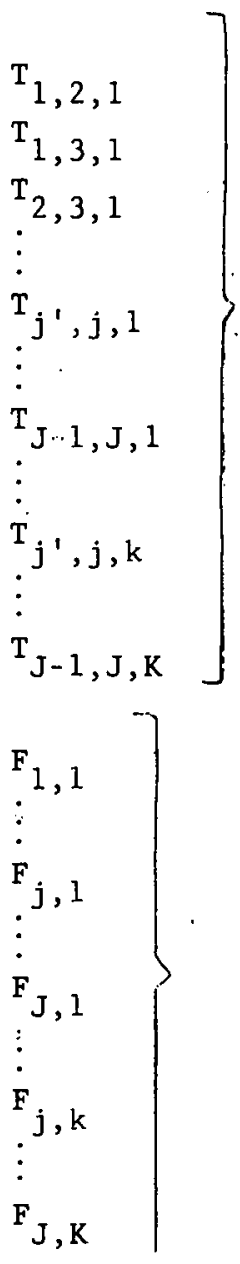

Transfer coefficients.

Fission neutron production cross sections.

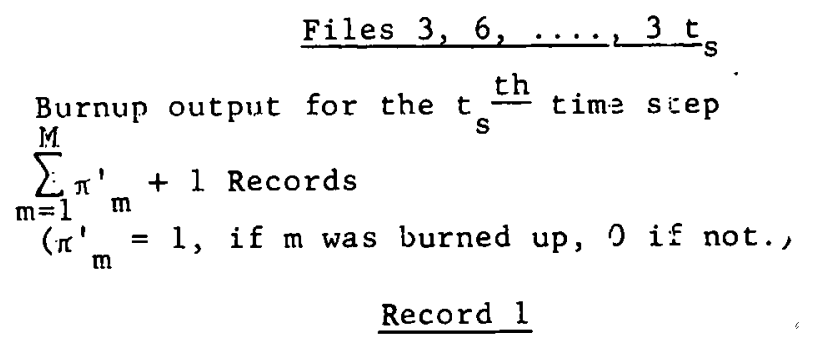

3

Code I.D.

Time

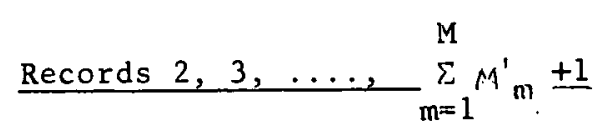

m

Material index 


$$
\left.\begin{array}{l}
\mathrm{N}_{1, m} \\
\mathrm{~N}_{2, m} \\
\vdots \\
\mathrm{N}_{\mathrm{q}, \mathrm{m}} \\
\vdots \\
\mathrm{N}_{\mathrm{q}_{\mathrm{m}}, \mathrm{m}}
\end{array}\right\}
$$

-

Latest set of corrected isotopic concentrations in material $\mathrm{m}$. 
A. Diffusion Code

(Number of words in block.)

I. RBU monitor

II. Coding - Diffusion.

1. Pre-diffusion

2. Diffusion

3. Iteration on reactivity

4. Post diffusion

III. Coding - Subroutines.

1. Redundancy check routine

2. $r$ exp. $\rho$ routine

3. Tape dispatcher

IV. Permanent diffusion data.

1. Constants

2. $\mathrm{BCI}$

3. DSC commands

v. "Common" storage.

61 words - input data

VI. "Eraseable" storage.

63 words - intermediate results, indirect address block (LWA is "ADRR")

Block VII is variable length storage:

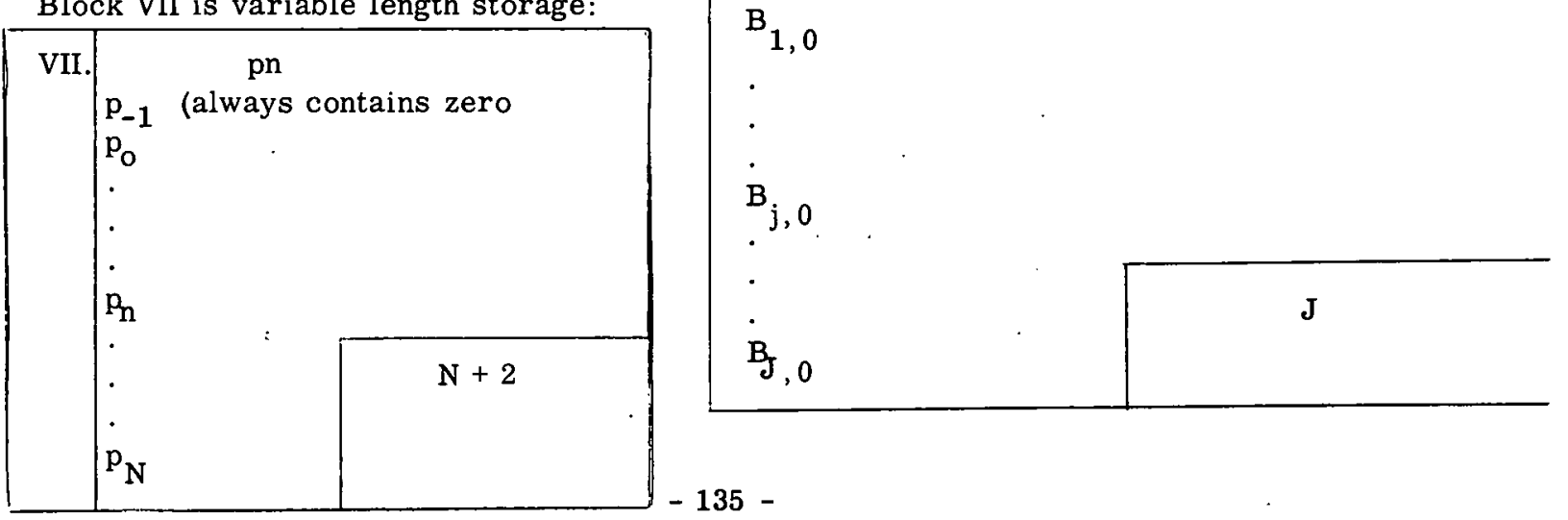




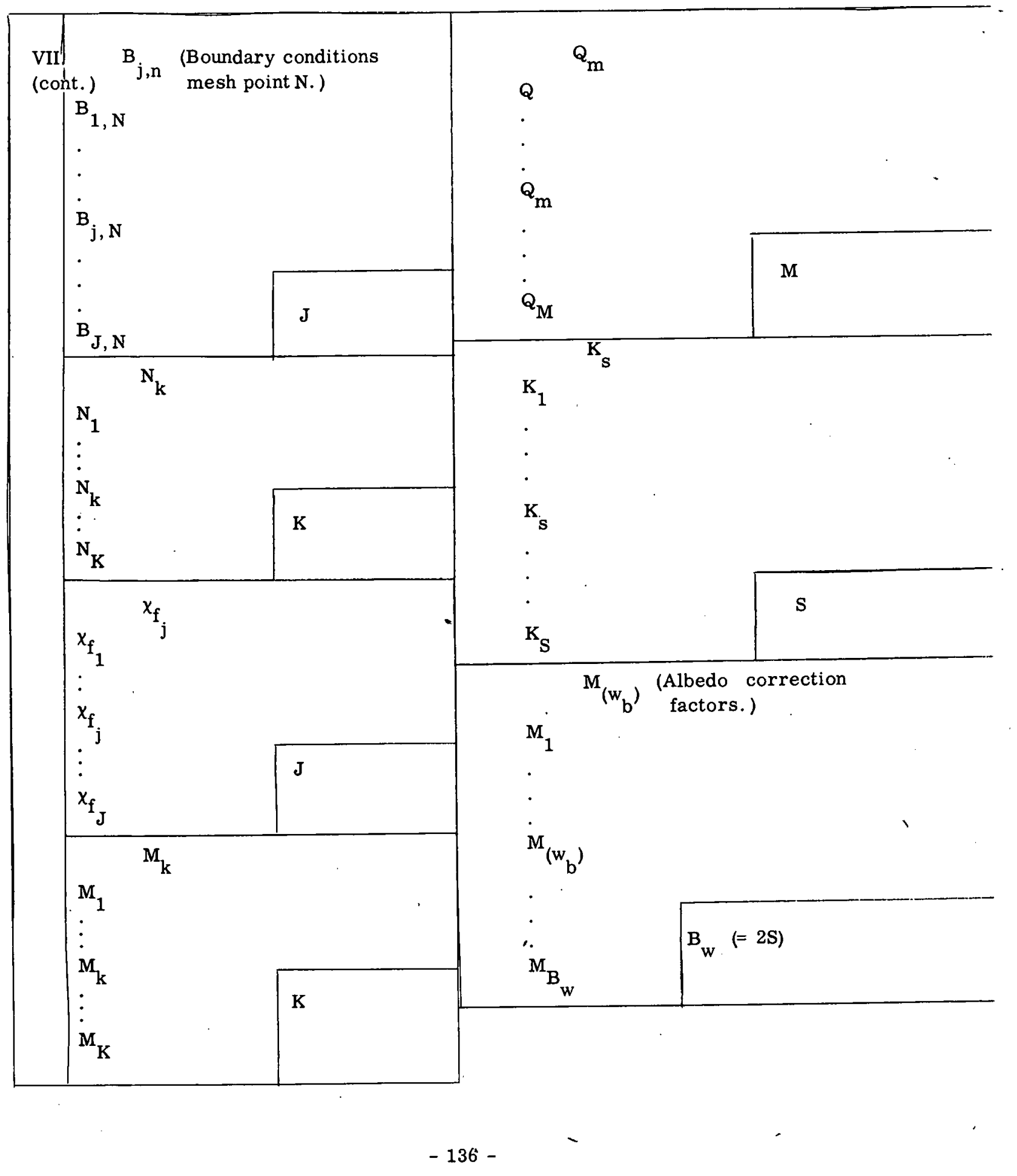




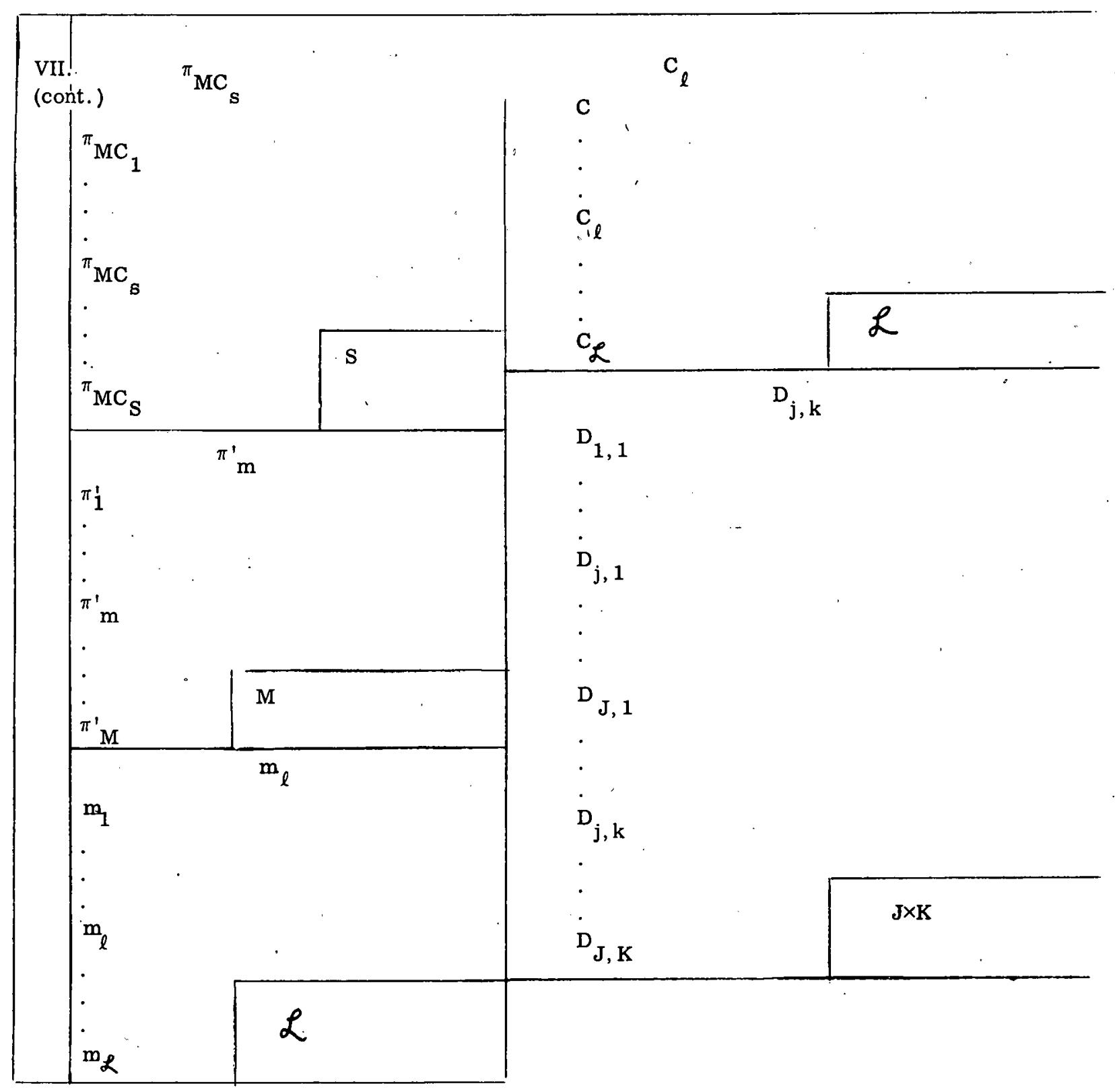




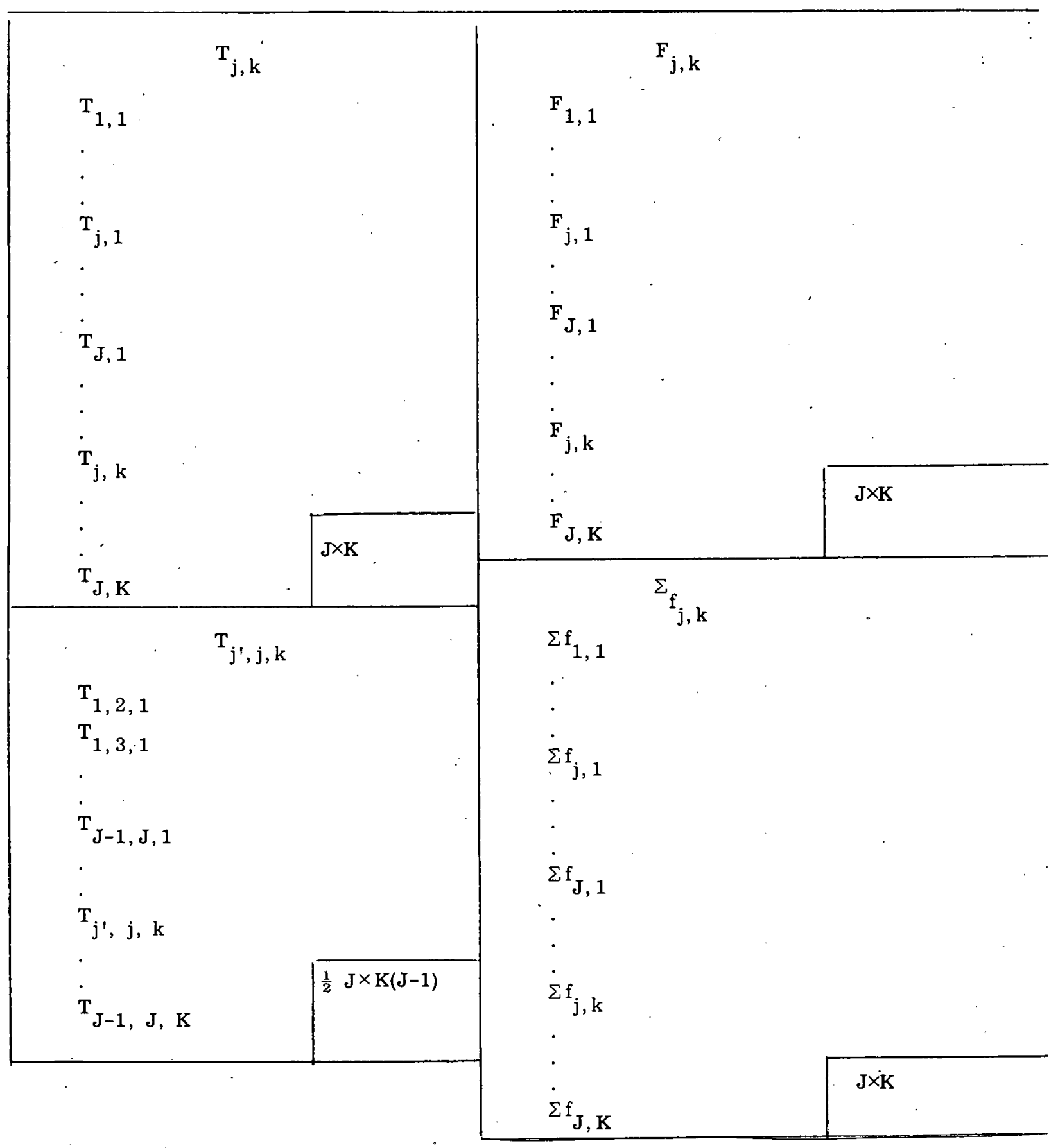




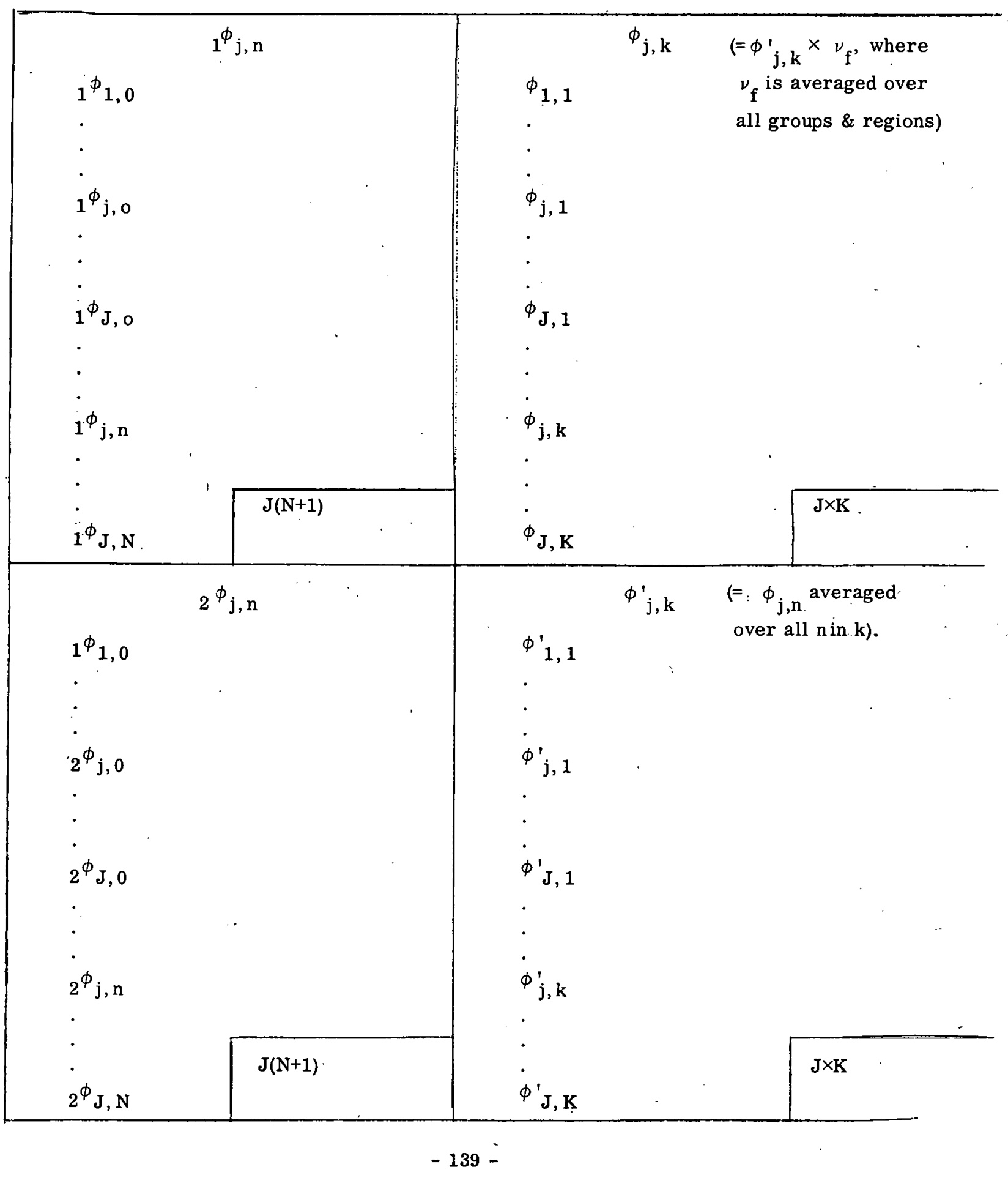




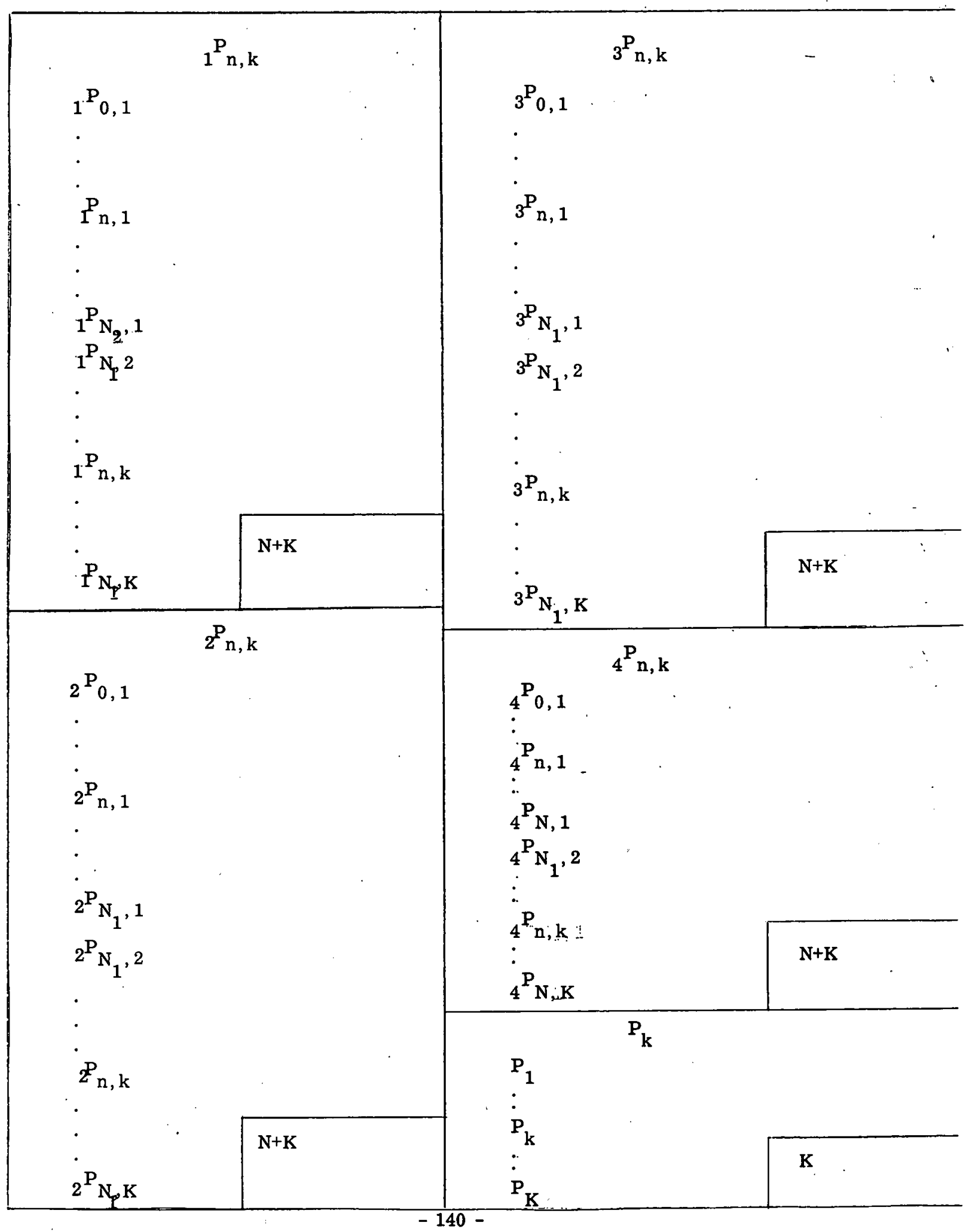




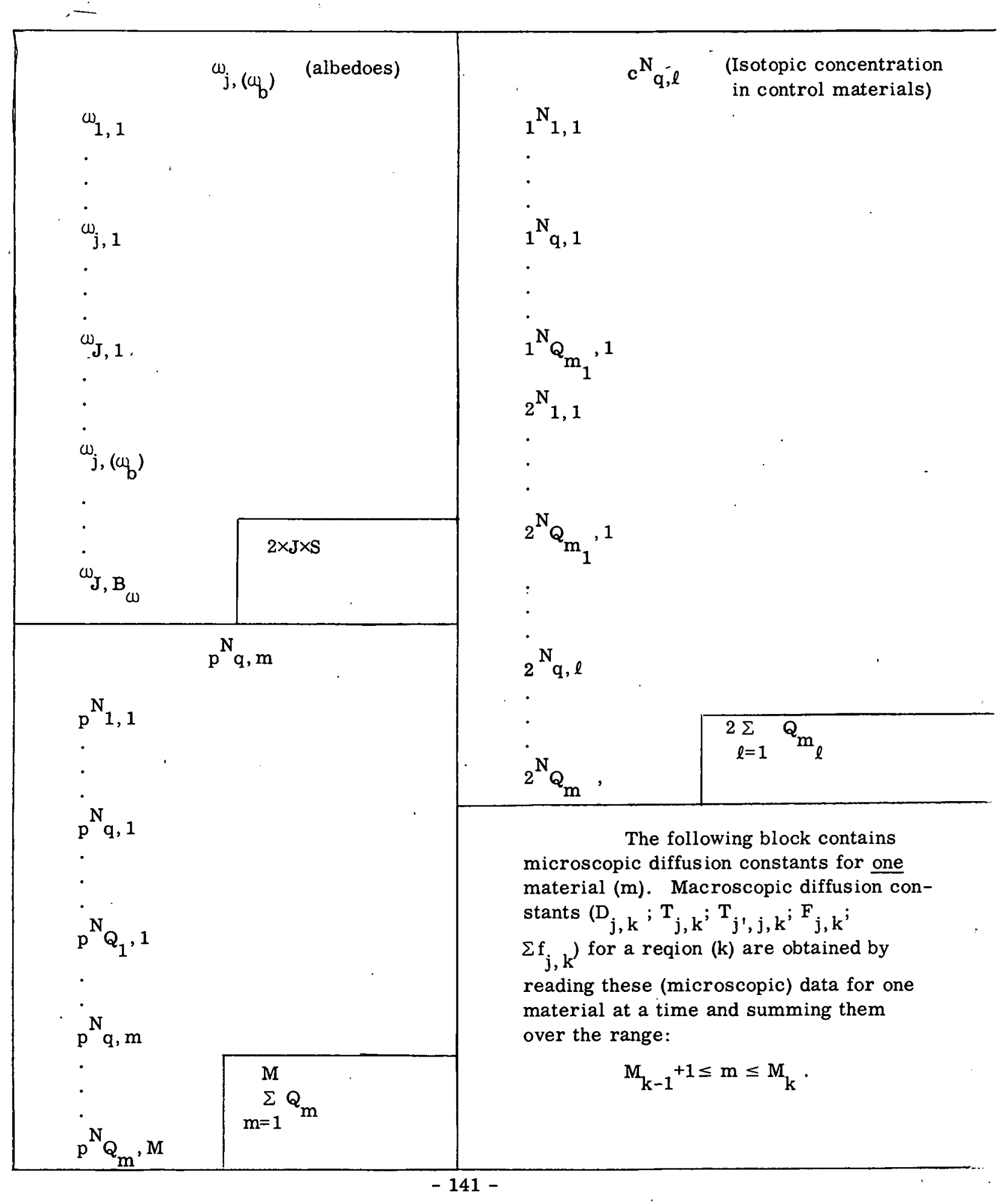




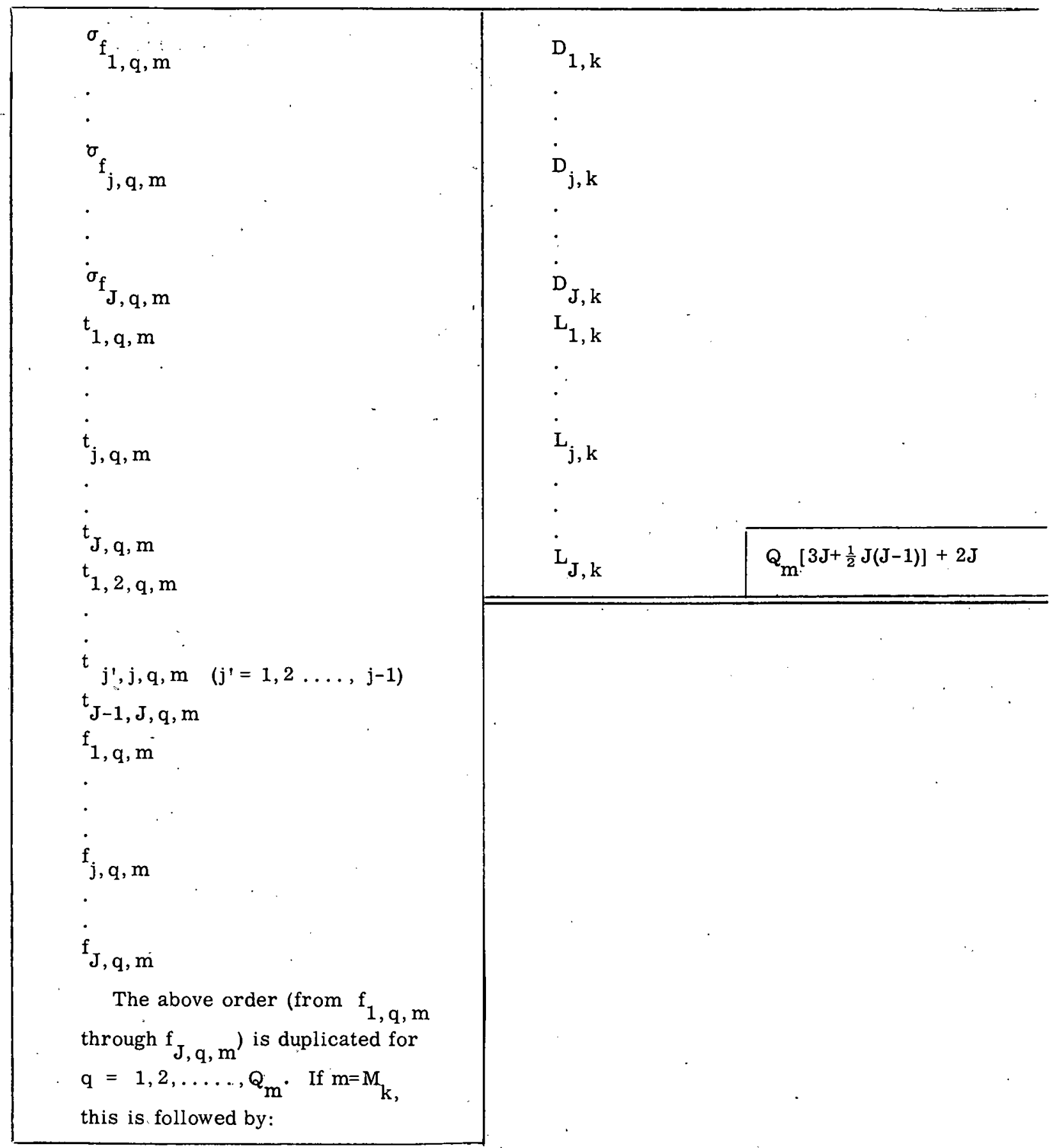


B. Burnup Code

I. RBU monitor

II. Coding - Burnup.

1. Pre-burnup

2. Burnup

3. Post-burning

III. Coding - Subroutines.

1. Tape dispatcher.

IV. Permanent burnup data.

1. Constants

2. $\mathrm{BCI}$

3. DSC commands

V. "Eraseable" storage.

11 words - intermediate results.

VI. "Common" storage Indirect address block and input data. 42 words. (LWA is "EPS2").

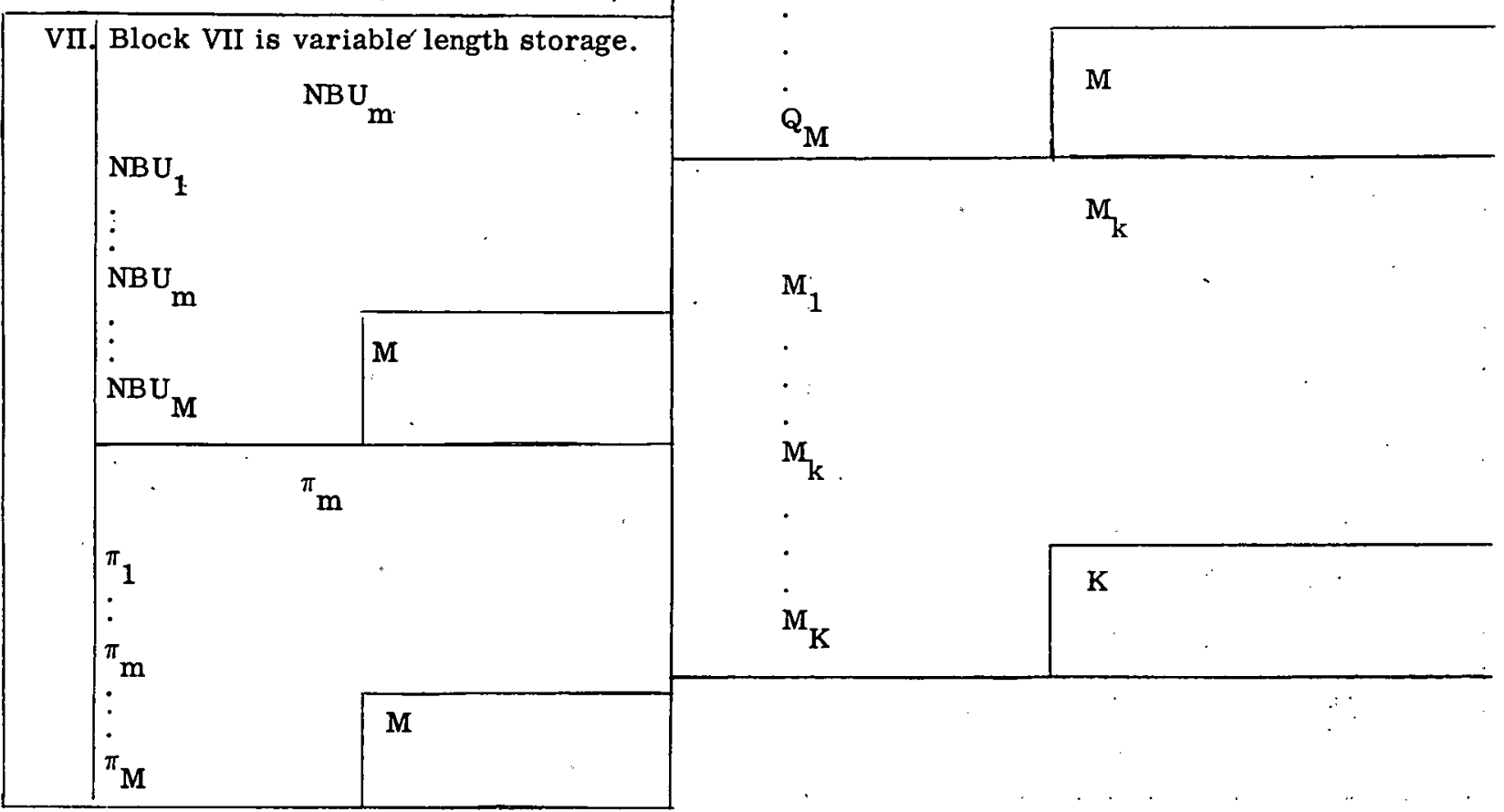




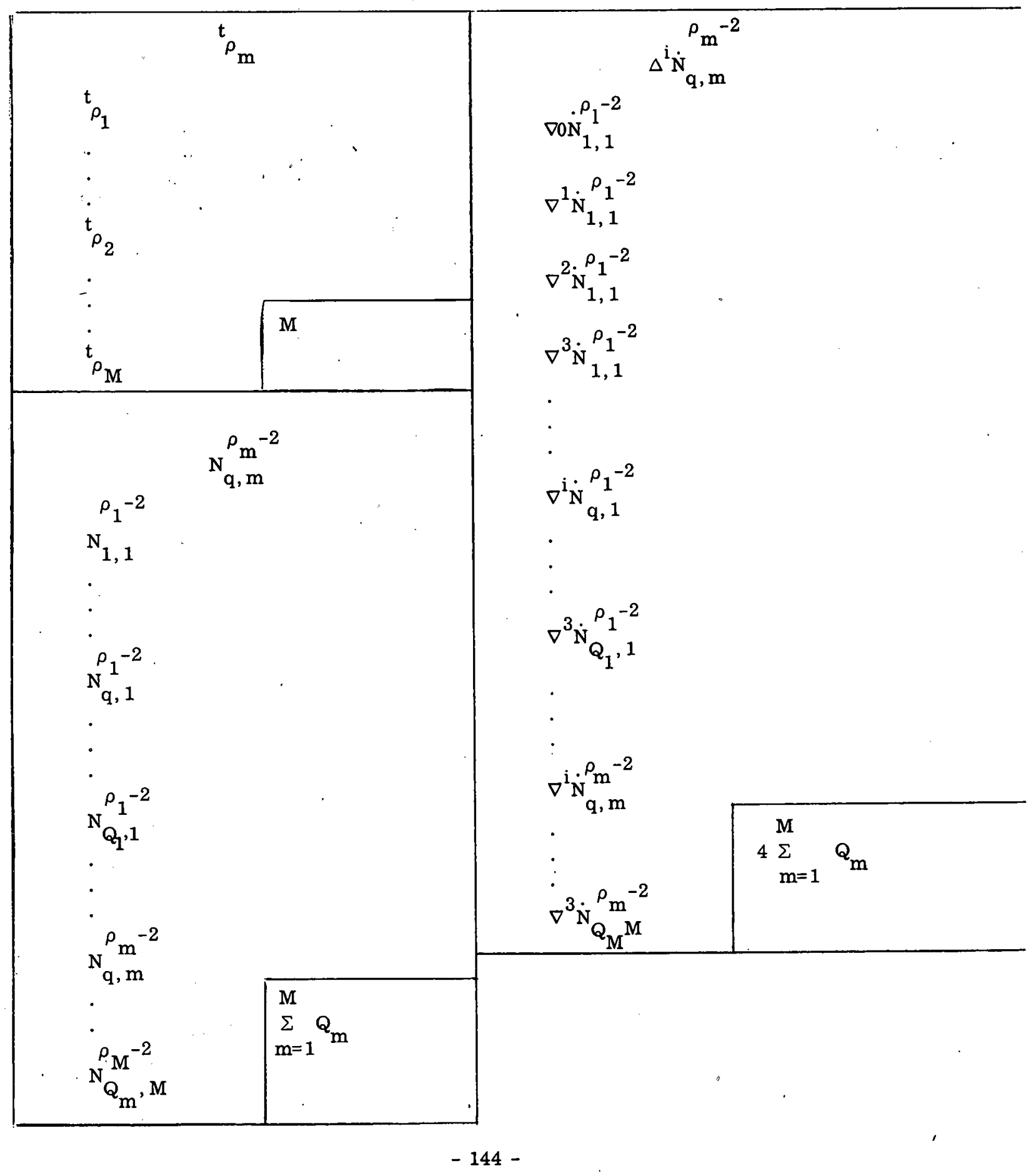




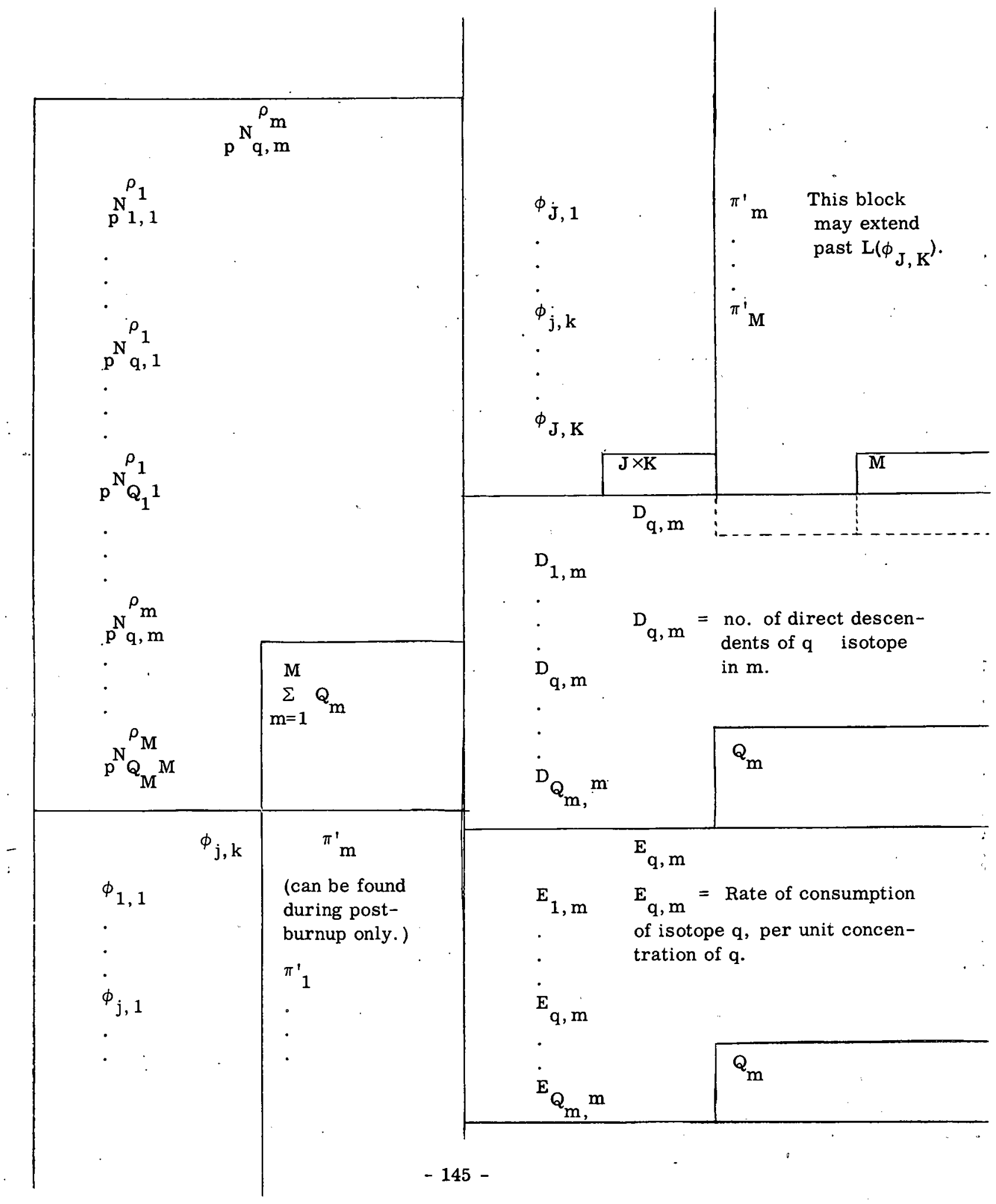




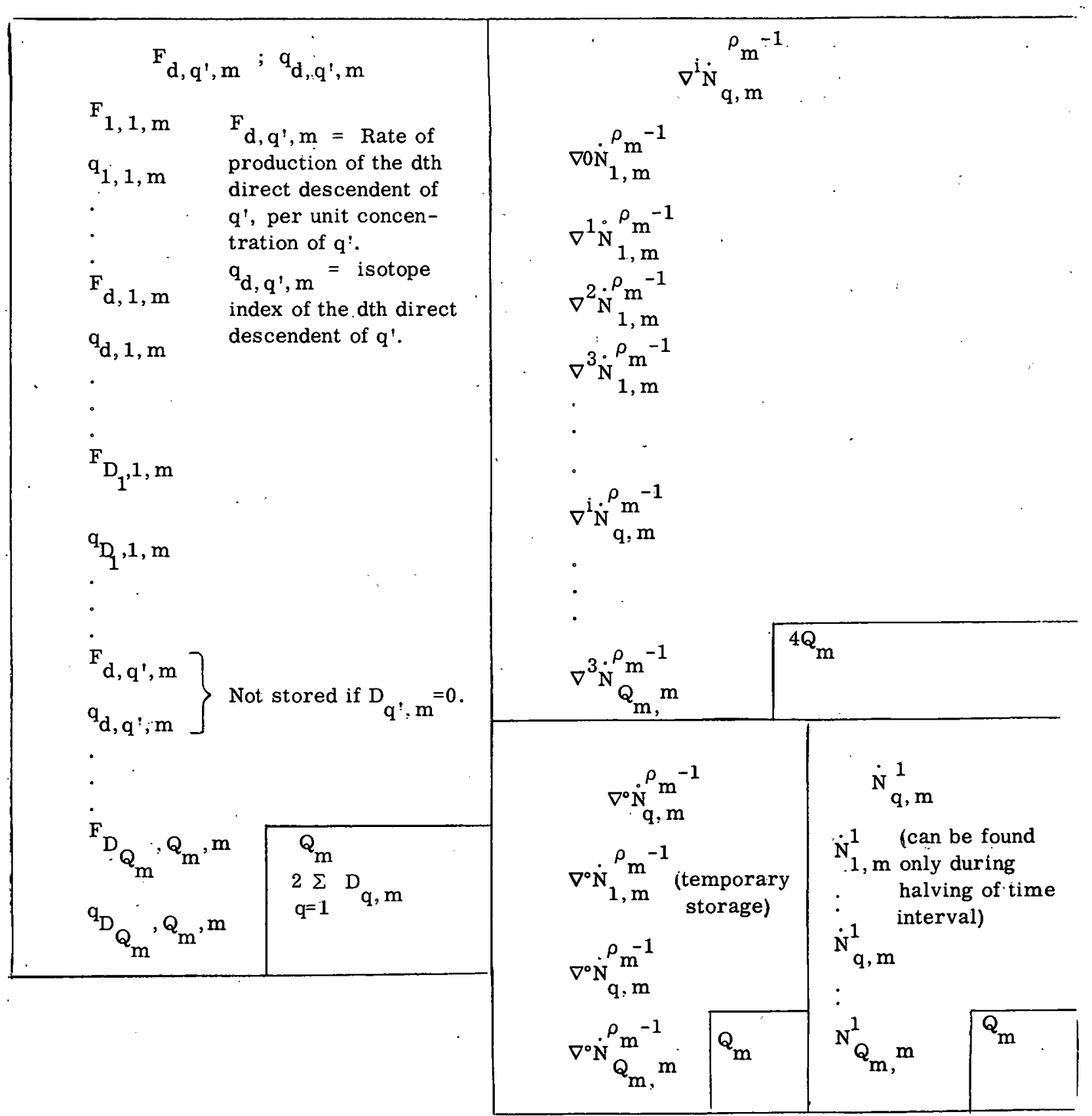




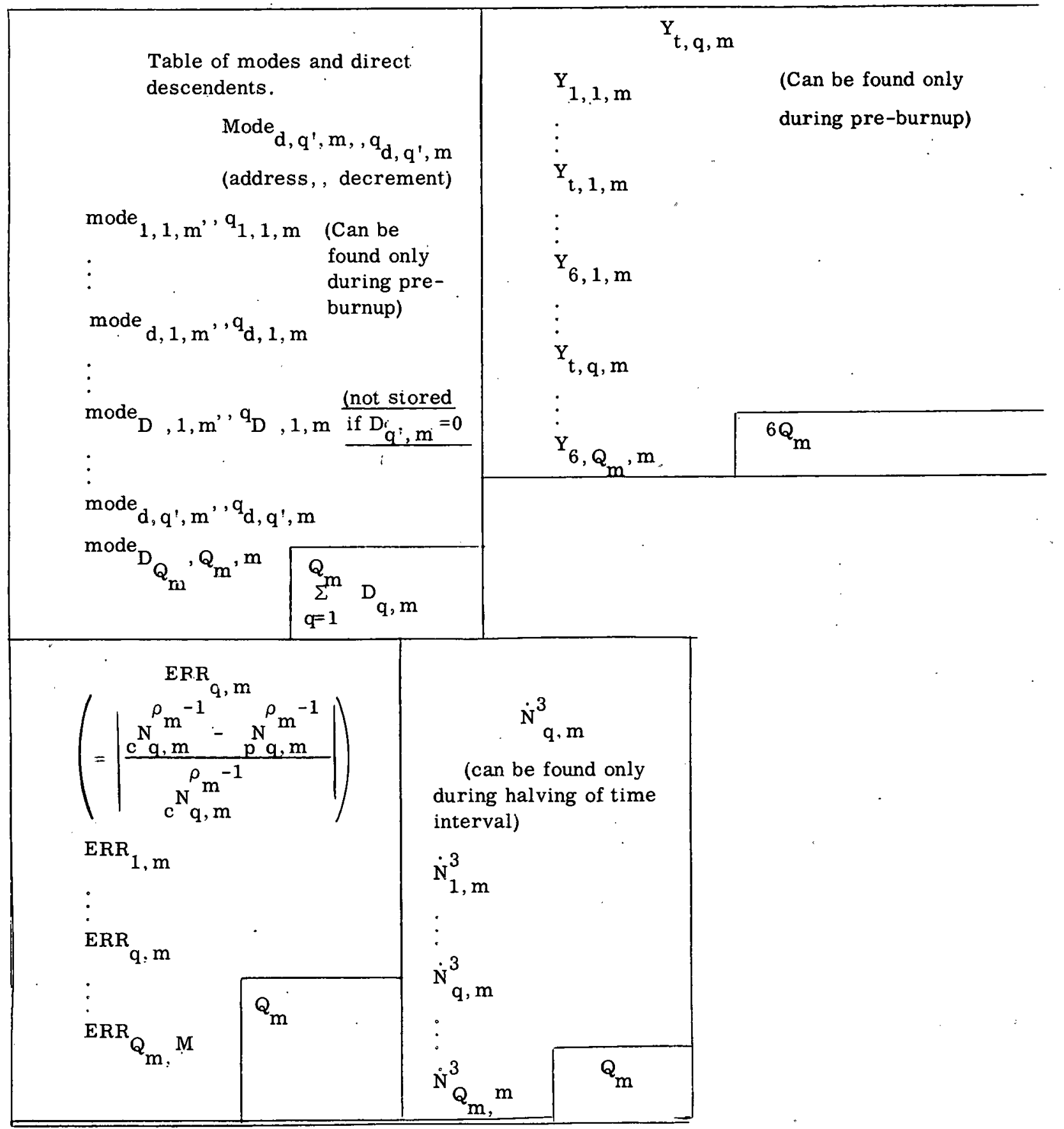




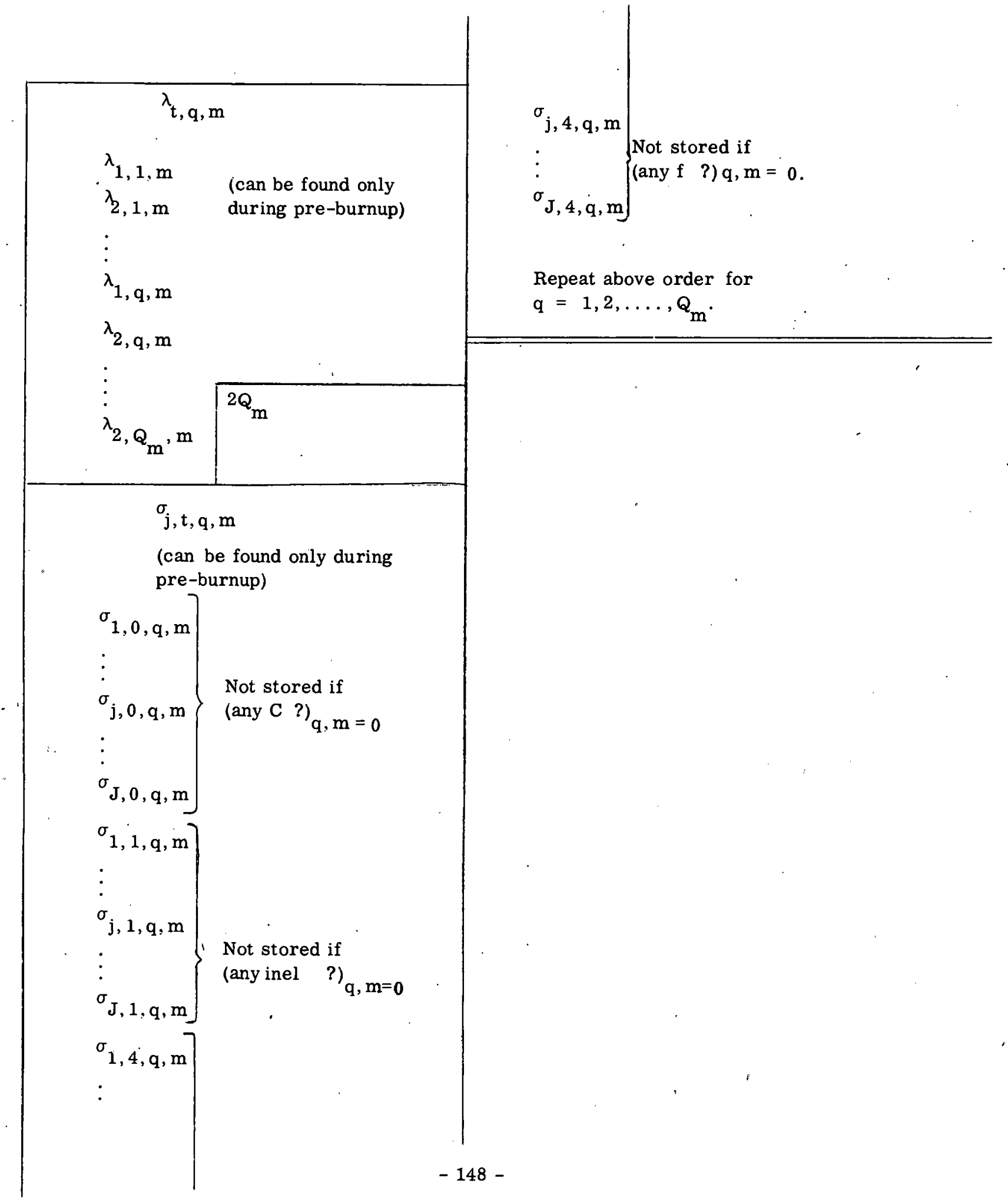


C. Monte Carlo

1. General for all Monte Carlo

RBU monitor

ORIGIN

Tape control and tally routines

Tape addresses and tape tally block addresses

Constants... (function of system and problem)

Addresses. . (function of system)

Fixed constants

Erasable block

STARI

DSC commands

Constants (variables)

Addresses

Fixed constants

Program

Sub routines

Working storage
This system is assembled and is contained in the pre-monte carlo record, and is read in to memory with PRE, to be used by the PRE, MC, and Post. The address block contains the addresses of all blocks from working stoppage to END.
The main part of each program begins here, i (stari) $=1$ for PRE-MC, I 2 for MC, = 3 for POST MC; STARI is a transfer to first instruction to be executed. The monte carlo and post MC read into memory starting at STARI. 
$\left.\begin{array}{l}\text { Input } \\ \text { Tally blocks } \\ \text { Temporary storage }\end{array}\right\}$

END

\section{Pre-Monte Carlo}

VIB

MSUBS

QSUBS

DNMC

PIMC

TRANB
See individual memory maps for this.

$\left(\mathrm{v}_{1}, \mathrm{v}_{2} ; \mathrm{v}_{\mathrm{I}}\right)$

$\left(M_{1}, M_{2}, \ldots M_{S}\right)$

$\left(Q_{1}, \dot{Q}_{2}, \cdots Q_{S}\right)$

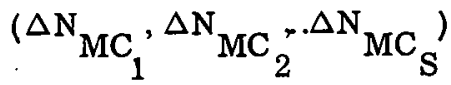

$\left(\pi{ }_{\mathrm{MC}_{1}}, \pi{ }_{\mathrm{MC}_{2}}, \ldots \pi \mathrm{MC}_{\mathrm{S}}\right)$

Used to transfer contents of MC1

to $\mathrm{MC} 2$ and $\mathrm{RB} 4$. 
3. Monte Carlo

$\mathrm{v}_{1}$
$\mathrm{v}_{2}$
$\cdot$
$\mathrm{v}_{\mathrm{I}}$

PFLAB

$$
\begin{array}{ll}
R_{1}^{\prime \prime} & \text { PFLB } \\
R_{2}^{\prime \prime} & \text { PFLB }+I \\
\vdots & \\
R_{M}^{\prime \prime} & \text { PFLB }+I(M-1) \\
& Q_{m} \\
\left(R_{m}^{\prime \prime} \equiv \sum_{1}(\text { any } r)_{q m}\right)
\end{array}
$$

PELAB $\quad R_{1} \quad$ PELB

$\mathrm{R}_{2} \quad \mathrm{PELB}+\mathrm{IR}_{\mathrm{o}_{1}}$

: $\quad \mathrm{M}-1$

$\mathrm{R}_{\mathrm{M}} \quad \mathrm{PELB}+\mathrm{I} \sum_{1} \mathrm{R}_{\mathrm{o}_{\mathrm{m}}}$

PINAB $R_{1}^{\prime} \quad$ PINB

$\begin{array}{lll}\mathrm{R}_{2}^{\prime} & \mathrm{PINB}+\mathrm{I} \mathrm{R}_{0}^{\prime} \\ & & \end{array}$

: $\quad$ :

$\mathrm{R}_{\mathrm{M}}^{\prime} \quad \mathrm{PINB}+\mathrm{I} \sum_{1}^{\mathrm{M}-1} \mathrm{R}_{0_{\mathrm{m}}^{\prime}}$

$$
\begin{aligned}
& A L A A B: \quad L C A B+R_{1} \quad A W A B+2 R_{0} \\
& \mathrm{LCAB}+\mathrm{R}_{1}+\mathrm{R}_{2} \quad \mathrm{AWAB}+2\left(\mathrm{R}_{1}+\mathrm{R}_{0}\right) \\
& \mathrm{LCAB}+\sum_{1}^{\mathrm{M}} \mathrm{R}_{\mathrm{m}} \mathrm{AWAB}+2 \sum_{1}^{\mathrm{M}} \mathrm{R}_{\mathrm{m}}
\end{aligned}
$$

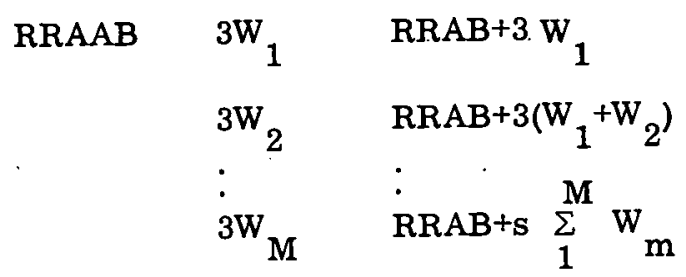

SDVAAB

SDVAB+R'

$S D V A B+R_{1}^{\prime}+R_{2}^{\prime}$

SDVAB $+\sum_{1}^{M} R_{m}^{\prime}$

LCAB
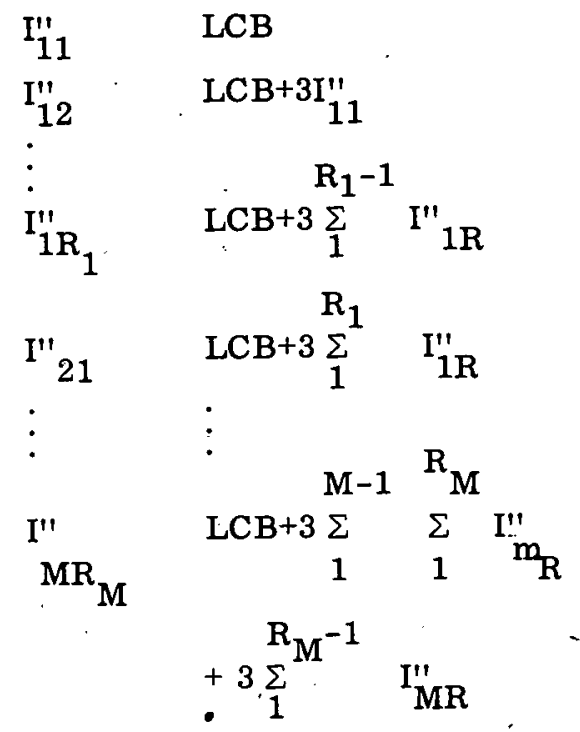


$$
\begin{aligned}
& \text { AWAB } \quad\left(I-R_{11}\right) \quad \text { AWB +\& } 11 \\
& \text { - PDVB+34( } \left.\varepsilon_{11}^{\prime}-1\right) \\
& \left(\mathrm{I}-\mathrm{R}_{12}\right) \quad \mathrm{AWB}+\mathcal{H}_{12} \\
& \text { - PDVB }-3\left(\varepsilon_{12}^{\prime}-1\right) \\
& \left(\mathrm{I}-\mathrm{R}_{\mathrm{m}_{\mathrm{R}}}\right) \mathrm{AWB}+\boldsymbol{\mu}_{\mathrm{mR}} \\
& -\operatorname{PDVB}-34\left(\varepsilon_{m R}^{\prime}-1\right) \\
& \begin{array}{ll}
\vdots & \\
{ }_{M}^{I-R_{M}} & A W B+\not{ }_{M R} \\
& -P D V B-34\left(\xi_{M R_{M}}^{\prime}-U\right)
\end{array} \\
& \text { SDVAB } \left.\left.\quad \text { - [ - PVINB-34(s }{ }_{11}^{\prime}-1\right)\right] A W B+\mathfrak{S}_{11} \\
& \text { or } s_{11}^{\prime} \\
& \text { - [ - PVINB-34(s's } \left.\left.{ }_{12}^{\prime}-1\right)\right] A W B+\mathscr{S}_{12} \\
& \text { or } \quad s_{12}^{\prime} \text { : } \\
& -\left[-\operatorname{PVINB}-34\left(\mathrm{~s}_{\mathrm{MR}{ }_{\mathrm{M}}^{\prime}}^{\prime}-1\right)\right] \mathrm{AWB}+{ }_{\mathrm{MR}}{ }_{\mathrm{M}} \\
& \text { RRAB } \\
& \begin{aligned}
-\left(\operatorname{RRB}+\rho_{11}\right) & -\mathrm{RRTB} \\
\mathrm{N}_{11} & \\
-\left(\mathrm{RRB}+\rho_{12}\right) & - \text { RRTB }-3 \mathrm{~J} \\
\mathrm{~N}_{12} &
\end{aligned}
\end{aligned}
$$

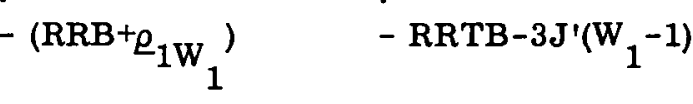

$$
\begin{aligned}
& \mathrm{N}_{1 \mathrm{~W}_{1}}
\end{aligned}
$$

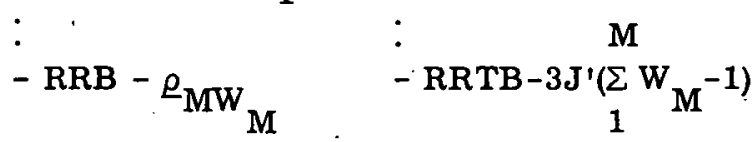

$$
\begin{aligned}
& -152- \\
& \text { : } \quad \text { : } \\
& \left(\mathrm{R}_{M}^{\prime}-1\right)^{\mathrm{P}_{\text {IN }}}{ }_{\mathrm{MI}}^{\mathrm{R}_{M}^{\prime}} \mathrm{P}_{\mathrm{MI}}
\end{aligned}
$$




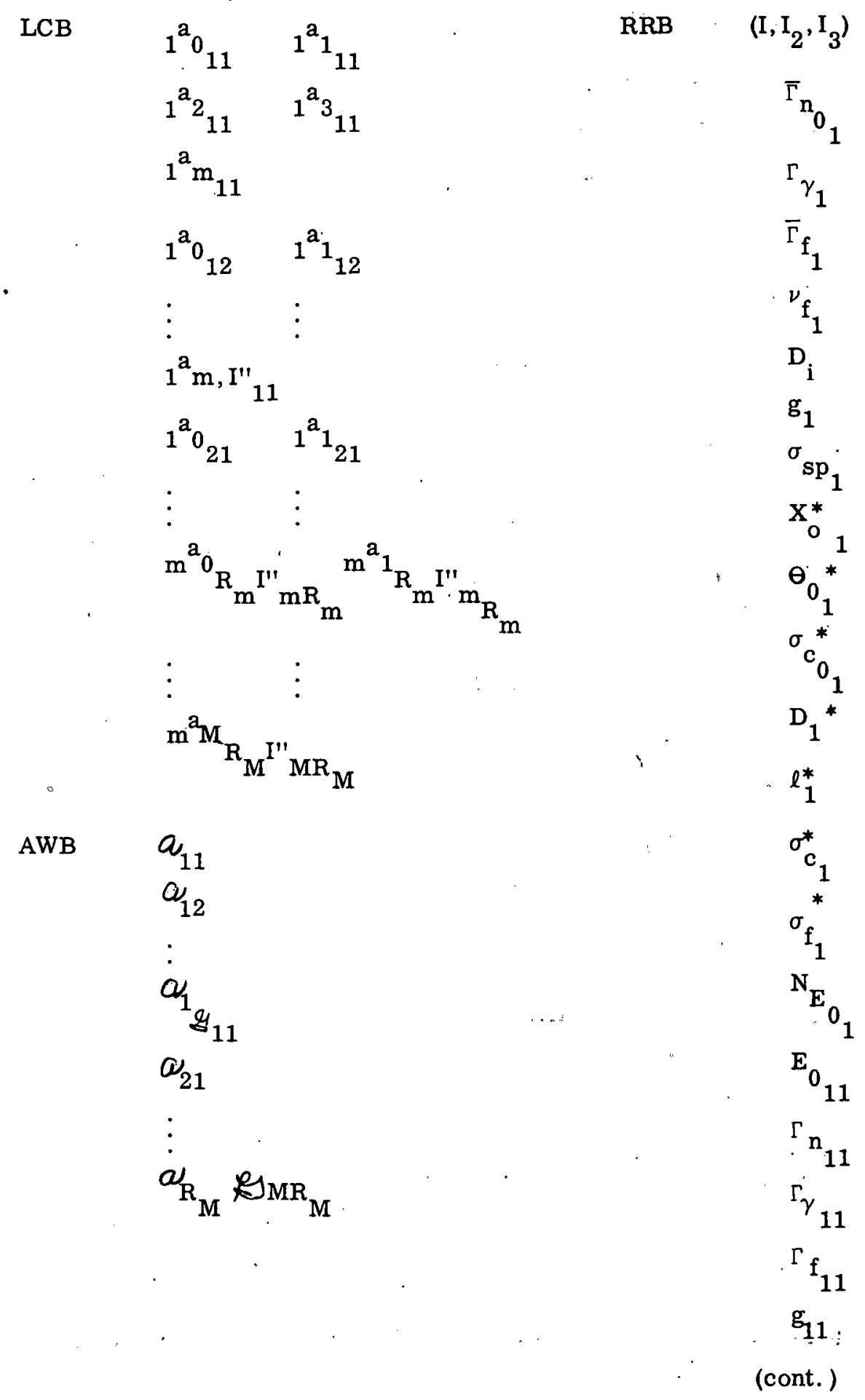



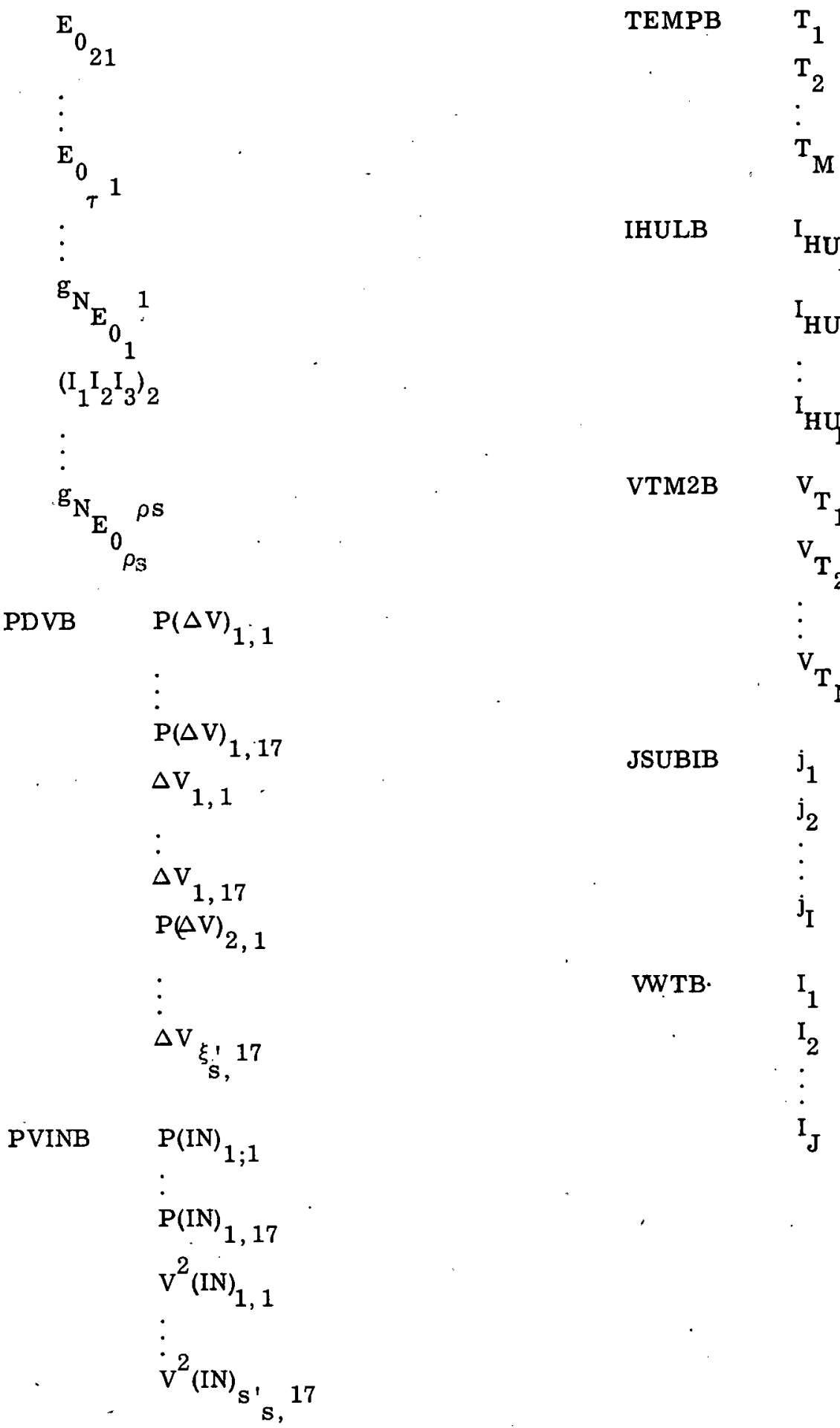

IHULB
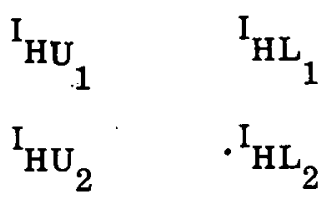

:
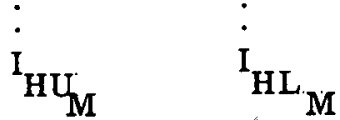

VTM2B

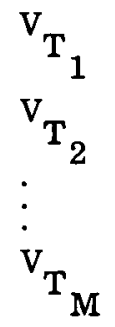

JSUBIB

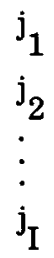

VWTB

$$
\begin{aligned}
& \mathrm{I}_{1} \\
& \mathrm{I}_{2} \\
& \vdots \\
& \mathrm{I}_{\mathrm{J}}
\end{aligned}
$$



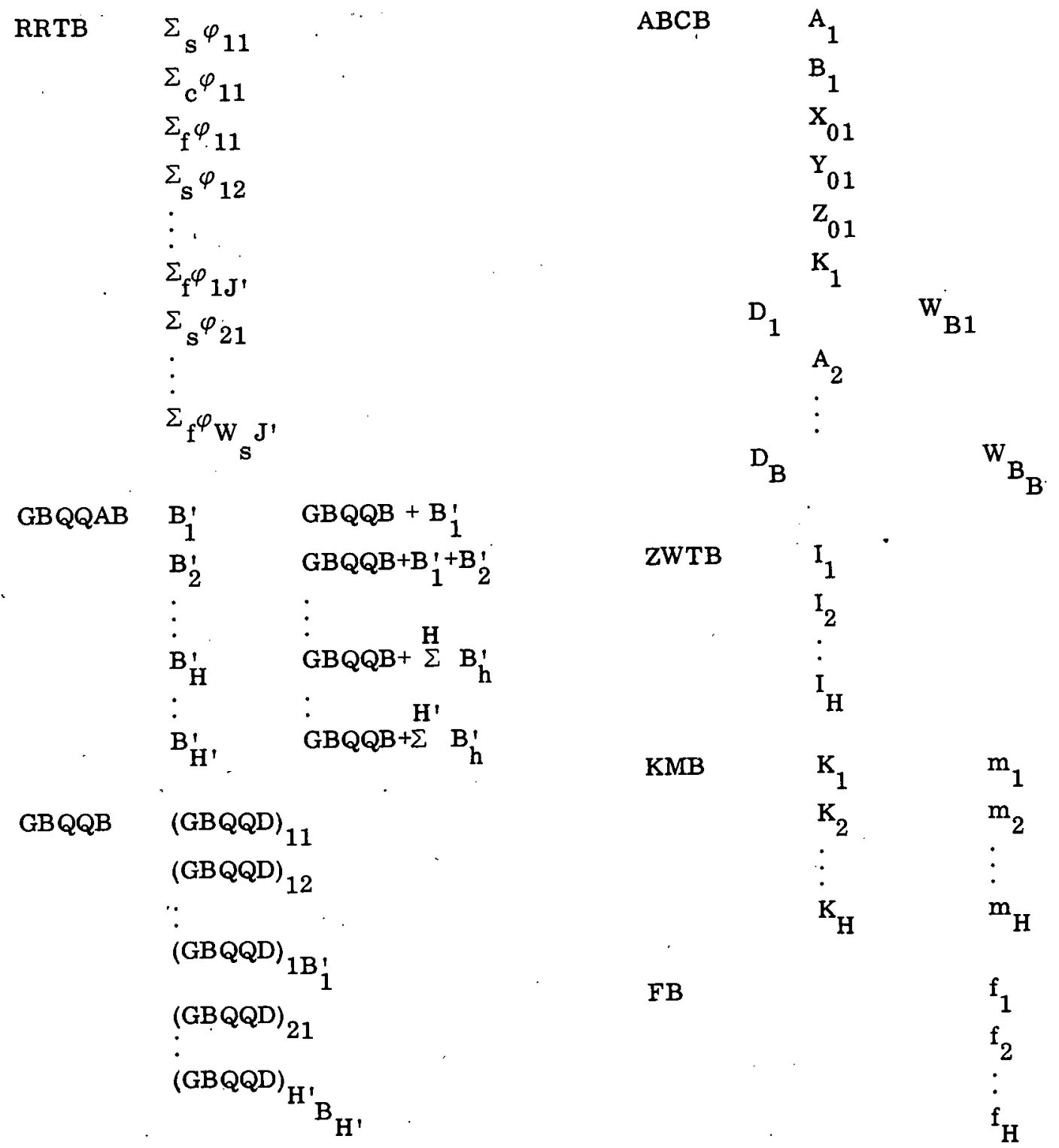


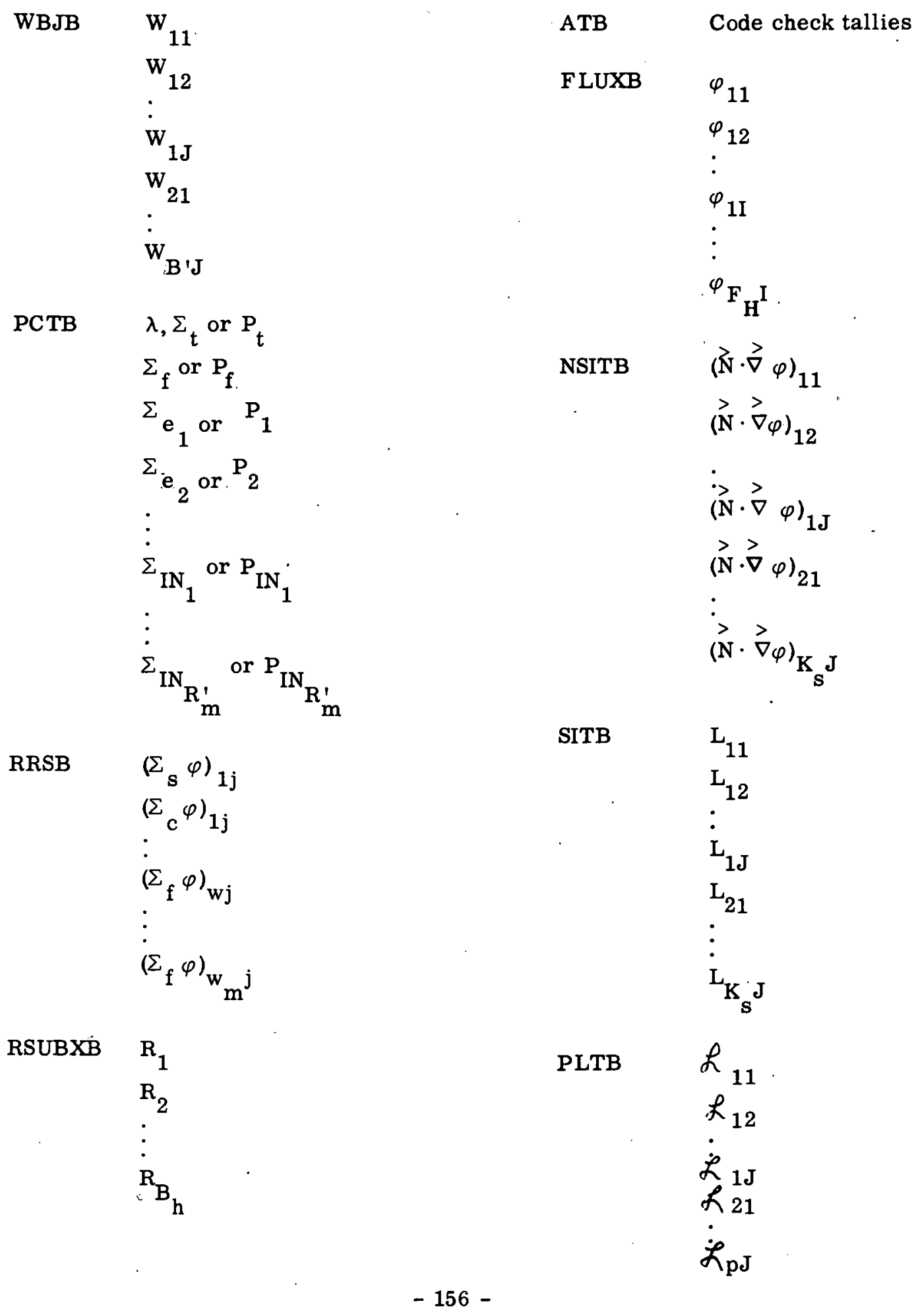



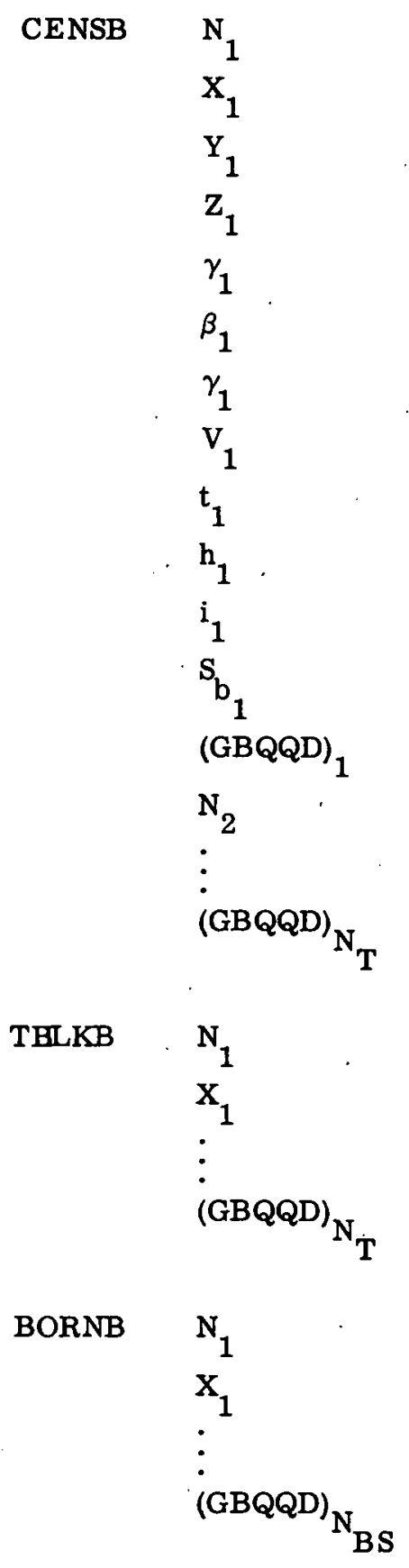


\section{Post Monte-Carlo}

\begin{tabular}{|c|c|c|c|}
\hline VIB & $\begin{array}{l}\mathrm{V}_{\mathrm{i}} \\
\underline{\mathrm{I} \text { Wds. }}\end{array}$ & PLTB & $\begin{array}{l}\text { Perpendicular leakage } \\
\text { tallies. } \\
\mathrm{J} \times \mathrm{K} \text { wds. }\end{array}$ \\
\hline RRCB & $\begin{array}{l}\text { Random resonance } \\
\text { Isotopic concentra- } \\
\text { tions. }{ }^{w_{S} \text { wds. }}\end{array}$ & EIB & $\begin{array}{l}E_{i} \\
\text { I wds. }\end{array}$ \\
\hline JSUBIB & $\begin{array}{l}\mathrm{j}_{\mathrm{i}} \\
\underline{\mathrm{I} \text { wds: }}\end{array}$ & EJB. & $\begin{array}{l}\mathbf{E}_{\mathbf{j}} \\
\text { J wds. }\end{array}$ \\
\hline RRTB & $\begin{array}{l}\text { Random resonance } \\
\text { tallies. } \\
3 \times \mathrm{J}^{\prime} \times \mathrm{w}_{\mathrm{s}} \text { wds. }\end{array}$ & ${ }^{*} \mathrm{ACFB}$ & $\begin{array}{l}\text { Acceleration factors } \\
I \times F_{S} \text { wds. }\end{array}$ \\
\hline KMB & $\begin{array}{l}\mathrm{K}_{\mathrm{h}} \text { in decrement. } \\
\mathrm{m}_{\mathrm{h}} \text { in address. } \\
\mathrm{H} \text { wds. }\end{array}$ & * TBLKB & $\begin{array}{l}\text { Tape block } \\
13 \times \mathrm{N}_{\mathrm{T}} \text { wds. }\end{array}$ \\
\hline סחא & $\mathrm{f}_{\mathrm{h}}$ & ${ }^{*}$ CENSB & $\begin{array}{l}\text { Census block } \\
13 \times \mathrm{N}_{\mathrm{T}} \text { wds. }\end{array}$ \\
\hline FB & $\mathrm{H}_{\mathrm{S}}$ wds. & ISO1 B & Isotope input block \\
\hline FLUXB & $\begin{array}{l}\text { New M.C. fluxes } \\
\mathrm{F}_{\mathrm{S}} \times \mathrm{I} \text { wds. }\end{array}$ & $\begin{array}{l}(\text { ISO1A }= \\
A C F A)\end{array}$ & 1. \\
\hline NSITB & $\begin{array}{l}\text { Normal surface } \\
\text { integral tallies: } \\
\mathrm{J} \times \mathrm{K} \text { wds. }\end{array}$ & ISO2B & $\begin{array}{l}\text { Isotope input block } \\
2 .\end{array}$ \\
\hline SITB & $\begin{array}{l}\text { Surface intégral } \\
\text { tallies. }\end{array}$ & FLIMB & $\begin{array}{l}\varphi_{\text {im }} \\
\text { I words }\end{array}$ \\
\hline & $\mathrm{J} \times \mathrm{K}$ wds. & - EAJB & $f\left(\alpha_{A_{1}} j\right)$ \\
\hline & & & J wds. \\
\hline
\end{tabular}

* Present until end of acceleration. 
$E_{j-1}-E_{j}$
J wds.

$\left(E_{j-1}-E_{j)} \sum_{f \text { in } k} \sum_{i \text { in } j} \ddot{\varphi}_{i f}\right.$

Jwds.

Random resonance

RRNB normalizers.

J words

IAJB i $(\alpha j)$

J wds.

Microscopic

diffusion constants.

DIFB

$Q_{m}\left[3 J+\frac{1}{2} J(J-1)\right]$ wds.

\section{Microscopic burnup}

constants.

Q $\mathbf{m}$

$\mathrm{J} \sum_{\mathrm{q}=1}\left[\left(\begin{array}{ll}\text { any } & \mathrm{C}\end{array}\right)_{\mathrm{q}}+\right.$

BURPB

$\left(\text { any }{ }^{\mathrm{F}}\right)_{\mathrm{q}}+$

(any INE L) ${ }_{q}$ ] 
XVI. LIST OF INPUT DATA (TENTATIVE)

A. Material and Energy Group Set Data

$\mathrm{M}_{\mathrm{O}} \quad$ Total number of basic materials

$i \quad$ Microscopic energy group set number

$I_{i} \quad$ Number of microscopic groups ( 0 if set $i$ used before)

M Total number of actual materials

$\left.\begin{array}{l}\mathrm{m}_{01} \\ \cdot \\ \mathrm{m}_{\mathrm{O}_{\mathrm{O}}}\end{array}\right\}$ Basic material names

$\left.\begin{array}{l}\underline{l}_{1} \\ \cdot \\ \underline{\ell}_{\mathrm{M}_{0}}\end{array}\right\}$ Unity if control materials are associated with basic material $\mathrm{m}_{\mathrm{o}}$, zero if not

$\left.\begin{array}{l}\mathbf{Q}_{01} \\ \cdot \dot{Q}_{\mathrm{OM}}\end{array}\right\}$ Number of input isotopes in each basic material

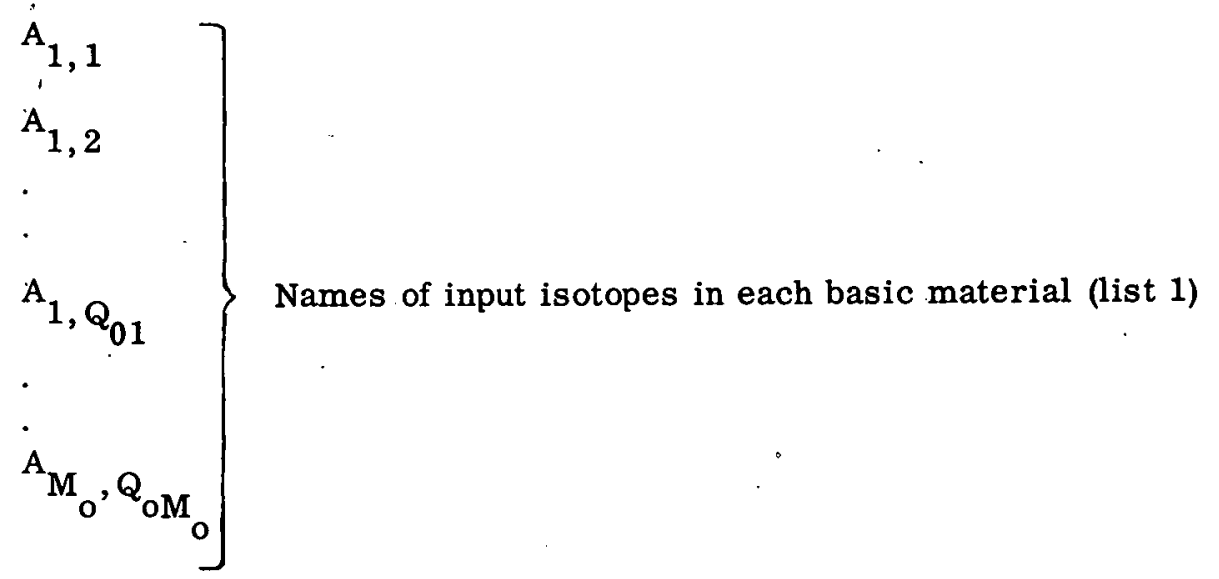




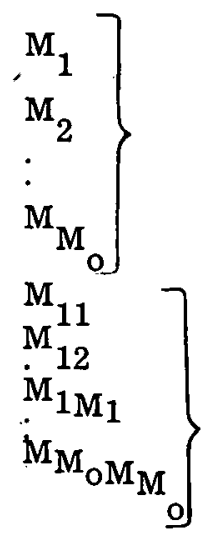

Number of actual materials associated with each basic material

Names of actual materials (grouped by basic materials in order)

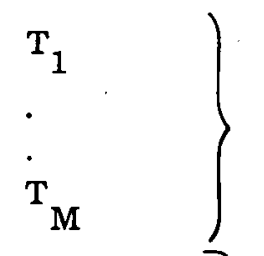

Temperature of each actual material, ${ }^{\circ} \mathrm{C} / 10$ (rounded to nearest multiple of $10^{\circ}$ and divided by 10 )

$\mathrm{g}_{\mathrm{A}_{1}, \mathrm{~m}_{11}}$

$\mathrm{g}_{\mathrm{A}_{1}, \mathrm{~m}_{12}}$

Special Atomic Weight Table Names

List order: for each isotope entry in List 1 , list the table names corresponding to all actual materials in the basic material involved $\cdot$ in that isotope entry in List 1.

-

$\mathrm{g}_{\mathrm{A}_{1}, \mathrm{~m}_{1 \mathrm{~m}_{1}}}$ $\left.\mathrm{g}_{\mathrm{A}_{2}} ; \mathrm{m}_{21}\right]$

-

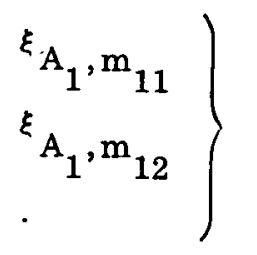

Velocity Increment Probability Table Names (list by materials for each isotope entry in list 1 , as for $g_{A, m}$ )

$\left.\begin{array}{l}\mathbf{K}_{1} \\ \cdot \\ \mathbf{K}_{\mathbf{m}} \\ \cdot \\ \dot{K}_{\mathbf{M}}\end{array}\right\}$

Macroscopic region name for each actual material 


$$
\left.\begin{array}{l}
\mathrm{E}_{\mathrm{th} 1} \\
\mathrm{E}_{\mathrm{thm}} \\
\cdot \\
\mathrm{E}_{\mathrm{thM}} \\
\mathrm{E}_{1_{i}} \\
\dot{\mathrm{E}}_{\mathrm{I}_{i}}
\end{array}\right\}
$$

$\mathrm{G}_{\mathrm{o}}$

$\mathrm{g}_{\mathrm{o}} \quad$ Name of a special atomic weight table

$b_{\text {go }}$

$a_{1, \text { go }}$

$$
\dot{a}_{g_{\text {go }}, \text { go }}
$$

Maximum neutron energy to which thermal velocity increments will be added, for each actual material

Upper limits of microscopic groups (missing if groûp set $i$ was used before

Number of special atomic weight tables

Number of atomic weights in $\mathrm{g}_{\mathrm{o}}$

Atomic weight for group $\dot{I}_{i}-g_{\text {go }}+1$

Repeat for each special atomic weight table from go to $a_{\&_{\text {go, }}}$ go

三 Number of velocity increment tables

$\xi \quad$ Name of a velocity increment table

$\left.\mathrm{P}_{1}\right] \quad$ Probabilities for table $\xi$.

. $3 \quad P_{1}=1.0, P_{17}=0$

$\mathrm{P}_{17}$

$\Delta \mathrm{V}_{1}$

- $\left\{\right.$ Velocity increments for table $\xi . \Delta v_{n} \geq \Delta v_{n+1}$

17]

Repeat for each velocity increment table 
$\mathbf{S}$

$\mathrm{M}_{1}$

$\mathrm{M}_{2}$

-

$\mathrm{M}_{\mathrm{S}}$

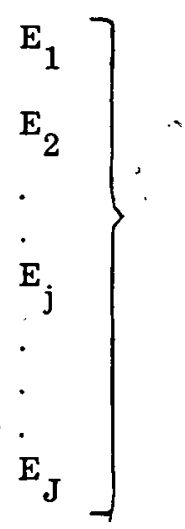

$\mathrm{N}_{11}$

$\mathrm{N}_{21}$

.

$\cdot$

$\cdot$

$\cdot$

$\mathrm{N}_{\mathrm{Q}_{01} 1}$

${ }_{1} \mathrm{~N}_{11}$

${ }_{1} \mathrm{~N}_{21}$

-

.

${ }_{1}{ }^{N} Q_{01} 1$

$2^{\mathrm{N}_{11}}$

$2^{\mathrm{N}} \mathrm{Q}_{01} 1$
Number of monte carlo systems

Highest material number in system 1

Upper energy limits of macroscopic energy groups

Concentration of isotopes in first basic material (if $\ell \neq 0$ this is for current mixture)

Concentration for $\mathrm{P}_{\ell}=0$ and $\mathrm{P}_{\ell}=1$ if $\underline{\ell} \neq 0$ for this material 
Repeat for each basic material

$\dot{\mathrm{N}}_{\mathrm{QM}^{\mathrm{M}}}$

B. Geometric Information

$\mathrm{H}_{\mathrm{S}}$

$\mathrm{K}_{\mathrm{S}}$

$T_{\text {OO }}$

$\mathbf{T}_{\mathrm{o}}$

$\mathbf{T}$

$\mathrm{H}^{\prime}$

$\mathbf{F}$

B

h

$\mathrm{B}_{\mathrm{h}}$

$\mathrm{b}_{1 \mathrm{~h}}$

g

q

$\mathbf{q}^{\prime}$

$\perp$

$\mathrm{L}$

$\mathrm{m}_{\mathrm{h}}$

$\mathrm{k}_{\mathrm{h}}$
Number of microscopic regions inside system $\mathbf{s}$ Highest macroscopic region number in system $s$

Time used in monte carlo an system $s$ to es tablish equilibrium in first time step

Time used in monte carlo in system $s$ to establish equilibrium in all time steps other than the first

Census time for system $s$ (time during which tallying takes place)

Number of microscopic regions for system $s$ including those used to define the outside of $s$

Number of region sets in system $\mathbf{s}$

Number of boundaries used in defining the microscopic regions of system $\mathbf{s}$

Microscopic region number

Number of boundaries used to define region $h$

Index of first boundary used to define region $h$

Sense of that boundary with respect to region $h$

Region into which particle leaving region $h$ through boundary $b_{1 h}$ is most likely to go

Next most likely region into which it might go; zero if only one possibility

Zero if particles leaving $h$ through $b_{1 h}$ do not contribute to perpendicular buckling; non zero if they do

Importance of region $\mathrm{h}$

Material used in region $h$

Macroscopic region containing region $h$ 
$\mathrm{f}_{\mathrm{h}}$

$\left.\begin{array}{c}h \\ B_{h} \\ \cdot \\ \cdot\end{array}\right\}$

$-\mathrm{b}$

A

B

C

$\mathrm{x}_{\mathrm{o}}$

Y

Z

K

d

$\mathrm{w}_{\mathrm{b}}$

$\left.\begin{array}{l}b \\ A \\ B \\ \cdot \\ \cdot \\ \cdot \\ \cdot \\ I_{1} \\ I_{2} \\ \cdot \\ \cdot \\ I_{J}\end{array}\right\}$

Region set containing region $h$

Repeat for each region of system $s$

For outer regions $q, q^{\prime}$ and $\perp$ are zero and $I_{h} m_{h} k_{h}$ and $f_{h}$ are not listed

Boundary index

Parameters in boundary equation

$\mathrm{A}\left(\mathrm{X}-\mathrm{X}_{\mathrm{O}}\right)^{2}+\mathrm{B}\left(\mathrm{Y}-\mathrm{Y}_{\mathrm{o}}\right)^{2}+\mathrm{C}\left(\mathrm{Z}-\mathrm{Z}_{\mathrm{o}}\right)^{2}-\mathrm{K}=0$

Boundary type. If this is such that some of the above are not required, they are ignored.

0 if this boundary does not reflect neutrons; if it does $w_{b}$ is the index of the set of albedoes to use. There are $2 \mathrm{~S}$ sets of albedoes computed by the post diffusion, one for each side of each system. The monte carlo need not use all of these.

Repeat for each boundary

Importances of macroscopic energy groups in system $\mathbf{s}$ 


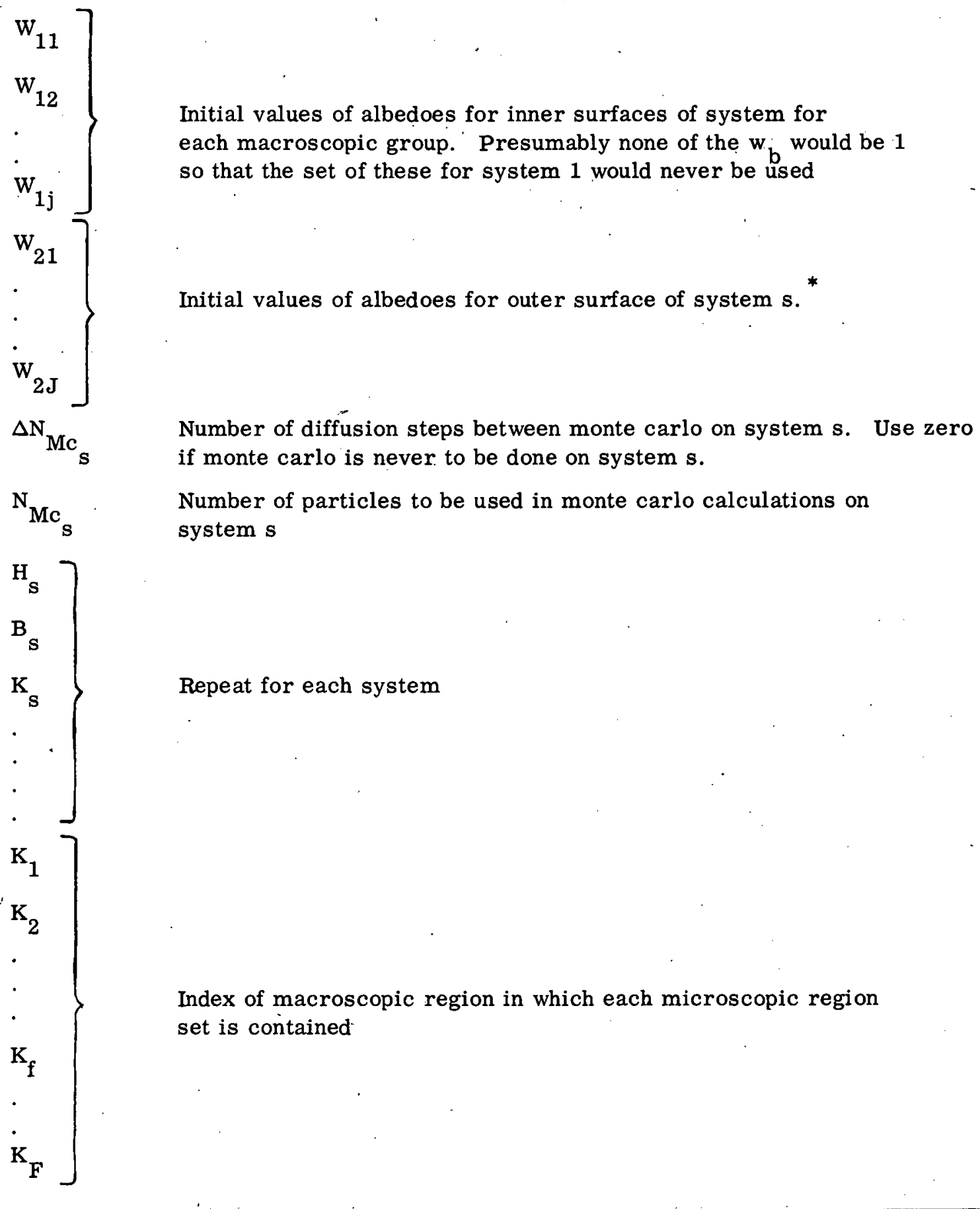

* Although the post diffusion computes albedoes for the inner and outer surfaces of each system and space is provided for them, the monte carlo only uses those indicated in its boundary data. 
C. Burnup Data

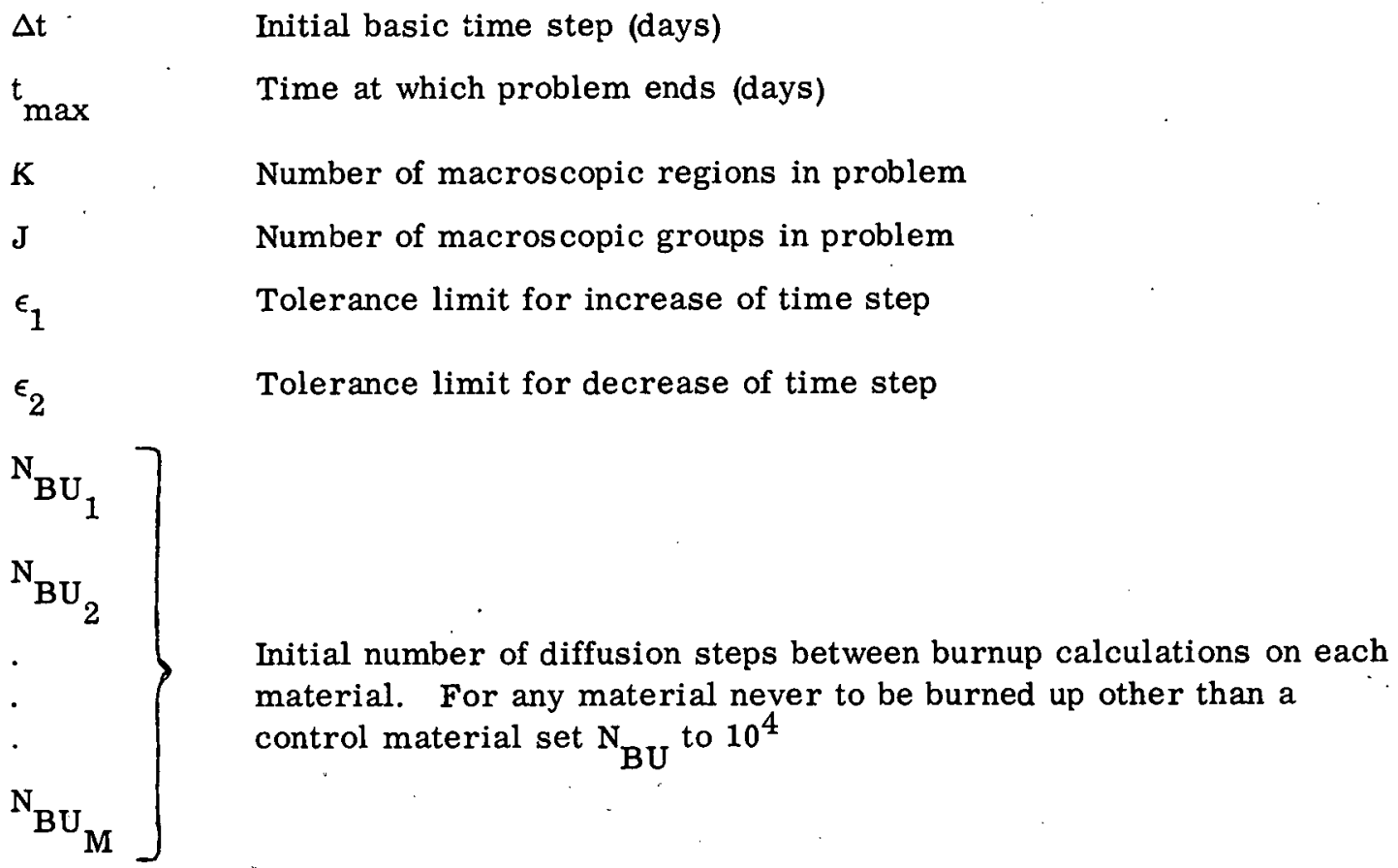

D. Diffusion Data

$\epsilon_{3}$

$\epsilon_{4}$

$\rho$

$R_{0}$

$\omega$
Tolerance for convergence of reactivity

Tolerance for convergence of source density

Geometry index for diffusion calculation

Coordinate value at first mesh point

Acceleration factor 


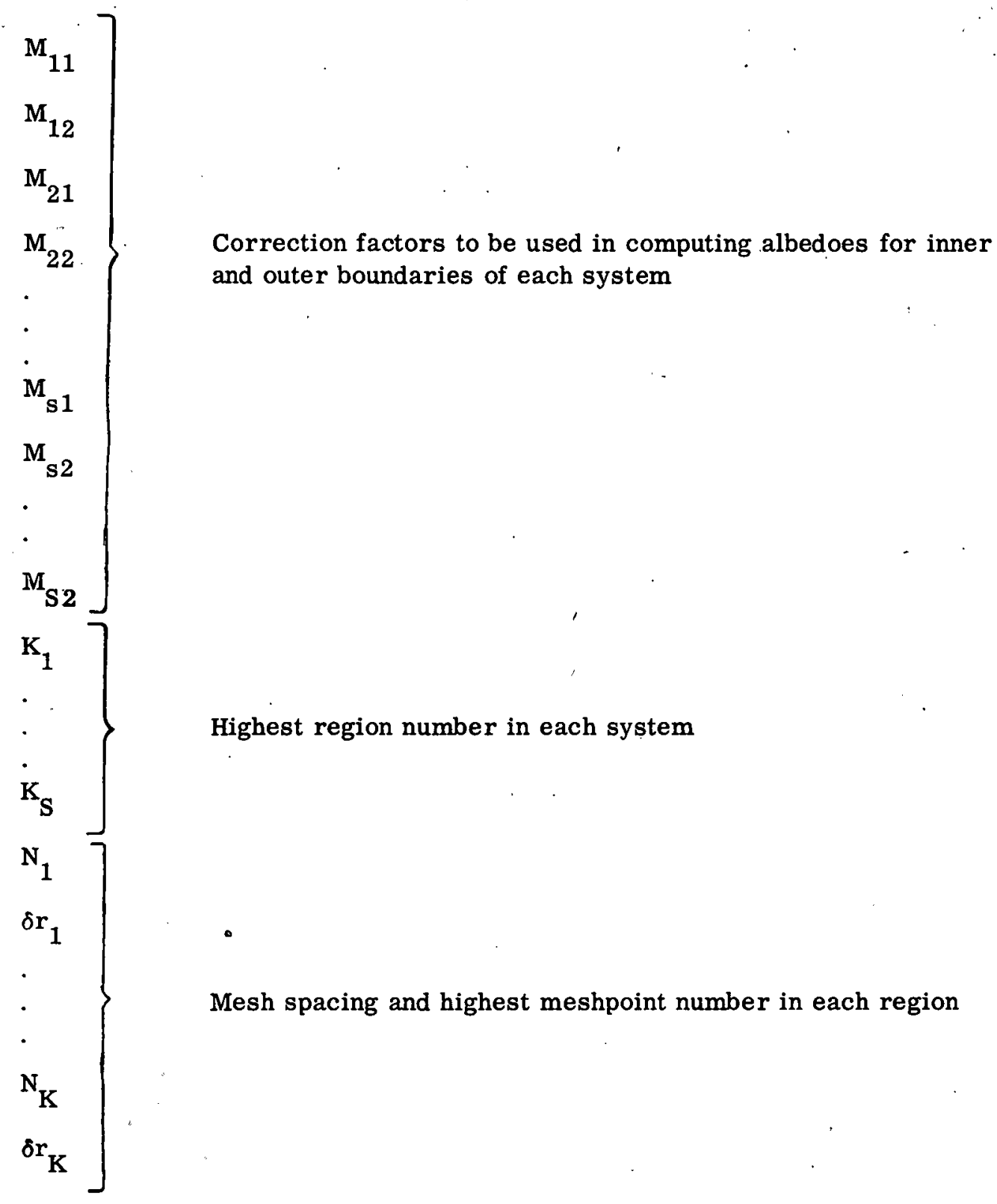


$\left.\begin{array}{l}\mathrm{B}_{1} \\ \mathrm{O}_{1} \\ \mathrm{O}_{2} \\ \cdot \\ \mathrm{B}_{\mathrm{O}_{\mathrm{J}}} \\ \mathrm{B}_{\mathrm{N}_{1}} \\ \cdot \\ \cdot \\ \mathrm{B}_{\mathrm{N}}\end{array}\right\}$

Boundary condition parameters at inner and outer boundaries

E. Criticality Data

$\epsilon_{5}$

L

$\ell_{A}$

${ }^{\mathrm{c}} \mathrm{A}$

$\left.\begin{array}{c}M_{1} \\ M_{2} \\ \vdots \\ M_{\vec{\alpha}}\end{array}\right\}$

$\mathrm{s}_{01}$

$\mathrm{s}_{02}$

.

.

Tolerance for criticality

Number of control materials

Initial active control index

Initial setting of initial control material

Control materials in order of use

System in old problem from which I. V.'s are to be taken for each system in this problem. In other words, the initial set of particles for the first monte carlo step on system $s^{\prime}$ will be taken from system $\mathrm{S}_{0 \mathrm{~S}^{\prime}}$ on the old tape 5 which is on the computer during input preparation. 
Perpendicular buckling for each region and group. Given in order of increasing $\mathrm{K}$ for those regions only which are in systems on which a monte carlo is never done. 


\section{RBU OPERATING INSTRUCTIONS}

A. Making Instruction Tape

Each RBU problem has its own instruction tape and system loader (2 binary cards which load the instruction tape). This tape is made as follows:

1. Ready Tapes

$\begin{array}{lc}\text { A1 } & \text { Sos system } \\ \text { A2 } & \text { Scratch } \\ \text { B1 } & " \\ \text { B2 } & " \\ \text { B6 } & "\end{array}$

2. Sense Switches

1 down

2-6 up

3. Ready the RBU squoze decks in the card reader, in the order in which they appear on the layout of tape 1. Each squoze deck must be preceded and followed by the necessary scat control cards, as follows:

JoB

LG

Squoze deck (including any modifications)

Blank card

Go

4. Clear

5. Load tape

After the last record of the instruction tape (the input routine) has been written, the loader cards will be punched and the machine will stop on the following instruction:

77777 HTR 0

The instructions will be on tape B6. A2 may be printed, if desired, to read any comments by Modify and Load. 
B. Translating Input (Input Routine) .

Problem input is read from cards or tape, translated, and written on the instruction tape (starting with file 3 ).

1. Ready tapes (see description of input routine).

2. Sense switches

1 down

$2-6$ up

3. Ready the following cards in the card reader:

RBU system loader ( 2 binary cards)

JOB

LG

RBU squoze loader deck

Blank card

GO

4. Clear

5. Load cards

During the execution of the input routine, two cards will be punched:

a. Tape position card

b. Restart card

These will be used to initiate execution of the problem.

Final stop (see Input Routine).

C. Problem Execution and Restart (without changes)

The procedures for beginning execution of a problem and continuing a problem which has been interrupted are the same. The start routine loads the RBU monitor into memory locations 0 through ORIGIN-1, copies the problem data (beginning in file three of the instruction tape) onto RBU tapes 2-9, selects the desired starting point (from the restart card) and begins execution of the problem. Operating procedure is as follows:

* To be published shortly by Hanford Laboratories Operation. 
1. Ready tapes

$\begin{array}{ll}\text { A6 } & \text { scratch } \\ \text { A7 } & " \\ \text { A8 } & " \\ \text { A9 } & " \\ \text { A10 " } \\ \text { B6 } & \text { RBU inst. tape } \\ \text { B7 } & \text { Scratch } \\ \text { B8 v } \\ \text { B9 " }\end{array}$

B10 Scratch (or previous out-put tape, if this is a restart)

2. Sense switches

$1-\dot{6}$ up

3. Ready the following cards in the card reader:
a. RBU system loader ( 2 cards)
b. Tape position card
c. Restart card

4. Clear

5. Load cards

The problem is now started and may be followed on the on-line printer. If the operator wishes to take the problem off the machine, he may put s. sw. 6 down. When the problem reaches a convenient stopping pointo dump and restart preparation will be automatically initiated.

D. Restart with Changes

It is possible to insert changes in the data which are transcribed from tape $B 6$ to the main problem RBU tapes at restart time. These changes are made between the time the data are read from B6 and the time they are written on the other tapes; therefore they will not alter the contents of $\mathrm{B} 6$. The format of change input is specified in another section of this manual.

* This may occur some time after the switch is set, particularly in the case that the monte carlo code is in operation at the time the switch is set. See overall flow chart. 
The procedure for restarting a problem with changes are as follows:

1. Ready tapes

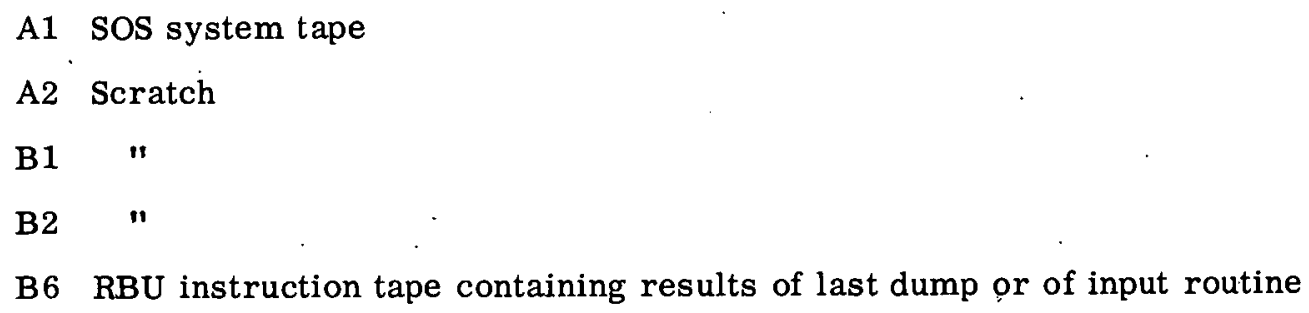

2. Sense switches

1 down

2 down

3-6 up

3. Ready cards in card reader:
a. RBU system loader ( 2 cards)
b. JOB
c. LG
d. RBU squoze loader deck
e. Blank card
f. DS1 (SOS control card for data-sentence input)
g. Change input in data-sentence form
h. Blank card
i. GO
j. Tape position card from dump or input routine
k. Restart card from dump or input routine

4. Clear

5. Load cards

When SOS has finished its job of translating the change input, the machine will stop. At this point, the SOS tape (A1, A2, B1, B2) are no longer needed and may be removed, if desired, to free the tape units for the following additional tapes:

\section{A6-A10 Scratch}

B7-B9 "

B10 Previous output tape 
After the above tapes are ready, press start and the problem will be executed.

E. RBU MONITOR

The RBU monitor remains in memory locations 0 through ORIGIN-1 throughout the run of a problem. All communication between the monitor and other RBU programs is $r$ eferenced to location ORIGIN, as indicated in the calling sequences below. The 5 functions of the monitor are:

1. Loading and positioning the instruction tape.

2. Dump and restart preparation.

3. On-line output.

4. Automatic punching of tape-position and restart cards.

5. Error analysis.

These are described in the following paragraphs:

1. Loading and Positioning the Instruction Tape

Calling sequence:

STL

ORIGIN-21

TXL

ORIGIN-20, $0, \mathrm{R}$

PZE

$\mathrm{T}, 0, \mathrm{~S}$

RETURN (if $S \neq 0$ )

$R$ is coded as an instruction tape record number (see tape 1 layout).

$T$ is coded as a machine address. If $T=0$, transfer after loading record $R$ is made to the location specified in the third word in record $R$ (see instruction tape record ID). If $T \neq 0$, transfer after loading record $R$ is made to location $T$. $S$ is coded as a zero or 1 . If $S=1$, record $R$ is not loaded, but the instruction tape is positioned to the end of record $R$ and control is transferred to the RETURN location of the calling sequence ( $T$ is ignored). If $S=0$, record $R$ is loaded and transfer is made according to what is coded for $T$.

2. Dump and Restart Preparation

Calling sequence:

STL

ORIGIN-21

TXL

ORIGIN-19, $0, \mathrm{~A}$ 
$A$ is coded as a machine address. The core dump record (record \#202) will contain $A$ in the address of its third word. The restart card will indicate record \#202 and loc. $A$ as the restart point.

Manual dump is obtained by a manual transfer to $16_{\varepsilon}$

3. On Line Output

The monitor contains an on-line print routine, which is reached by the following sequence:

TSX ORIGIN-23, 4

Print calling This routine uses a "squoze" calling sequence to indicate seq. , variable data to be printed and format.

no. of wds.

RETURN

4. Punching Tape - Position and Restart Cards

Calling sequence:

STL ORIGIN-21

TRA ORIGIN-22

PZE L, , R

RETURN

$\mathbf{R}=$ restart record number

$\mathrm{L}=$ restart location

This causes punching of tape-position and restart cards, without the dump procedure, and return to location RETURN. This is primarily for use by the monte-carlo program, which causes these two cards to be punched before it begins computing on each system. If an error or machine trouble occurs, the cards provide a restart point.

5. Error Analysis

a. Non Floating Point Errors

In each RBU program, when an error is sensed by the program, the instruction:

STR Y, T, D is executed.

The $\mathrm{D}$ field contains the location to which control may be returned after analysis and correction of (or ignoring) the error. $D=0$ means no recovery. 
The $\mathrm{T}$ field tells the monitor whether to use a standard error routine within the monitor $(T \neq 0)$ or call in a special error program $(T=0)$.

At present, there are only two standard error routines in the monitor: $T=1$ means redundancy check failure. In this case, $Y$ must be the address of the tapeselect instruction which caused the failure. $T=2$ means end-of-tape, with the tape address in $\mathrm{Y}$.

If $\mathrm{T}=0, \mathrm{Y}$ is taken to be the location within a special error routine where. this error is analyzed.

As soon as the STR instruction is executed, the monitor will print the location of the STR instruction +1 (in octal). In any of the following situations:

$$
\begin{aligned}
& \mathrm{T}=3-7 \\
& \mathrm{~T}=0 \text { and } \mathrm{Y}=0 \\
& \mathrm{~T}=0, \mathrm{Y} \neq 0
\end{aligned}
$$

but the program in memory does not have a special error routine, the monitor will be unable to make any further analysis, and will print a message to that effect. In all other cases, instructions to the operator, and further explanation of the error will be printed.

The machine will stop before returning to the program, allowing the operator to dump or return (to the location specified in $\mathrm{D}$ ).

\section{b. Floating Point Errors}

The 709 floating point trap is used to detect $A C$ and $M Q$ overflow or underflow during floating point operations. Following such an error, the monitor will print the location +1 of the instruction which caused the error, and what the error is. If the e rror is considered to be insignificant, control is automatically returned to the program. If not, the monitor proceeds as though the instruction STR Z, O, D had been executed. Where $\mathrm{Z}$ is taken to be the location in a special error routine of a floating point error code, and $D$ is equal to the location which was printed when the error was detected. 
F. Tape Position and Restart Cards

1. Tape Position Card

9-L Word Sequence number

9-R Word. Checksum

$\left.\begin{array}{l}\text { 8-L } \\ \text { O-R }\end{array}\right\} \begin{aligned} & \text { Tape contents and positions (see } \\ & \text { description of tape position } \\ & \text { block) }\end{aligned}$

$12 \mathrm{~K}\}$ Not used

2. Restart Card

9-L Word Sequence number

9-R Word Checksum

8-L Word Record number 7 For restart

8-R Word Location $\}$

$\left.\begin{array}{ll}7-\mathrm{L} \text { Word } & \begin{array}{l}\text { Record number } \\ \text { Location }\end{array}\end{array}\right\} \quad$ From dump (ignored by restart routine)

$\left.\begin{array}{l}6-\mathrm{L} \\ 12-\mathrm{K}\end{array}\right\} \quad$ Not used

These cards are always punched and used in pairs. Both cards of any pair used must have the same sequence number. The sequence number for the first set punched in any problem (these are punched by the input routine) is one. The sequence number is increased by one each time a pair of these cards is punched.

The 7-L and 7-R words of the restart card are not interpreted by the restart routine; but are included on the card to tell the operator where the instructions which caused the card to be punched were located. The 8-L and 8-R words determine where computing will begin on re-start. These may be punched by hand, to begin at any desired point. 


\section{G. Input Change Cards}

The input changes are made in the SCAT Data Sentence format wi th the first word of the change data stored in memory location $(4700) 10$. The data required is indicated in the following list.

RBU tape number, that is the tape on which the change is to be made.

Number of the file in which the change is to be made.

Record number in the file which is to be changed.

Word number in the record of the first word to be deleted.

Word number in the record of the last word to be deleted.

Word (if any) to replace first deleted word.

Word (if any) to replace second deleted word.

.

$\cdot$

Last word to be inserted (the number of words inserted may be less than, equal to, or more than the number of words deleted).

-0 (tag indicating last word to be inserted).

RBU tape number

.

Repeat for each set of changes

.

-0 (additional tag indicating last set of changes).

\$ (data Sentence Program tag indicating end of block).

The changes may be given in any order that is convenient. Normally they will be all in one sentence. If for some reason it is desired that more than one sentence be used, the Data Sentence Program requires that the absolute location of the first word of each sentence be given in the normal way. This is, of course, easily computed by adding 4699 to the member of preceding change words. 
The input change program does not provide for the deletion or insertion of complete records so that any changes made by means of it must be such as to maintain a one-toone correspondence between the recorder of the old and new problems.

\section{H. Tape Position Block}

The Tape Position Block consists of two words for each of tapes 2 through 10. For each tape, the first word describes the tape contents, the second the tape position. Define

$\mathrm{R} \equiv$ number of records following the last end of file mark on the tape

$\mathrm{F} \equiv$ number of end of file marks on the tape

$\mathbf{R}^{\prime} \equiv$ number of records between the last end of file mark which has already been read and the present position of the read head

$F^{\prime} \equiv$ number of end of file marks between the load point and the present position of the read head

Then the tape position block is as follows

$$
\begin{aligned}
& (\mathrm{R}, \mathrm{F})_{2} \\
& \left(\mathrm{R}^{\prime}, \mathrm{F}^{\prime}\right)_{2} \\
& \left(\mathrm{R}, \mathrm{F}_{3}\right. \\
& \left(\mathrm{R}^{\prime}, \mathrm{F}^{\prime}\right)_{3} \\
& \cdot \\
& \cdot \\
& \left(\mathrm{R}^{\prime},, \mathrm{F}^{\prime}\right)_{10}
\end{aligned}
$$


XVIII. RBU FLOW CHARTS 


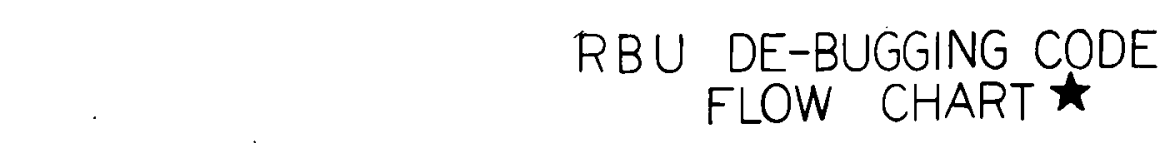

START
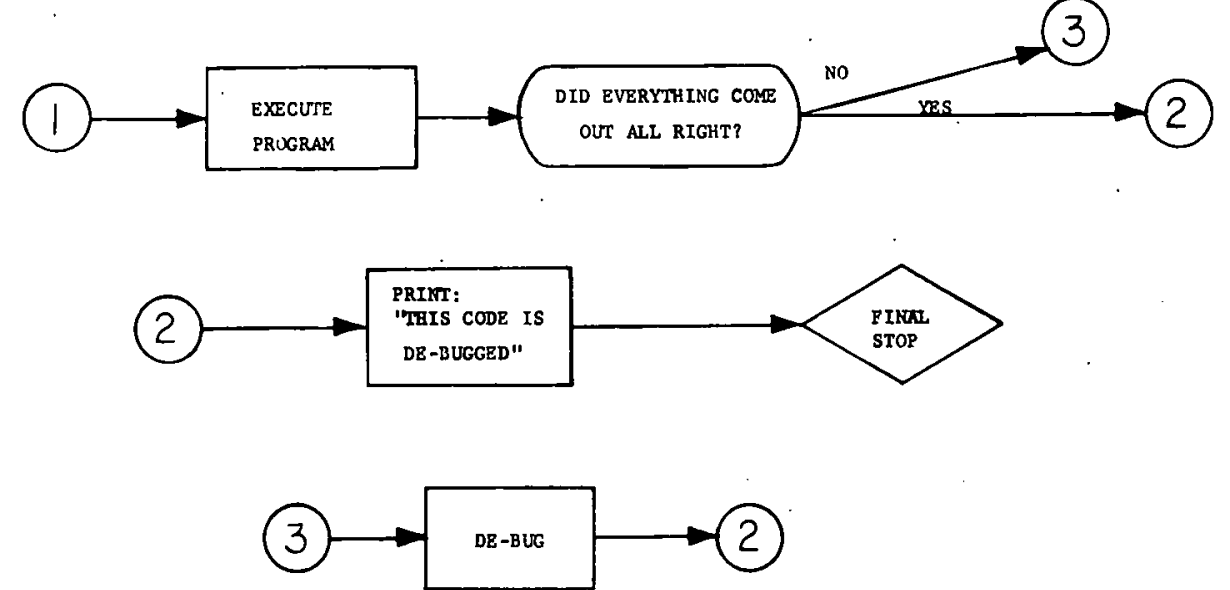

A programing of thls portion of RBU 18 not yet complete due to certaln obvlous but minor problems.

Suggeations from the readers of thie raport will be menuslastically welcomed. 

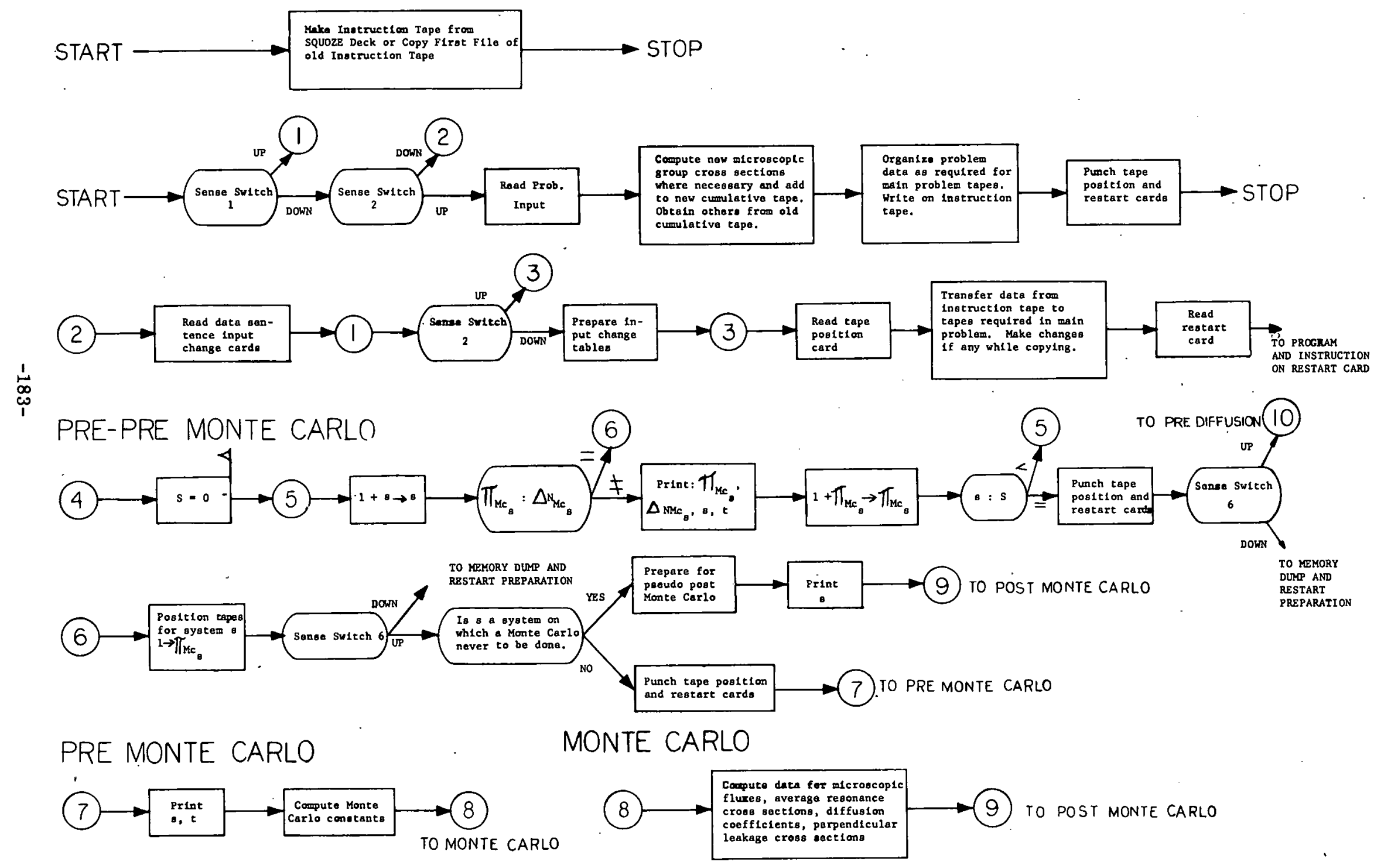
POST MONTE CARLO

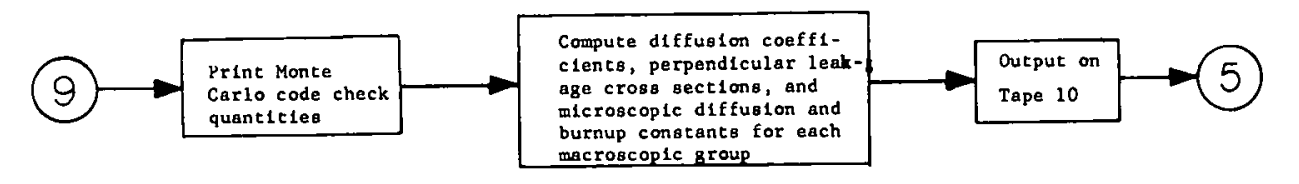

PRE DIFFUSION

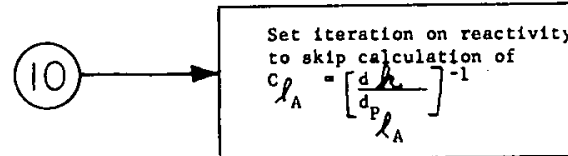

DIFFUSION

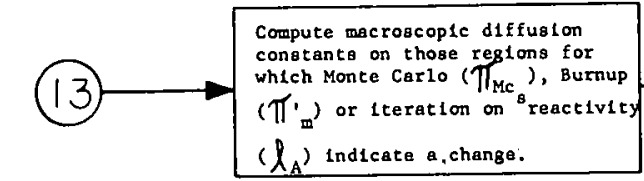

$\left(l_{A}\right)$ Indicate a,change.

TO DIFFUSION

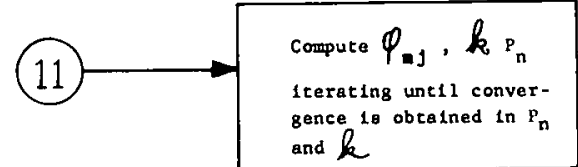

TO ITERATION ON REACTIVITY
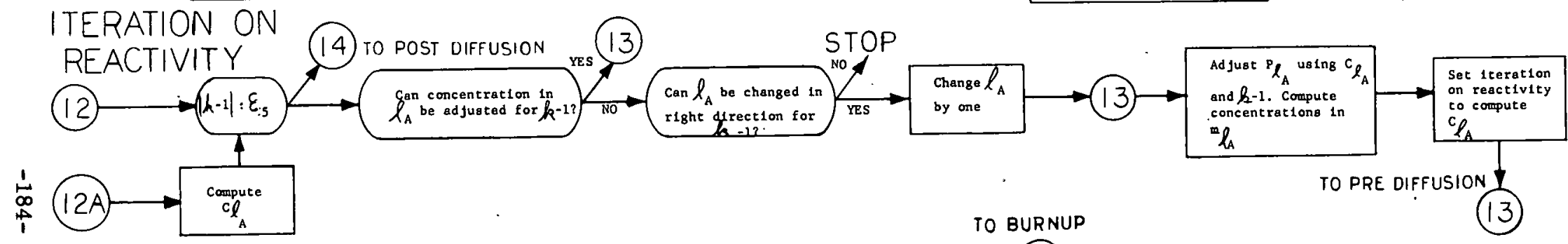

TO PRE DIFFUSION

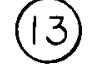

\section{POST DIFFUSION}
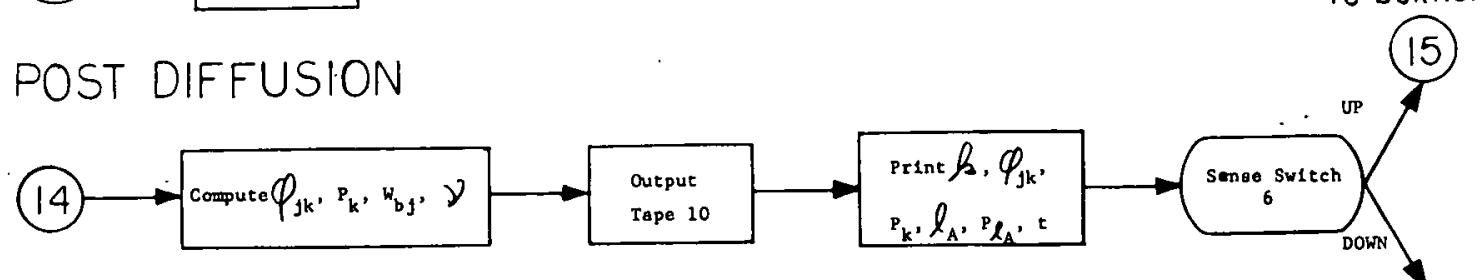

(15)

BURNUP

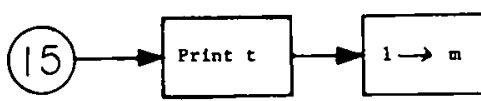

TO MEMORY DUMP.

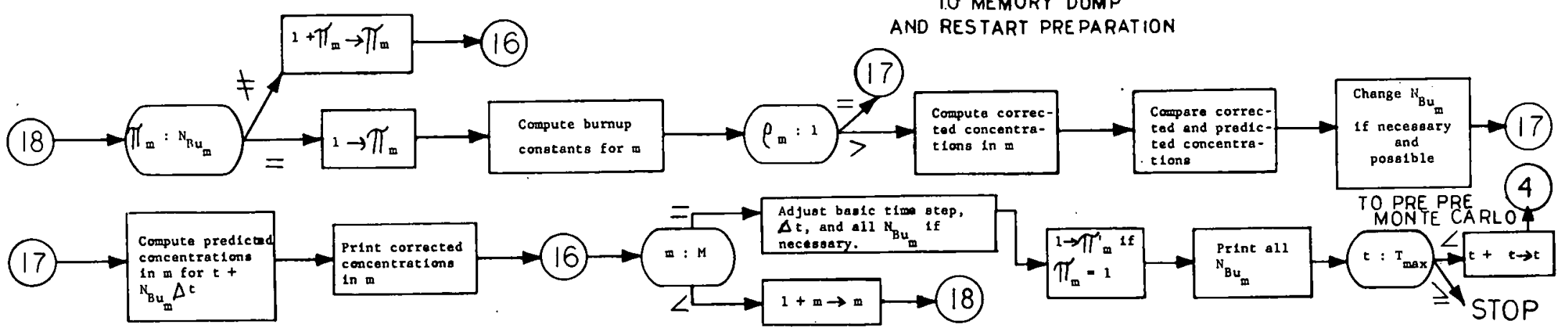




\section{CONDENSED MONTE CARLO} FLOW CHART

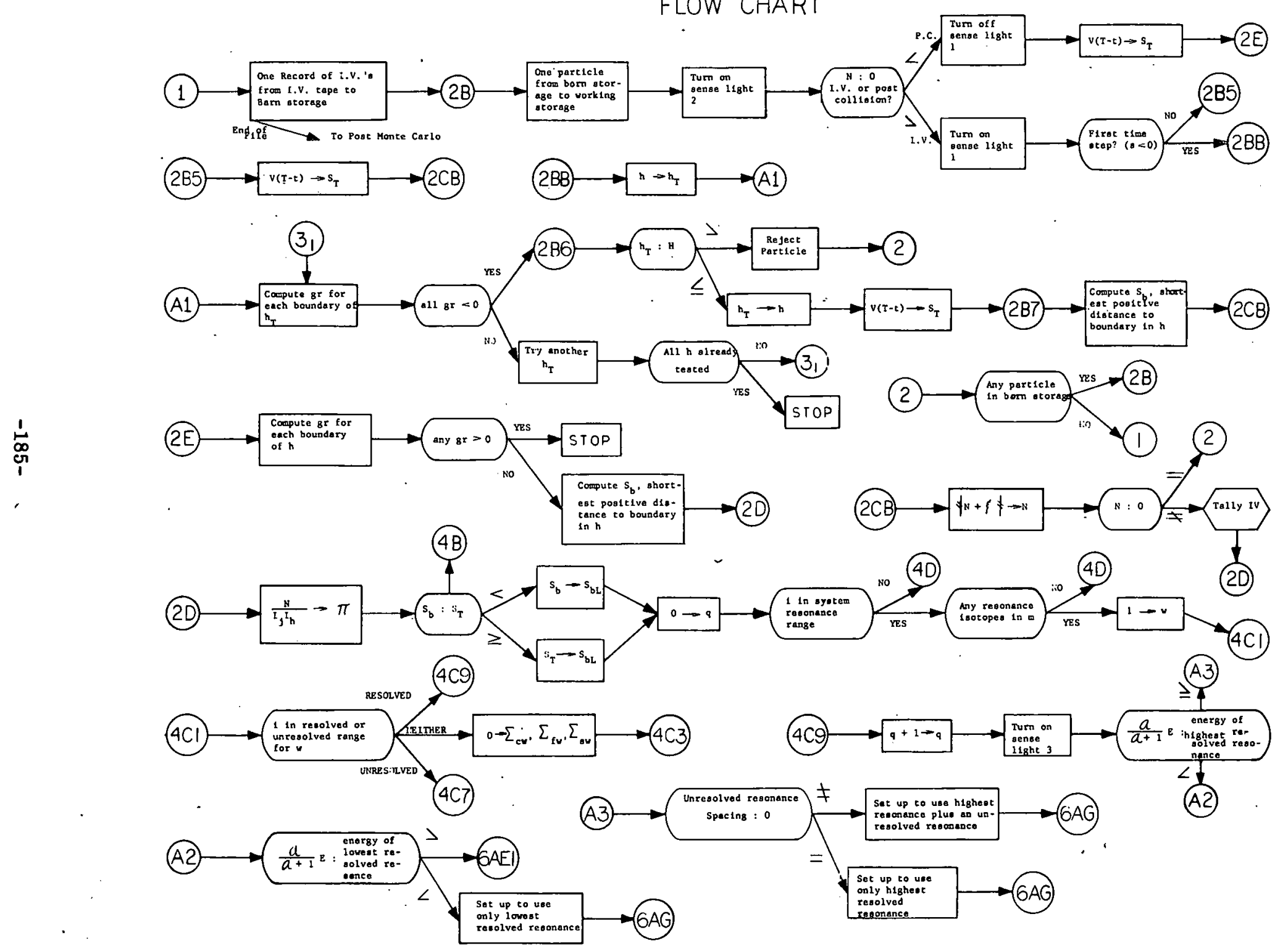




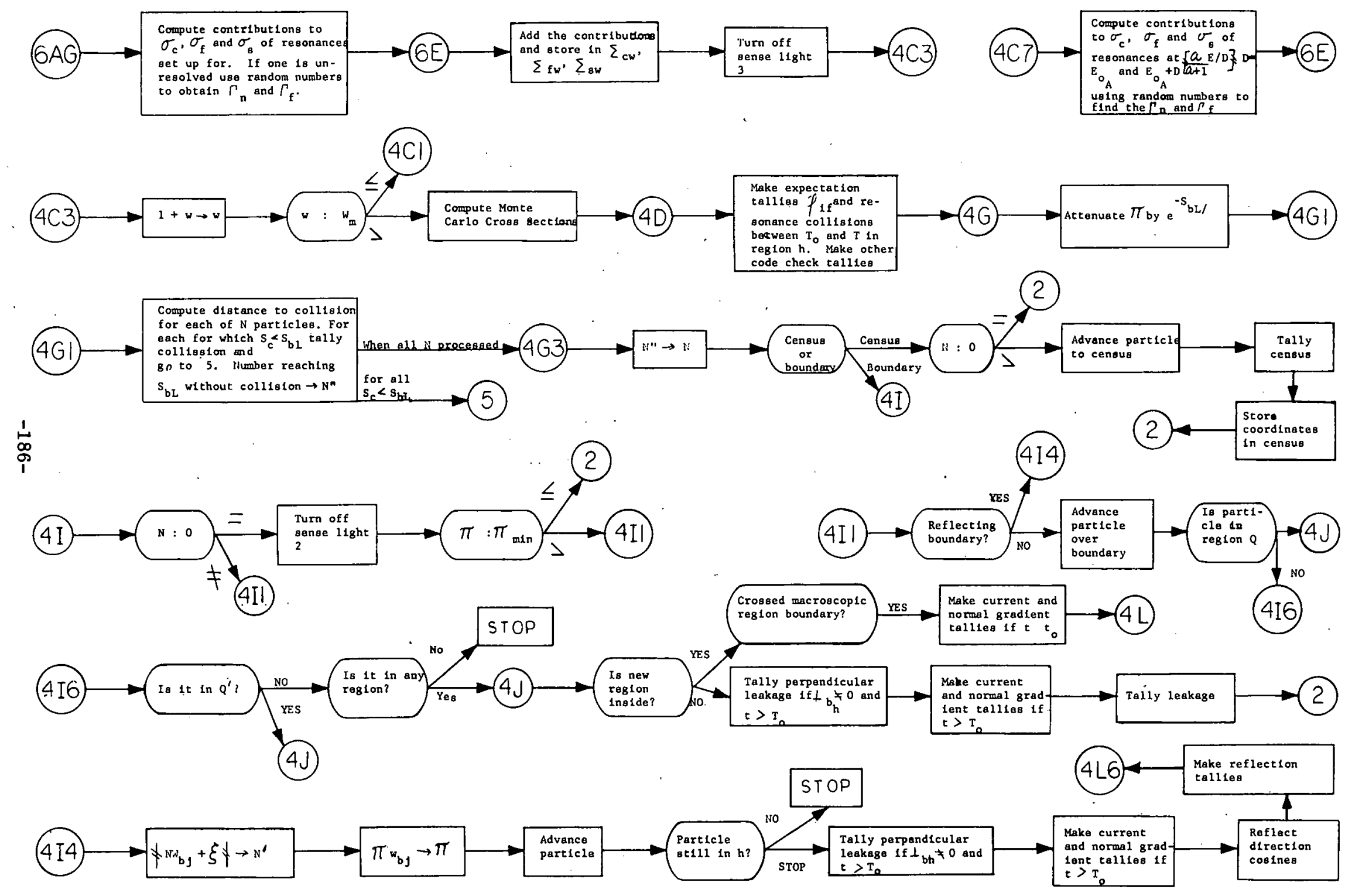

i 


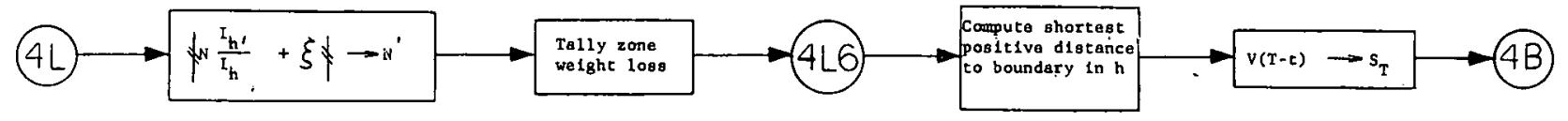

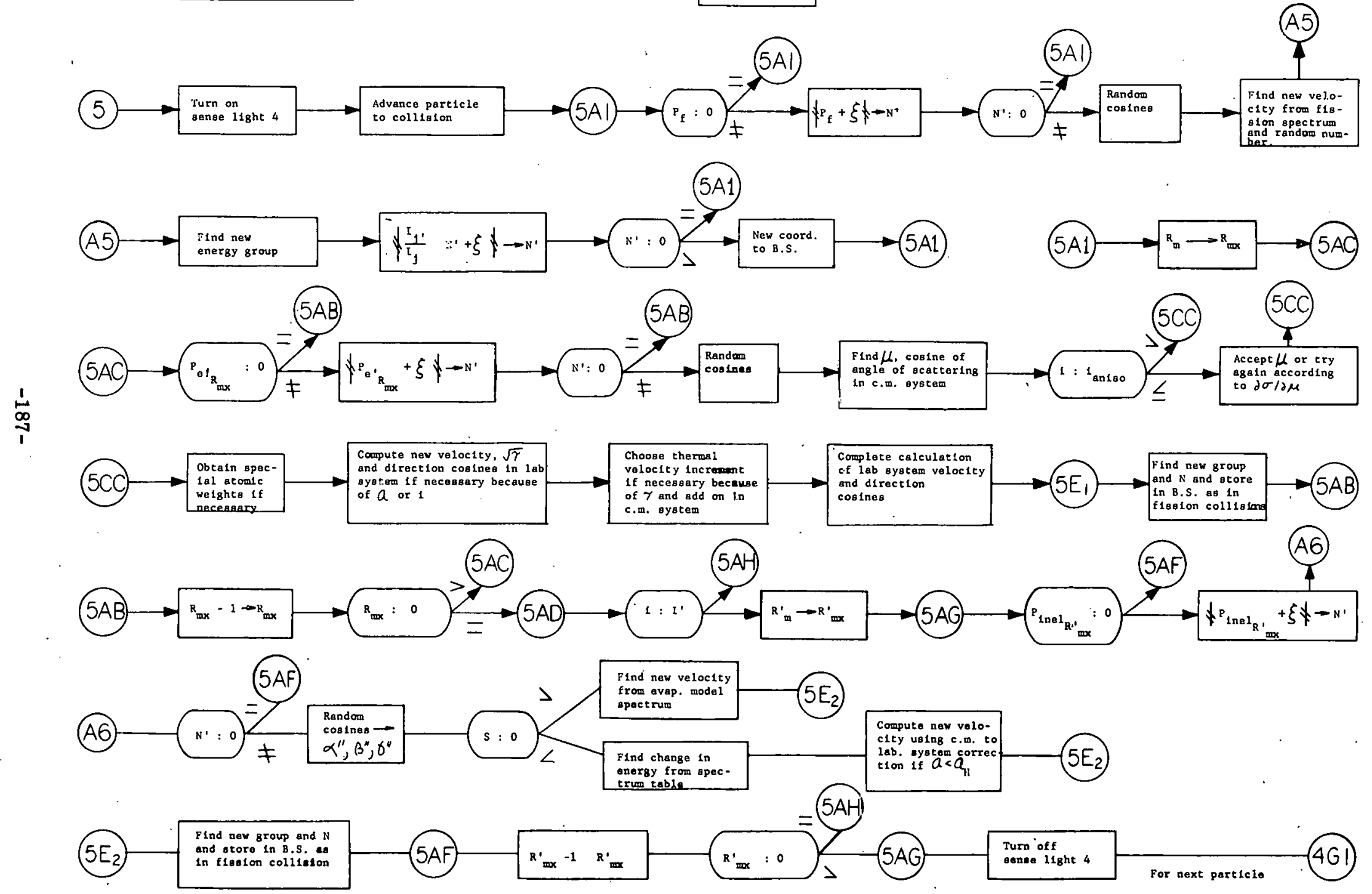




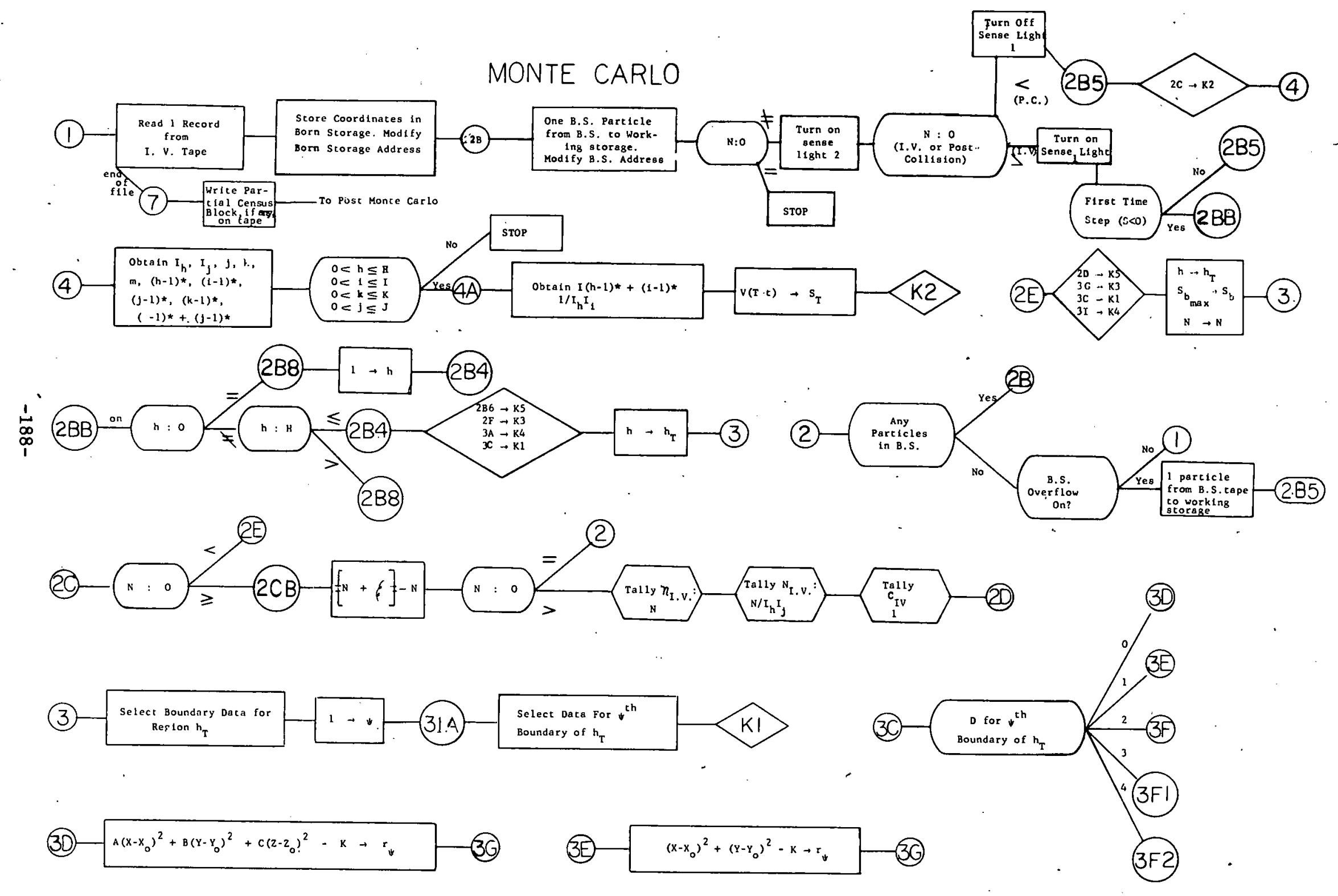




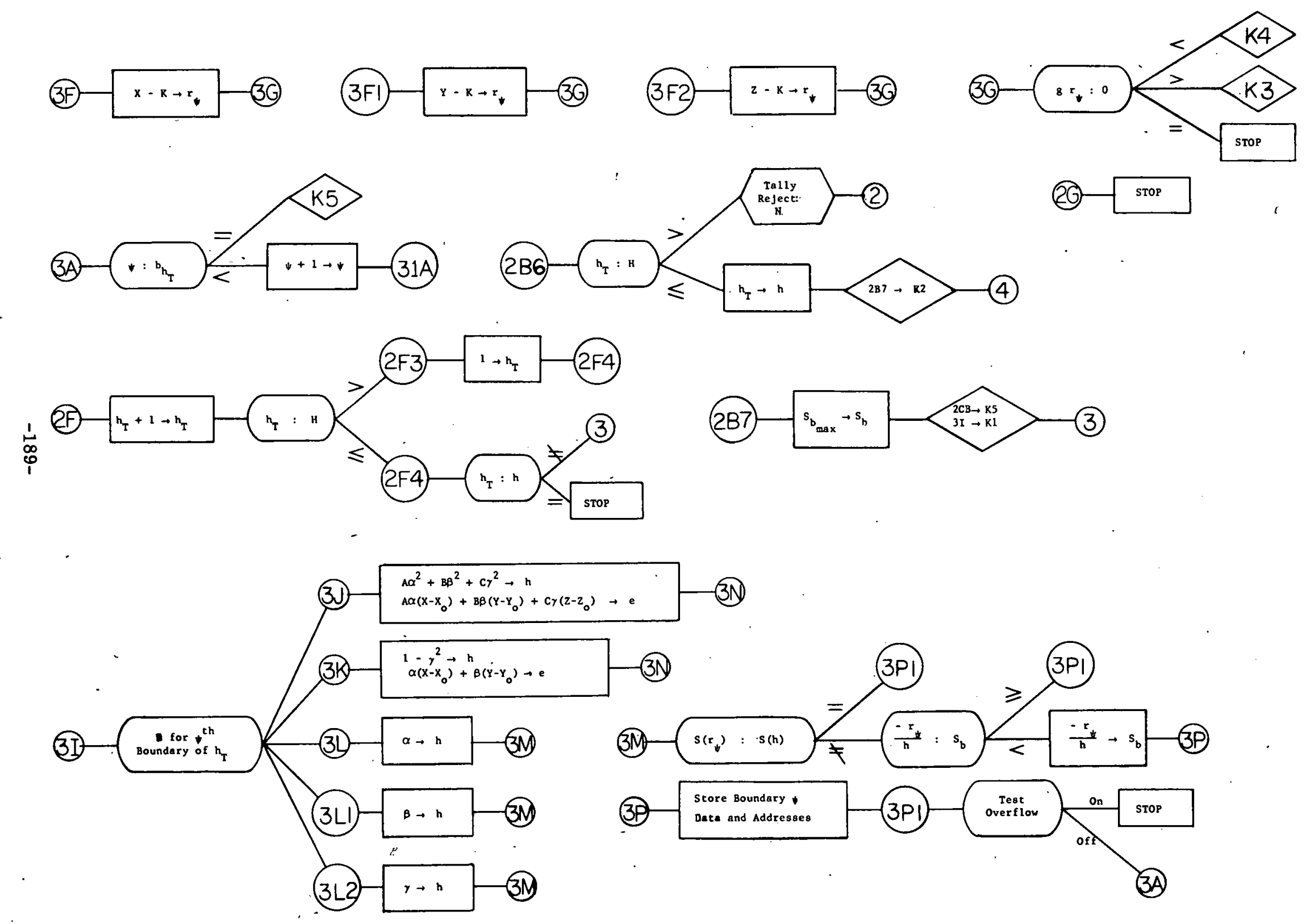



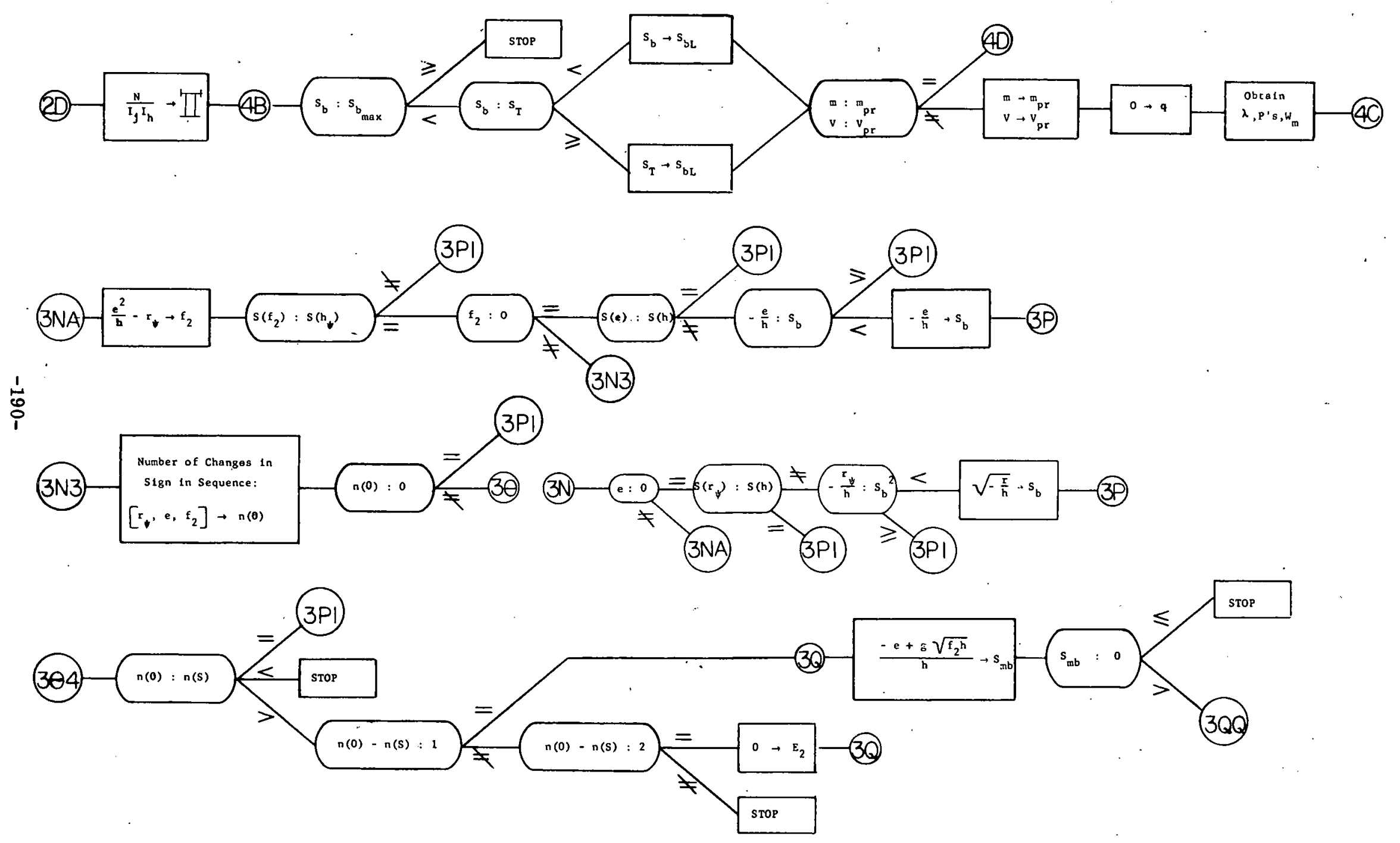


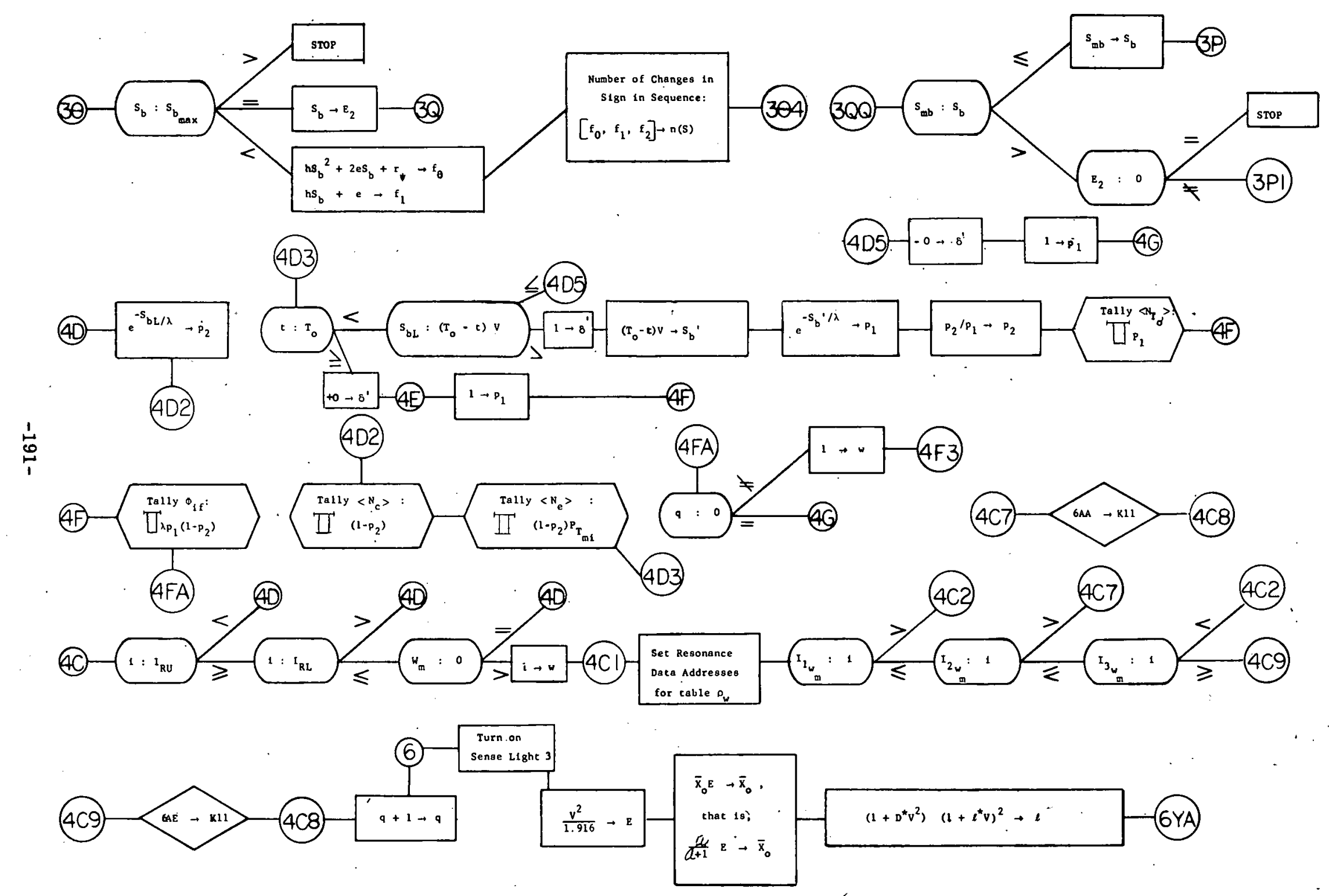




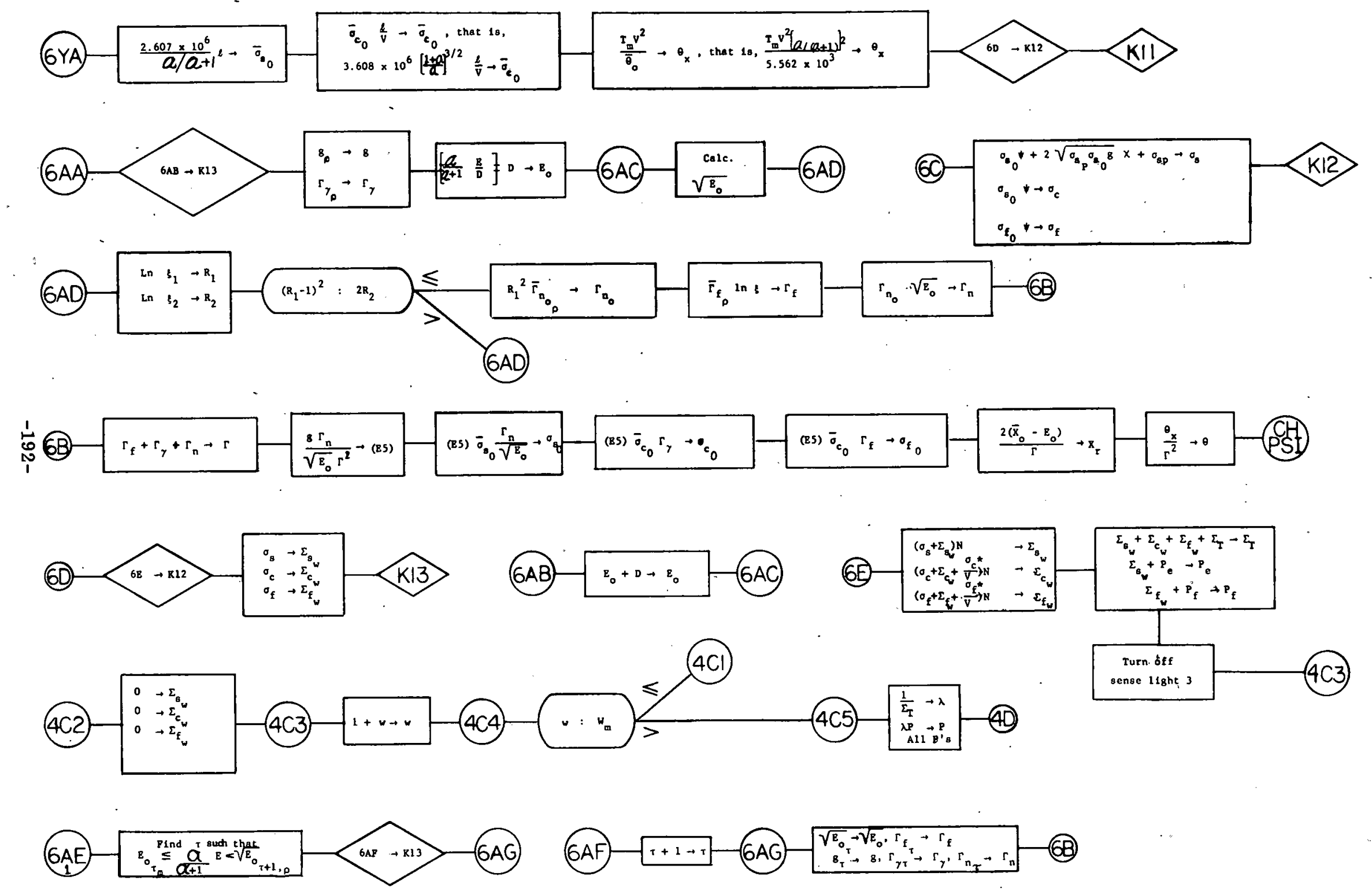




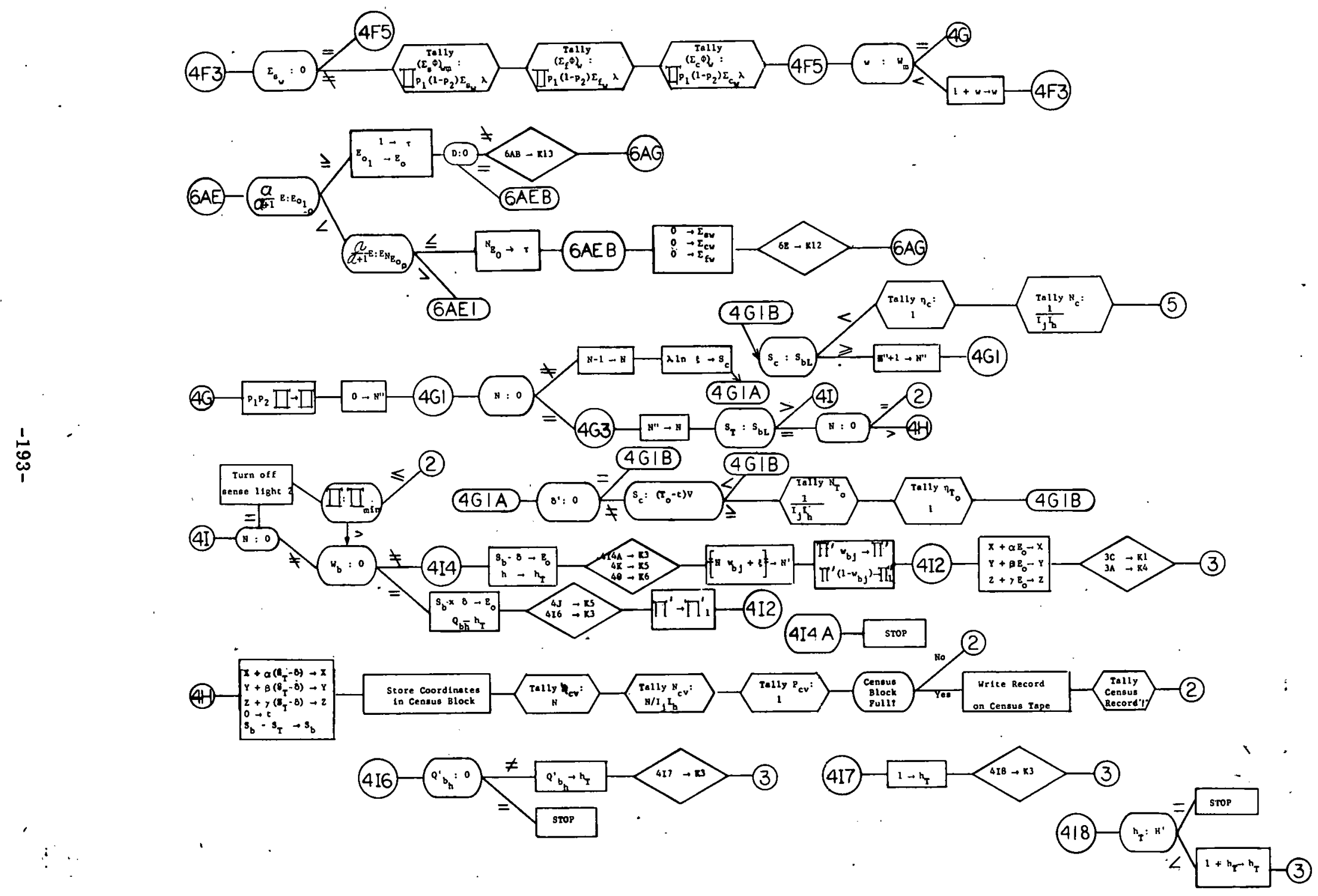




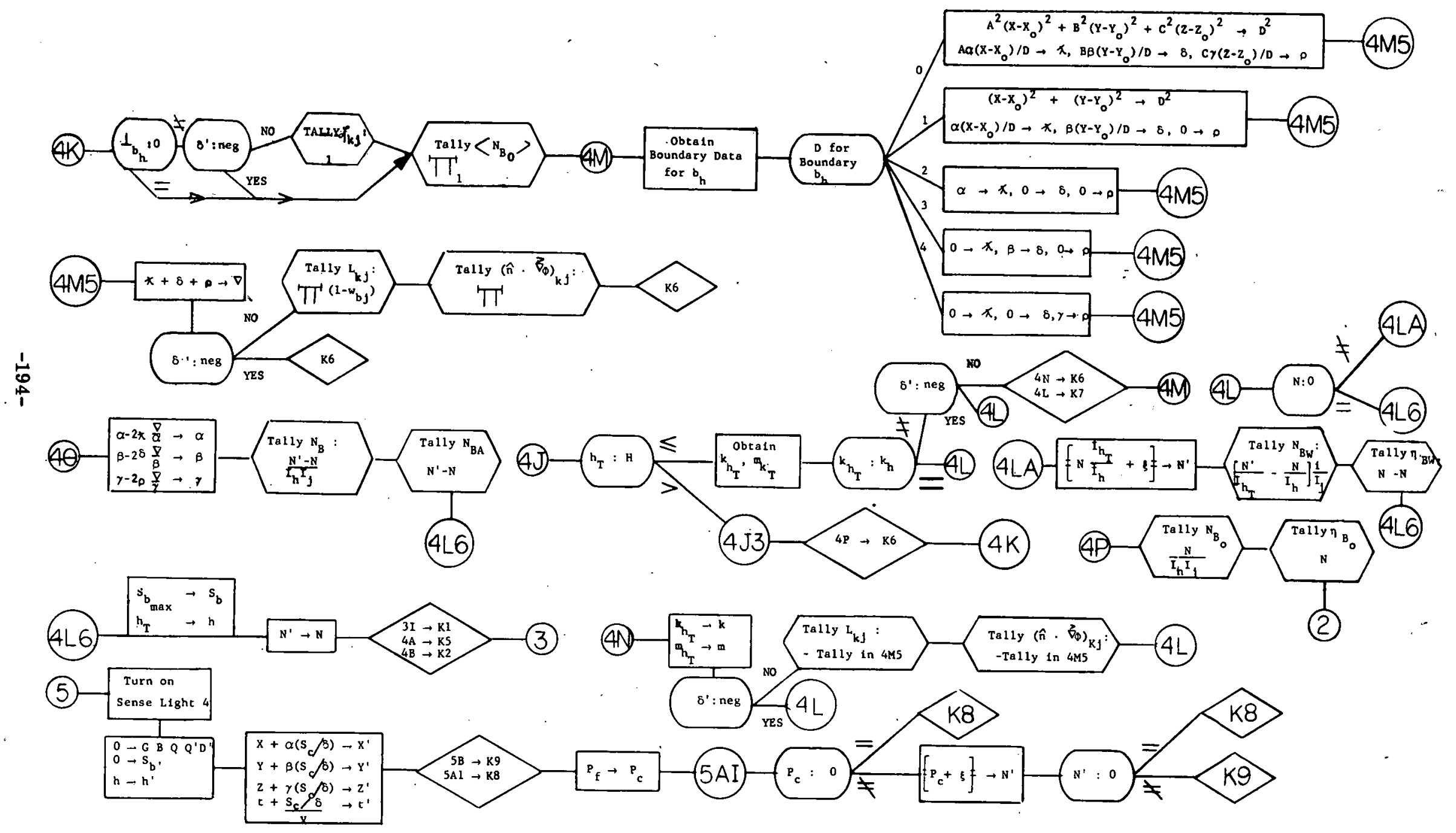




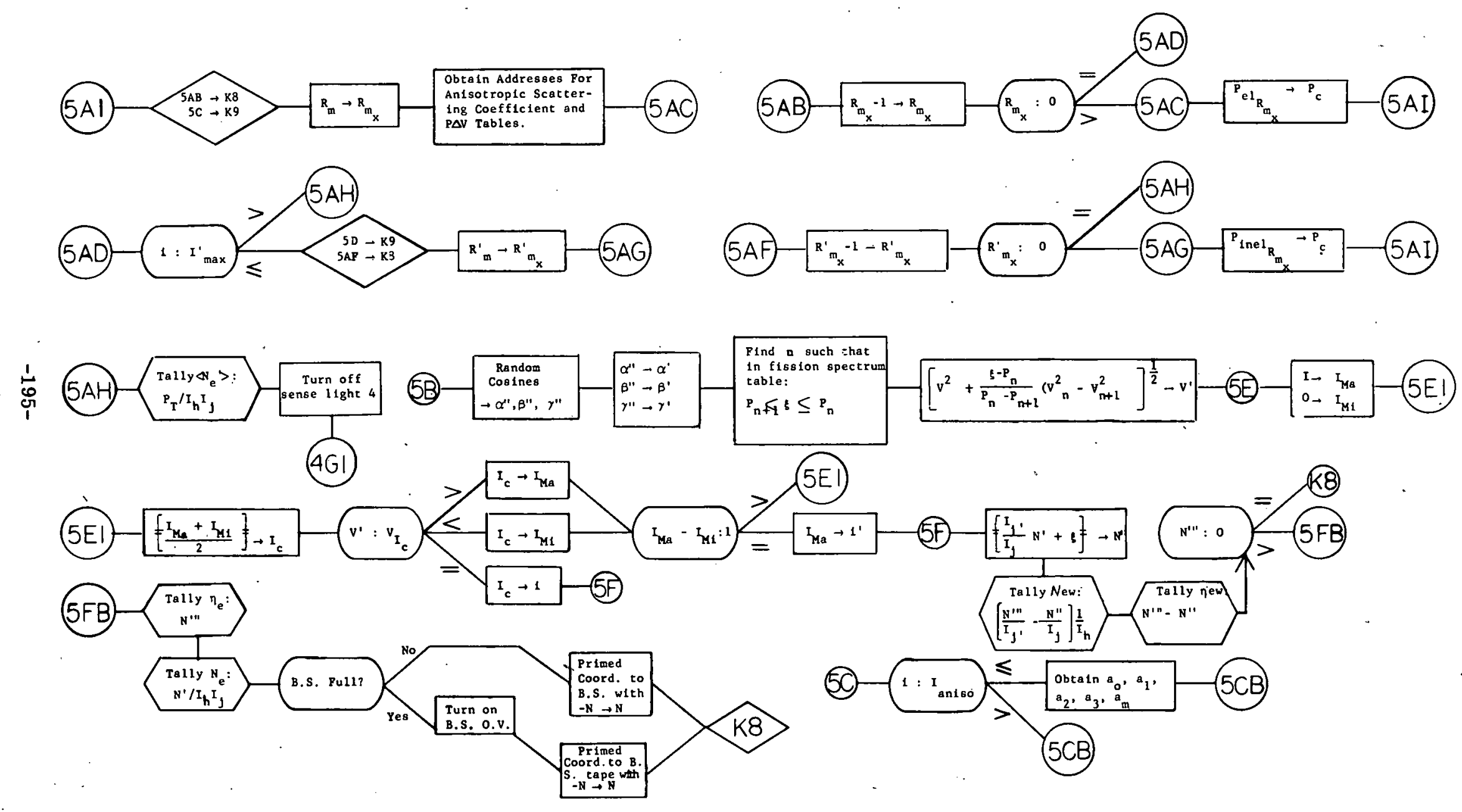




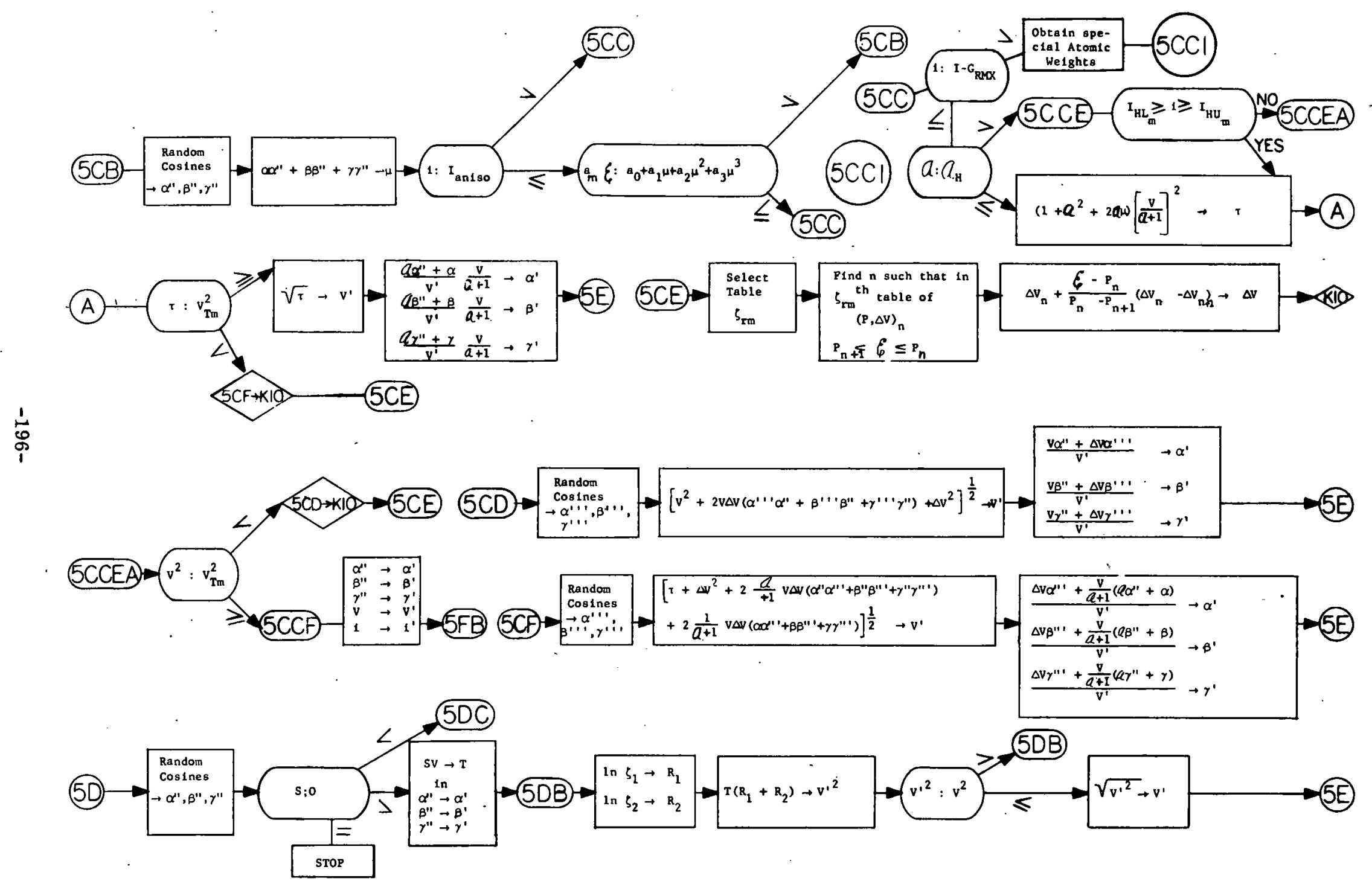



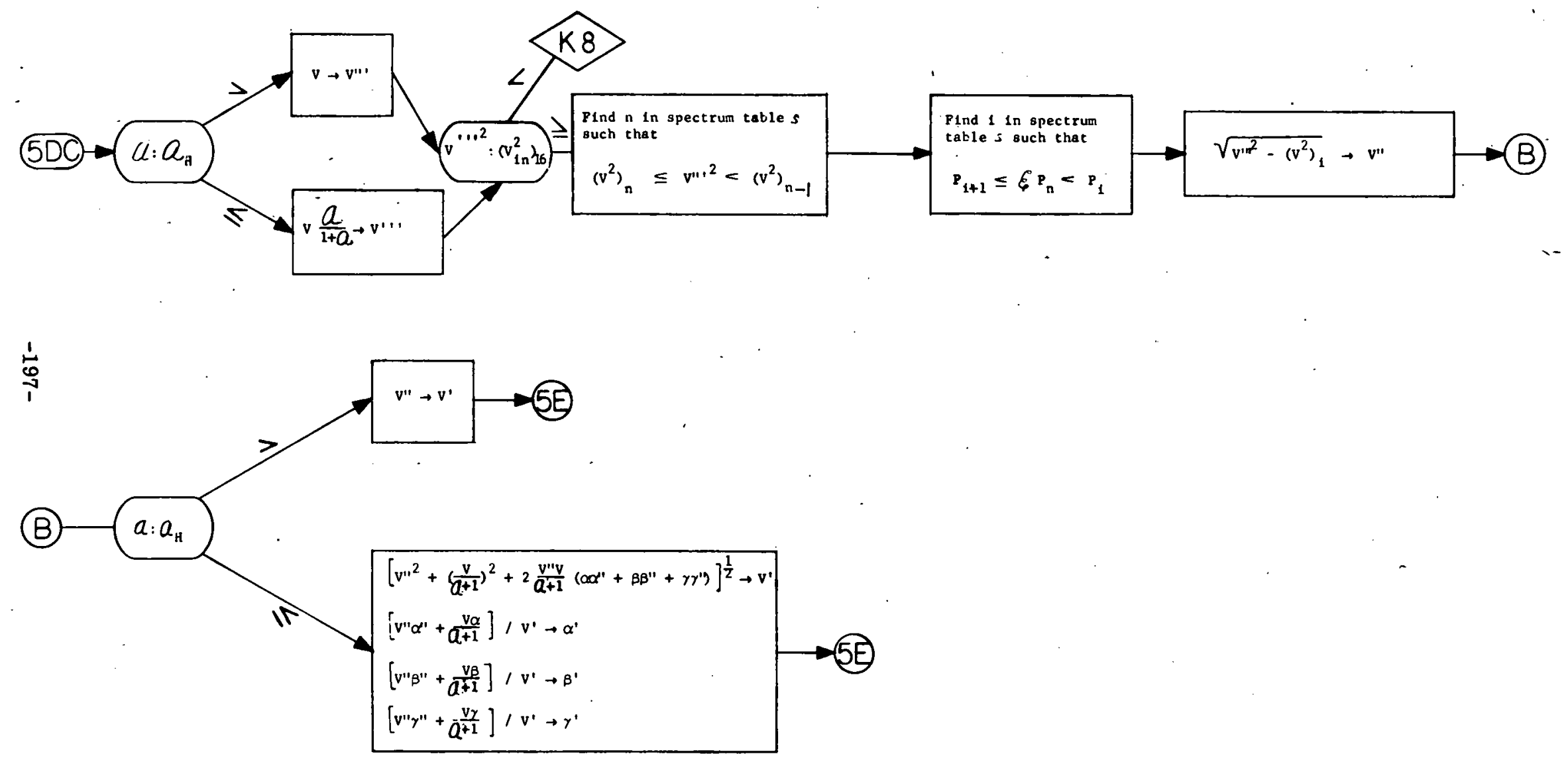

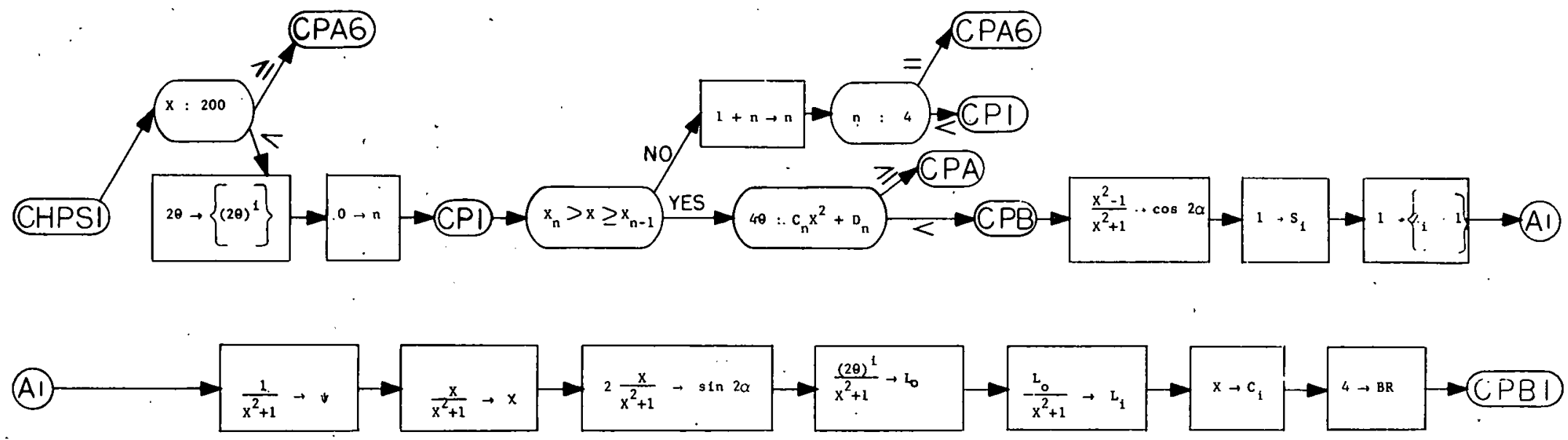

1
$\stackrel{\circ}{0}$
0
1

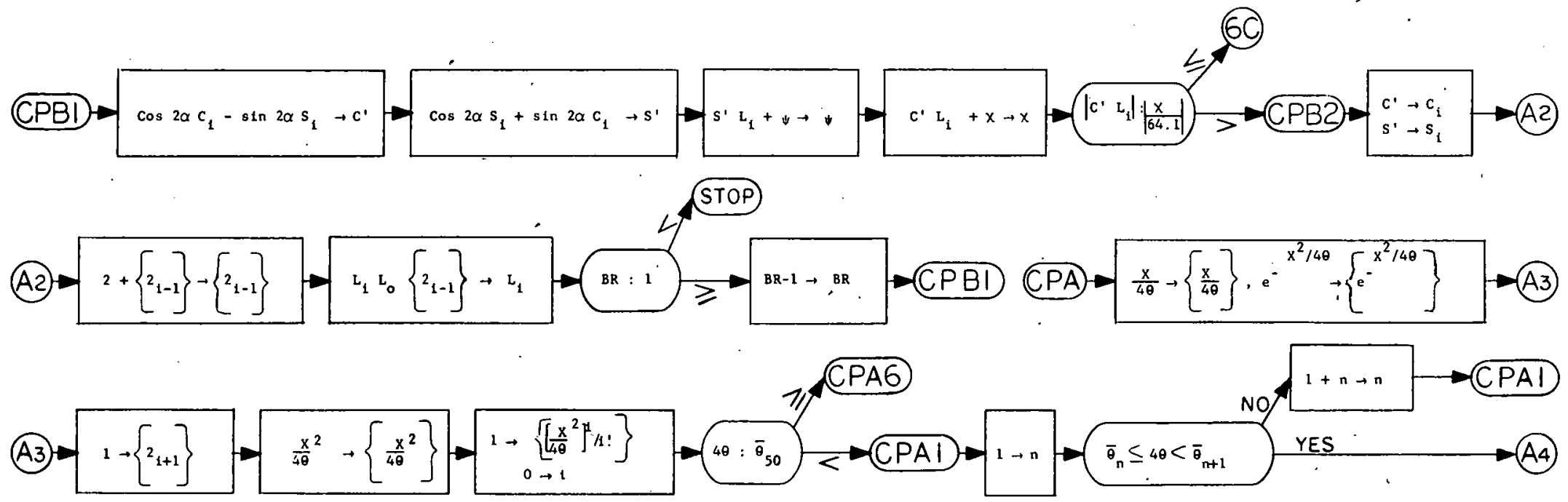

: 


$$
\text { (A4) } \Rightarrow \bar{r}_{n}+\frac{4 \theta-\bar{\theta}_{n}}{\bar{\theta}_{n+1}-\bar{\theta}_{n}}\left(\bar{r}_{n+1}-\bar{r}_{n}\right)-\bar{o}_{1} \rightarrow \psi_{1} \rightarrow \frac{v_{4}}{4 \theta}+r_{1} \rightarrow x \rightarrow \text { CPA3 }
$$
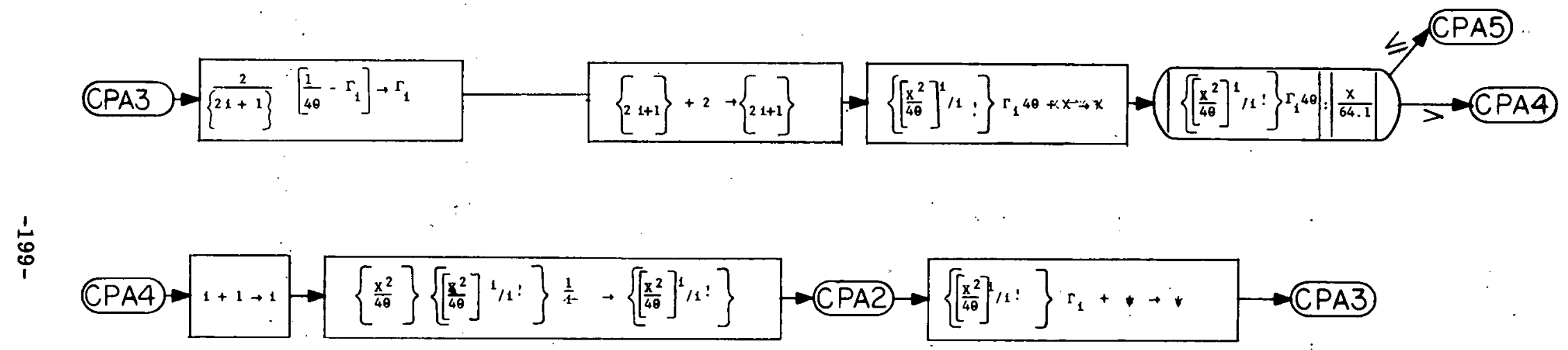

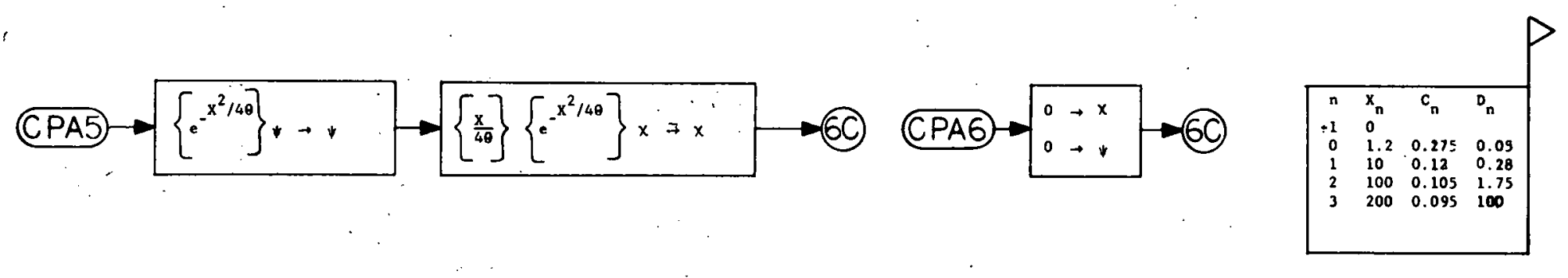


PRE-DIFFUSION .
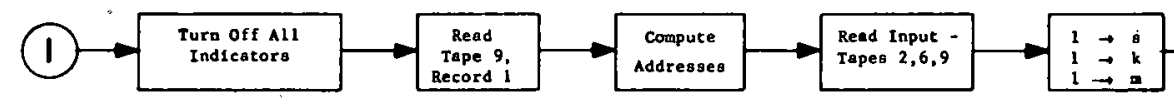

(2) $\rightarrow r_{\mathrm{Hc}_{s}: 1}=(3$
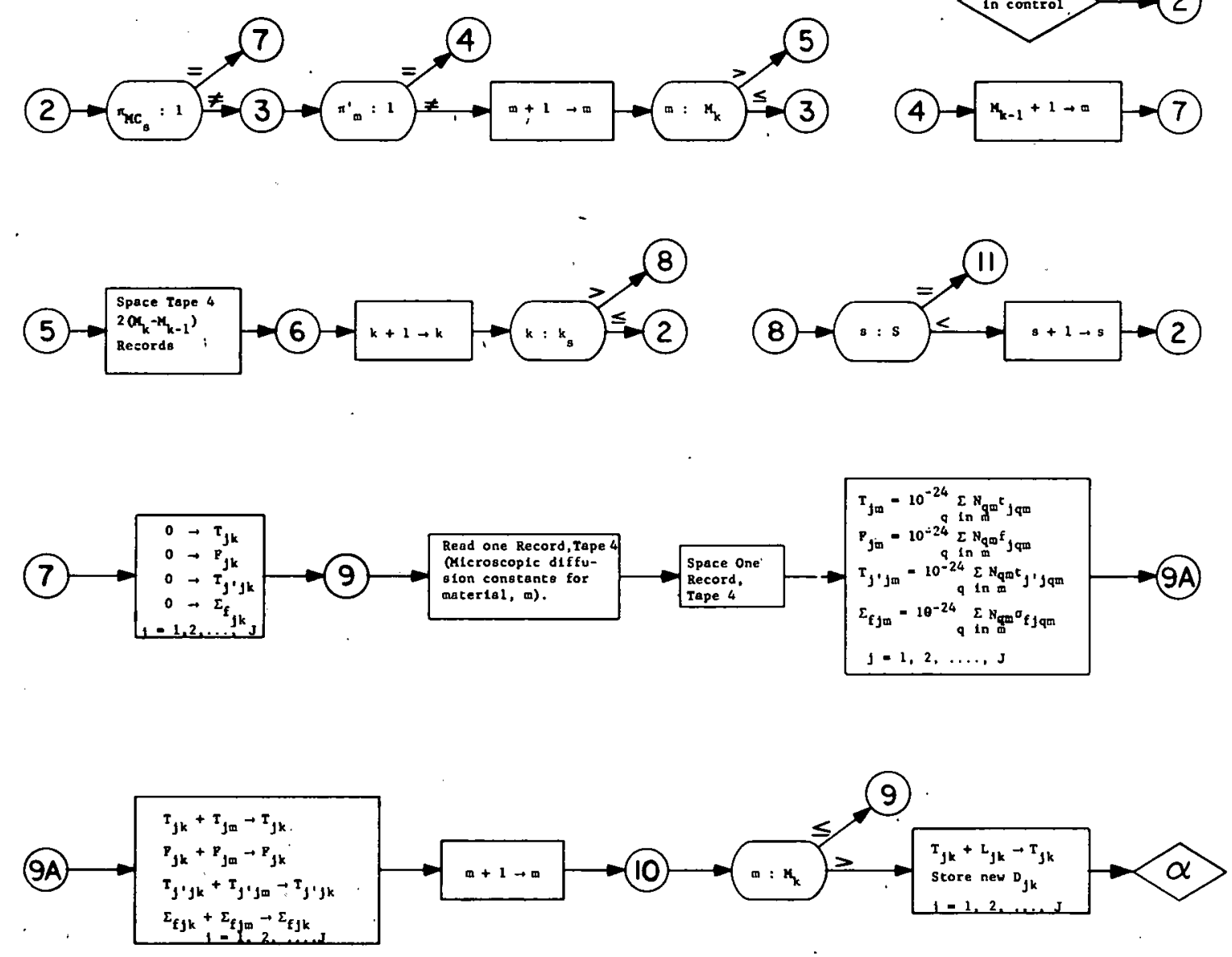

(11) $\rightarrow \begin{gathered}\text { REN } \\ \text { Tape 4 }\end{gathered} \rightarrow \begin{aligned} & \text { TO } \\ & \text { DIFFUSION } \\ & \text { CONTROL } \\ & \text { ROUTINE }\end{aligned} \rightarrow$ (12) 


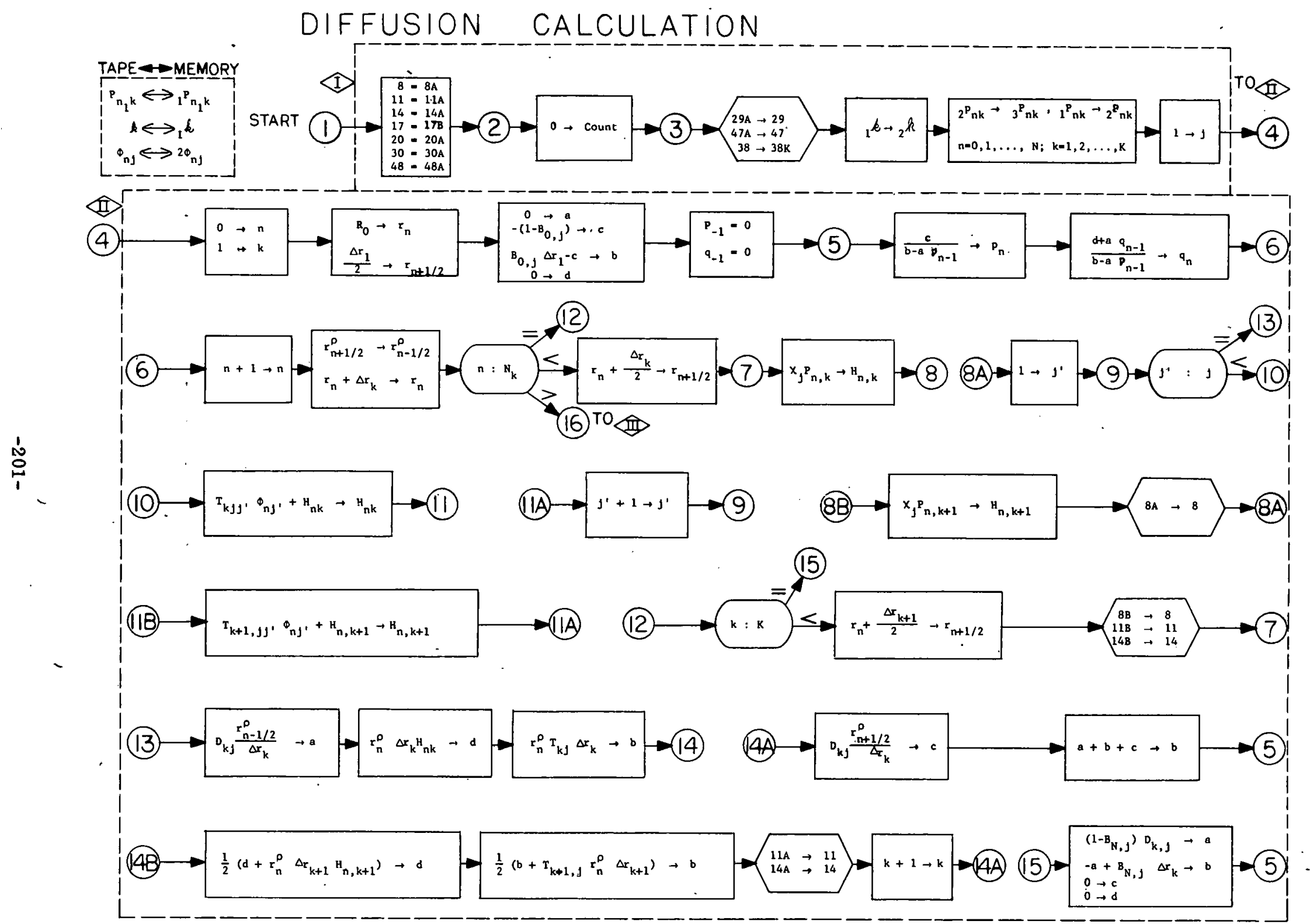




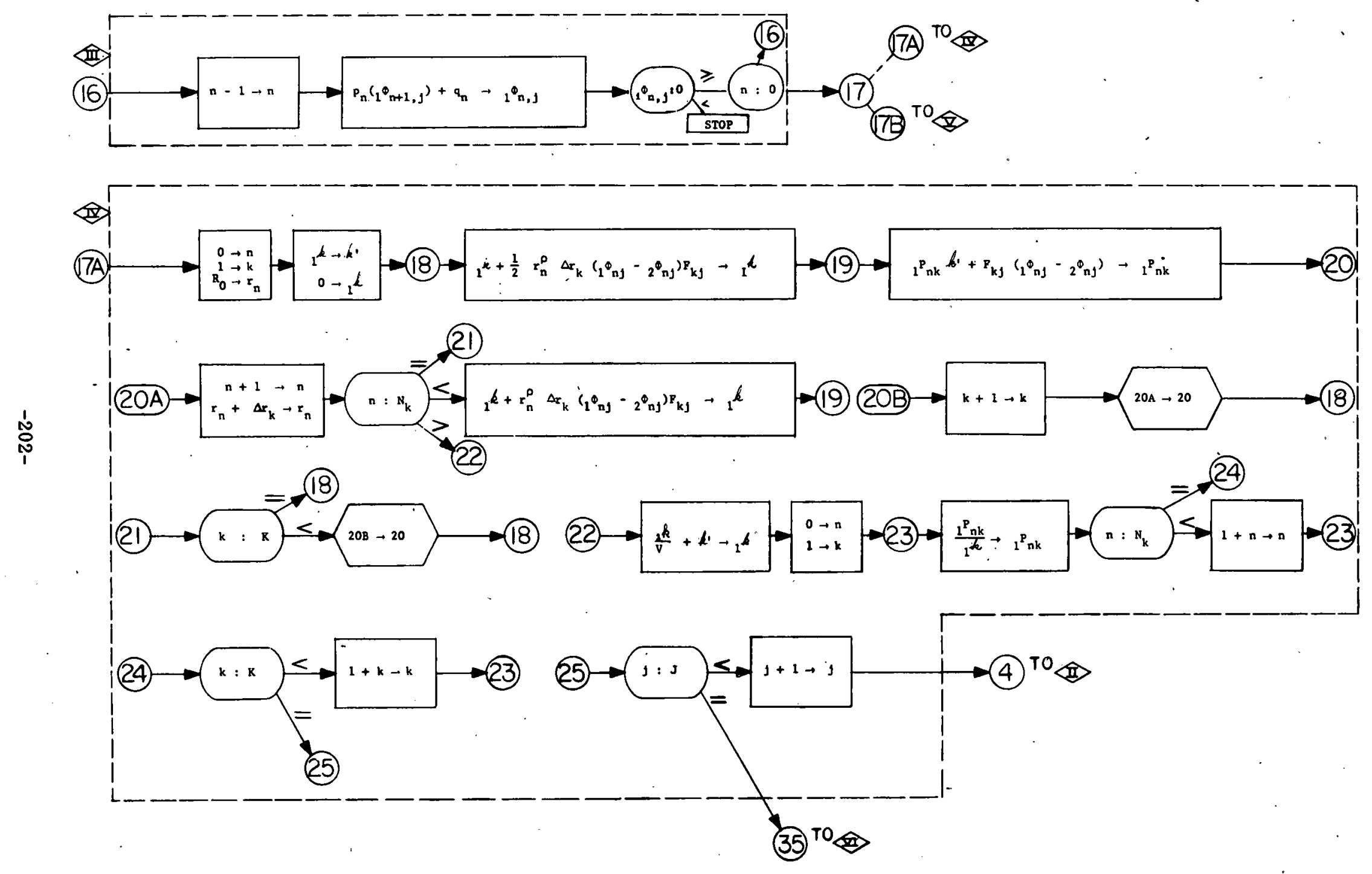




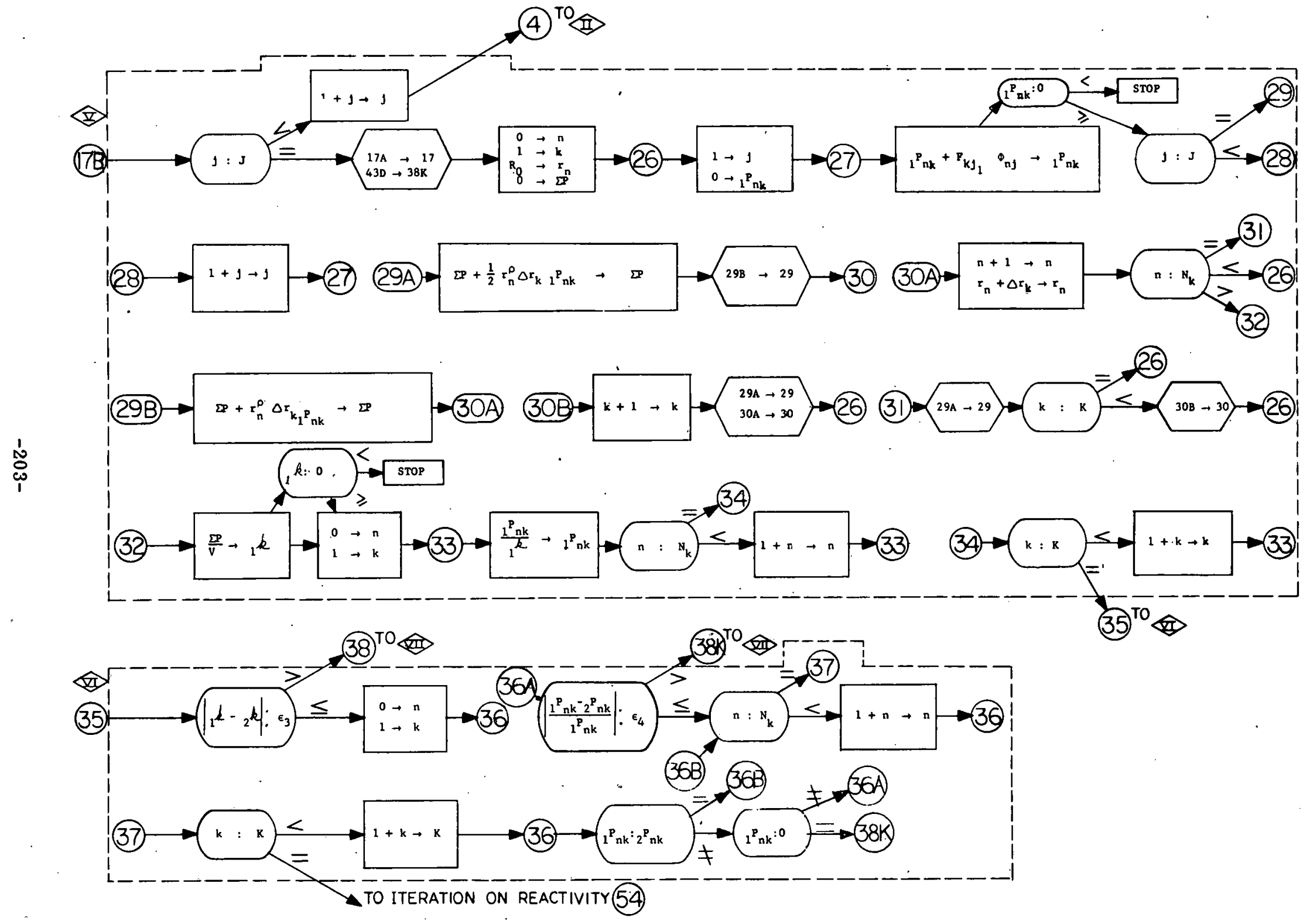




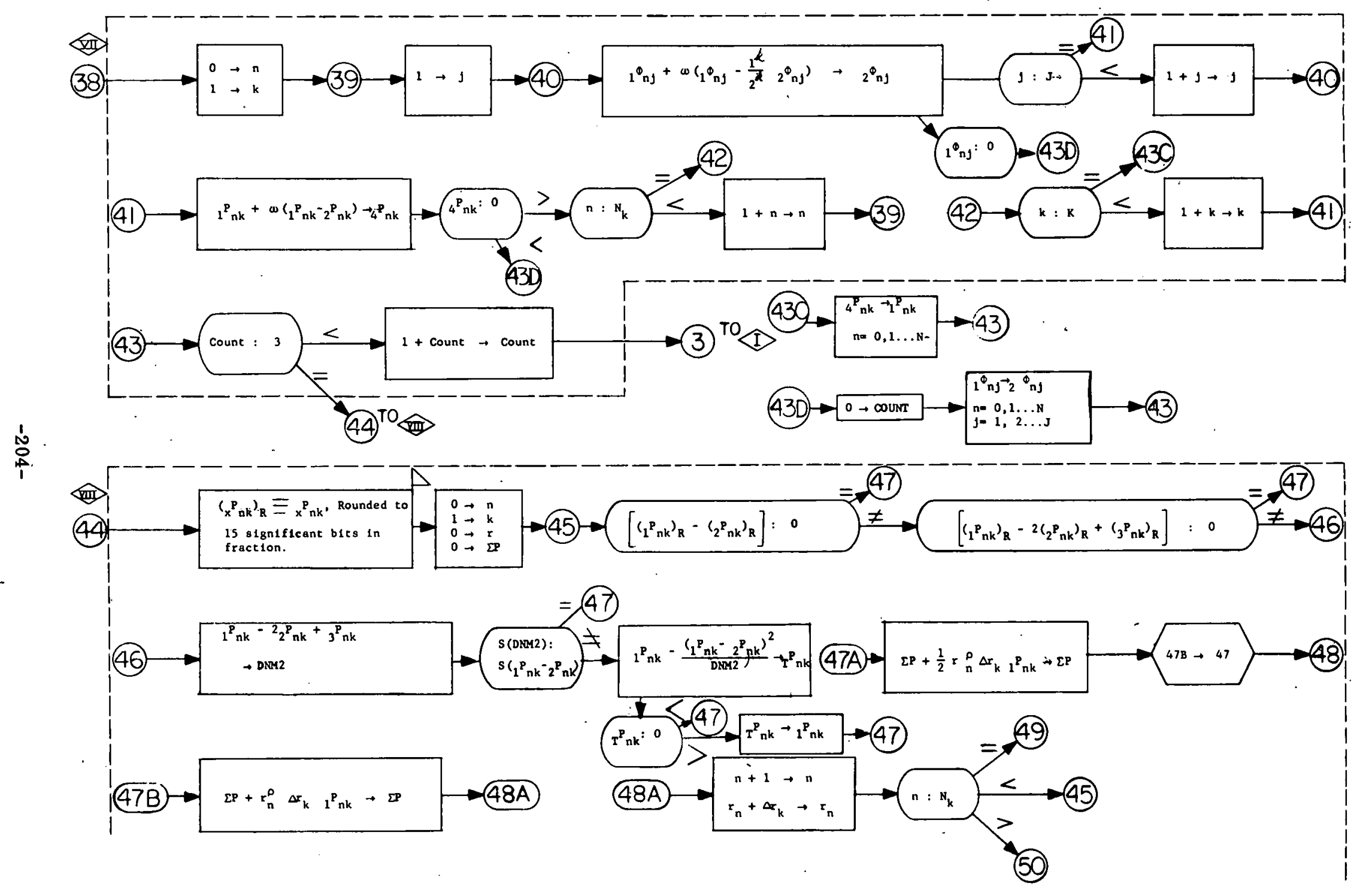




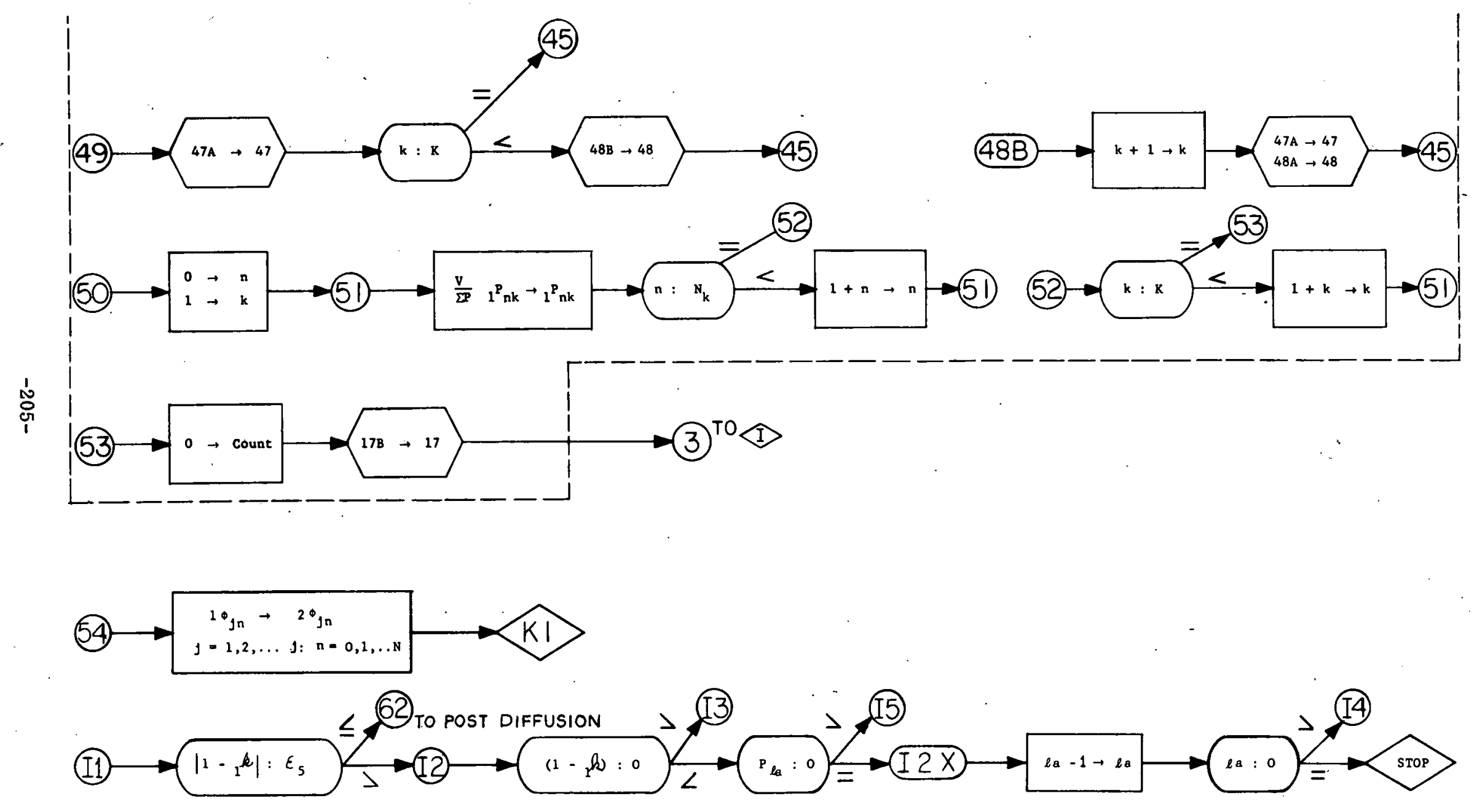




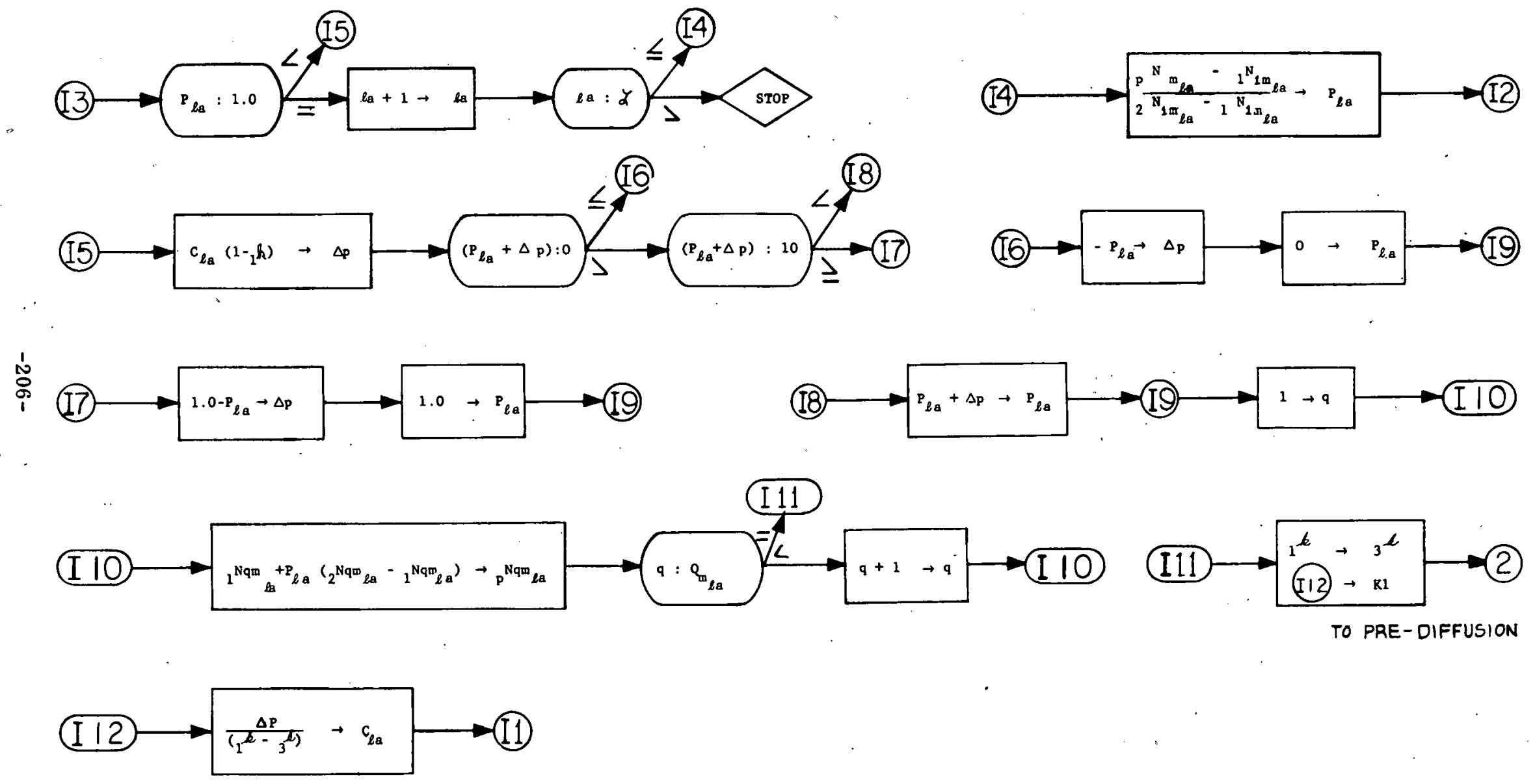




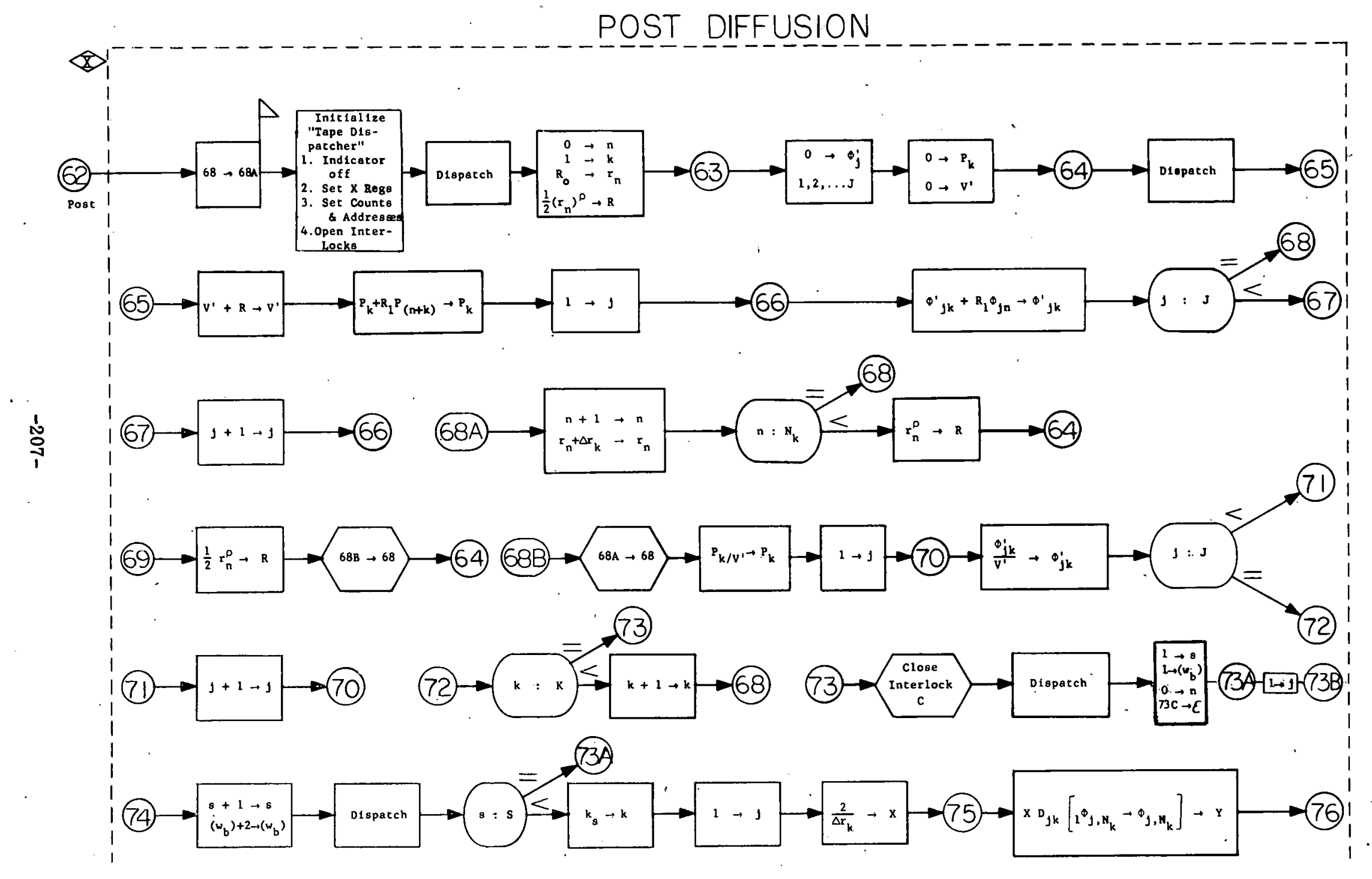




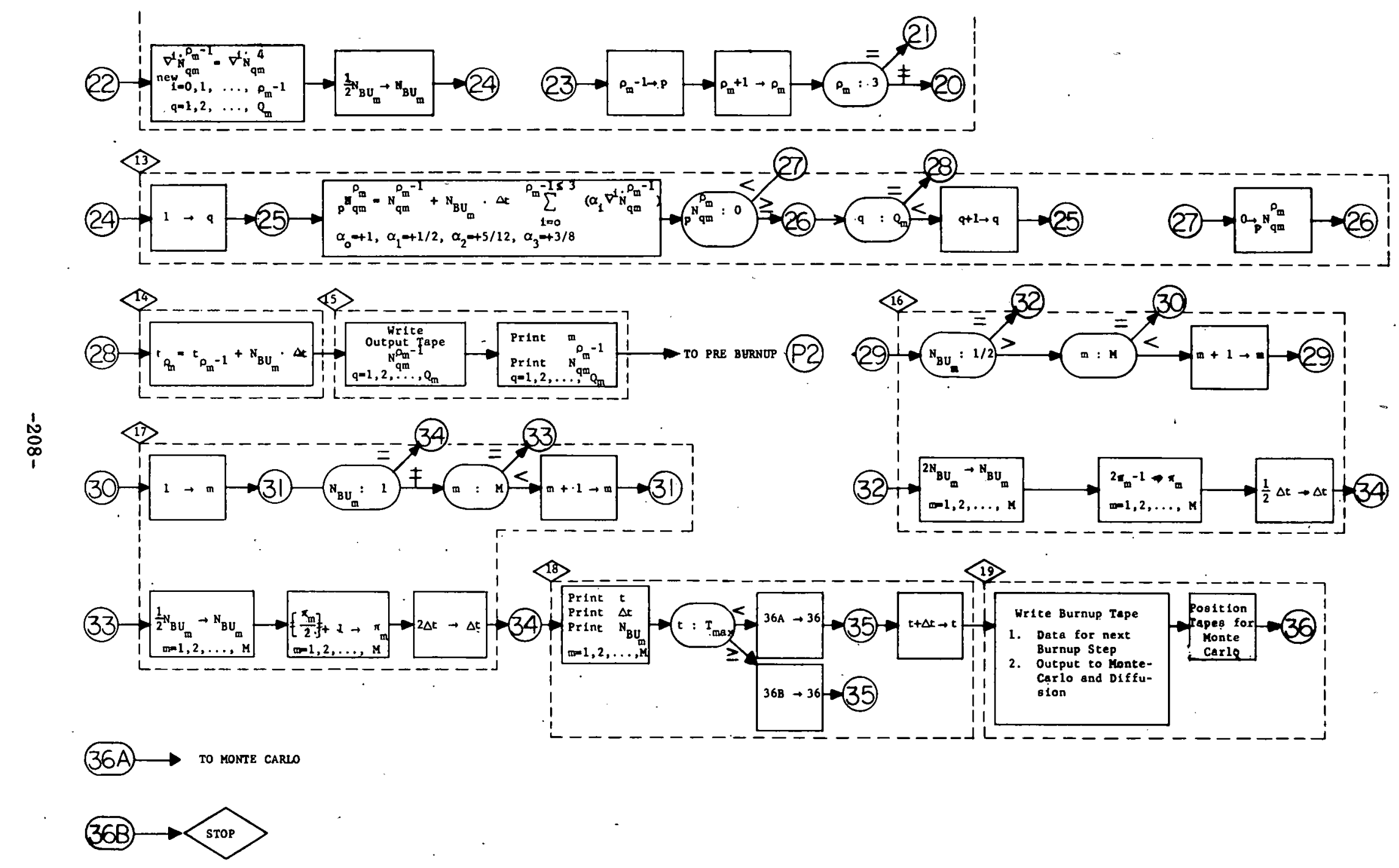


(4)

P

(4)-

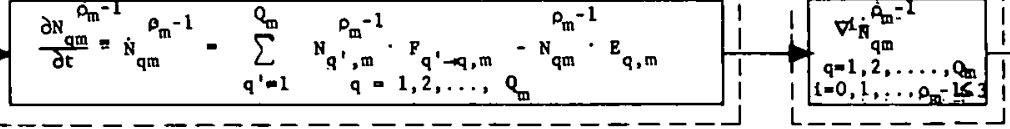

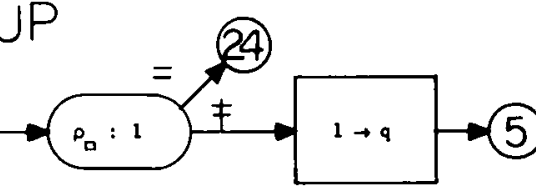

(3) $\rightarrow+2+\cdots+$ (2)

(5)

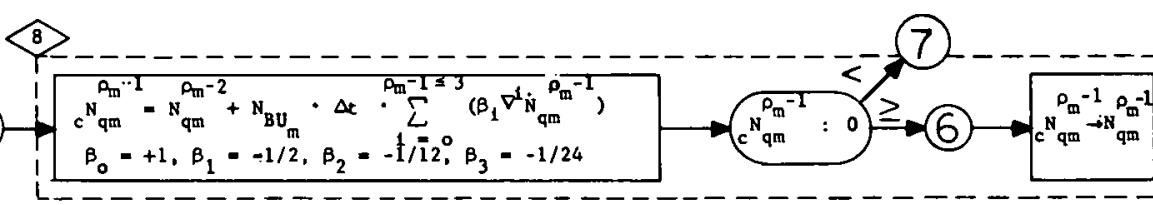

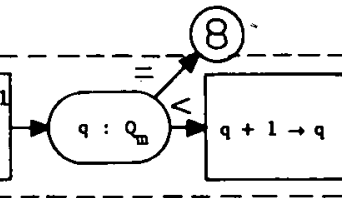

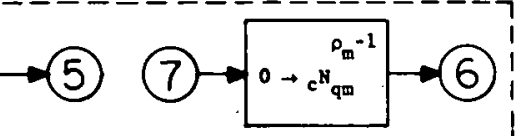

(8) $\rightarrow+9$

(II)

$(12$

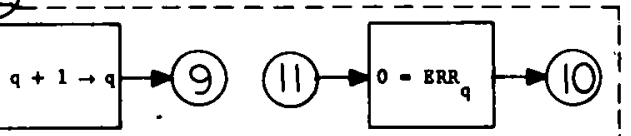

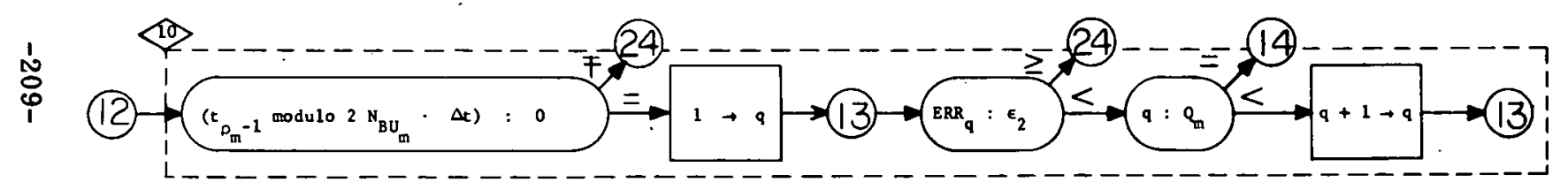

(14)

(17) $\rightarrow$

(18)

(18) $\rightarrow-2 \rightarrow \infty$

(19)

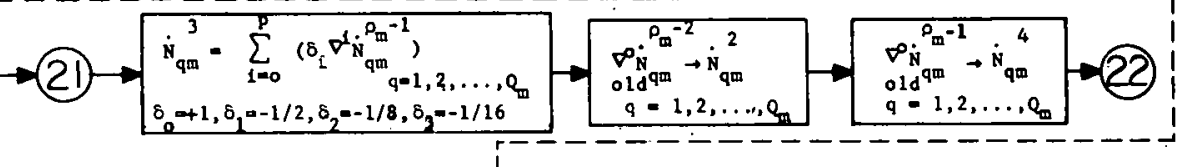




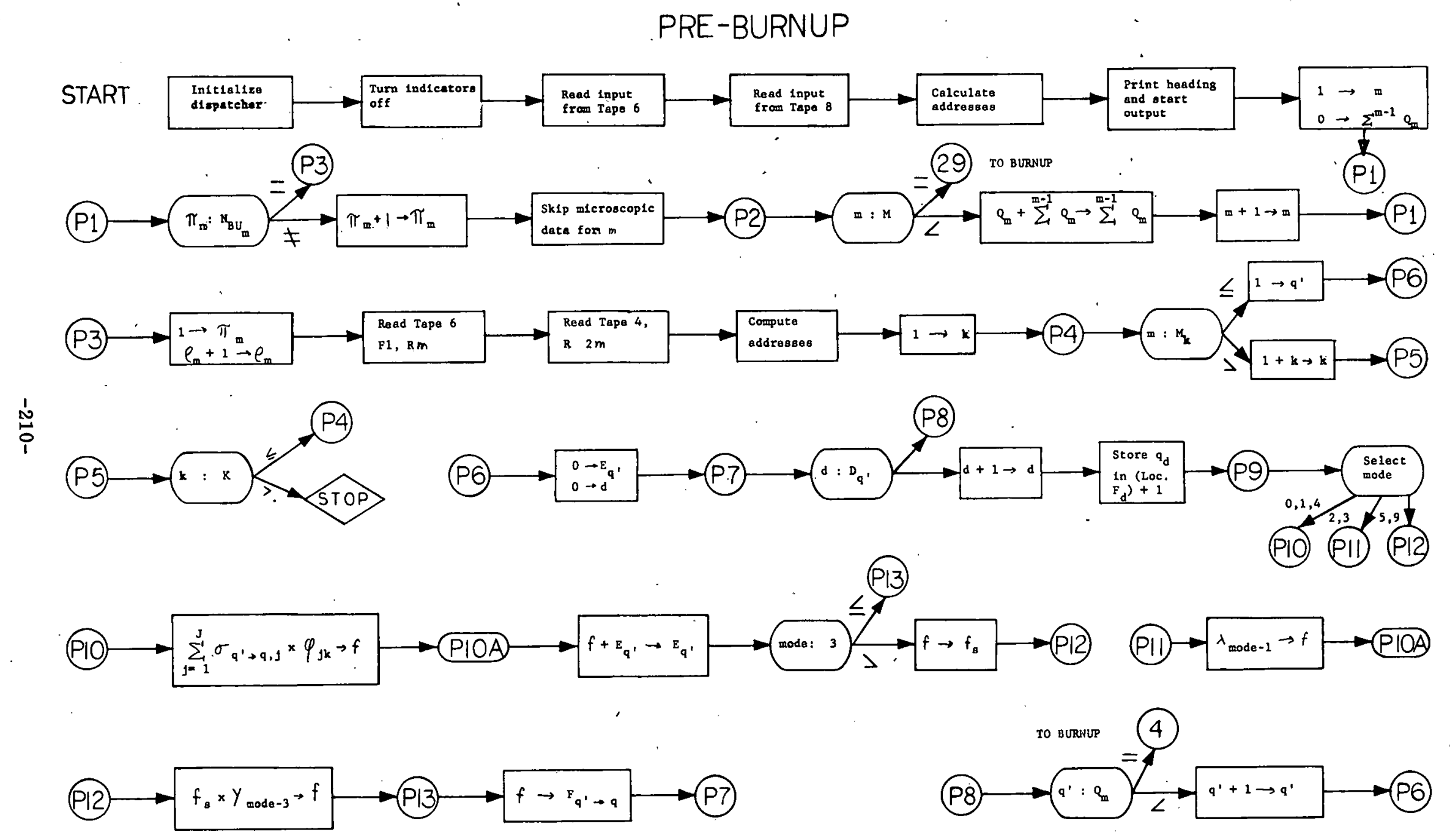




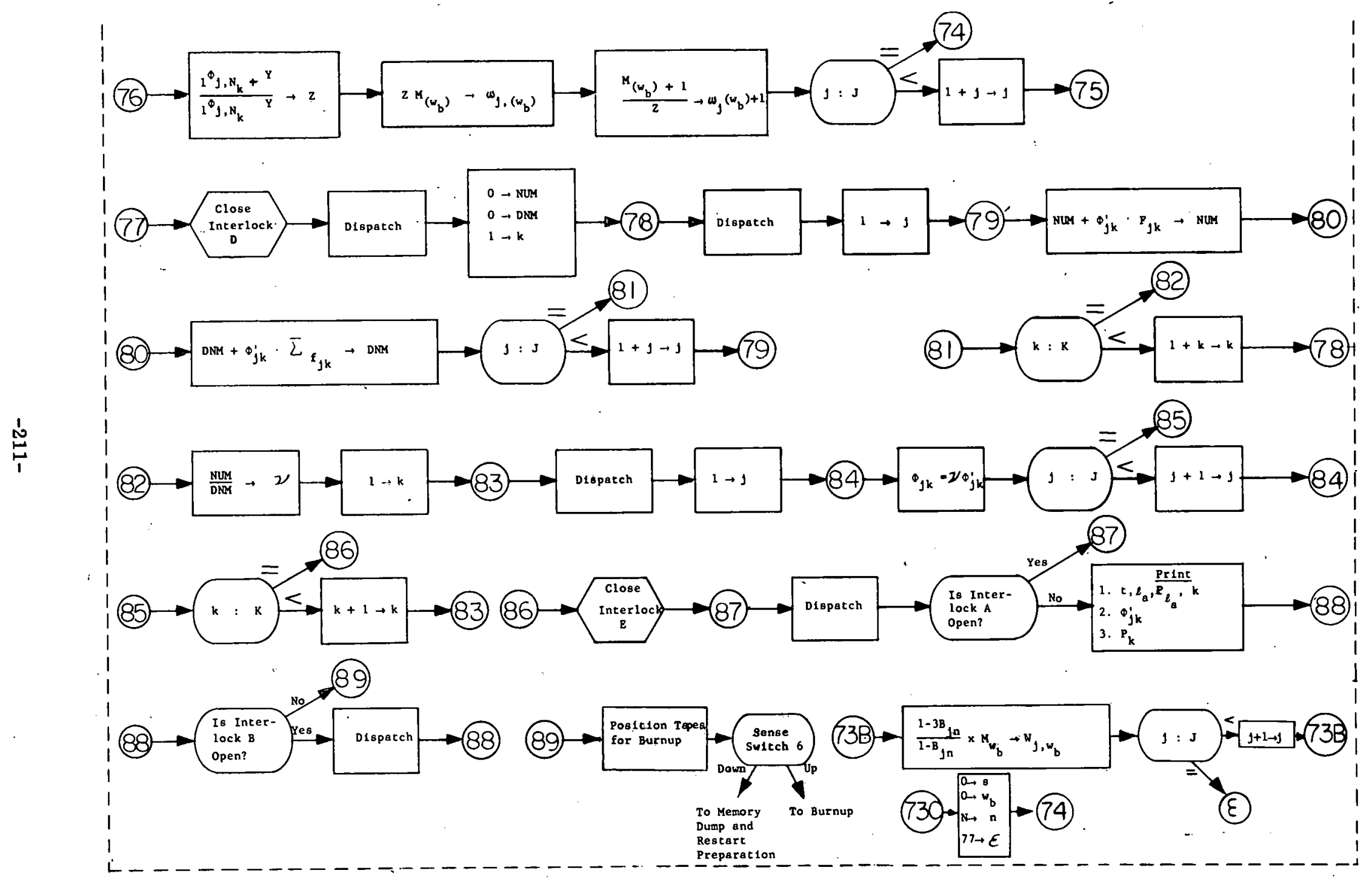




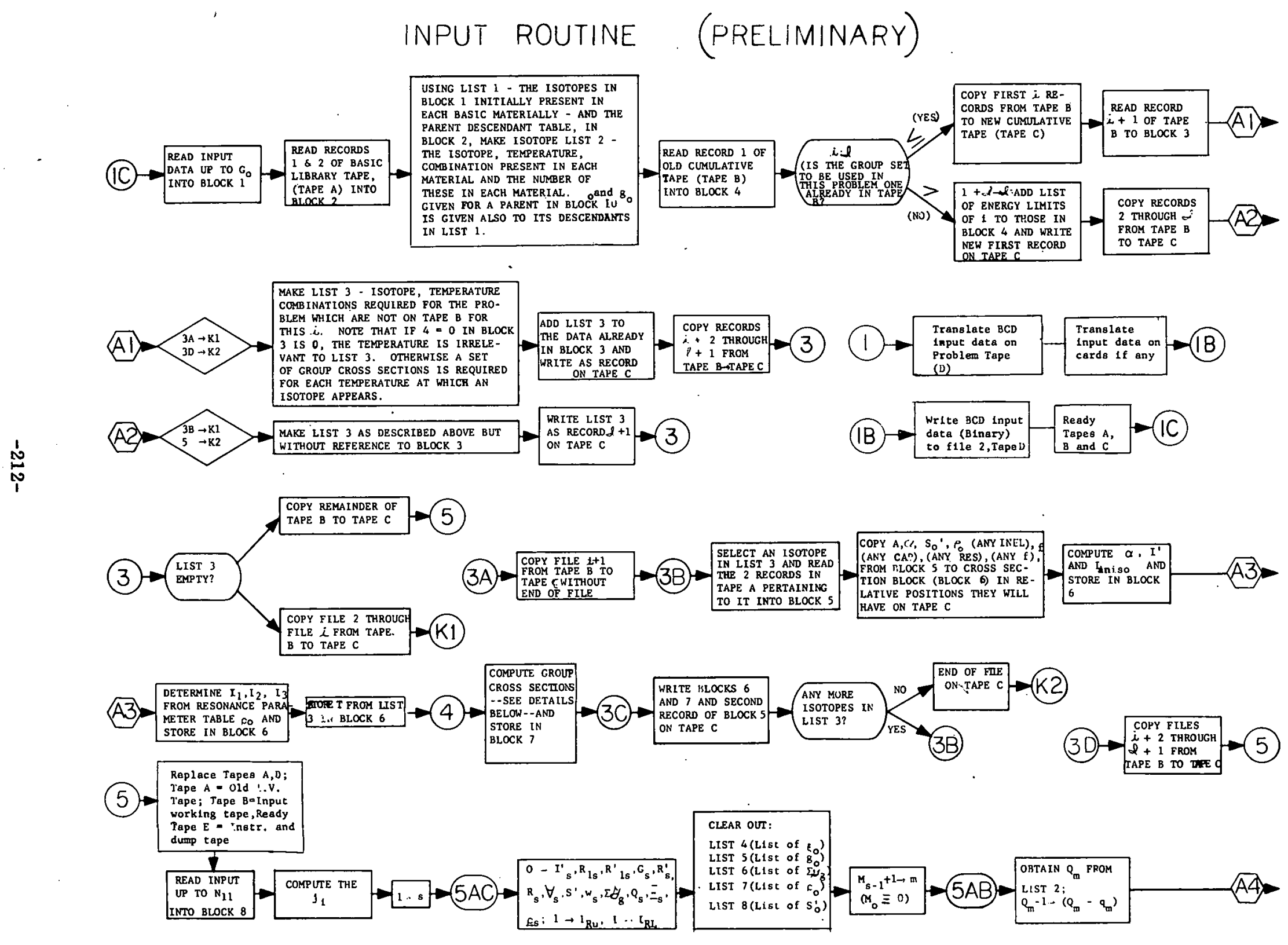




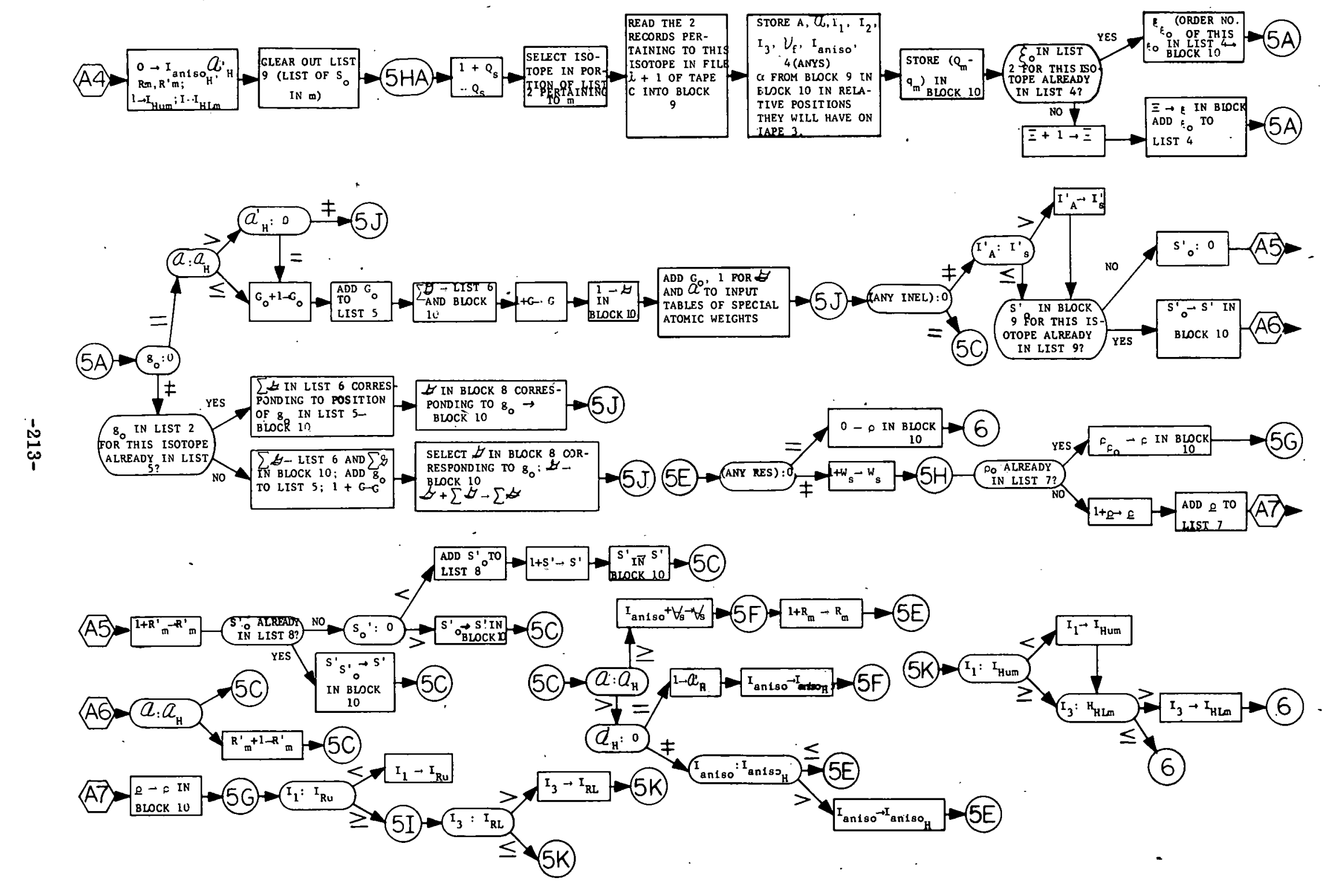




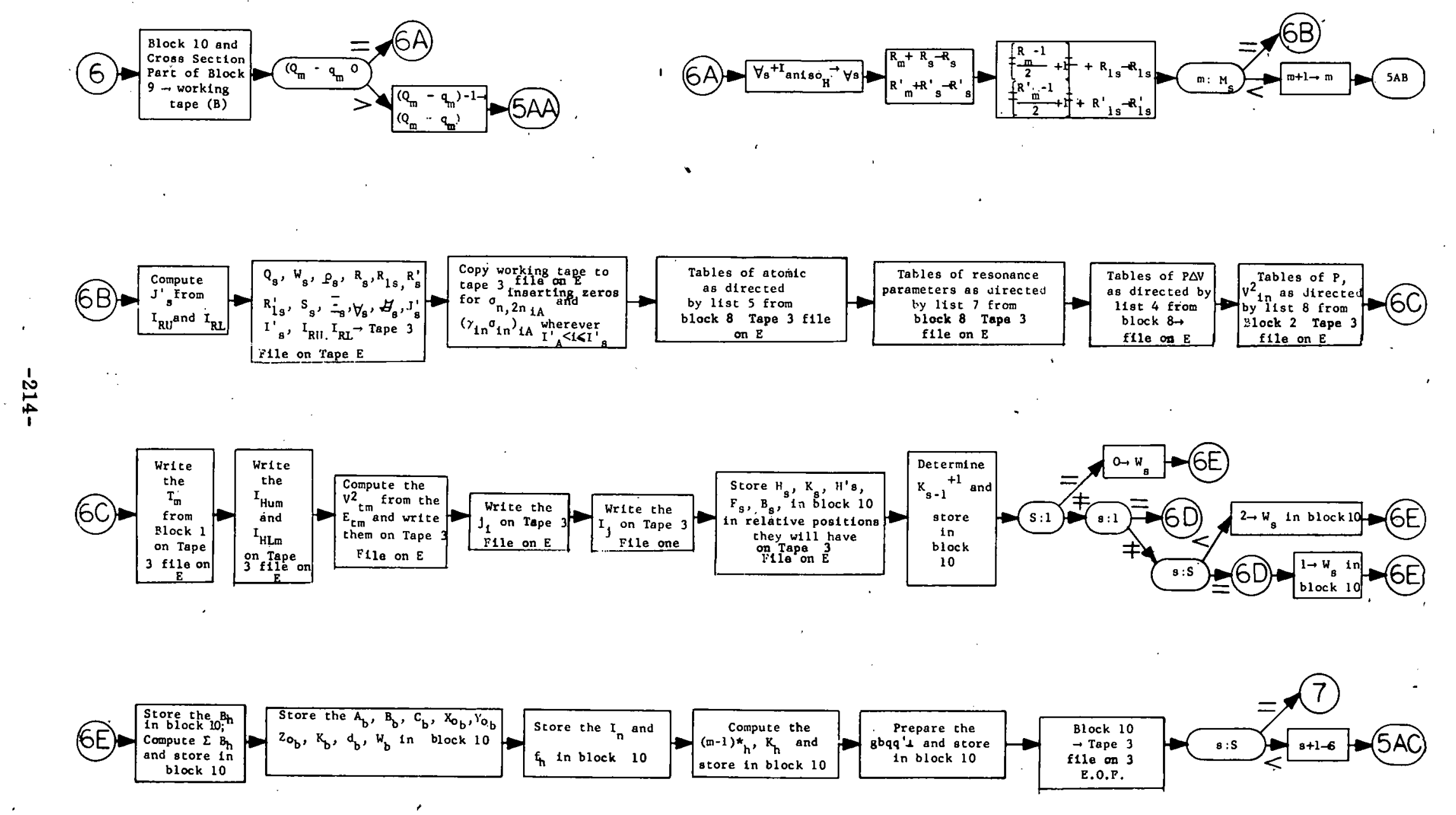




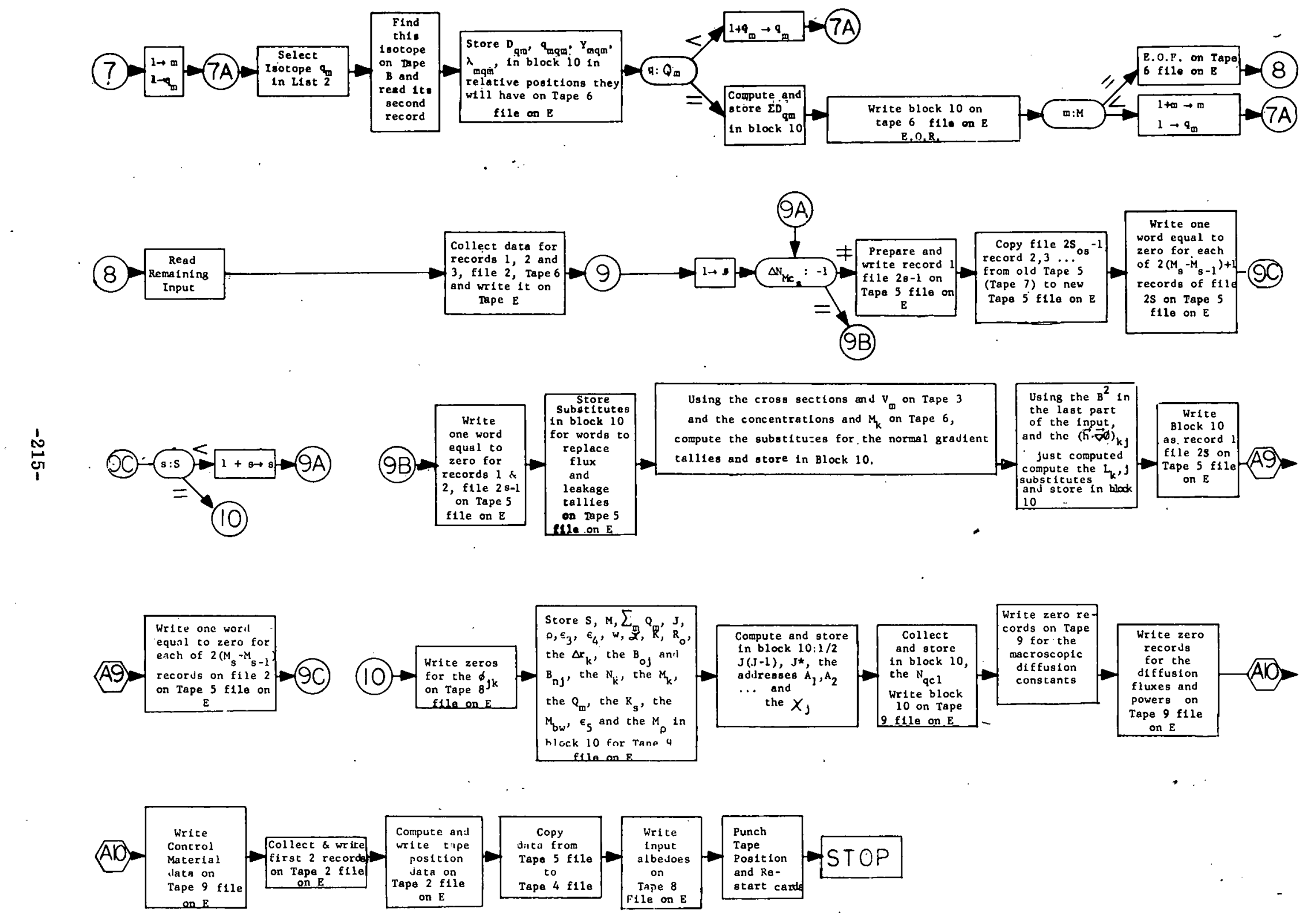


(4)

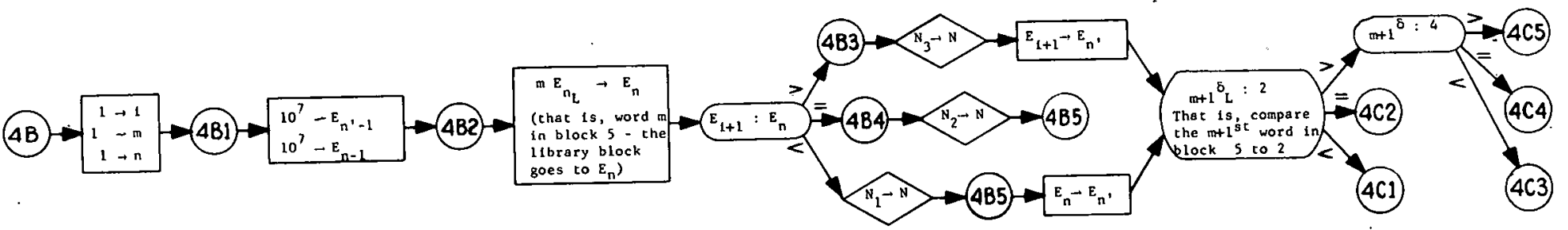
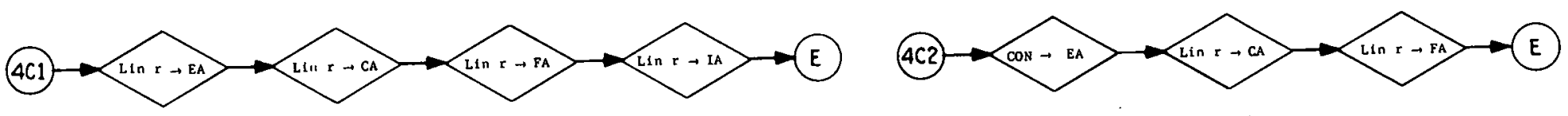

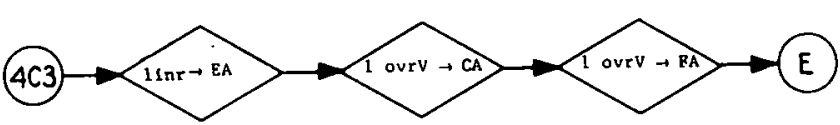

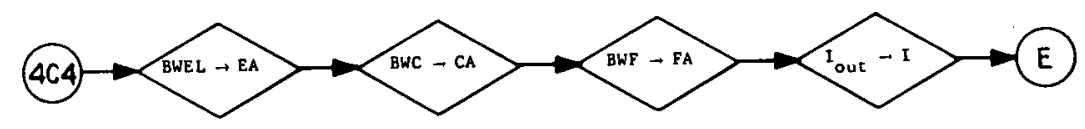

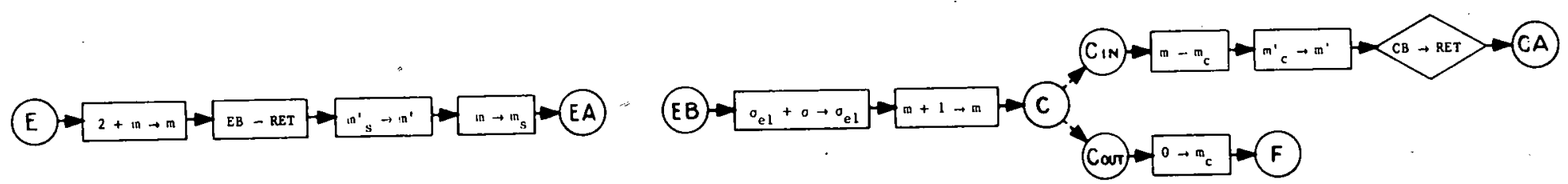

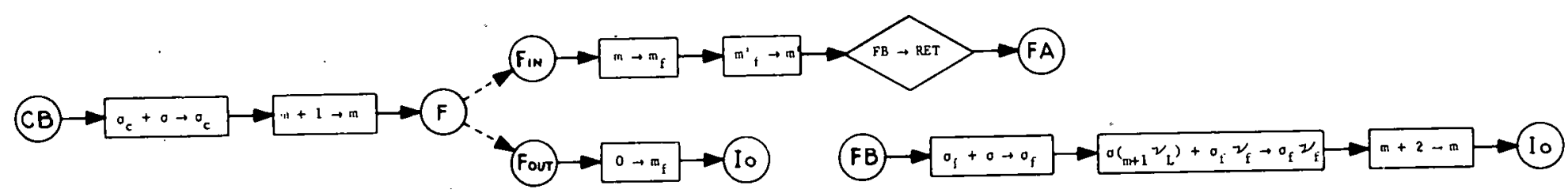




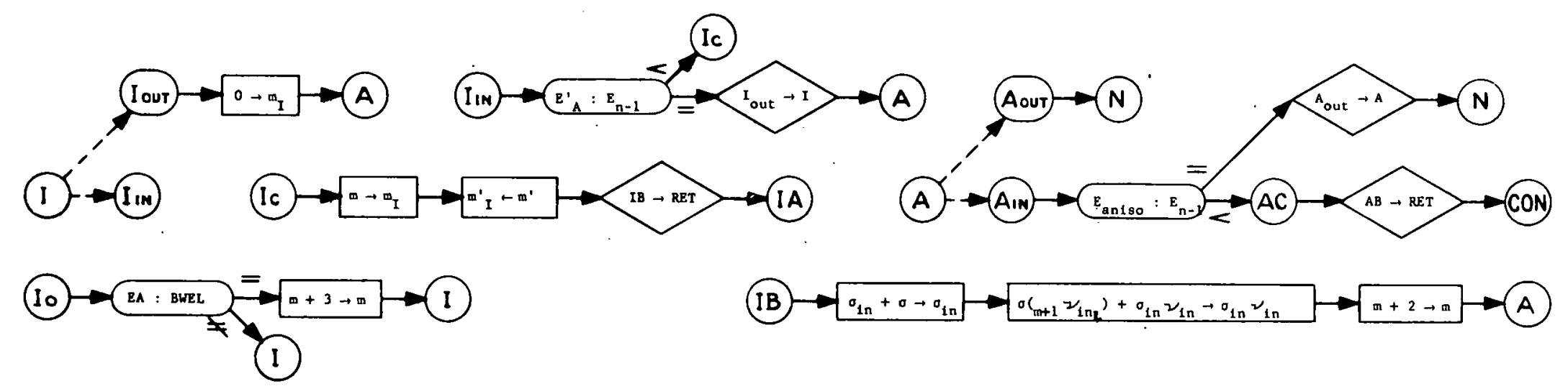

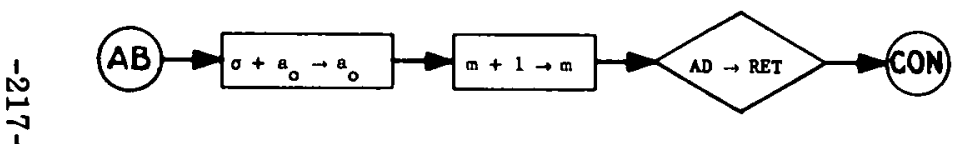

(AD) $\rightarrow O+a_{1}+a_{1} \rightarrow O M+1 \rightarrow m \rightarrow C O B C$

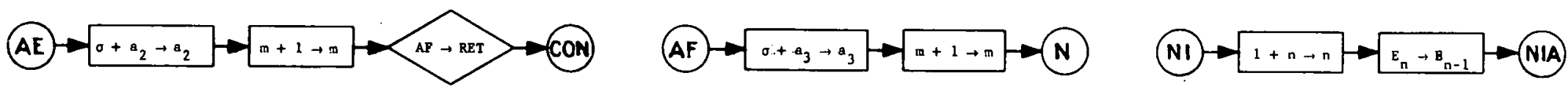

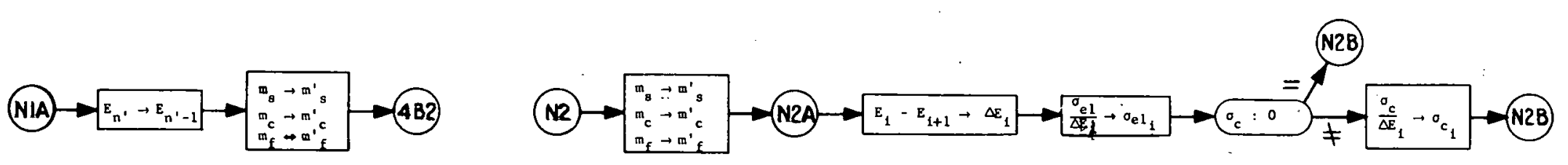

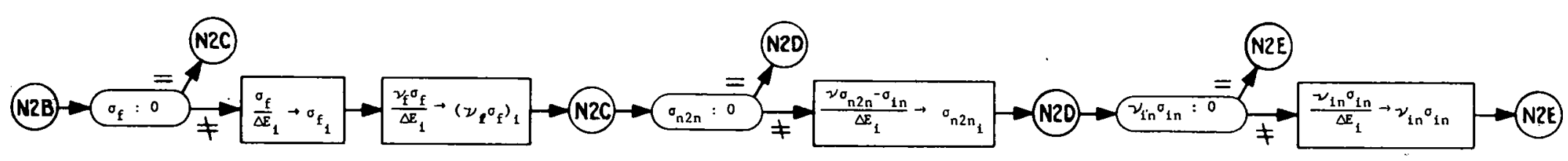




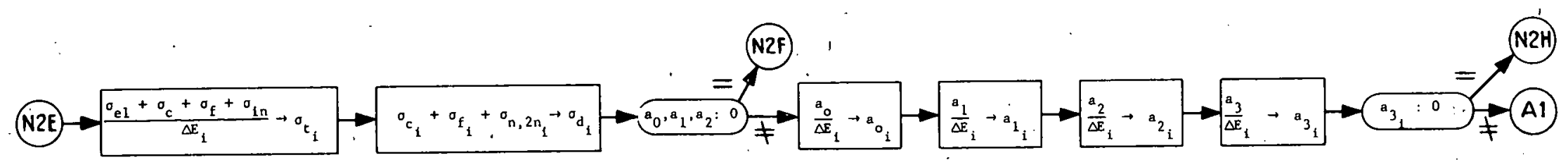

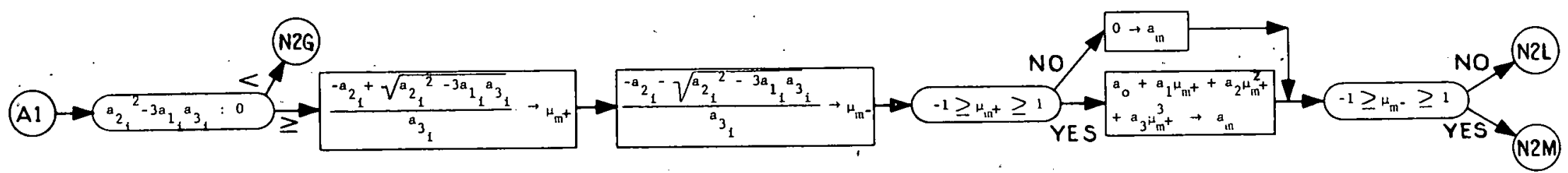

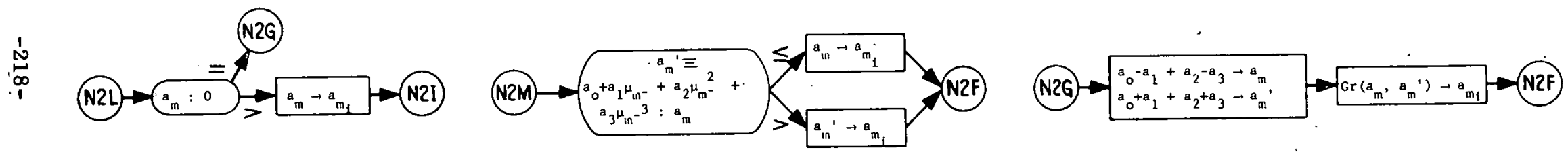

(N2H)

(N3) $\rightarrow m_{s}-3 \rightarrow m \rightarrow N 2$

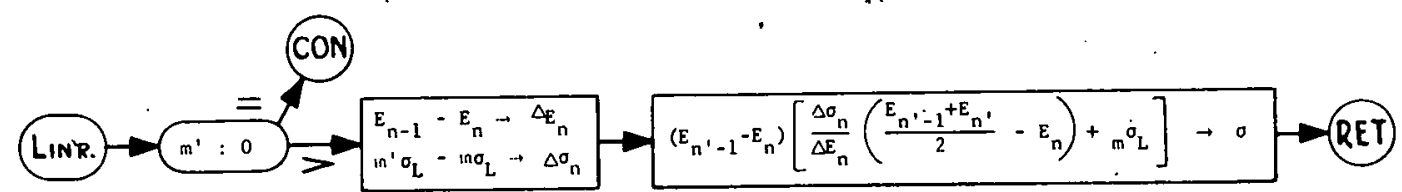




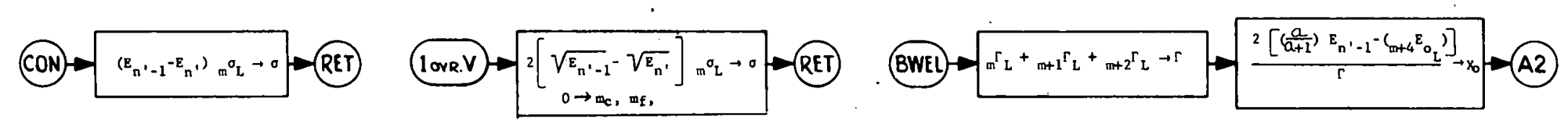

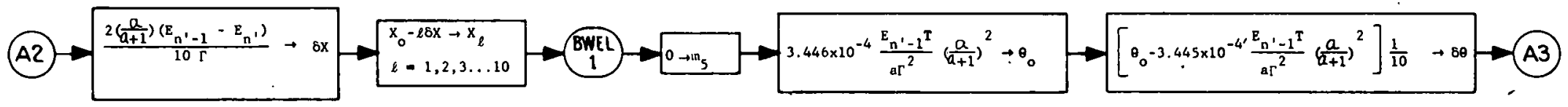

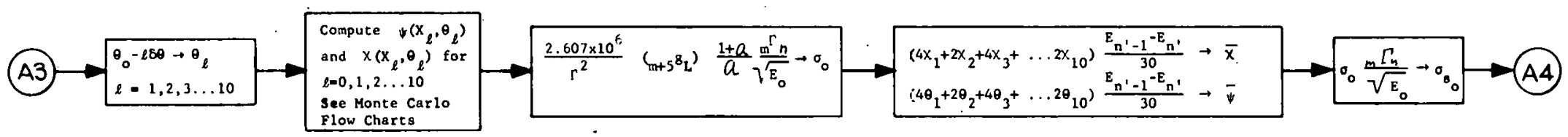
$\underset{\substack{\dot{\omega} \\ \varphi}}{i}$

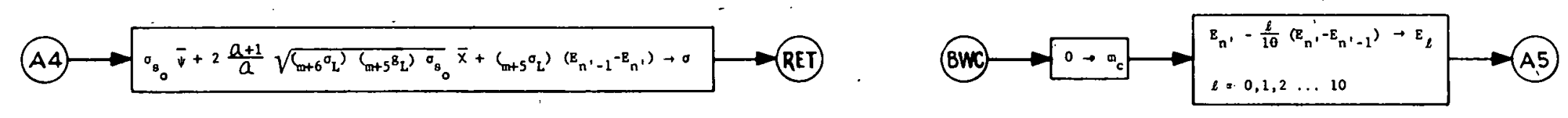

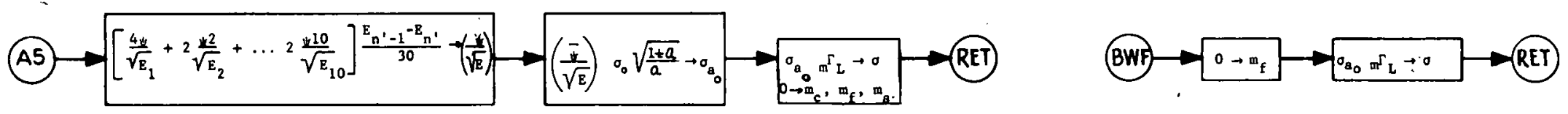

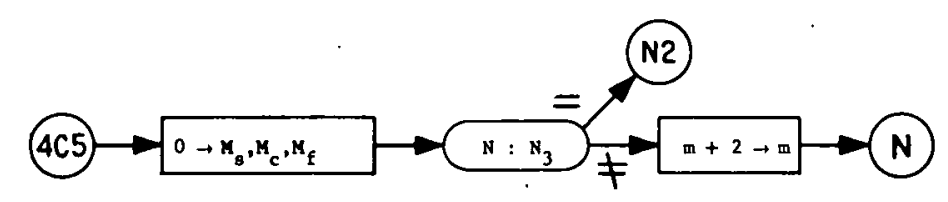


PRE-PRE MONTE CARLO
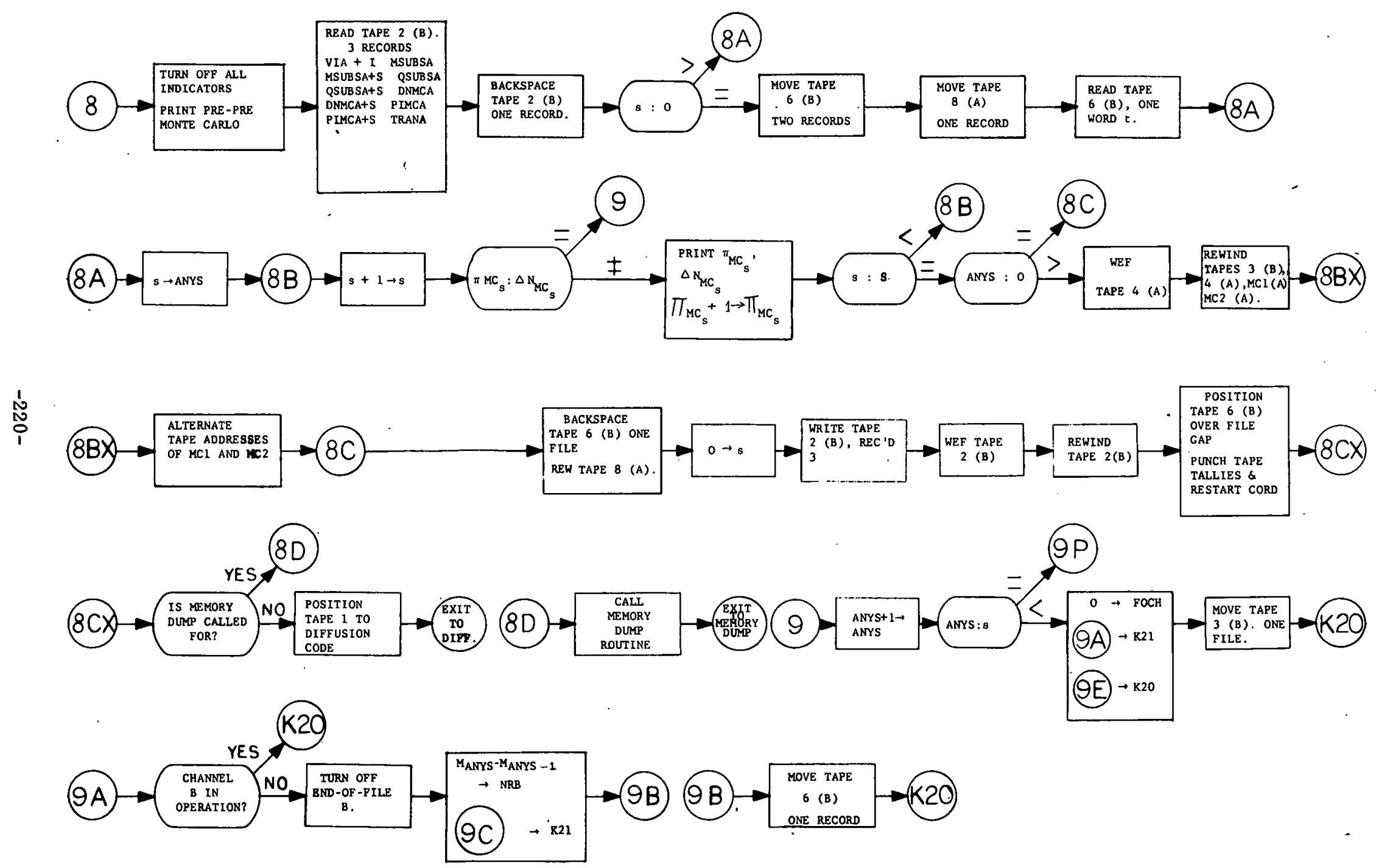


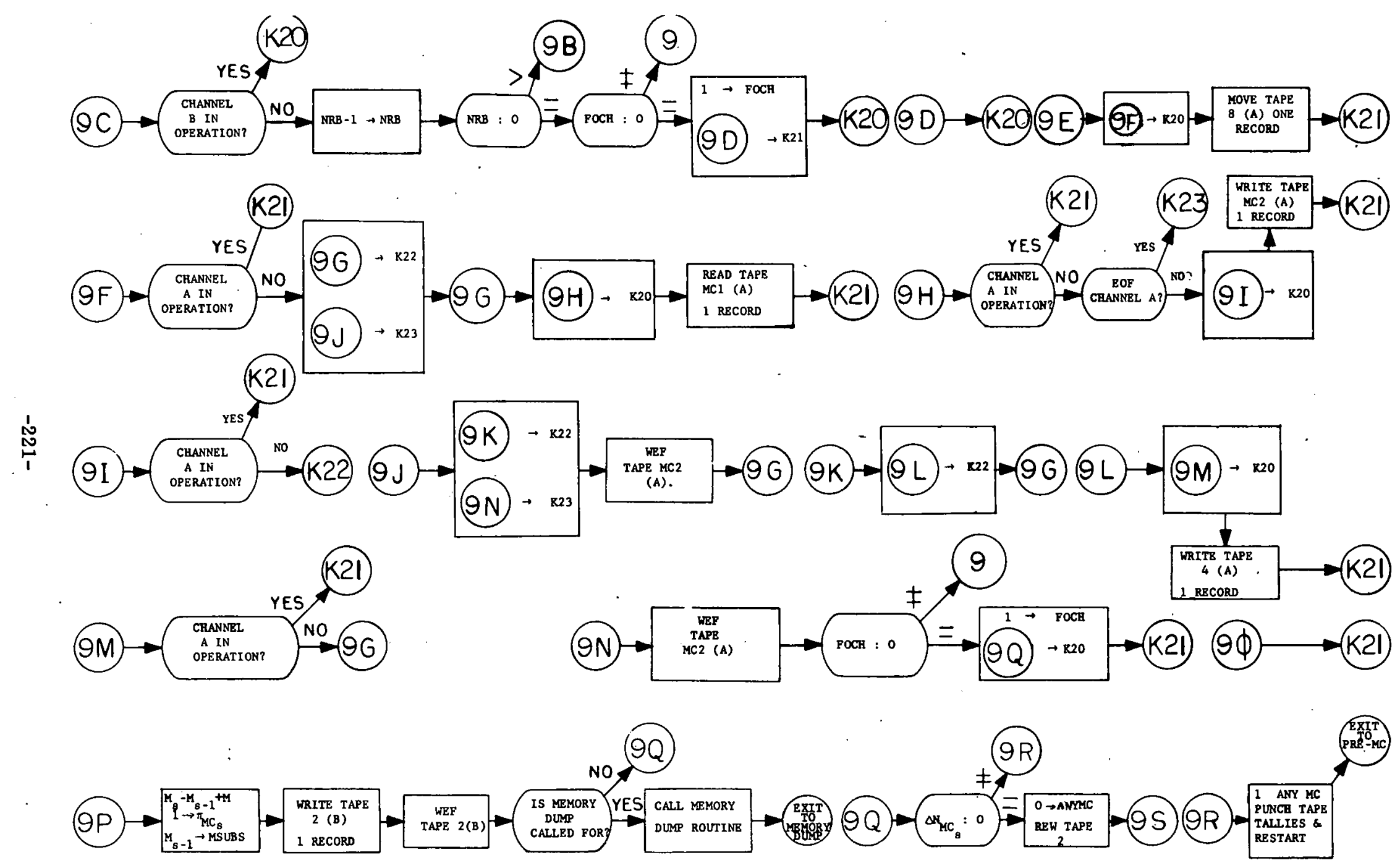



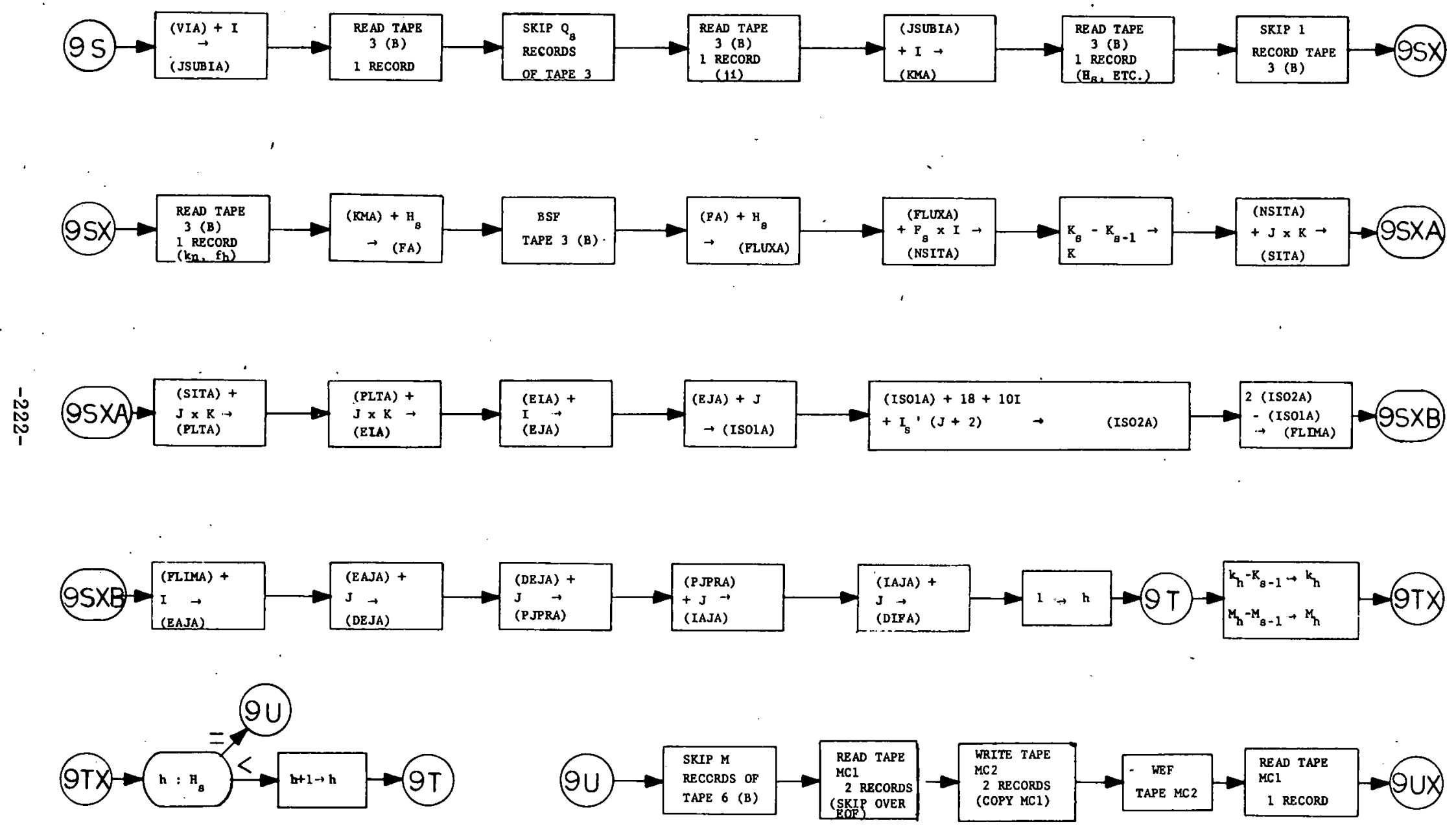


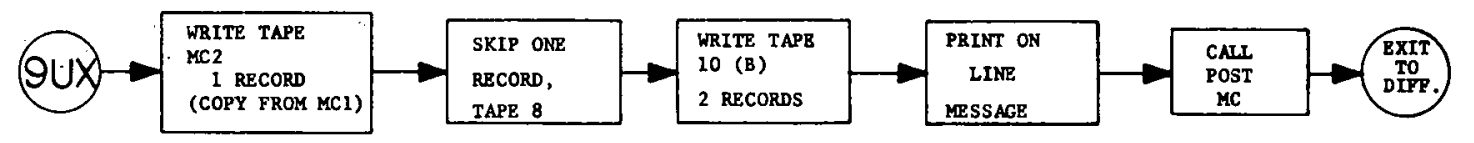

PRE-MONTE CARLO

点
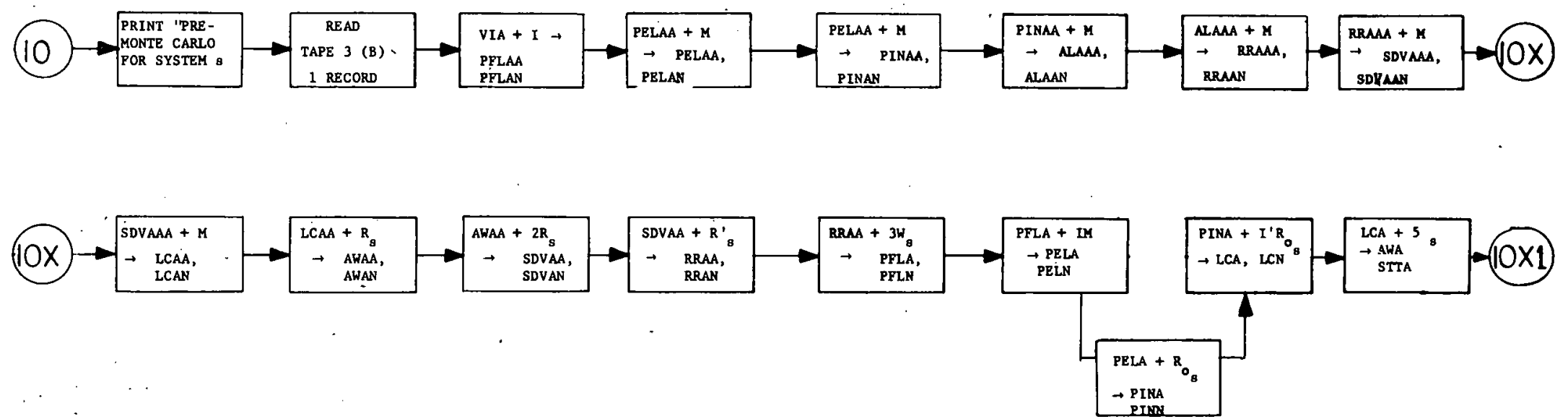

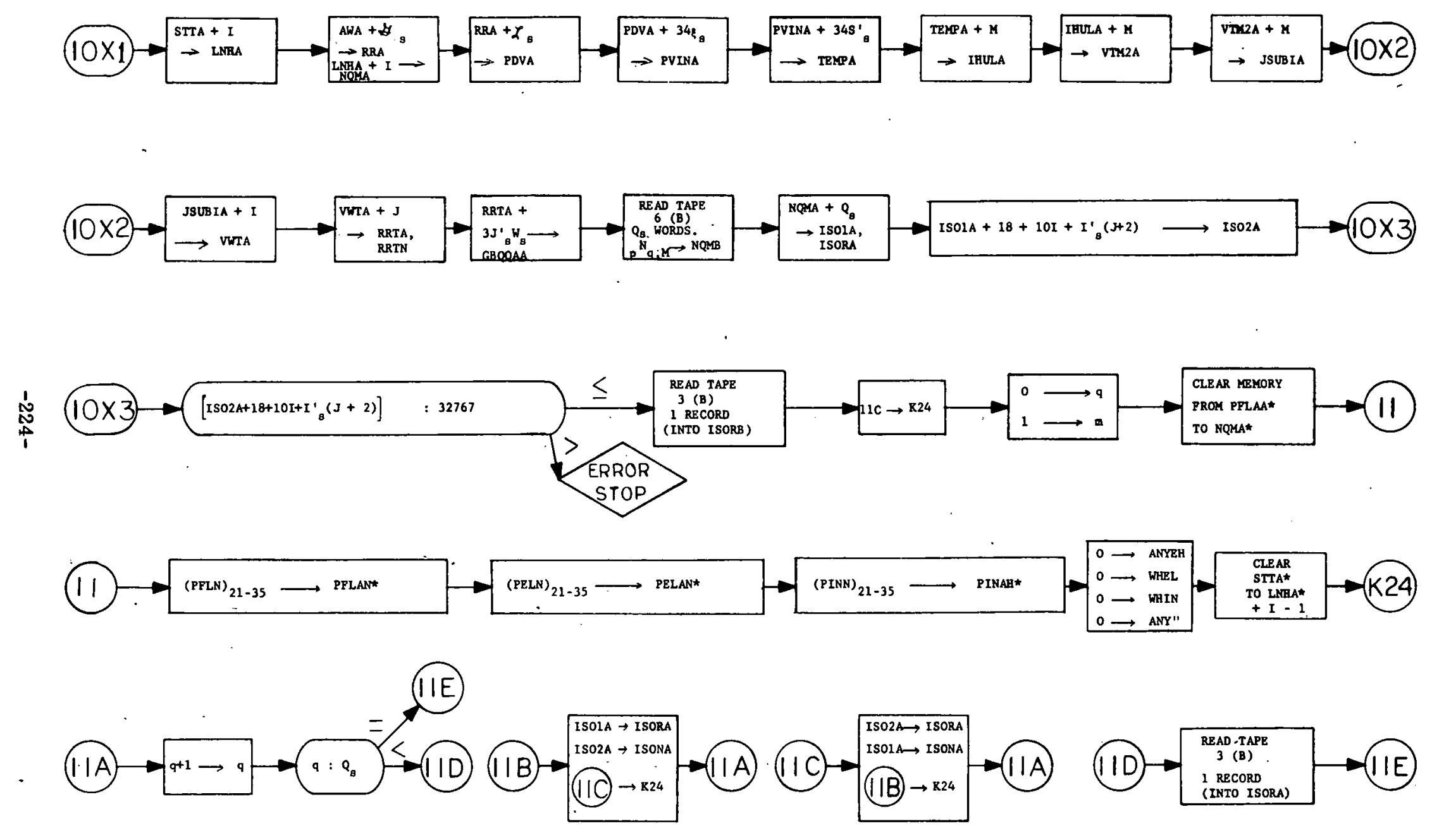

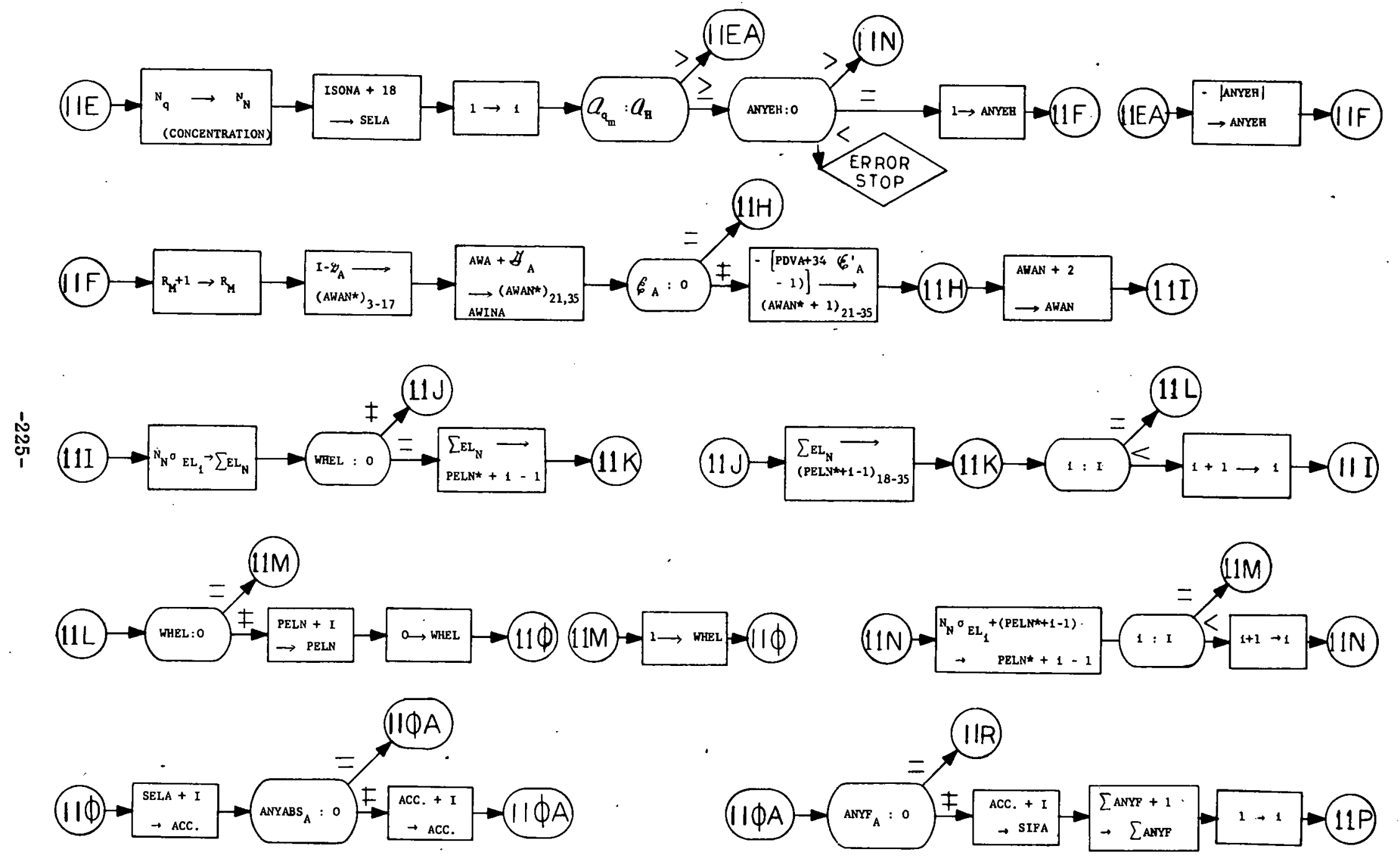


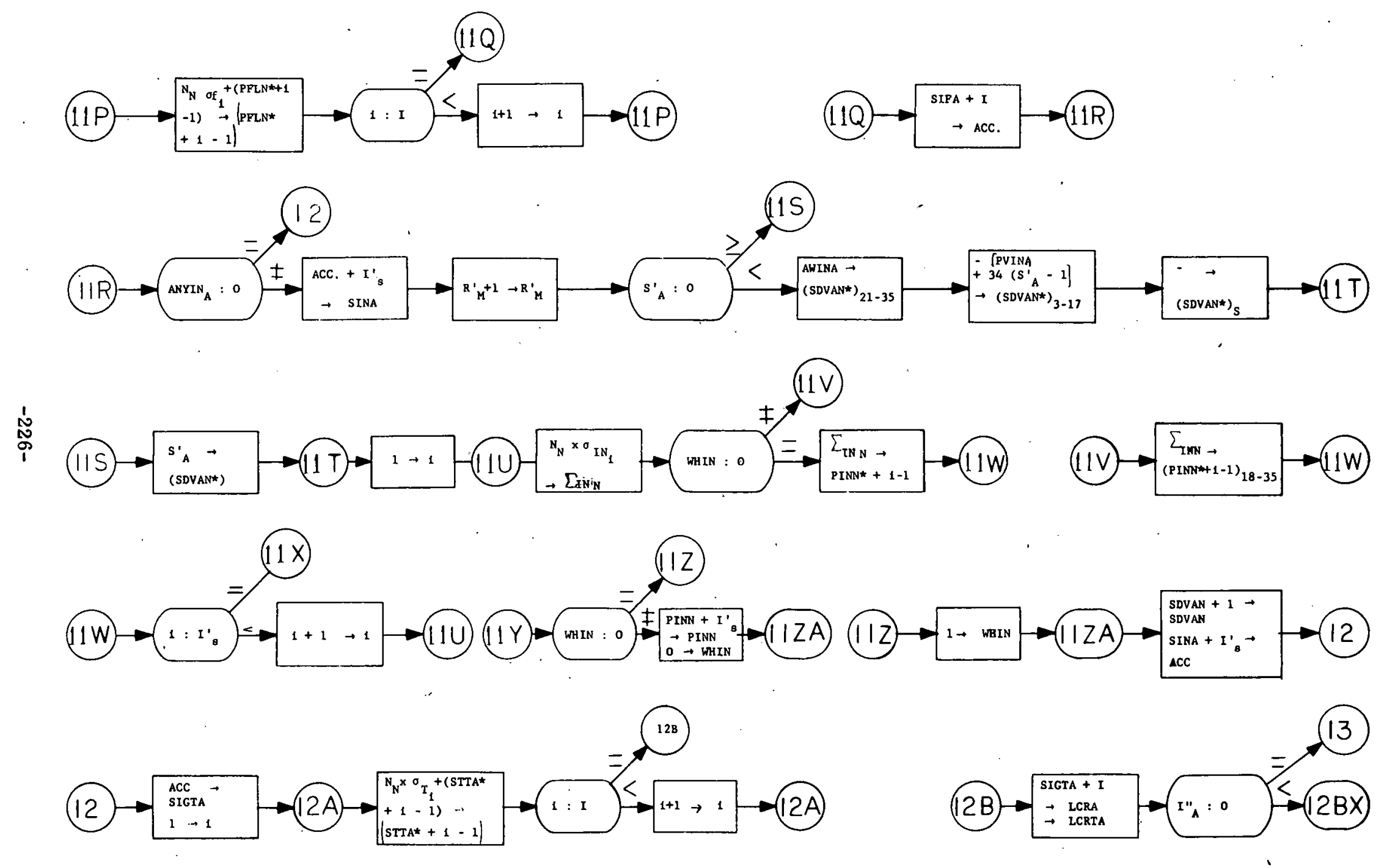




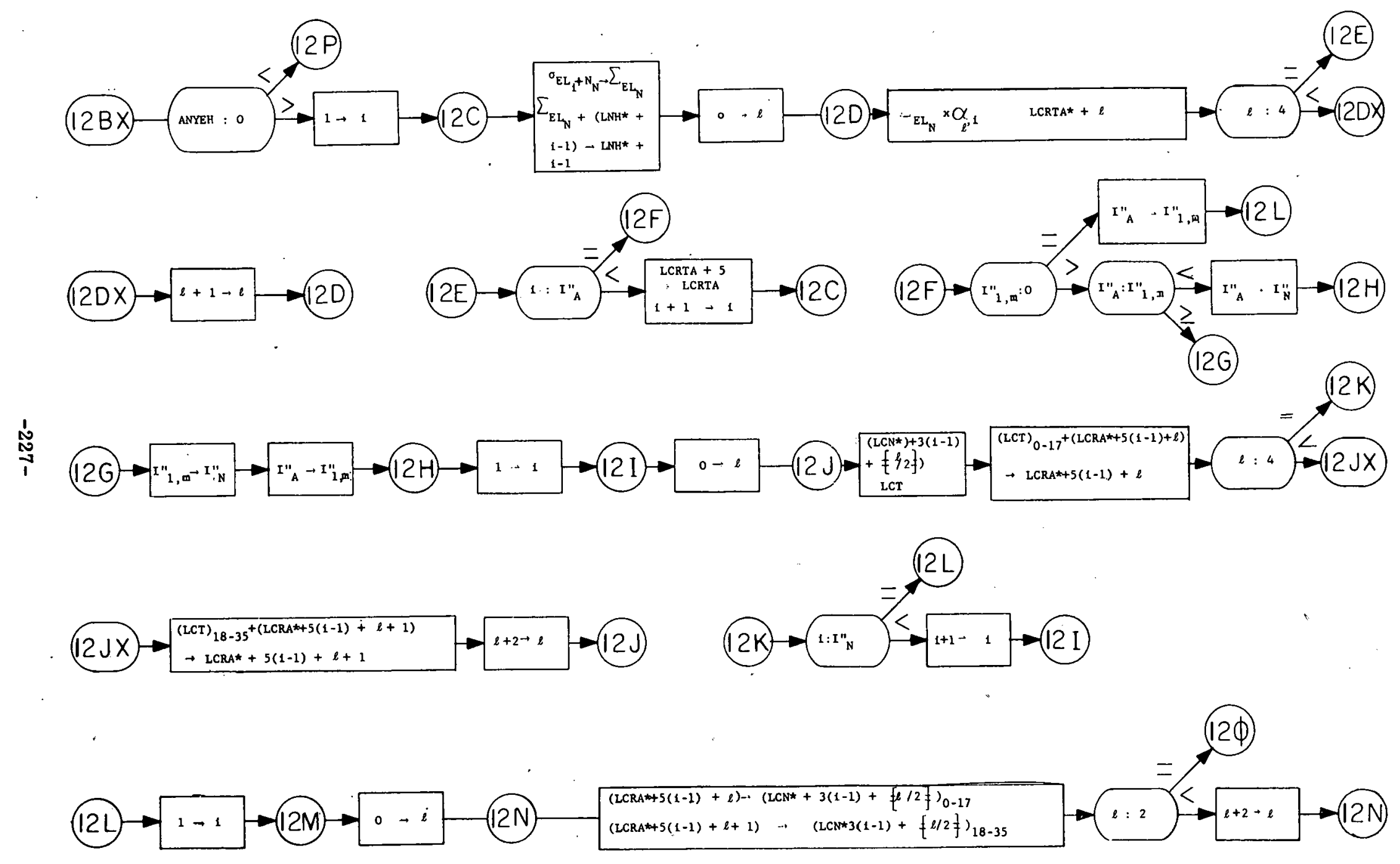




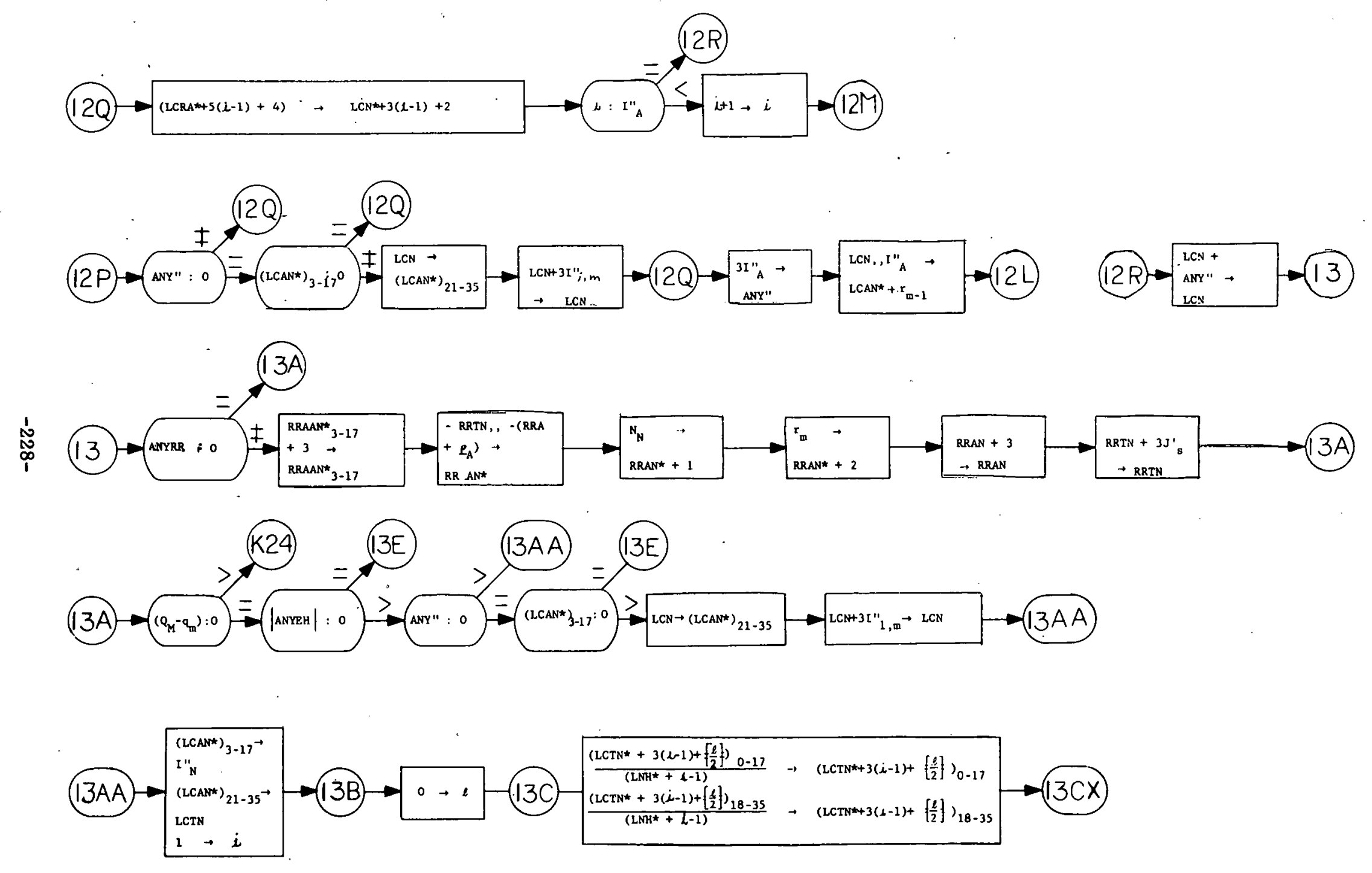

c 2 

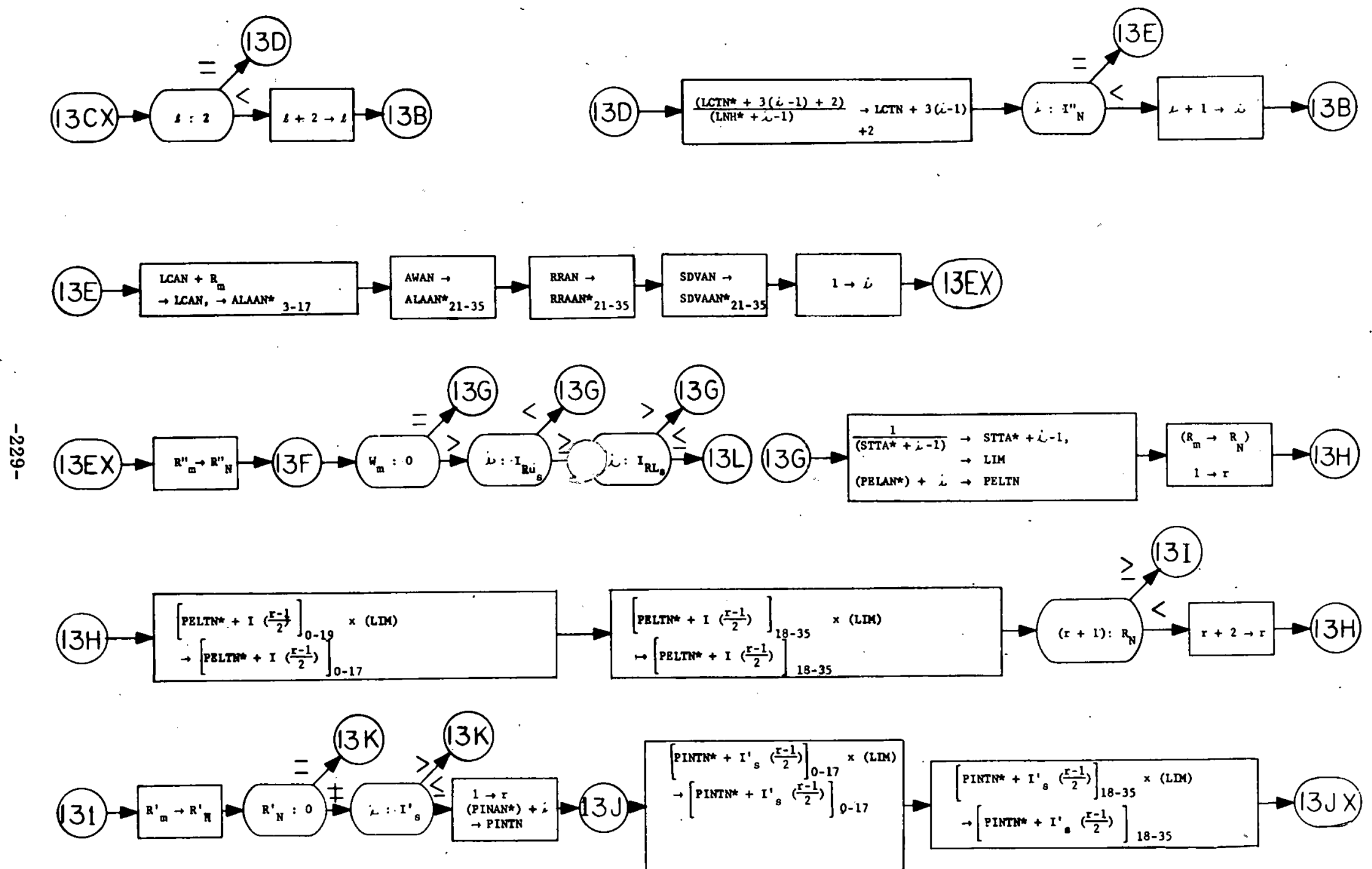


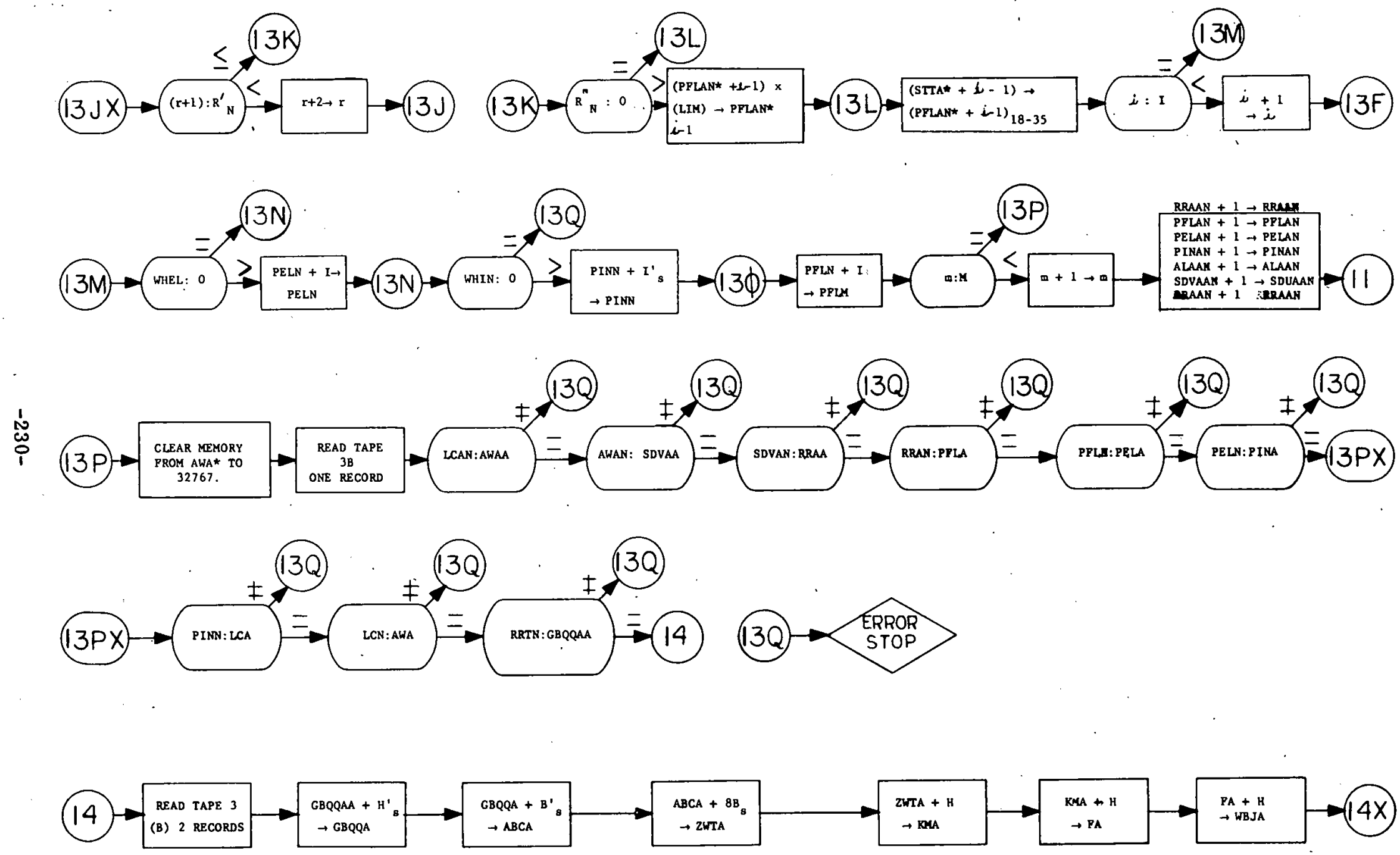




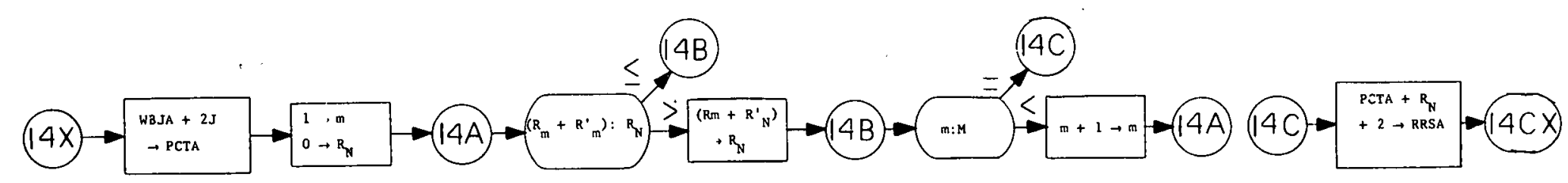

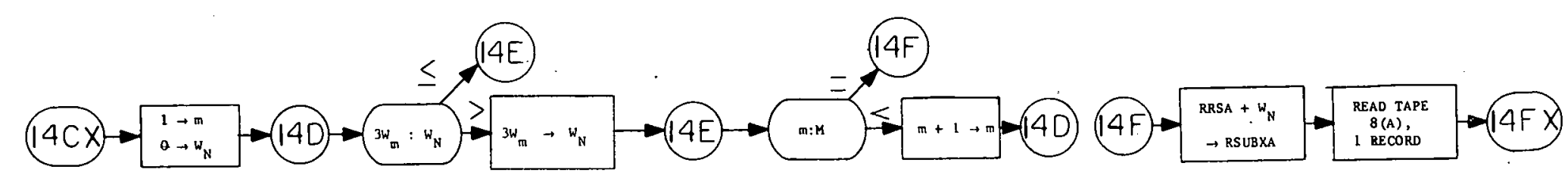
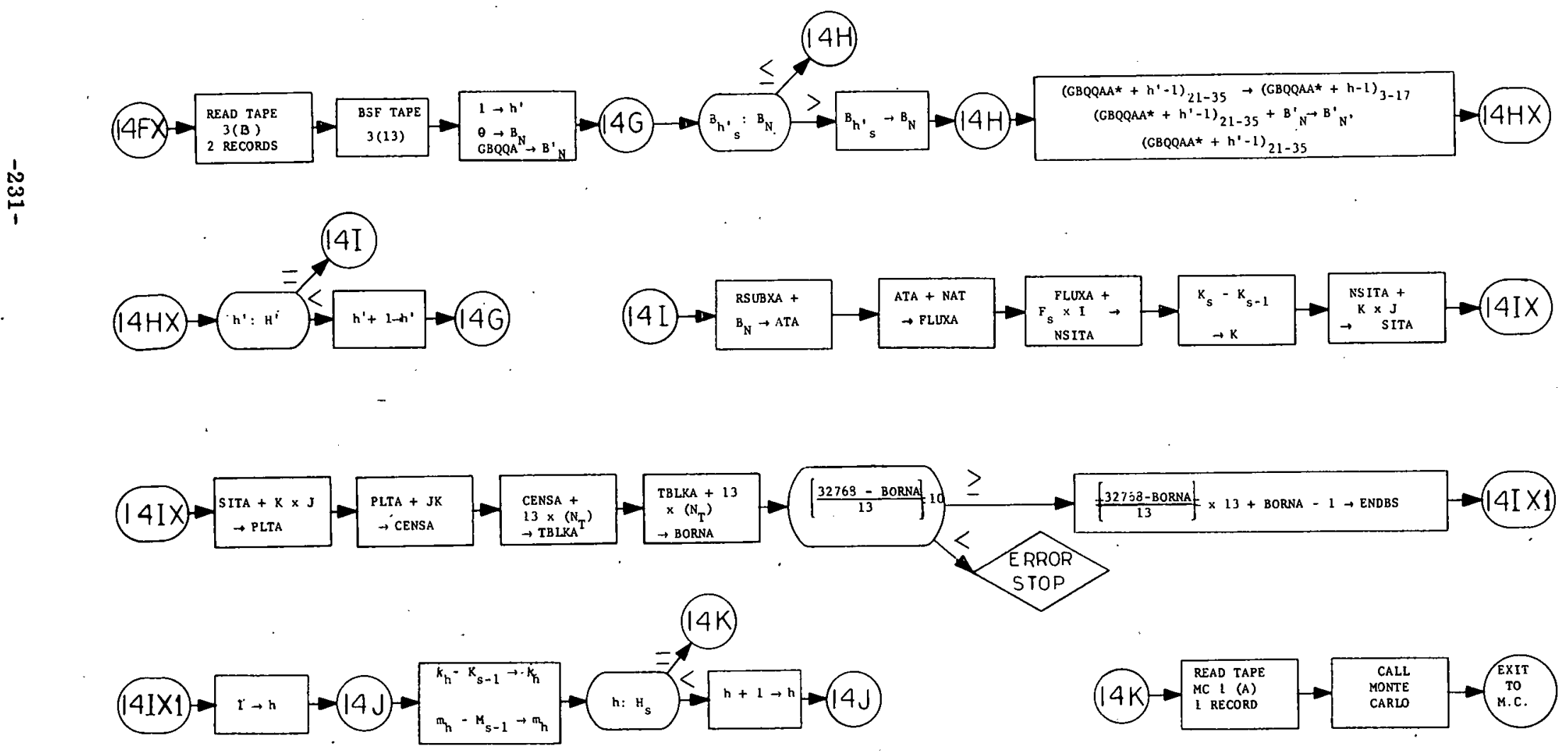
POST MONTE CARLO.

(15) $\rightarrow$ (ANY HC):0 :

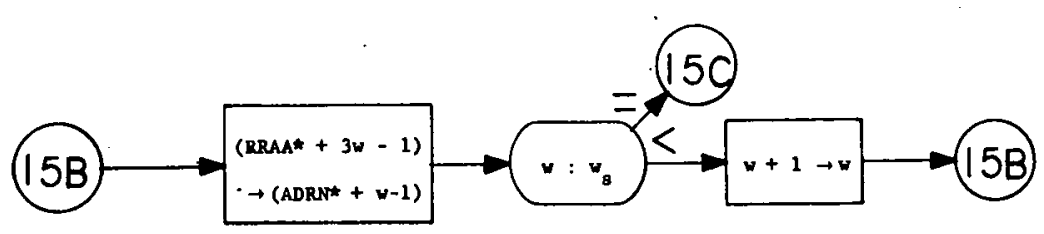

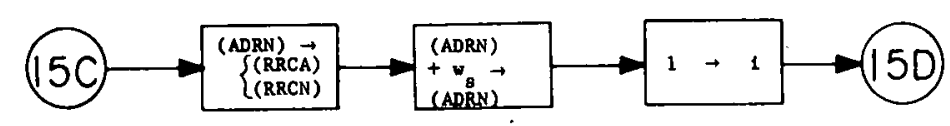

(150)
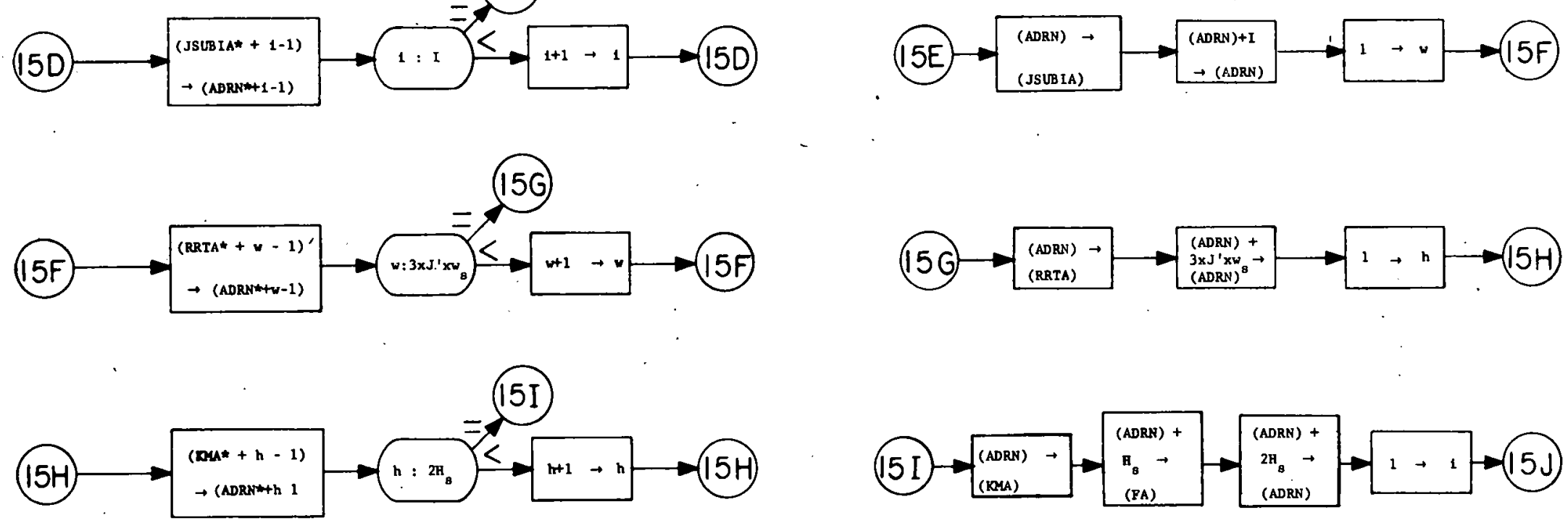

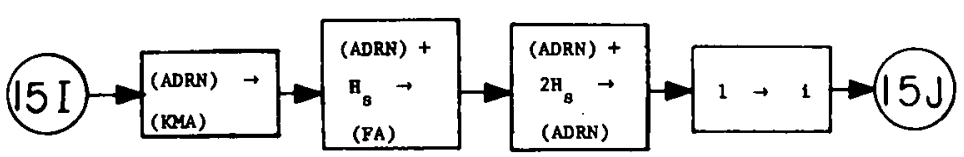



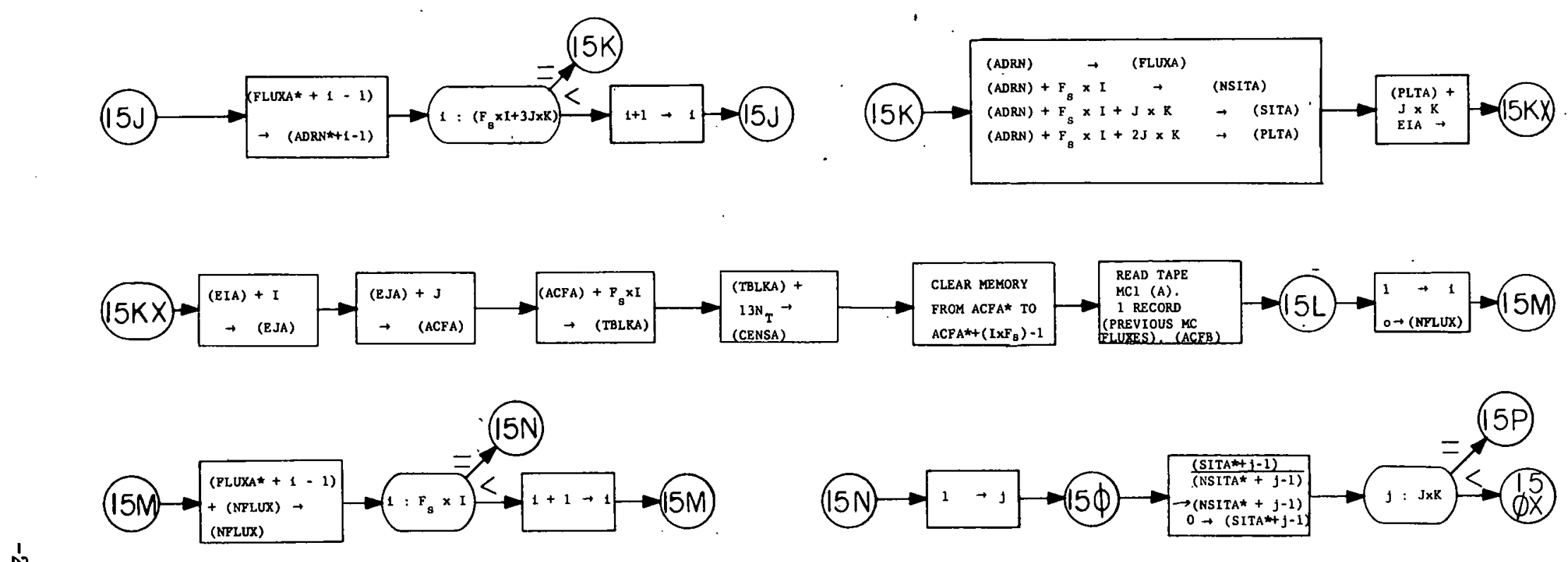

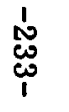

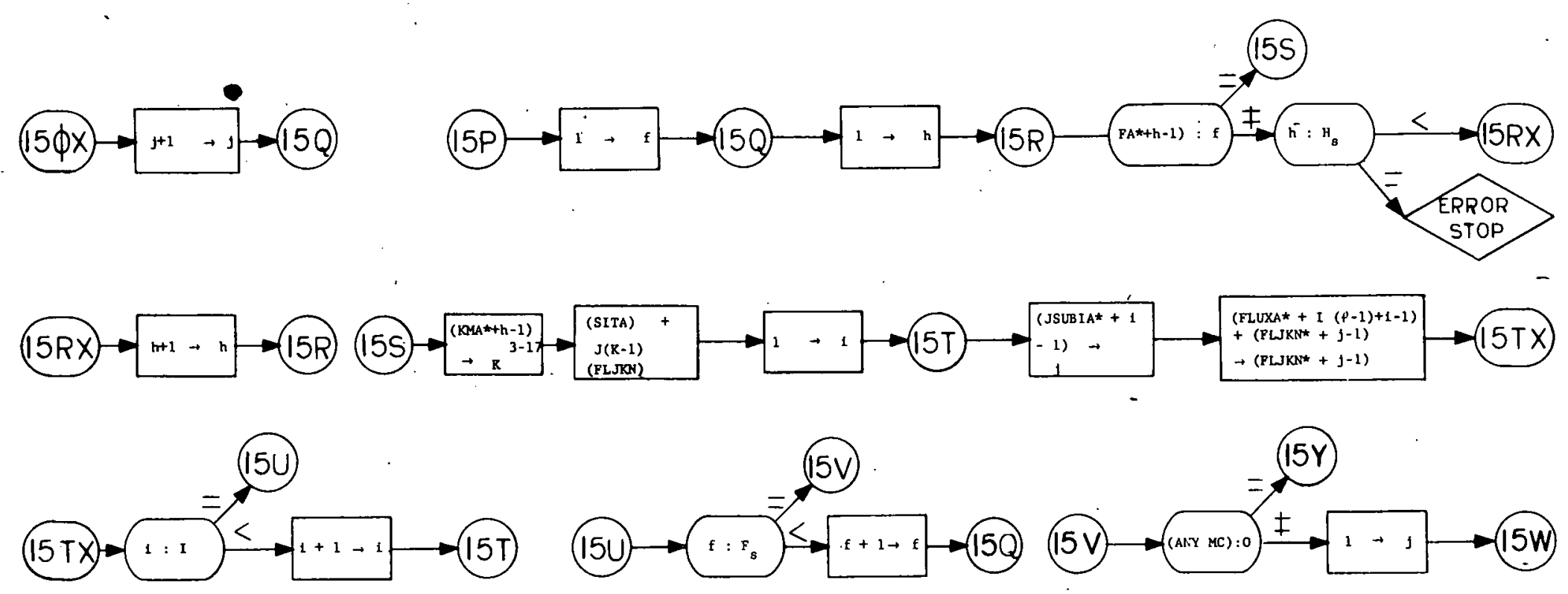




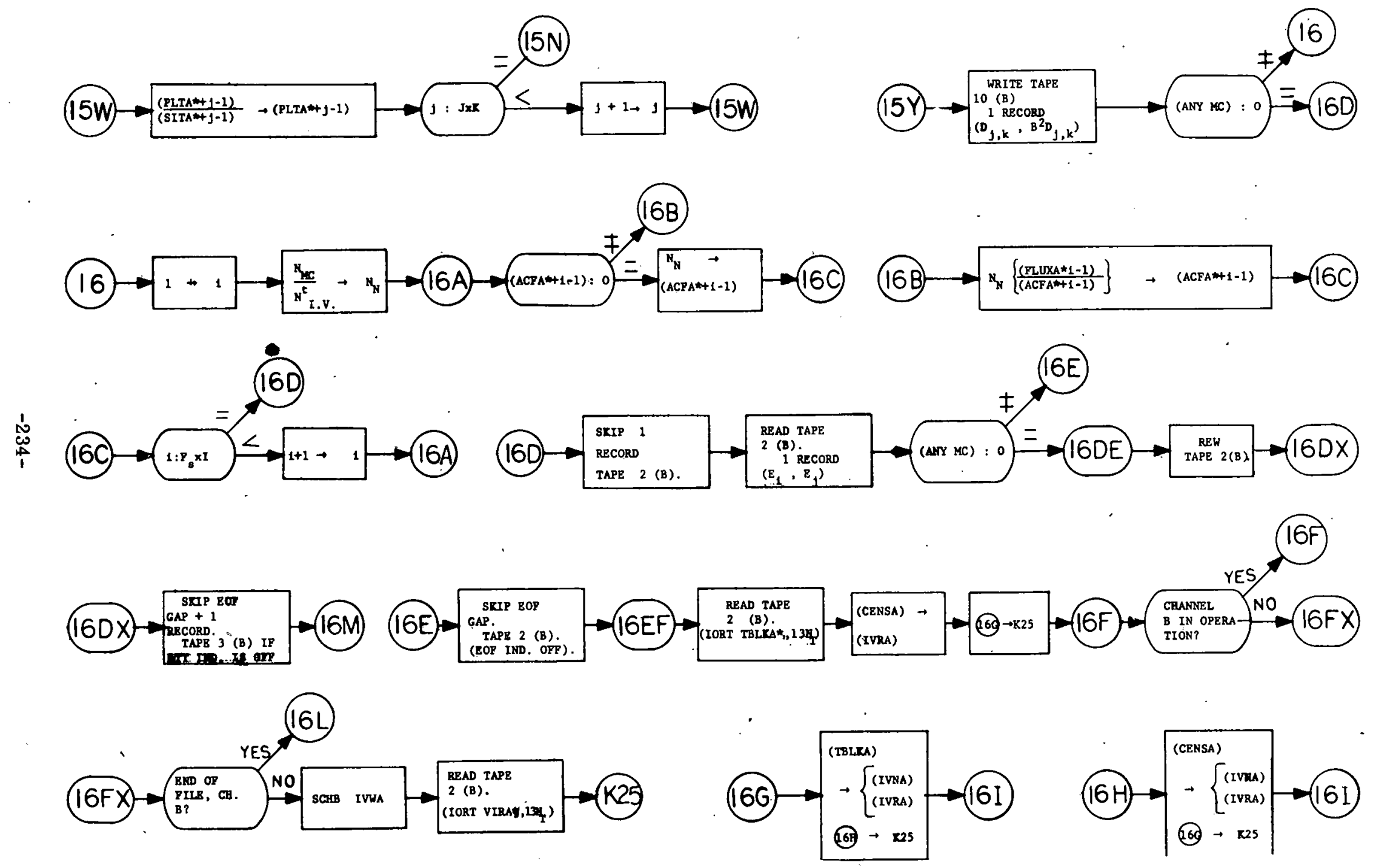




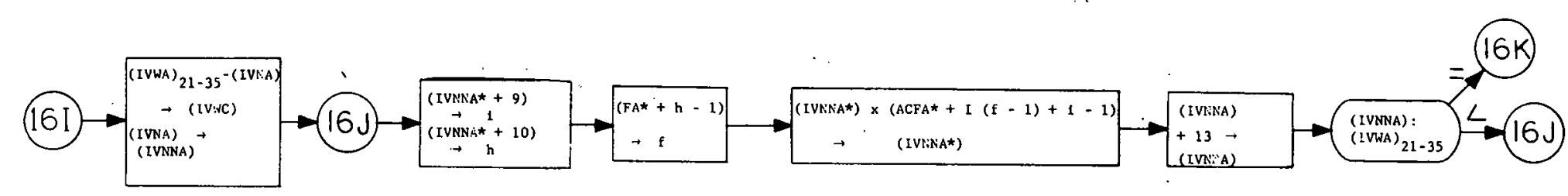
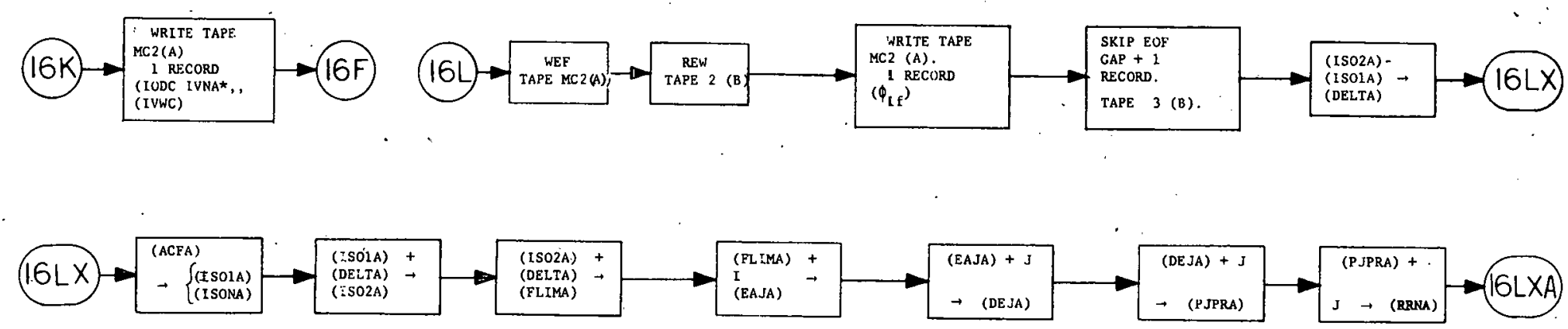

心
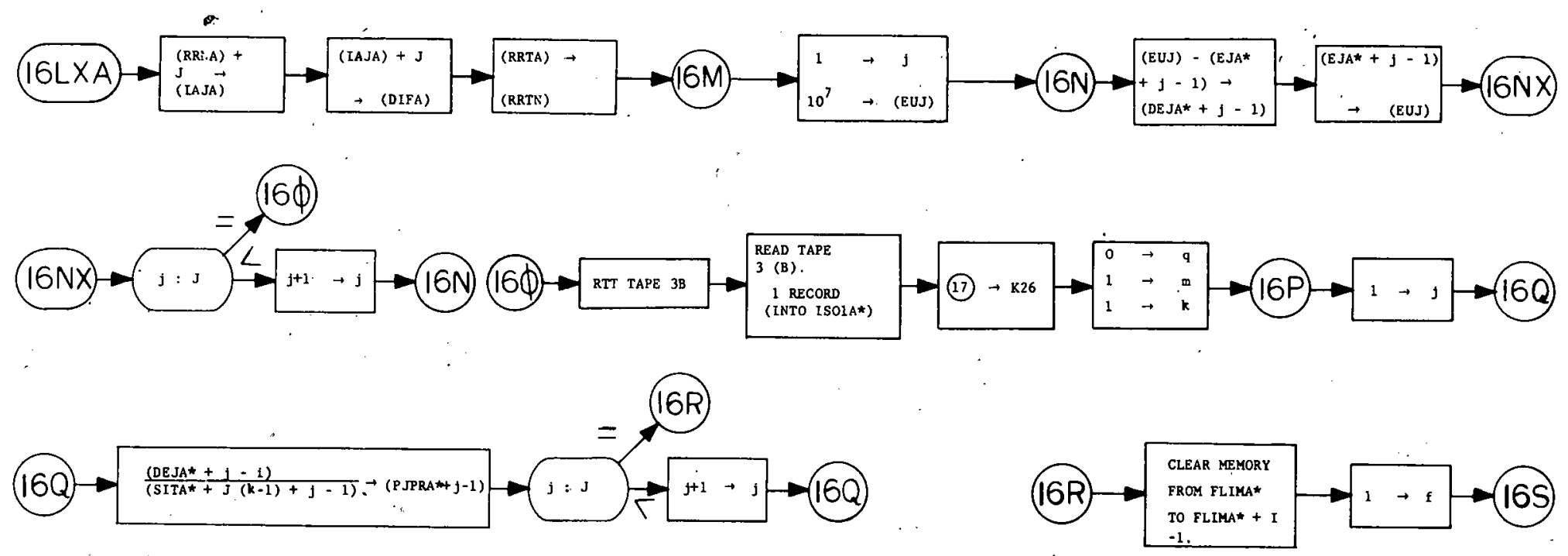


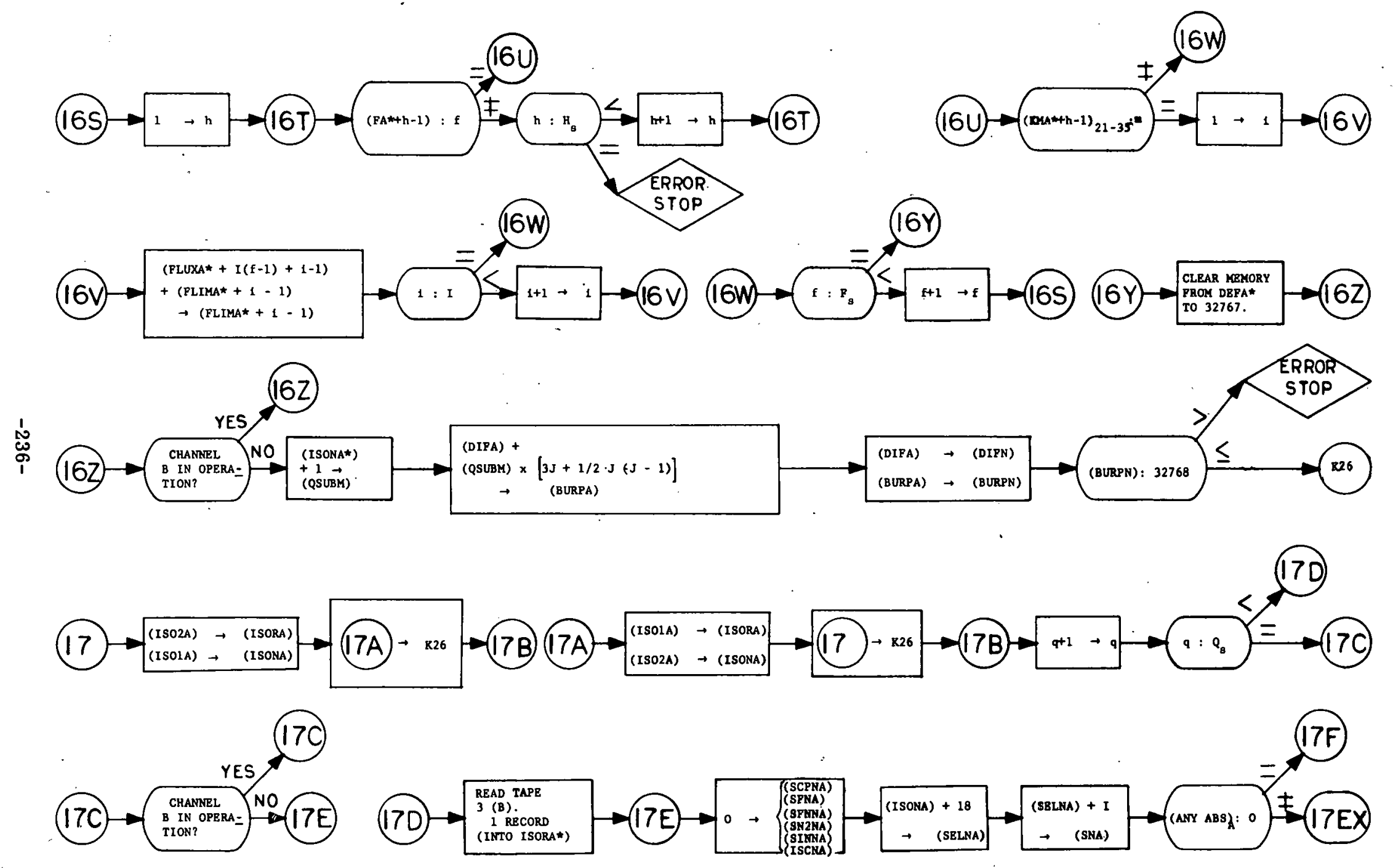




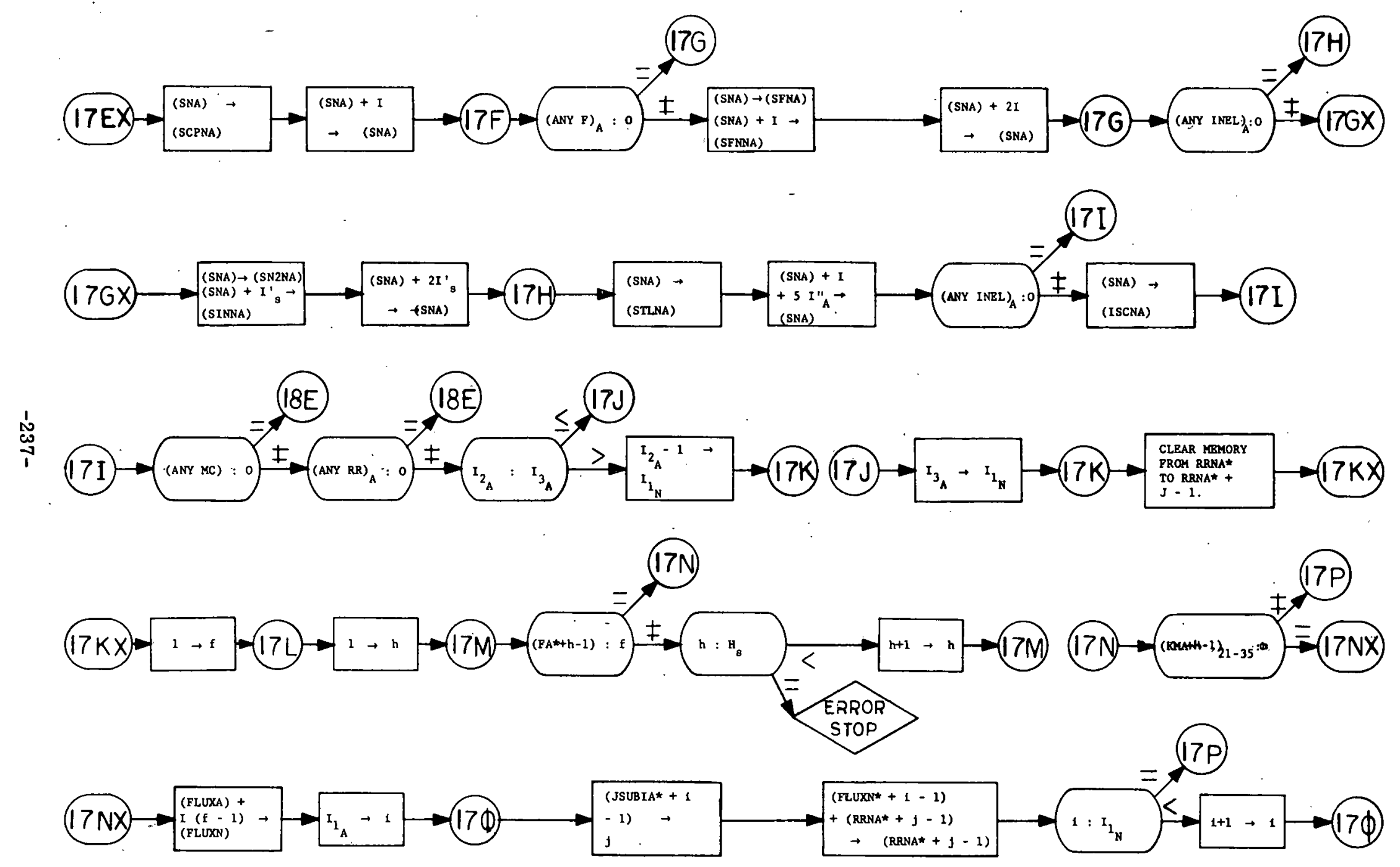



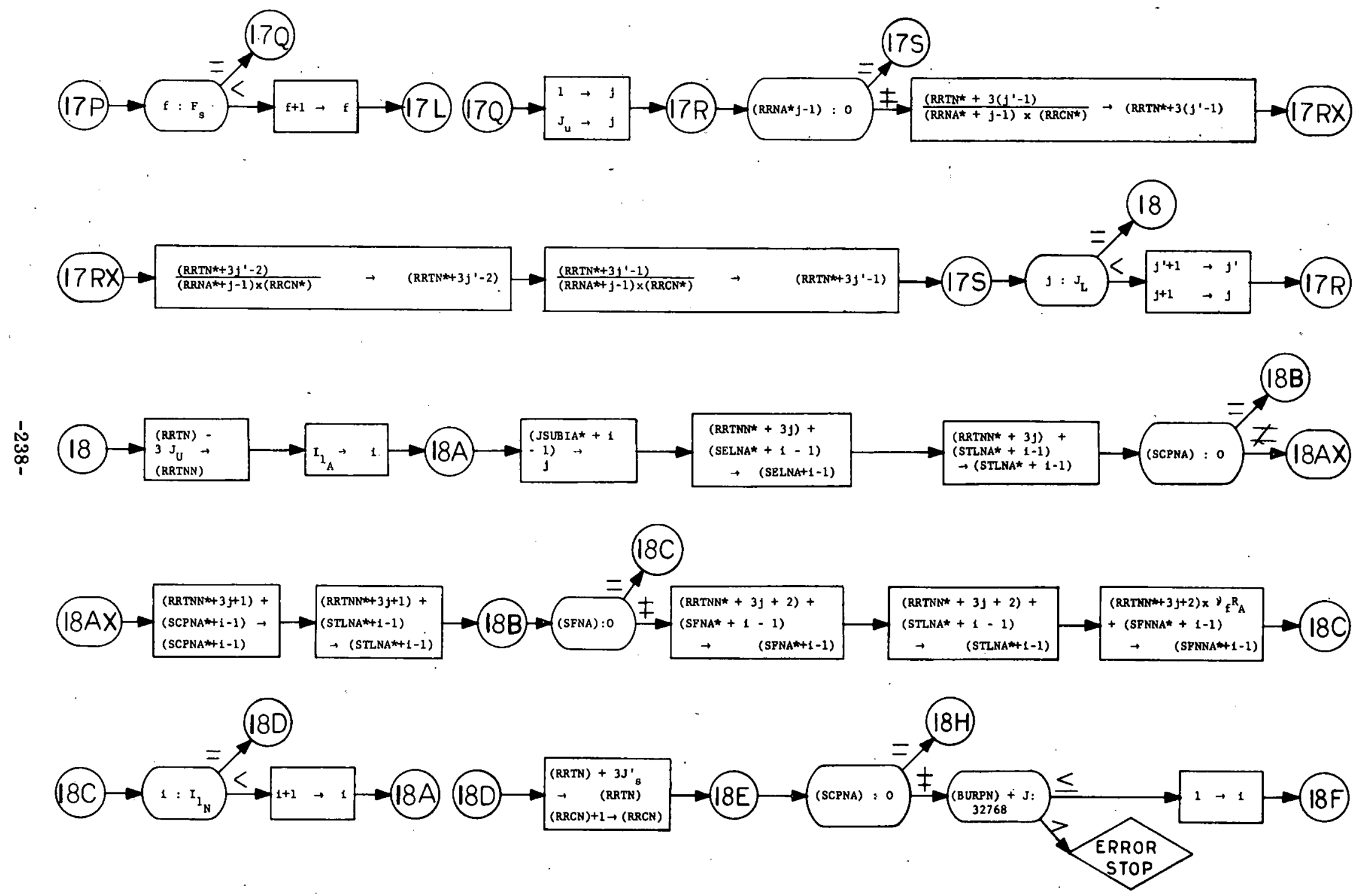


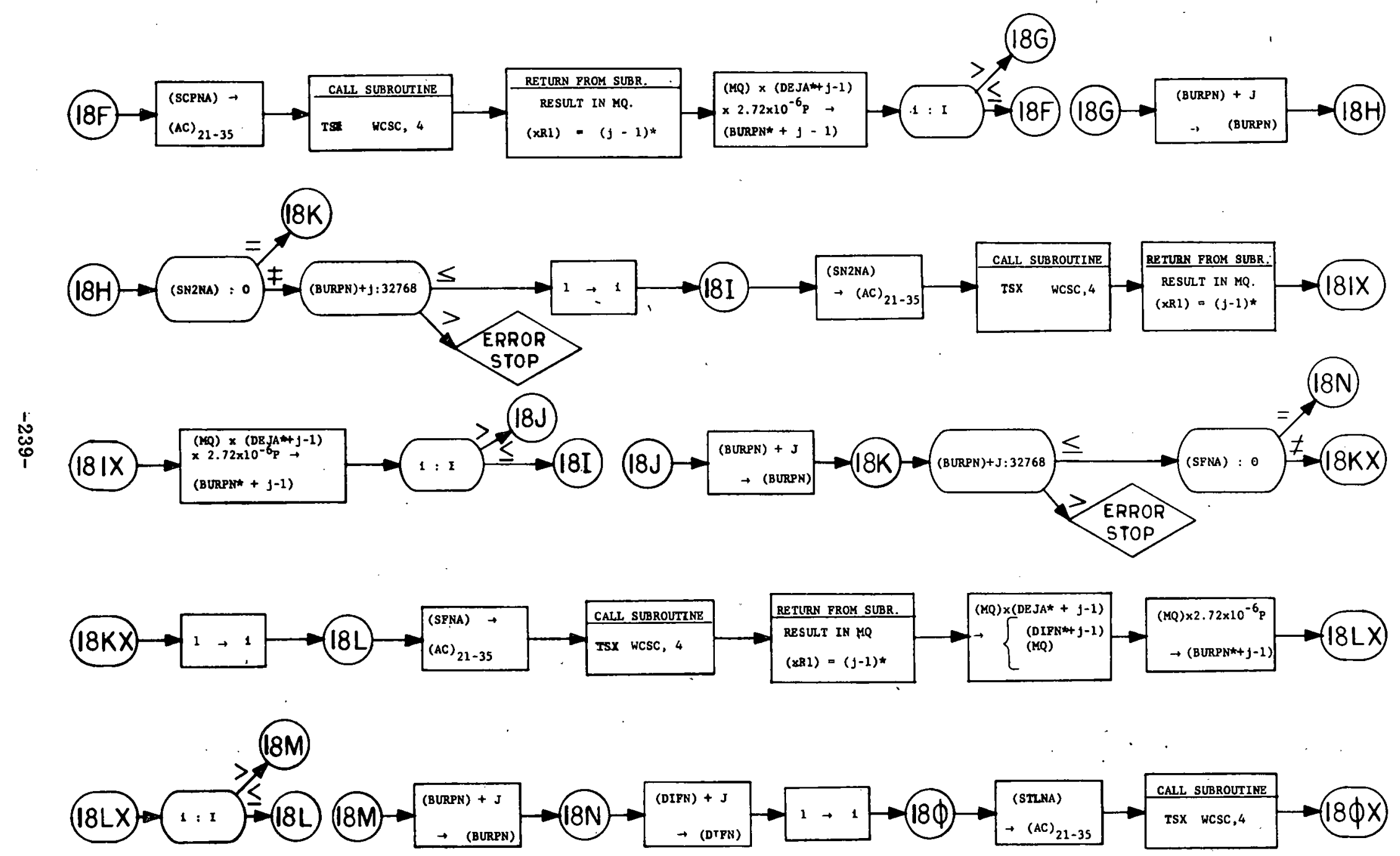




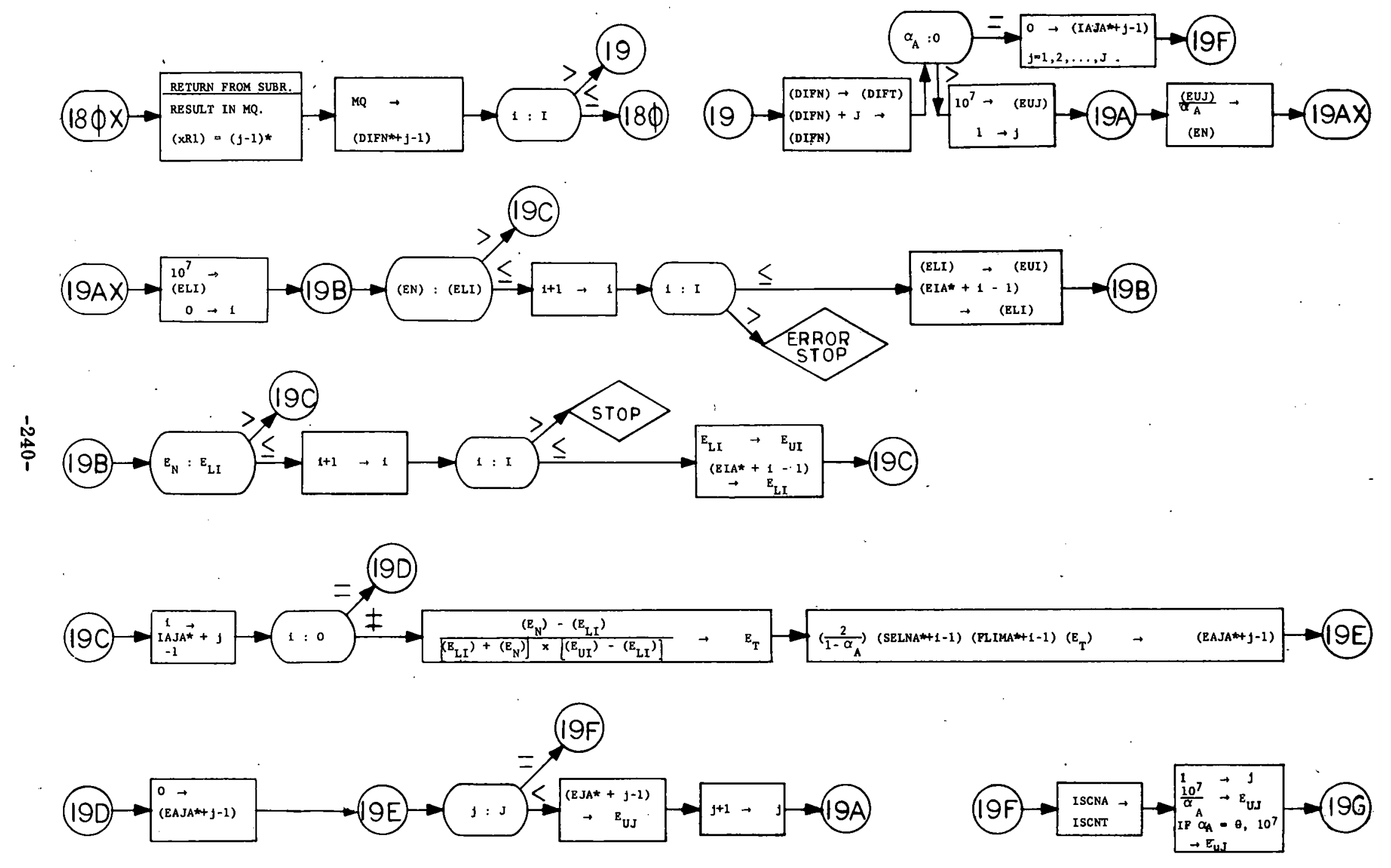

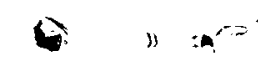

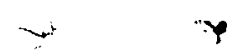

$\therefore \quad ;$ 


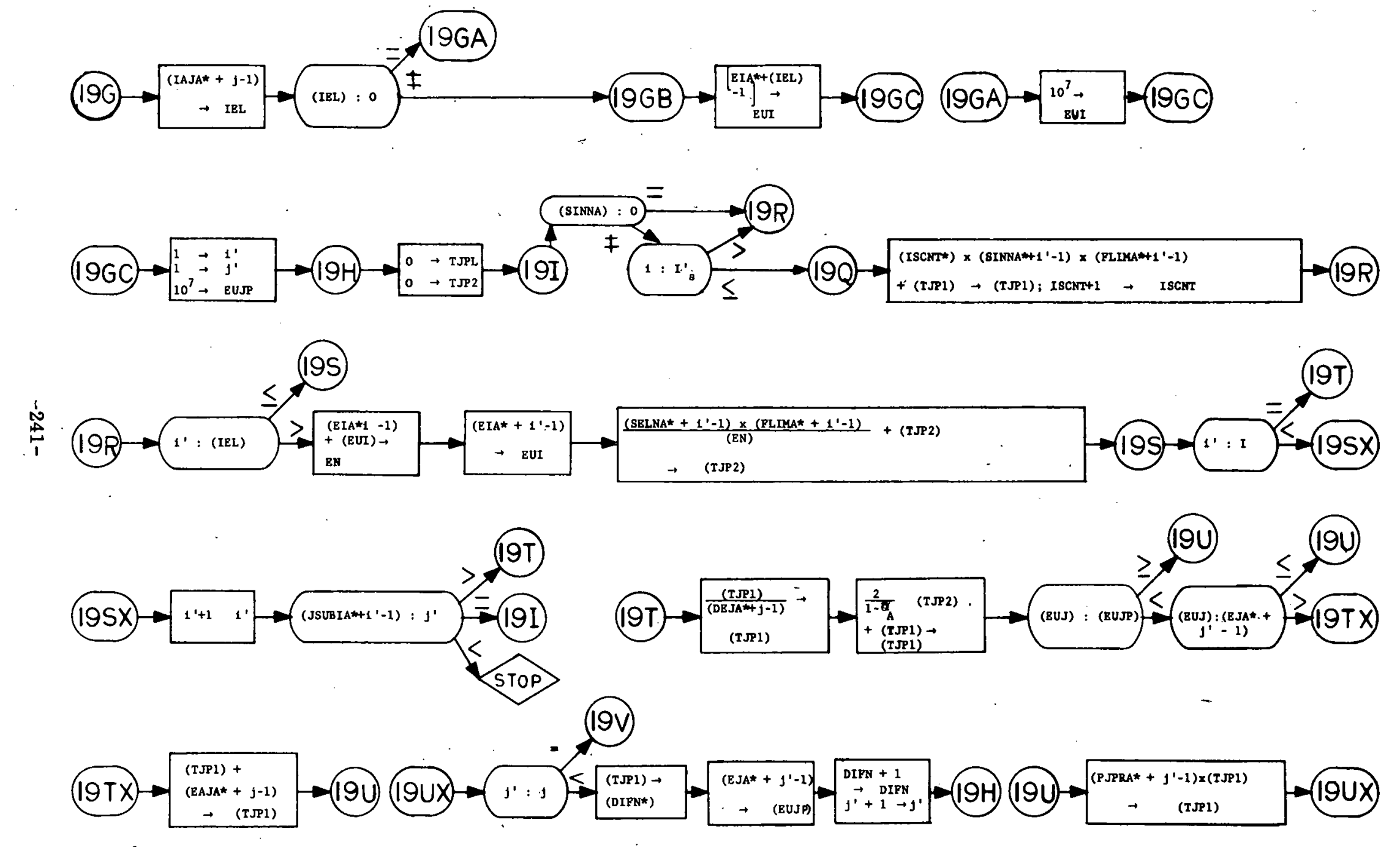




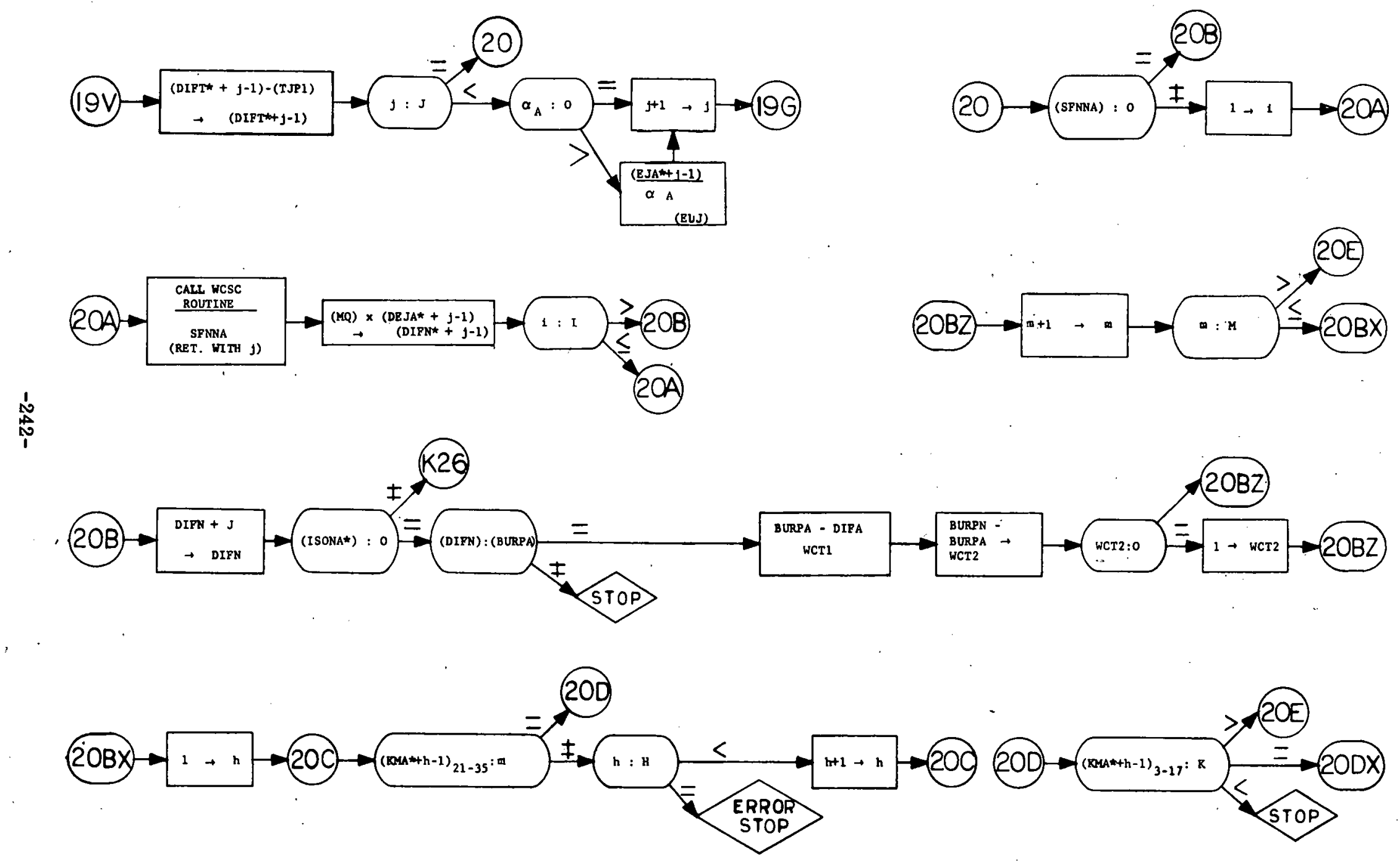

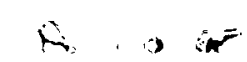



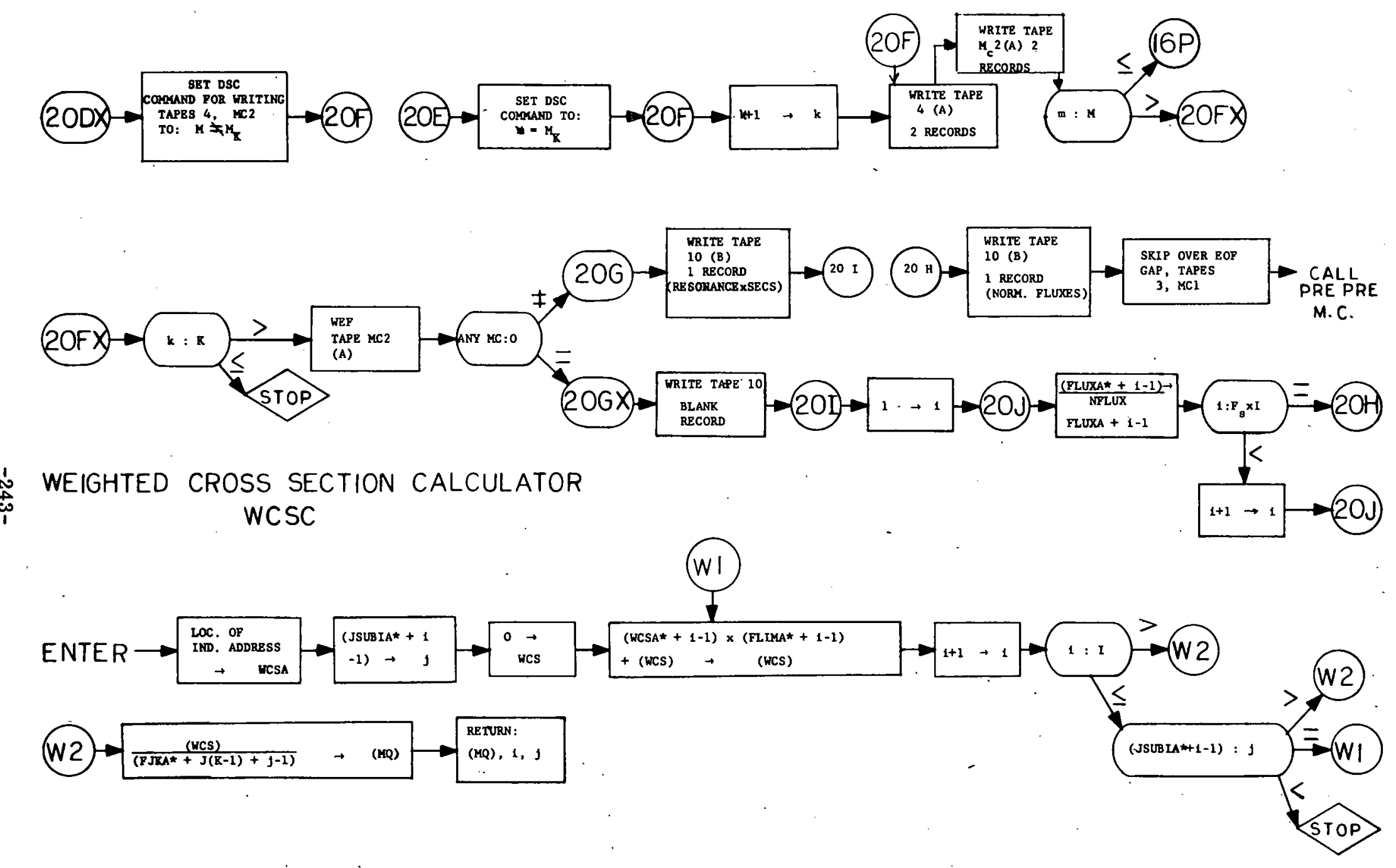

ENTER: FHA of a block in AC.
$(1-1) *$ in XR2.

RET: Weighted o in MO

$(j-1) *$ in $\times R 1$
$(1-1) *$ in $\times R 2$ 